University of Tennessee Health Science Center

UTHSC Digital Commons

\title{
$5-2011$
}

\section{A Novel Shear Thinning and Thixotropic Plga Micro Particulate Suspension System for Controlled Drug Delivery}

Sonia Bedi

University of Tennessee Health Science Center

Follow this and additional works at: https://dc.uthsc.edu/dissertations

Part of the Pharmacy and Pharmaceutical Sciences Commons

\section{Recommended Citation}

Bedi, Sonia, "A Novel Shear Thinning and Thixotropic Plga Micro Particulate Suspension System for Controlled Drug Delivery" (2011). Theses and Dissertations (ETD). Paper 23. http://dx.doi.org/10.21007/ etd.cghs.2011.0024.

This Dissertation is brought to you for free and open access by the College of Graduate Health Sciences at UTHSC Digital Commons. It has been accepted for inclusion in Theses and Dissertations (ETD) by an authorized administrator of UTHSC Digital Commons. For more information, please contact jwelch30@uthsc.edu. 


\title{
A Novel Shear Thinning and Thixotropic Plga Micro Particulate Suspension System for Controlled Drug Delivery
}

\begin{abstract}
Poly lactide-co-glycolide (PLGA) polymer has been the polymer of choice for many parenteral controlled drug release applications. This is mainly due to the inherent advantages of this polymer i.e. biodegradability, biocompatibility and non-toxic nature. The polymer, however, has a characteristic degradation pattern whereby the final erosion phase does not begin until the polymer reaches a certain molecular weight (MW) limit. After this, the accumulated acidic degradation byproducts initiate a bulk erosion phenomenon that leads to a disruption of the polymer matrix and release of the remaining drug in a short period of time. Furthermore, most of the delivery systems or devices made from PLGA polymer i.e. microspheres or polymeric solutions forming in situ implants are associated with another limitation of an initial drug burst. A major percentage of the drug is released during first few hours thereby leaving a relatively smaller portion of the total drug load to be released slowly over the remaining duration. By and large it results in a characteristic "tri-phasic release pattern" from the PLGA matrices consisting of a first burst release phase, a second plateau phase and a final burst release phase.

Plasticizer molecules are well known for their ability to create a flexible polymer matrix thereby allowing a continuous drug release. Polymer solutions made with certain highly hydrophilic plasticizers however, are not completely devoid of the initial burst release of drug due to a lag time between injection of the polymer solution and complete precipitation of the polymer. Other most common drawbacks of the PLGA polymeric solution based delivery systems is their high viscosity and therefore painful injections and poor injectability, and a variable surface area of implant resulting in a highly variable drug release.
\end{abstract}

The objective of present work was therefore to make an in situ polymer gelling system formulated as polymer micro particle suspension for low viscosity and better injectability. The system comprised of a hydrophobic polymer plasticizer(s) that resulted in a more diffusion controlled drug release. Necessary physical stability of the suspension based formulation was derived from a glycerol lipid (polymer immiscible component) and polycaprolactone (PCL) crystallites/spherulites. The system was structurally characterized by differential scanning calorimetry (DSC), scanning electron microscopy (SEM) and polarized microscopy studies. Drug release mechanism was studied by conducting plasticizer release studies and mathematical modeling.

Results indicated transformation of drug release from an erosion based process to a complete diffusion controlled phenomenon by the use of a hydrophobic polymer plasticizer(s). Glycero-lipid provided extra physical stability to the PLGA micro particle suspension. The PCL crystallites not only provided the essential stability characteristics to the delivery system i.e. structural stability of the micro particle suspension and a thixotropic and shear thinning behavior for the ease of injectability but further controlled the initial drug release. Biocompatibility studies indicated that the in situ PLGA micro particulate implant formulation containing PCL crystallites is safe and biocompatible with only a normal tissue response.

\section{Document Type}

Dissertation

Degree Name

Doctor of Philosophy (PhD)

\section{Program}

Pharmaceutical Sciences 


\section{Research Advisor}

James R. Johnson, Ph.D.

\section{Keywords}

Controlled release, PCL crystallites, plasticizer, PLGA micro particles, shear thinning, thixotropic.

\section{Subject Categories}

Medicine and Health Sciences | Pharmacy and Pharmaceutical Sciences 


\title{
A NOVEL SHEAR THINNING AND THIXOTROPIC PLGA MICROPARTICULATE SUSPENSION SYSTEM FOR CONTROLLED DRUG DELIVERY
}

\author{
A Dissertation \\ Presented for \\ The Graduate Studies Council \\ The University of Tennessee \\ Health Science Center
}

\author{
In Partial Fulfillment \\ Of the Requirements for the Degree \\ Doctor of Philosophy \\ From The University of Tennessee
}

By

Sonia Bedi

May 2011 
Copyright (C) 2011 by Sonia Bedi. All rights reserved. 


\section{DEDICATION}

I dedicate this work to my parents

Mrs. Ravinder Kaur and Mr. Rajinder Singh Bedi, for their love and support. 


\section{ACKNOWLEDGEMENTS}

The printed pages of this dissertation hold far more than the culmination of years of study. These pages also reflect the relationships with many generous and inspiring people I have met since beginning my graduate work. The list is long, but I cherish each contribution to my development as a scholar.

I am highly indebted and heartily thankful to my first major advisor, Dr. Atul J. Shukla, who inspired me to think rationally and scientifically. His encouragement, supervision and support throughout my Ph.D. years enabled me to develop an understanding of the subject. He was a gracious mentor who demonstrated that rigorous scholarship can and must be accessible to everyone, that social change is central to intellectual work and, as such, scholars have a responsibility to use the privileges of academia to imagine and create a better world. I would extend special thanks and acknowledgement to Dr. James R. Johnson who agreed to mentor me and offer his guidance and support during the last year of my Ph.D. work after the sad demise of Dr. Shukla. I would also like to thank my committee members, Dr. George C. Wood, Dr. Bernd Meibohm, Dr. James T. Dalton and Dr. Vivian Loveless for their invaluable guidance and suggestions in my work at the university. I further would like to recognize the irreplaceable continuous love and support that I got from my lab members and also thank my previous lab member, Dr. Gerald Rajan for his input on the regression analysis.

Most of all, I would like to thank my parents for being supportive, forgiving, generous and loving without whom I could not have survived the long haul, my brothers, Mandeep and Amandeep for their continuous encouragement, motivation and belief in me.

The acknowledgment would certainly not be complete without mentioning my husband Himanshu. He has always been an enormous support and source of motivation for me inside and outside my work environment. He helped me in getting out of the loop of puzzlement many times during my Ph.D. by providing his practical inputs.

I would also like to thank Stacey Barnett, Ernestine, Dr Jackson Scott and Dr Tim Mandrell for providing me all the possible practical help and guidance for the animal study. I sincerely thank Jin Xu who genuinely helped me while working with the rats and deserves special appreciation.

Special acknowledgement goes to Dr. Stanley Kosanke who helped me with the histology studies of the rat tissue specimens and provided me with a good understanding of the subject.

Finally, I would like to thank Ms. Shirley Hancock for her help and suggestions during my dissertation formatting and University of Tennessee for providing me this wonderful opportunity for my graduate studies in presence of all the wonderful people. 


\begin{abstract}
Poly lactide-co-glycolide (PLGA) polymer has been the polymer of choice for many parenteral controlled drug release applications. This is mainly due to the inherent advantages of this polymer i.e. biodegradability, biocompatibility and non-toxic nature. The polymer, however, has a characteristic degradation pattern whereby the final erosion phase does not begin until the polymer reaches a certain molecular weight (MW) limit. After this, the accumulated acidic degradation byproducts initiate a bulk erosion phenomenon that leads to a disruption of the polymer matrix and release of the remaining drug in a short period of time. Furthermore, most of the delivery systems or devices made from PLGA polymer i.e. microspheres or polymeric solutions forming in situ implants are associated with another limitation of an initial drug burst. A major percentage of the drug is released during first few hours thereby leaving a relatively smaller portion of the total drug load to be released slowly over the remaining duration. By and large it results in a characteristic "tri-phasic release pattern" from the PLGA matrices consisting of a first burst release phase, a second plateau phase and a final burst release phase.
\end{abstract}

Plasticizer molecules are well known for their ability to create a flexible polymer matrix thereby allowing a continuous drug release. Polymer solutions made with certain highly hydrophilic plasticizers however, are not completely devoid of the initial burst release of drug due to a lag time between injection of the polymer solution and complete precipitation of the polymer. Other most common drawbacks of the PLGA polymeric solution based delivery systems is their high viscosity and therefore painful injections and poor injectability, and a variable surface area of implant resulting in a highly variable drug release.

The objective of present work was therefore to make an in situ polymer gelling system formulated as polymer micro particle suspension for low viscosity and better injectability. The system comprised of a hydrophobic polymer plasticizer(s) that resulted in a more diffusion controlled drug release. Necessary physical stability of the suspension based formulation was derived from a glycerol lipid (polymer immiscible component) and polycaprolactone (PCL) crystallites/spherulites. The system was structurally characterized by differential scanning calorimetry (DSC), scanning electron microscopy (SEM) and polarized microscopy studies. Drug release mechanism was studied by conducting plasticizer release studies and mathematical modeling.

Results indicated transformation of drug release from an erosion based process to a complete diffusion controlled phenomenon by the use of a hydrophobic polymer plasticizer(s). Glycero-lipid provided extra physical stability to the PLGA micro particle suspension. The PCL crystallites not only provided the essential stability characteristics to the delivery system i.e. structural stability of the micro particle suspension and a thixotropic and shear thinning behavior for the ease of injectability but further controlled the initial drug release. Biocompatibility studies indicated that the in situ PLGA micro particulate implant formulation containing PCL crystallites is safe and biocompatible with only a normal tissue response. 


\section{TABLE OF CONTENTS}

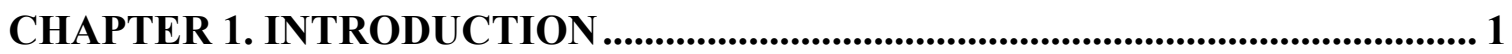

1.1. Biodegradable Injectable In Situ Forming Drug Delivery Systems ........................

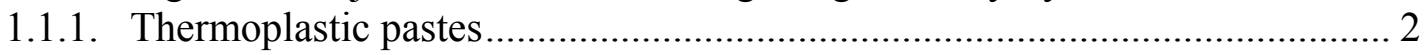

1.1.2. In situ cross-linked polymer systems ......................................................... 2

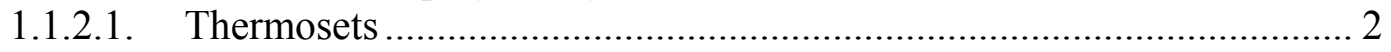

1.1.2.2. Photo cross-linked gels ................................................................. 3

1.1.2.3. Ion mediated gelation ...................................................................... 3

1.1.3. In situ polymer precipitation ................................................................... 3

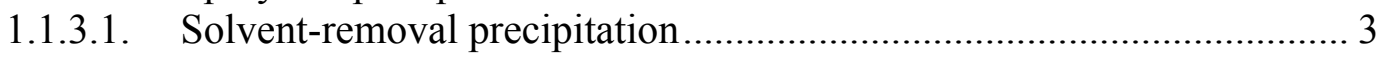

1.1.3.2. Thermally induced sol-gel transitions................................................ 5

1.1.4. In situ solidifying organogels................................................................. 5

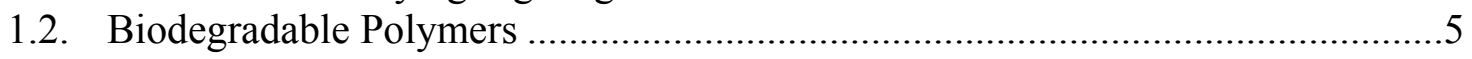

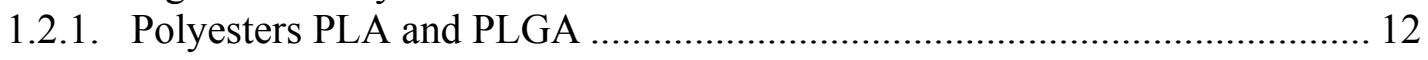

1.2.1.1. Biodegradation of PLGA polymer ..................................................... 12

1.2.1.2. Biocompatibility of PLGA polymer.................................................. 14

1.2.1.3. Physicochemical properties of PLGA ................................................. 14

1.2.1.3.1. Molecular weight and polydispersity ............................................. 14

1.2.1.3.2. Optical activity and crystallinity .................................................... 15

1.2.1.3.3. Glass transition temperature $(\mathrm{Tg})$.................................................. 15

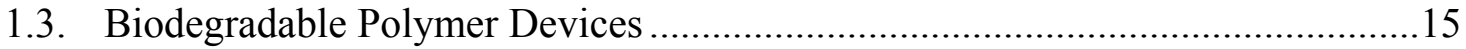

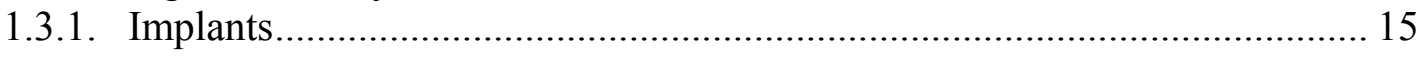

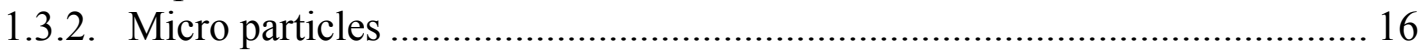

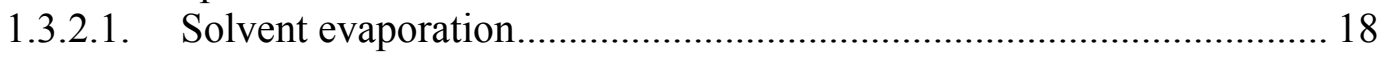

1.3.2.1.1. Conventional O/W encapsulation ................................................. 18

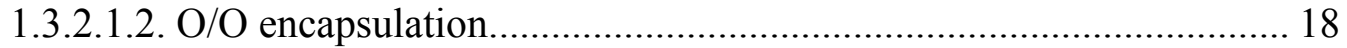

1.3.2.1.3. W/O/W multiple emulsion method................................................... 18

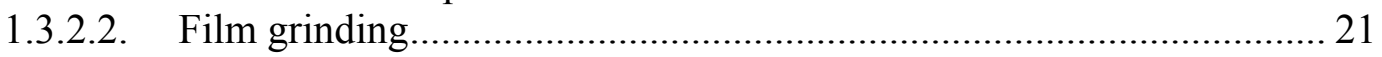

1.4. Limitations with Biodegradable Drug Delivery Devices: Burst Effect and

Tri-Phasic Release Pattern .........................................................................21

1.5. In Situ Implants Using Polymer Micro Particles....................................................24

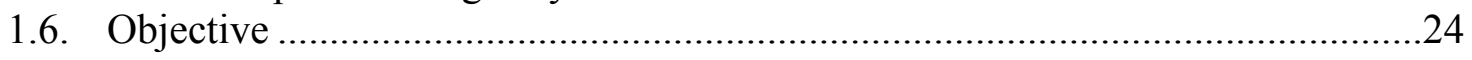

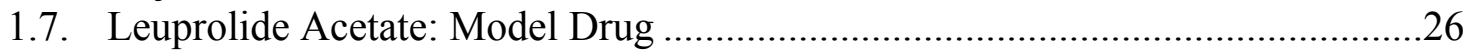

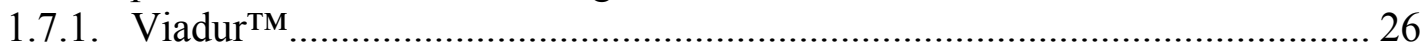

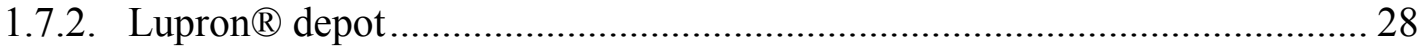

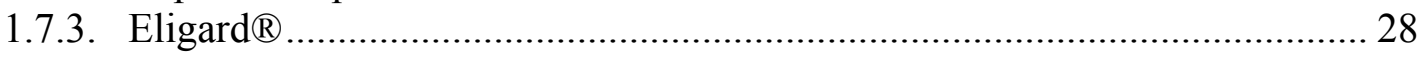

\section{CHAPTER 2. SELECTION OF DISSOLUTION METHODOLOGY ..................... 29}

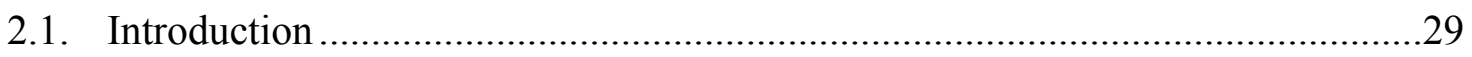

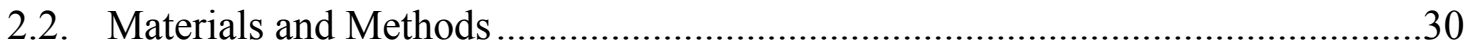

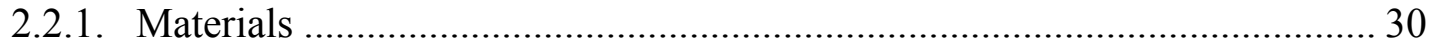

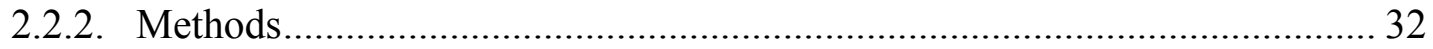

2.2.2.1. Analytical method development ....................................................... 32

2.2.2.2. Preparation of micro particles ............................................................ 32 


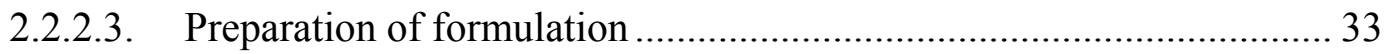

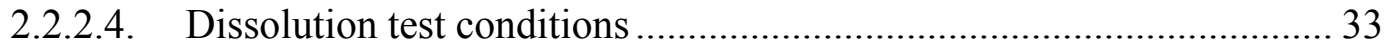

2.2.2.5. IVIVC development for Eligard ${ }^{\circledR}$ formulation................................... 33

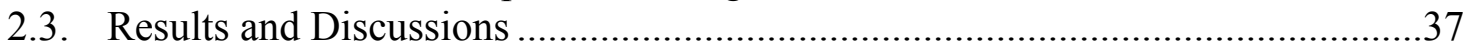

2.3.1. Analytical method development ................................................................. 37

2.3.2. Drug release studies: in vitro release set ups .............................................. 37

2.3.3. Drug release studies: marketed formulation (Eligard $\AA$ ) ................................ 43

2.3.4. Drug release studies: in house preliminary formulations ............................ 43

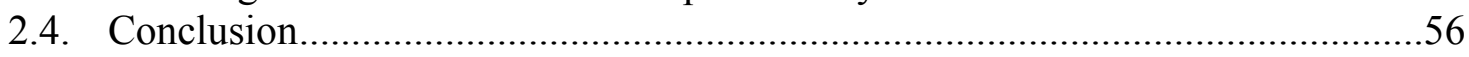

\section{CHAPTER 3. PREFORMULATION AND PRELIMINARY SCREENING STUDIES FOR FORMULATION DEVELOPMENT............................................... 57}

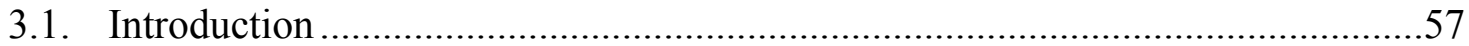

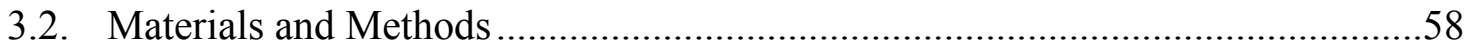

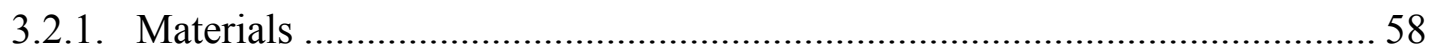

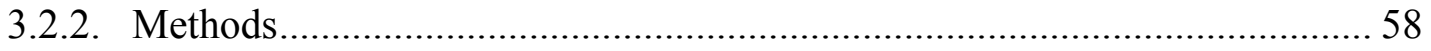

3.2.2.1. Analytical method development ......................................................... 58

3.2.2.2. Micro particle preparation and preformulation studies ......................... 60

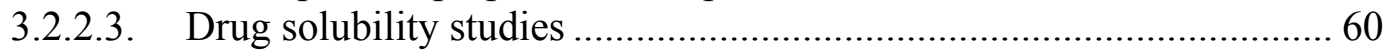

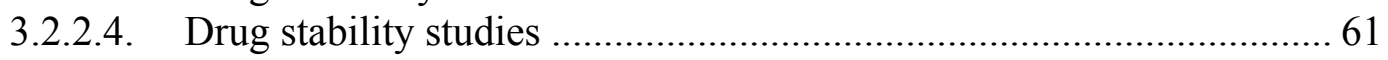

3.2.2.4.1. Drug stability in phosphate buffer saline (PBS), pH 7.4 …............... 61

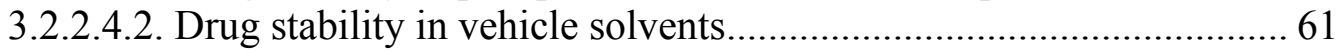

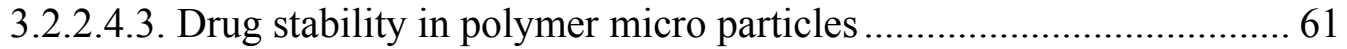

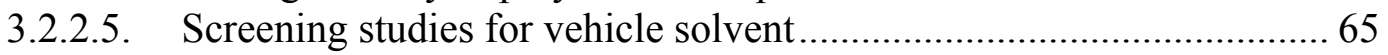

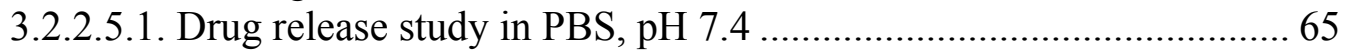

3.2.2.5.2. DSC studies for determination of glass transition temperature

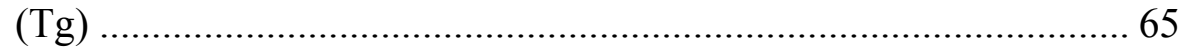

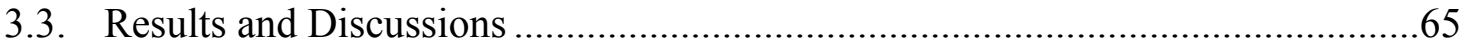

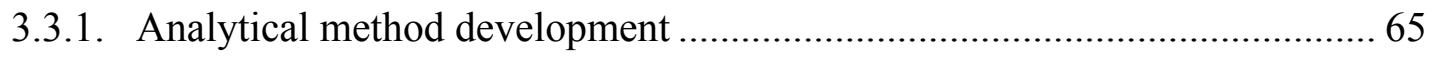

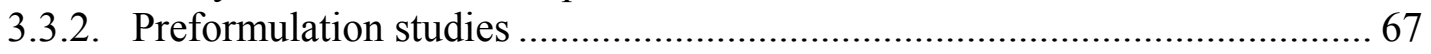

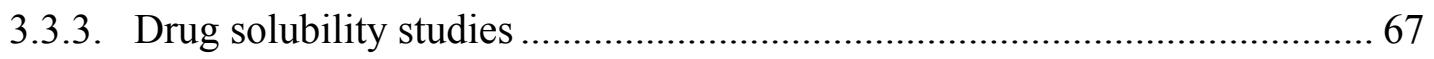

3.3.4. Drug stability studies ........................................................................... 75

3.3.5. Screening studies for vehicle solvents ..................................................... 75

3.3.6. Selection of solvents/plasticizers for formulation development ................... 86

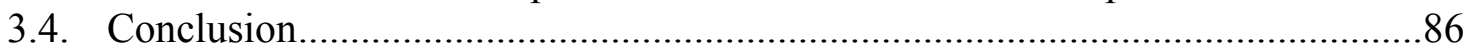

\section{CHAPTER 4. DEVELOPMENT AND CHARACTERIZATION OF AN} IN SITU GELLING PLGA MICRO PARTICLE BASED IMPLANT ...................... 90

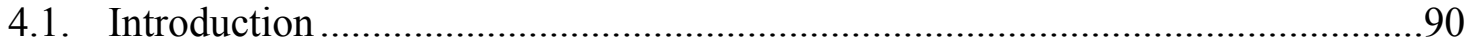

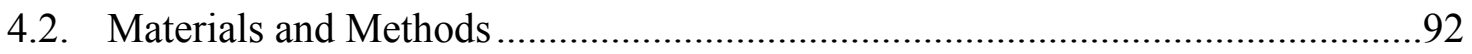

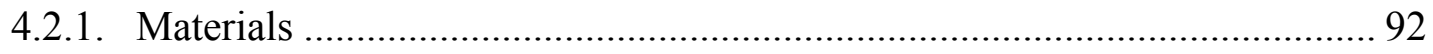

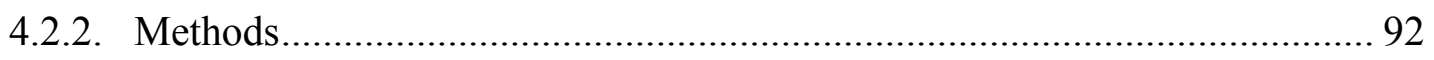

4.2.2.1. Preparation of micro particles by film grinding method...................... 92

4.2.2.2. Preparation of microspheres by modified $\mathrm{W} / \mathrm{O} / \mathrm{W}$ multiple emulsion

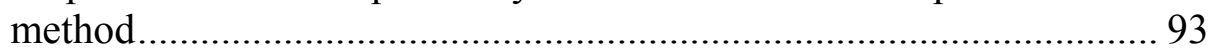

4.2.2.3. Preparation of PLGA solution formulation........................................... 93 
4.2.2.4. Assay of drug in PLGA micro particles/microspheres

4.2.2.5. Construction of phase diagrams for the binary/ternary solvent based system.

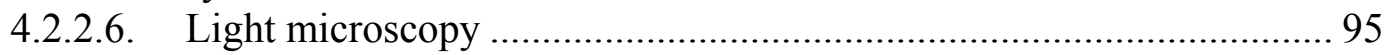

4.2.2.7. Polarized light microscopy .................................................................. 95

4.2.2.8. MDSC (Modulated differential scanning calorimetry) studies............ 95

4.2.2.9. In vitro drug release studies ............................................................... 95

4.2.2.10. External and micro environmental $\mathrm{pH}$ measurement........................... 95

4.2.2.11. Mathematical modeling of dissolution profiles................................... 96

4.2.2.12. Microscopic examination of the structural changes in polymer

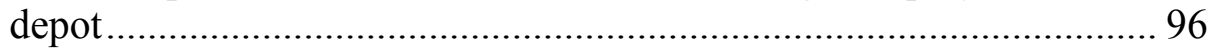

4.2.2.13. Viscosity and injection force measurement ………………................ 96

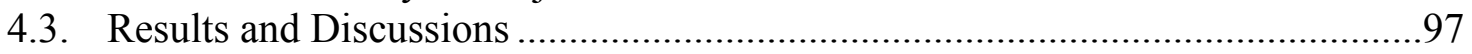

4.3.1. Comparison of micro particle morphology from two different methods......97

4.3.2. Effect of plasticizer and GMO ratio on drug release ……............................ 97

4.3.3. Effect of polymer end group on drug release............................................. 103

4.3.4. Effect of a binary vs. ternary solvent system on drug release .................... 103

4.3.5. Correlation between drug and plasticizer release and lipid structures........ 106

4.3.6. Mathematical modeling of drug release.................................................... 110

4.3.7. Macro and micro environmental $\mathrm{pH}$....................................................... 110

4.3.8. Drug release rate and morphological changes .......................................... 110

4.3.9. Viscosity and injection force................................................................... 116

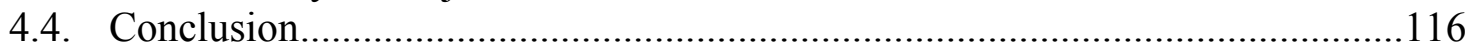

\section{CHAPTER 5. DEVELOPMENT AND CHARACTERIZATION OF PLGA MICRO PARTICLE: POLYCAPROLACTONE CRYSTALLITE BASED

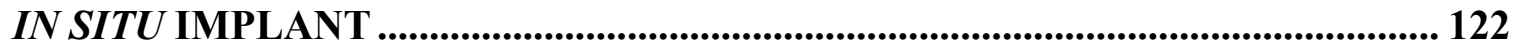

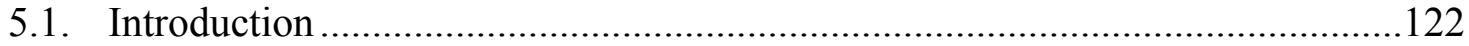

5.1.1. Homogeneous nucleation: birth of primary nuclei .................................... 122

5.1.2. Development of lamellar sheaf: branching of lamellae ............................... 124

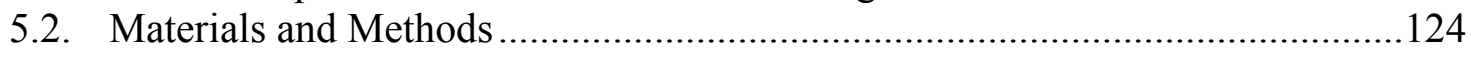

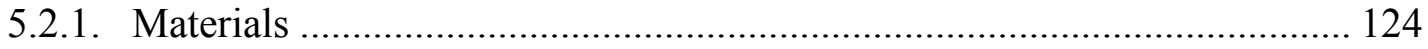

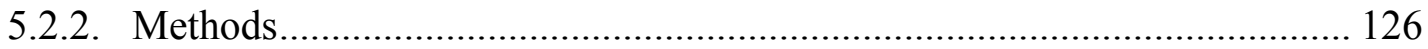

5.2.2.1. PLGA micro particle preparation...................................................... 126

5.2.2.2. PCL crystallites/spherulites preparation ............................................ 126

5.2.2.3. PCL crystallites/spherulites characterization ...................................... 126

5.2.2.3.1. Polarized light microscopy studies ............................................... 126

5.2.2.3.2. Scanning electron microscopy studies ............................................. 126

5.2.2.3.3. Modulated differential scanning calorimetry studies....................... 128

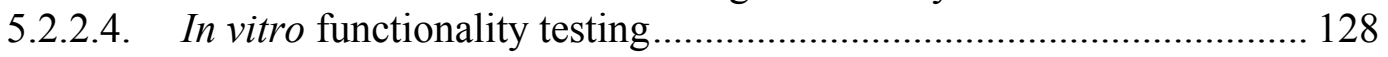

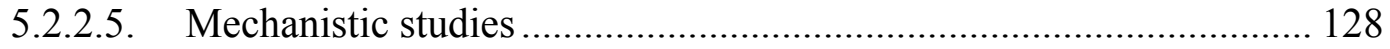

5.2.2.6. Mathematical modeling ................................................................... 128

5.2.2.7. Viscosity and injection force measurement ........................................ 128

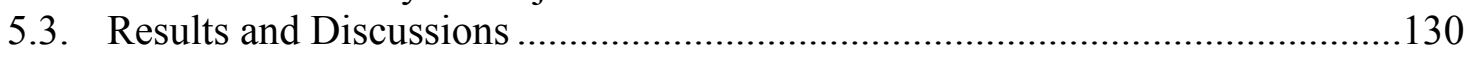

5.3.1. PCL crystallites/spherulites characterization ............................................ 130

5.3.1.1. Polarized light microscopy studies ................................................. 130 
5.3.1.2. Scanning electron microscopy studies .............................................. 130

5.3.1.3. Differential scanning calorimetry studies ......................................... 130

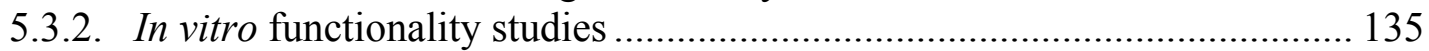

5.3.2.1. Effect of PCL crystallites concentration .......................................... 135

5.3.2.2. Effect of dissolution media volume ................................................... 135

5.3.2.3. Effect of PLGA end group on drug release...................................... 135

5.3.2.4. Effect of micro particle/microsphere morphology on drug release ... 138

5.3.2.5. Effect of buffer concentration of dissolution media ........................... 138

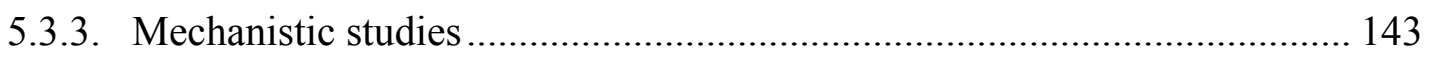

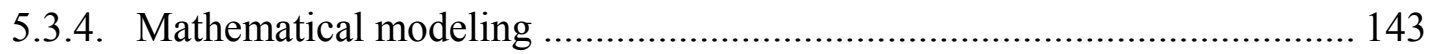

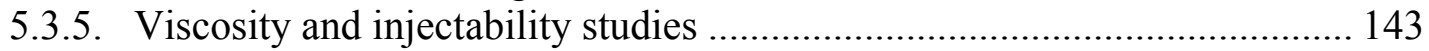

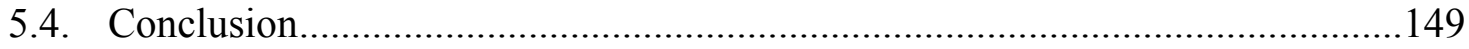

\section{CHAPTER 6. EVALUATION OF BIODEGRADABILITY AND BIOCOMPATIBILITY OF PLGA MICRO PARTICLE: POLYCAPROLACTONE CRYSTALLITE BASED IN SITU IMPLANT ............. 151}

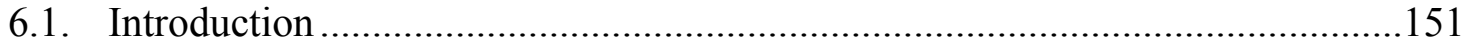

6.1.1. Blood material interactions .................................................................... 151

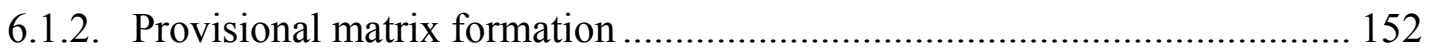

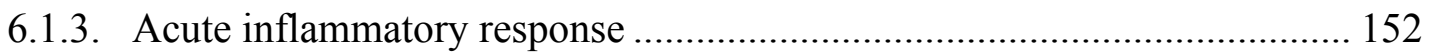

6.1.4. Chronic inflammatory response ............................................................. 154

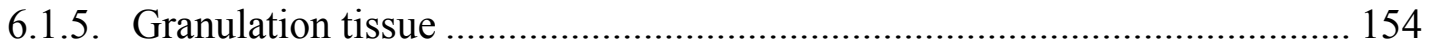

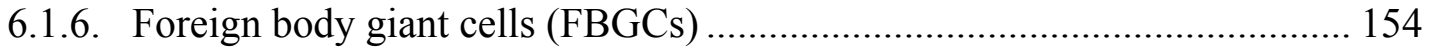

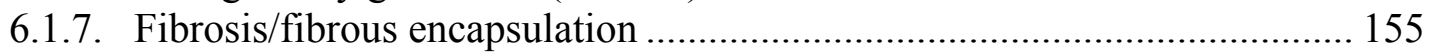

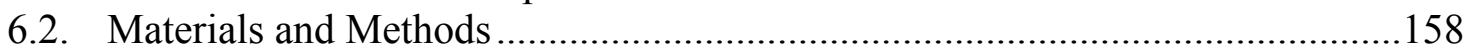

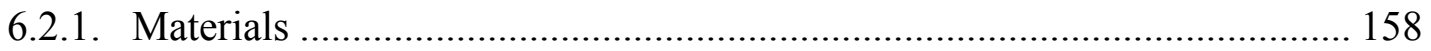

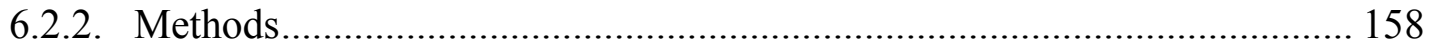

6.2.2.1. Preparation of drug loaded PLGA micro particles............................. 158

6.2.2.2. Determination of drug content in micro particles .............................. 159

6.2.2.3. Preparation of injection vehicle containing PCL crystallites ............. 159

6.2.2.4. Injection of the in situ implant formulation ........................................ 159

6.2.2.5. Preliminary histological preparation ................................................ 159

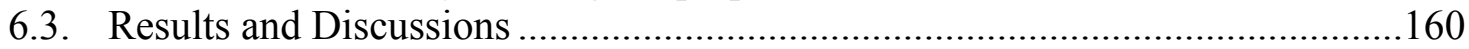

6.3.1. Evaluation of biodegradability of implant .............................................. 160

6.3.2. Biocompatibility of implant .................................................................. 160

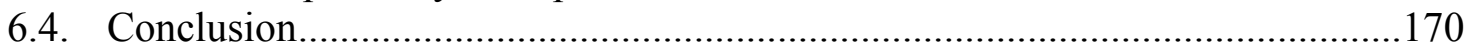

RESEARCH SUMMARY .............................................................................................. 171

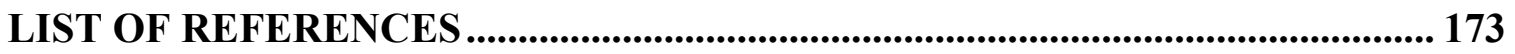

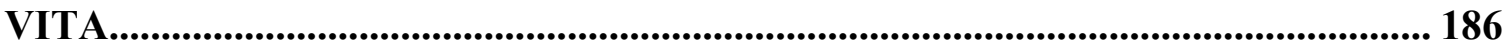




\section{LIST OF TABLES}

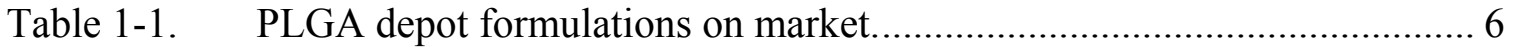

Table 1-2. Limitations of current biodegradable in situ forming drug delivery

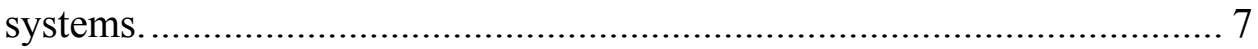

Table 2-1. Formulations used for testing under different dissolution set-ups........... 35

Table 2-2. Buffer composition for dissolution medium......................................... 35

Table 2-3. Intraday and interday variability for leuprolide HPLC assay.................. 38

Table 2-4. $\quad \mathrm{f}_{2}$ values for comparison between various in vitro test methods. ............. 44

Table 2-5. Comparison between 2-piece regression and 3-piece regression methods for correlating in vitro with in vivo drug release.

Table 2-6. Comparison of various in vitro test methods with respect to prediction error by 3 -piece regression model.................................................... 46

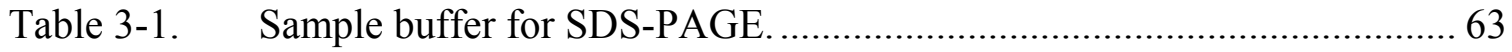

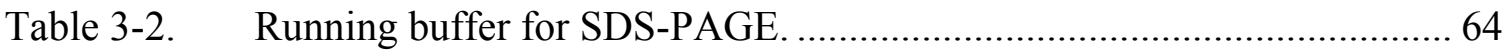

Table 4-1. Drug release rates from PLGA micro particles in presence of different ratio proportions of plasticizer: GMO................................................ 104

Table 4-2. Mathematical equations for modeling drug release............................. 111

Table 4-3. Calculated $\mathrm{R}^{2}$ values from various mathematical models...................... 112

Table 4-4. Comparison of relaxation vs. fickian diffusion for various injection vehicles.

Table 5-1. Calculated $\mathrm{R}^{2}$ values from various mathematical models...................... 146 


\section{LIST OF FIGURES}

Figure 1-1. Burst release mechanism from in situ polymer precipitation systems....... 4

Figure 1-2. Biodegradable injectable polymer classification. ................................. 8

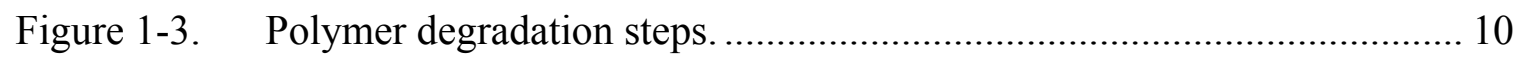

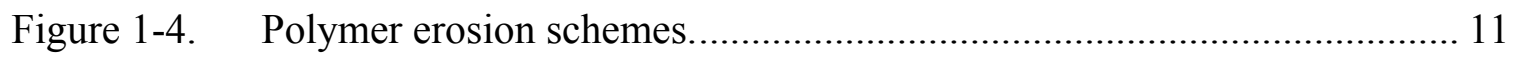

Figure 1-5. PLGA polymer and its metabolic products........................................ 13

Figure 1-6. Variations in micro particle morphology........................................... 17

Figure 1-7. Schematic of micro particle preparation by solvent evaporation

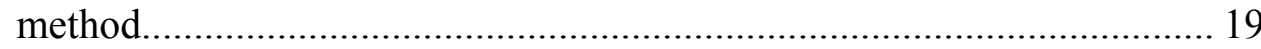

Figure 1-8. Schematic of an $\mathrm{O} / \mathrm{W}$ emulsion solvent evaporation method................... 20

Figure 1-9. Schematic of microsphere preparation by $\mathrm{W} / \mathrm{O} / \mathrm{W}$ multiple emulsion

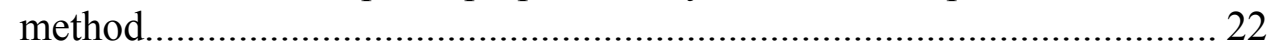

Figure 1-10. Hypothetical drug release profile from PLGA based implants................ 23

Figure 1-11. Components of the proposed injection vehicle....................................25

Figure 1-12. Amino acid sequence of leuprolide acetate. ....................................... 27

Figure 2-1. Visualization of subcutaneous tissue environment around implant.......... 31

Figure 2-2. Schematic representation of PLGA micro particle preparation by film

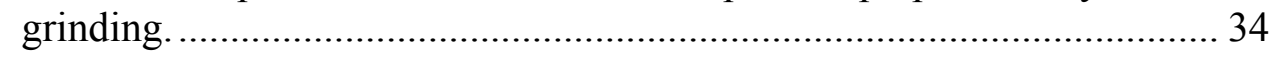

Figure 2-3. Pharmacokinetic model used for fitting of plasma leuprolide concentration data following administration of IV bolus. ..................... 36

Figure 2-4. HPLC standard curve for leuprolide................................................. 38

Figure 2-5. Illustration of set up for in vitro drug release from PLGA formulations using a sample and separate method................................................... 39

Figure 2-6. Illustration of set up for in vitro drug release from PLGA formulations using a Float-A-Lyzer. ............................................................... 40

Figure 2-7. Illustration of set up for in vitro drug release from PLGA formulations using a nylon membrane pouch. 
Figure 2-8. Illustration of set up for in vitro drug release from PLGA formulations using a Teflon cavity with lid. ................................................................. 42

Figure 2-9. In vitro drug release using different experimental set ups and comparison with the deconvoluted plasma profile.

Figure 2-10. Comparison of drug release from PLGA based micro particulate formulation using four different in vitro test methods at the drug loadings of (a) $4 \% \mathrm{w} / \mathrm{w}$ of polymer; (b) $8 \% \mathrm{w} / \mathrm{w}$ of polymer; and (c) $16 \% \mathrm{w} / \mathrm{w}$ of polymer. 50

Figure 2-11. Separation of formulation components in the Float-A-Lyzer................... 51

Figure 2-12. Micro particle aggregates formed at the bottom of the Float-A-Lyzer.... 52

Figure 2-13. Design of the Teflon Cavity used for drug release study (a) side view of the Teflon Cavity; (b) top view of the Teflon cavity; (c) split view of the Teflon cavity; and (d) micro particle aggregates at the bottom of Teflon cavity.

Figure 2-14. Comparison of drug release from PLGA based micro particulate formulation using four different in vitro test methods at PLGA viscosity grades of (a) $0.24 \mathrm{dL} / \mathrm{g}$; and (b) $0.59 \mathrm{dL} / \mathrm{g}$.

Figure 2-15. Comparison of drug release from PLGA based micro particulate formulation containing a single plasticizer (ATEC) using four different in vitro test methods. 55

Figure 3-1. Structures and chemical formula of various vehicle solvents.

Figure 3-2. Liquid-liquid extraction method for determining drug loading in leuprolide containing PLGA micro particles.

Figure 3-3. Two syringe system for in situ PLGA polymeric implant system (IPPIs).

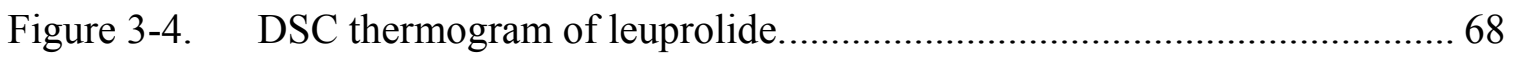

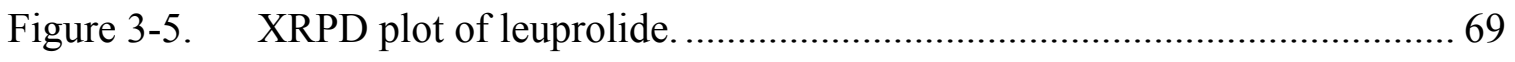

Figure 3-6. XRPD plots of drug-loaded polymer micro particles............................... 70

Figure 3-7. DSC thermograms of (a) PLGA polymer; and (b) drug-loaded polymer micro particles.

Figure 3-8. $\quad$ FT-IR spectra of (a) leuprolide; (b) leuprolide-PLGA-ester physical mixture; (c) drug-loaded PLGA-ester micro particles; and (d) drug-loaded PLGA-acid micro particles. 
Figure 3-9. Visual comparison of drug-loaded polymer mass between (a) polymer with ester end group; and (b) polymer with acid end group...

Figure 3-10. TEAS diagram for leuprolide solubility in various solvents/plasticizers.

Figure 3-11. Leuprolide stability in PBS in absence and presence of $0.2 \%$ sodium azide.

Figure 3-12. Drug stability in PBS in presence of various vehicle solvents/plasticizers.

Figure 3-13. SDS-PAGE result for (a) leuprolide; (b) drug extracted from PLGA-ester micro particles; and (c) drug extracted from PLGA-acid micro particles.

Figure 3-14. LC/MS chromatograms for (a) leuprolide; (b) drug extracted from PLGA-ester micro particles.

Figure 3-15. Representation of polymer $\mathrm{Tg}$ as a function of time during in vitro release study.

Figure 3-16. Comparion between dissolution rate and $\mathrm{Tg}$ as a function of percentage PEG amount in the plasticizer combination with TEC.

Figure 3-17. Comparison between dissolution rate and $\mathrm{Tg}$ as a function of percentage PEG amount in the plasticizer combination with ATEC

Figure 3-18. Comparison between dissolution rate and $\mathrm{Tg}$ as a function of percentage PEG amount in the plasticizer combination with ATBC. ...... 84

Figure 3-19. Response surface plots for (a) Tg; and (b) gel thickness as a function of plasticizer type and percent PEG in the plasticizer composition.

Figure 3-20. Leuprolide release from PLGA micro particles as a function of time in presence of PEG and NMP.

Figure 3-21. Drop in Tg as a function of NMP concentration in the plasticizer composition.

Figure 3-22. Polymer gelling in presence of increasing concentrations of NMP as a function of time.

Figure 4-1. Schematic representation of PLGA microsphere preparation by modified multiple emulsion method. 
Figure 4-2. Microscopic images of PLGA micro particles prepared by (a) film grinding method; (b) and (c) W/O/W modified multiple emulsion method.

Figure 4-3. Surface free drug on PLGA micro particles/microspheres.

Figure 4-4. Ternary phase diagrams for the injection vehicle (a) binary solvent based system; and (b) ternary solvent based system

Figure 4-5. In vitro drug release from PLGA micro particles in presence of various plasticizer: GMO ratios.

Figure 4-6. In vitro drug release from PLGA micro particles prepared by using two different methods.

Figure 4-7. In vitro drug release from PLGA micro particles prepared by using two different end groups.

Figure 4-8. In vitro drug release from PLGA micro particles in presence and absence of the most hydrophobic solvent, EB.

Figure 4-9. Correlation between cumulative drug release and plasticizer release from formulation depot.

Figure 4-10. Various lipid microcrystalline structures formed after equilibration with excess of water of the formulation with following injection vehicle compositions: (a) ATEC:NMP (9:1):GMO (0.2:1); (b) ATEC:NMP (9:1):GMO (0.5:1); and (c) ATEC:NMP (9:1):GMO $(1: 1)$ 108

Figure 4-11. DSC thermograms of various lipidic microcrystalline structures: (a) ATEC:NMP (9:1):GMO (0.2:1); (b) ATEC:NMP (9:1):GMO (0.5:1); and (c) ATEC:NMP (9:1):GMO (1:1).

Figure 4-12. Standard curves of fluorescent dyes for confocal microscopy study...... 113

Figure 4-13. Correlation between micro environmental and external $\mathrm{pH}$ as determined by confocal microscopy.

Figure 4-14. Drug release rate and morphological changes in formulation depot. .... 115

Figure 4-15. Structural changes in formulation depot during drug release.

Figure 4-16. Viscosity studies of the PLGA micro particulate formulation in novel injection vehicle system.

Figure 4-17. Correlation between viscosity and injection force for PLGA solution in the plasticizer. 
Figure 4-18. Comparison of injection force between PLGA solution and PLGA

micro particle suspension in the plasticizer. 120

Figure 4-19. Viscosity of injection vehicle with (a) plasticizer; and (b) plasticizer and glycerol lipid and injection force of injection vehicle with (c) plasticizer; and (d) plasticizer and glycerol lipid.

Figure 5-1. Schematic representation of the hypothetical growth of polymer spherulites.

Figure 5-2. Gibbs free energy of formation of nucleus as a function of its size. ...... 125

Figure 5-3. PCL Crystallite/spherulite preparation process. ....................................... 127

Figure 5-4. Experimental set up for in vitro drug release studies.............................. 129

Figure 5-5. Polarized light microscopic images of PCL crystallites/spherulites (a) before; and (b) after equilibration with excess of water........................ 131

Figure 5-6. Polarized light microscopic images of co-existing PCL crystallites/spherulites and the glycerolipid microcrystalline structures.

Figure 5-7. Scanning electron microscopic images of PCL crystallites at three different magnifications of (a) 50x; (b) 200x; and (c) 3200x.

Figure 5-8. MDSC thermograms of (a) pure PCL polymer; and (b) PCL crystallites/spherulites.

Figure 5-9. Effect of PCL crystallite concentration in injection vehicle on drug release.

Figure 5-10. Effect of volume of dissolution media on drug release from PLGA micro particulate: PCL crystallite formulations at (a) $6 \% \mathrm{w} / \mathrm{w}$ PCL crystallite concentration; (b) $8 \% \mathrm{w} / \mathrm{w}$ PCL crystallite concentration; and (c) $12 \% \mathrm{w} / \mathrm{w}$ PCL crystallite concentration.

Figure 5-11. Effect of PLGA end group on amount of surface adsorbed drug on PLGA micro particles.

Figure 5-12. Effect of PLGA end group on drug release from PLGA micro particulate: PCL crystallite formulation

Figure 5-13. Effect of PLGA micro particle morphology on drug release from PLGA micro particulate: PCL crystallite formulation. 
Figure 5-14. Effect of buffer strength on drug release from PLGA micro particulate: PCL crystallite formulation having PLGA polymer with (a) ester end group; and (b) acid end group.

Figure 5-15. Mechanistic evaluation and correlation between percent drug and percent plasticizer release at two different dissolution media volumes and at PCL crystallite concentrations of (a) $6 \% \mathrm{w} / \mathrm{w}$; (b) $8 \% \mathrm{w} / \mathrm{w}$; and

(c) $12 \% \mathrm{w} / \mathrm{w}$ in injection vehicle.

Figure 5-16. Evaluation of drug release mechanism at various PCL crystallite concentrations and two different dissolution media volumes of (a) 10 $\mathrm{ml}$; and (b) $150 \mathrm{ml}$.

Figure 5-17. Relative relaxation and diffusion coefficients for drug release at various PCL crystallite concentrations.

Figure 5-18. Viscosity and injection forces of injection vehicle in (a) absence; and (b) presence of PCL crystallites. 148

Figure 5-19. Viscosity and injection forces of injection vehicle at various PCL crystallite concentrations of (a) $6 \% \mathrm{w} / \mathrm{w}$; (b) $8 \% \mathrm{w} / \mathrm{w}$; and (c) $12 \%$ $\mathrm{w} / \mathrm{w}$.

Figure 6-1. Permeability of blood capillaries during an inflammatory response...... 153

Figure 6-2. In vivo transition from blood-borne monocyte to biomaterial adherent monocyte/macrophage to foreign body giant cell at the tissue/biomaterial interface.

Figure 6-3. The temporal variation in the acute inflammatory response, chronic inflammatory response, granulation tissue development, and foreign body reaction to implanted biomaterials.

Figure 6-4. Implant remaining at the site of injection at (a) day 7; (b) day 14; (c) day 30; and (d) day 48 post injection.

Figure 6-5. Biodegradation profile for the in situ PLGA micro particles: PCL crystallite implant.

Figure 6-6. Light photomicrograph of subcutaneous tissue 30 days after subcutaneous injection of test formulation consisting of injection vehicle solvent at (a) 10x; (b) and (c) 20x; and (d) 40x magnification. . 163

Figure 6-7. Light photomicrograph of subcutaneous tissue 7 days after subcutaneous injection of test formulation consisting of drug loaded PLGA micro particles dispersed in a PCL crystallite based injection vehicle at (a) and (b) 20x; and (c) and (d) 40x magnification. 
Figure 6-8. Light photomicrograph of subcutaneous tissue 14 days after subcutaneous injection of test formulation consisting of drug loaded PLGA micro particles dispersed in a PCL crystallite based injection vehicle at (a) and (b) 20x; and (c) and (d) 40x magnification

Figure 6-9. Light photomicrograph of subcutaneous tissue 30 days after subcutaneous injection of test formulation consisting of drug loaded PLGA micro particles dispersed in a PCL crystallite based injection vehicle at (a) and (c) 20x; and (b) and (d) 40x magnification.

Figure 6-10. Light photomicrograph of subcutaneous tissue 48 days after subcutaneous injection of test formulation consisting of drug loaded PLGA micro particles dispersed in a PCL crystallite based injection vehicle at $40 \mathrm{x}$ magnification.

Figure 6-11. Average score for the inflammatory tissue response for control and test formulations. 


\section{LIST OF ABBREVIATIONS}

$\alpha$

$\beta$

A

AFM

AIC

ATBC

ATEC

B

DMSO

DSC

DTG

EB

EME

ESEM

$\mathrm{F}$

$\mathrm{f}_{2}$

FDA

FT-IR

GI

GMO

HPLC

IPPI

IV

IVIVC

KD

LC-MS

LCST

LHRH

MDSC

$\mathrm{M}_{\mathrm{i}}$

$\mathrm{M}_{\mathrm{N}}$

MW

MWCO

$\mathrm{N}_{\mathrm{i}}$

NMP

$\mathrm{O} / \mathrm{W}$

PBS

PCL

PD

PEG

PG

PGA

PHA
Macro constant for drug distribution phase

Macro constants for drug elimination phase

Macro constants for drug distribution phase

Atomic force microscopy

Akaike information criteria

Acetyl tributyl citrate

Acetyl triethyl citrate

Macro constants for drug elimination phase

Dimethyl sulfoxide

Differential scanning calorimetry

Derivative thermo gravimetry

Ethyl benzoate

Emulsified micro emulsion

Environmental scanning electron microscope

Fickian diffusion coefficient

Similarity factor

Food and drug administration

Fourier-transform infrared spectroscopy

Gastrointestinal

Glyceryl monooleate

High pressure liquid chromatography

In situ PLGA polymeric implant

Intrinsic viscosity

In vitro-in vivo correlation

Kilo Daltons

Liquid chromatography mass spectrometry

Lower critical solution temperature

Leuprorelin hormone releasing hormone

Modulated differential scanning calorimetry

Molecular weight of the ith component

Number average molecular weight

Molecular weight

Molecular weight cut off

Number of moles of the ith component

$\mathrm{N}$-methyl pyrrolidone

Oil in water

Phosphate buffer saline

Polycaprolactone

Polydispersity

Polyethylene glycol

Propylene glycol

Poly glycolic acid

Poly hydroxyalkanoate 


$\begin{array}{ll}\text { PLA } & \text { Poly lactic acid } \\ \text { PLGA } & \text { Poly lactide-co-glycolide } \\ \text { Poly (NIPAAM) } & \text { Poly (N-isopropyl acrylamide) } \\ \text { PVP } & \text { Polyvinyl pyrrolidone } \\ \text { R } & \text { Polymer chain relaxation coefficient } \\ \text { R }^{2} & \text { Regression coefficient } \\ \text { RO } & \text { Reverse osmosis } \\ \text { RP } & \text { Reverse phase } \\ \text { RSD } & \text { Relative standard deviation } \\ \text { SDS-PAGE } & \text { Sodium dodecyl sulphate polyacrylamide gel electrophoresis } \\ \text { SEM } & \text { Scanning electron microscopy } \\ \text { TA } & \text { Thermal analysis } \\ \text { TEC } & \text { Triethyl citrate } \\ \text { Tg } & \text { Glass transition temperature } \\ \text { TG } & \text { Thermo gravimetry } \\ \text { THF } & \text { Tetrahydrofuran } \\ \text { W/O } & \text { Water in oil } \\ \text { W/O/W } & \text { Water in oil in water } \\ \text { W } & \text { Weight of the ith component } \\ \text { XRPD } & \text { X-ray powder diffraction }\end{array}$




\section{CHAPTER 1. INTRODUCTION}

The method by which a drug is delivered can have a significant effect on its efficacy. Some drugs have an optimum concentration range within which maximum benefit is derived and concentrations above or below this range can be toxic or produce no therapeutic benefit at all. To achieve this optimum concentration of drug in the body, the drug needs to reach the blood stream and further needs to arrive at the site of action in order to show any efficacy. Oral route of drug administration, although considered to be the most preferred route due to patient compliance, it is not always possible. Some difficult and more complex drug molecules require direct administration into the blood stream thereby bypassing the oral route of absorption. The scientific advances in disease treatment have resulted in a greater focus on the development and production of biological molecules such as peptides, proteins and antibodies etc. Such complex drug molecules face an immense challenge in terms of chemical stability as well as absorption if administered orally. The clusters of enzymes present in the gastrointestinal (GI) tract rip the drug molecule apart much before the actual absorption process resulting in inefficacious blood concentrations of the drug. This calls for an alternate route of drug delivery that can be relied upon for delivery of such complex drug molecules.

Further, certain chronic disease conditions demand a continuous drug delivery to the patient along with improved patient compliance. Injectable controlled drug delivery systems can play a crucial role in treatment/prophylaxis of chronic disease states as they can be designated for a significantly longer duration than the oral administration. Parenteral drug delivery systems were first reported in the mid- $19^{\text {th }}$ century by Alexander Wood. Since then a number of technological advances have been made in the area of parenteral drug delivery leading to the development of sophisticated systems that allow drug targeting and the sustained or controlled release of parenteral medicines. One of the recent areas of controlled drug delivery through parenteral route includes injectable in situ forming drug delivery systems.

\subsection{Biodegradable Injectable In Situ Forming Drug Delivery Systems}

Development of injectable drug delivery systems has received noticeable attention in past few years. Past advancements include delivery systems such as emulsions, ${ }^{1-3}$ liposomes, ${ }^{4-7}$ biodegradable micro polymeric systems, due in part to rapid clearance by spheres $^{8-10}$ and micelles. ${ }^{11,12}$ Although such formulations have provided considerable advantages towards efficient drug delivery, they are associated with certain limitations. Emulsions have limitations as long acting formulations due to the associated stability issues of loss of structure by dilution with the surrounding body fluid. ${ }^{13}$ Liposomes face a challenge for long term drug delivery due to their rapid clearance by macrophages and other cells. ${ }^{14,15}$ Microspheres have inherent disadvantages such as complex manufacturing processes and a high percentage of surface adsorbed drug that results in a burst release of drug and toxicity. Stability of the micelles is essentially determined by the critical micelle concentration (CMC) of the surfactant molecules resulting in dilution 
of the formulation and breaking apart of micelles once the surfactant concentration falls below the CMC. ${ }^{16,17}$

The injectable in situ forming drug delivery systems are therefore being developed with the idea of overcoming the shortcomings in currently known injectable formulations. $^{18-20}$ The characteristics of some of the most common in situ forming drug delivery systems are as follows:

\subsubsection{Thermoplastic pastes}

Thermoplastic polymers form a semi-solid mass upon cooling to the body temperature after injecting in the molten form. They are characterized as having a low melting point or glass transition temperature $(\mathrm{Tg})$ in the range of $25-65^{\circ} \mathrm{C}$ and an intrinsic viscosity (IV) in the range of $0.05-0.8 \mathrm{dl} / \mathrm{g} .{ }^{21}$ Viscosity plays a critical role as thermoplastic pastes with viscosities lower than $0.05 \mathrm{dL} / \mathrm{g}$ do not show any delayed release effect whereas those with viscosities above $0.8 \mathrm{dL} / \mathrm{g}$ are not injectable using a needle. Monomers such as D,L-lactide, glycolide, E-caprolactone, and orthoesters can be used for preparation of thermoplastic pastes. ${ }^{22,23}$ Thermoplastic pastes are biocompatible and biodegradable and have applications in carriers of pharmaceutical compounds, ${ }^{24}$ surgical sutures, ${ }^{25}$ ocular implants ${ }^{26}$ and soft tissue repair. ${ }^{27,28}$

\subsubsection{In situ cross-linked polymer systems}

In situ cross-linked polymeric systems have applications in sustained drug release due to formation of a cross linked network of polymer chains at the site of injection. The cross-linking is essentially accomplished by free radical reactions initiated by heat or absorption of photons, or ionic interactions between small cations and polymer anions.

\subsubsection{Thermosets}

Thermosets are the polymers that have low viscosity and are free flowing during the formation process but set into a permanent shape upon heating. The process of heating is known as "curing" which essentially supplies the energy required for crosslinking. The controlled release properties of such polymeric systems are therefore due to the rigid structure formed upon curing. However, certain inherent limitations of these polymeric systems such as stringent reaction conditions for in vivo applications and requirements for non-toxic monomers and solvents do not allow them to be used extensively for pharmaceutical drug delivery. ${ }^{29,30}$ 


\subsubsection{Photo cross-linked gels}

These polymers that form photo cross-linked gels are introduced at the site of injection and then photo cured in situ with fiber optic cables. ${ }^{30}$ The polymers have a liquid or moldable nature and are rapidly cross linked compared to the thermosets. Delivery of various proteins from a polyethylene glycol-poly lactic acid (PEG-PLA) hydrogel illustrates the drug delivery capabilities of this approach. ${ }^{31}$

\subsubsection{Ion mediated gelation}

Polymers such as alginates have the capability to form a gel upon contact with divalent cations such as calcium ions. These polymers can therefore be directly used as drug carriers or carriers for other delivery systems e.g. liposomes. ${ }^{32}$ One of the most common examples is the calcium loaded liposomes administered along with sodium alginate in the form of a fluid suspension. The calcium ions are released when the system gets heated to body temperature and interact with the alginate polymer resulting in the formation of a gel that controls the drug release.

\subsubsection{In situ polymer precipitation}

\subsubsection{Solvent-removal precipitation}

Such a system involves dissolving a biodegradable polymer such as polylactide-co-glycolide (PLGA) in a solvent that also acts as a polymer plasticizer. The polymeric solutions contain either dissolved or dispersed drug, which upon injection into the subcutaneous or intramuscular tissue results in the leaching of the solvent into surrounding tissue and precipitation of the polymer. Such a system has been developed for various peptide drugs e.g. Eligard ${ }^{\circledR}$ based on Atrix technology of in situ solvent removal and polymer precipitation containing leuprolide acetate as the active. Delivery systems based on solvent removal and polymer precipitation, however, presents three basic limitations:

- A huge burst release of drug.

- A tri-phasic drug release pattern.

- High viscosity of the polymer solution resulting in painful injections.

The mechanism for huge burst and tri-phasic release of drug is depicted in Figure 1-1. Essentially, the solvent, while diffusion out into the surrounding tissue carries a major percentage of the drug with it resulting in a high initial drug release. Further since the formulation when administered is a liquid and it takes some time for it to turn into a gel. This lag period provides enough time for the drug to leave out resulting in huge initial plasma drug concentrations. Most commonly used solvents in this approach 


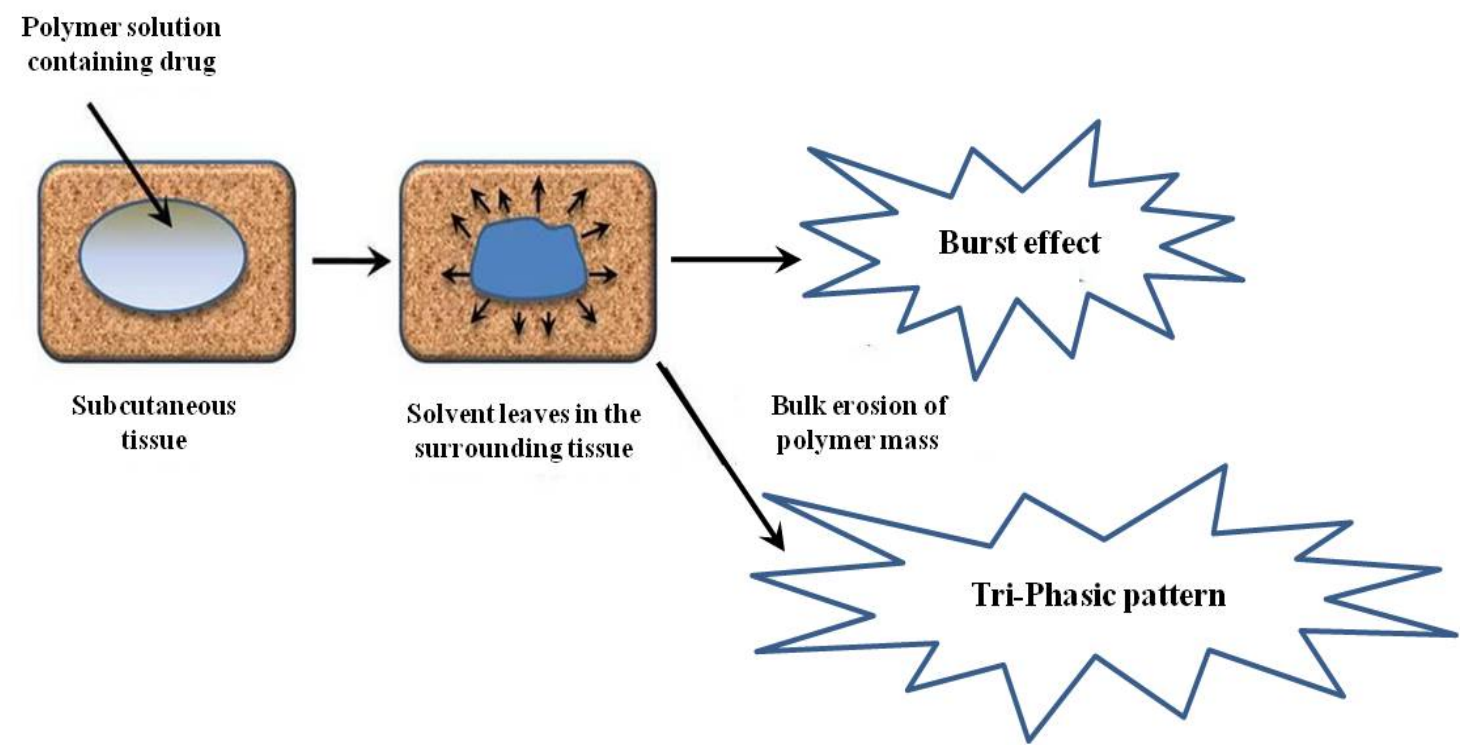

Figure 1-1. Burst release mechanism from in situ polymer precipitation systems. 
include N-methyl pyrrolidone (NMP), propylene glycol (PG), acetone, dimethyl sulfoxide (DMSO), tetrahydrofuran (THF) and triacetin. The most preferred ones are, however, NMP and DMSO due to their pharmaceutical precedence. ${ }^{33}$ Table 1-1 shows the in situ forming depot systems formulated with PLGA polymer currently available on market. $^{34}$

\subsubsection{Thermally induced sol-gel transitions}

Certain polymers exhibit the capacity to undergo a solubility change with temperature thereby transforming from a sol to a gel state that is responsible for controlled drug release. ${ }^{35-37}$ The transformation from a sol to a gel like structure takes place upon injection of the polymeric system into the body. Poly (N-isopropyl acrylamide) [poly (NIPAAM)] and pluronics tri-block copolymers are the examples of thermo sensitive polymers and they exhibit the unique phenomenon of a lower critical solution temperature (LCST).

\subsubsection{In situ solidifying organogels}

The organogels are essentially water insoluble amphiphilic lipids that form various types of lyotropic liquid crystals upon equilibration with excess of water. The commonly used amhiphilic lipids include glyceryl esters of fatty acids such as glyceryl monoleate, glyceryl monopalmitostearate, glyceryl monolinoleate etc. Such lipids form cubic liquid crystalline structures that are used for sustained drug delivery due to their high viscosities. ${ }^{38-40}$ Such systems are completely biodegradable and the biodegradation occurs through the action of lipases. ${ }^{41}$ A significant amount of work has been done to date in the area of in situ forming drug delivery systems with numerous drug delivery applications. However, there are certain limitations associated with the aforementioned systems and are highlighted in Table 1-2. ${ }^{42}$

\subsection{Biodegradable Polymers}

Talking of the parenteral sustained or controlled drug delivery systems, one of the utmost desirable features is biodegradability of the polymer used for drug delivery. Back in 1920 s, when polymers were synthesized from glycolic acid, polymer degradation was not one of the desired features where properties and performance deteriorated with time. The concept of polymer degradation, however, has changed completely over a period of time with polymer degradation being one of the most desired characteristics of the polymers used for injectable drug delivery. Polymer degradability apart from offering tremendous potential as the basis for controlled drug delivery also helps avoid a surgical procedure to remove the remaining polymer from the body after the course of treatment.

Based on the source of origin, biodegradable polymers are categorized as natural and synthetic polymers. Figure 1-2 shows the two polymer classes with some common 
Table 1-1. PLGA depot formulations on market.

\begin{tabular}{|c|c|c|c|}
\hline Product & Active ingredient & Manufacturer & Formulation \\
\hline Lupron Depot ${ }^{\circledR}$ & Leuprolide acetate & TAP Inc. & Micro particles \\
\hline Nutropin Depot $\AA$ & Growth hormone & Genetech & Micro particles \\
\hline Suprecur ${ }^{\circledR}$ MP & Buserelin acetate & Aventis & Micro particles \\
\hline Decapeptyl $\AA$ & Triptorelin pamoate & Ferring & Micro particles \\
\hline Sandostatin LAR $₫$ Depot & Octreotide acetate & Novartis & Micro particles \\
\hline Somatuline $\AA$ LA & Lanreotide & Ipsen & Micro particles \\
\hline Trelstar $^{\mathrm{TM}}$ Depot & Triptorelin pamoate & Pfizer & Micro particles \\
\hline Vivitrol ${ }^{\circledR}$ & Naltrexone & Alkermes & Micro particles \\
\hline Profact ${ }^{\circledR}$ Depot & Buserelin acetate & Aventis & Implant \\
\hline Zoladex ${ }^{\circledR}$ & Goserelin acetate & Astrazeneca & Implant \\
\hline Eligard $\AA$ & Leuprolide acetate & $\begin{array}{c}\text { Sanofi } \\
\text { Synthelabo }\end{array}$ & $\begin{array}{l}\text { In situ forming } \\
\text { Implant }\end{array}$ \\
\hline
\end{tabular}


Table 1-2. Limitations of current biodegradable in situ forming drug delivery systems.

\section{Delivery system}

Thermoplastic pastes

In situ cross-linked systems

Thermosets

Photocross-linked gels

Ion mediated gelation

\section{In situ polymer precipitation}

Solvent induced precipitation

Thermally induced sol-gel transition

\section{Organogels}

High temperature at the time of injection

Unacceptable level of heat released during reaction

Burst in drug release

Toxicity of unreacted monomers

Shrinkage and brittleness of the polymer due to high degree of cross linking

Low shelf life

Burst in drug release

Long degradation time

Burst in drug release

Utilization of organic solvents

Burst in drug release

Stability of oil and purity of waxes

Lack of toxicity data

Phase separation 


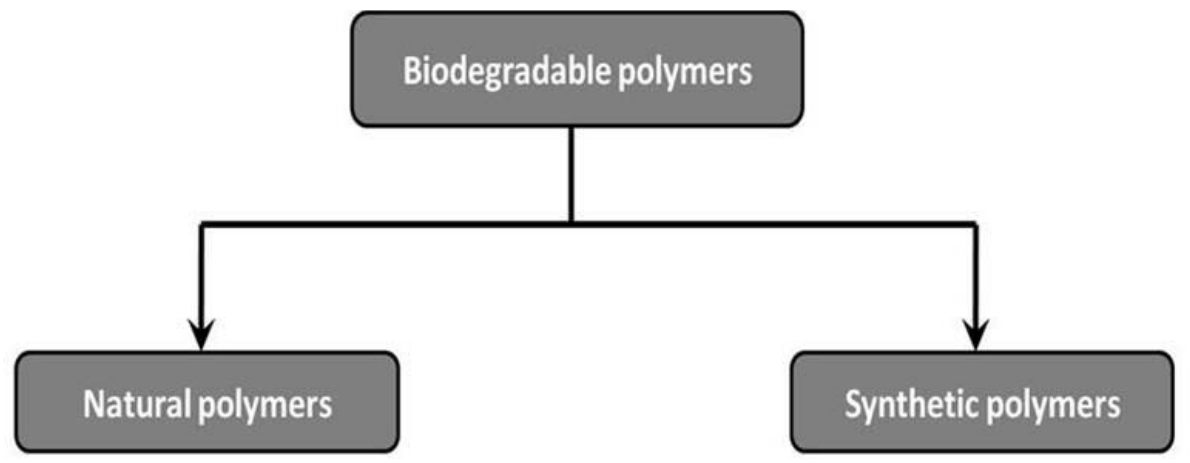

-Fibrin

- Collagen

-Chitosan

- Gelatin

-Hyaluronan ...
-PLA, PGA, PLGA, PCL, Polyorthoesters...

-Poly(dioxanone)

-Poly(anhydrides)

- Poly(trimethylene carbonate)

-Polyphosphazenes...

Figure 1-2. Biodegradable injectable polymer classification. 
examples. Despite of the availability of many naturally existing biodegradable polymers, major emphasis has been on the synthetic polymers. This is due to several different reasons such as the tailor able properties, predictable lot to lot uniformity, less concerns of immunogenicity and reliability of the source of raw material for the synthetic polymers. There are two major degradation mechanisms that have been characterized for the currently known synthetic biodegradable polymers. These include enzymatic degradation and hydrolysis. The degree and rate of hydrolysis depends on the main polymer chain structure and acids show a faster rate of degradation than esters. Such variation in the rate of polymer degradation allows the selection of the most appropriate polymer that would control the drug release for a certain period of time. Polymer degradation can be divided into four steps as depicted in Figure 1-3. ${ }^{43}$

Furthermore, certain biodegradable polymers undergo a surface erosion process, whereby, the polymer sample is eroded from the surface and the overall mass loss is much faster than the entry of water into the bulk. Examples of synthetic polymers that exhibit surface erosion include poly (ortho) esters and polyanhydrides. The second scheme of degradation is the bulk erosion where degradation takes place throughout the whole body of the polymer sample and entry of water is faster than the rate of polymer degradation, ${ }^{44}$ The diagrammatic representation of surface and bulk erosion processes is shown in Figure 1-4. ${ }^{4}$

Factors that influence polymer degradation behavior include:

- Chemical structure and chemical composition

- Distribution of repeat units in multimers

- Molecular weight (MW)

- Polydispersity (PD)

- Presence of low molecular weight (MW) Compounds (monomer, oligomers, solvents, plasticizers, etc.)

- Presence of ionic groups

- Presence of chain defects

- Presence of unexpected units

- Configurational structure

- Morphology (crystallinity, presence of microstructure, orientation and residue stress)

- Processing methods \& conditions

- Method of sterilization

- Annealing 


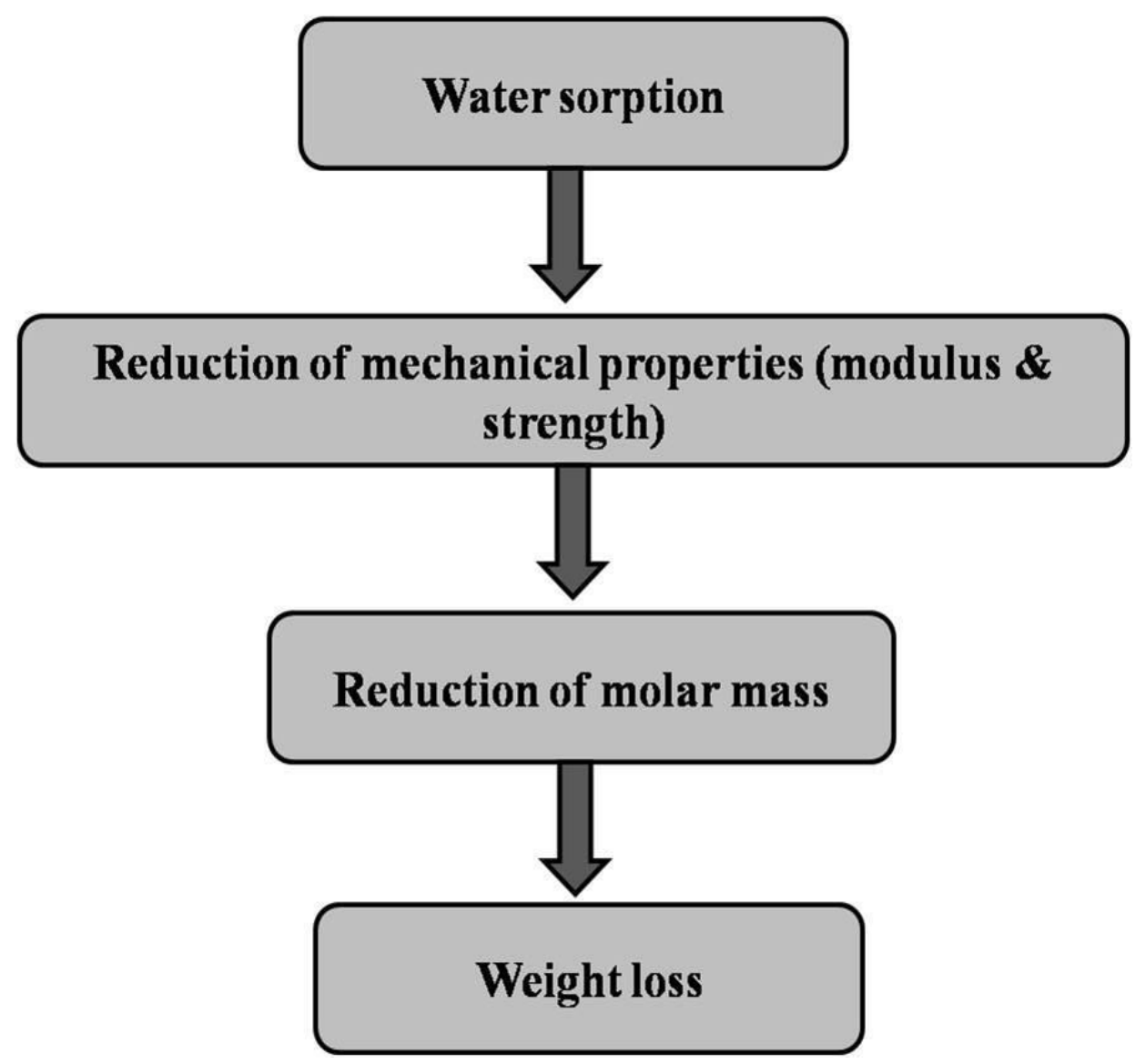

Figure 1-3. Polymer degradation steps. 


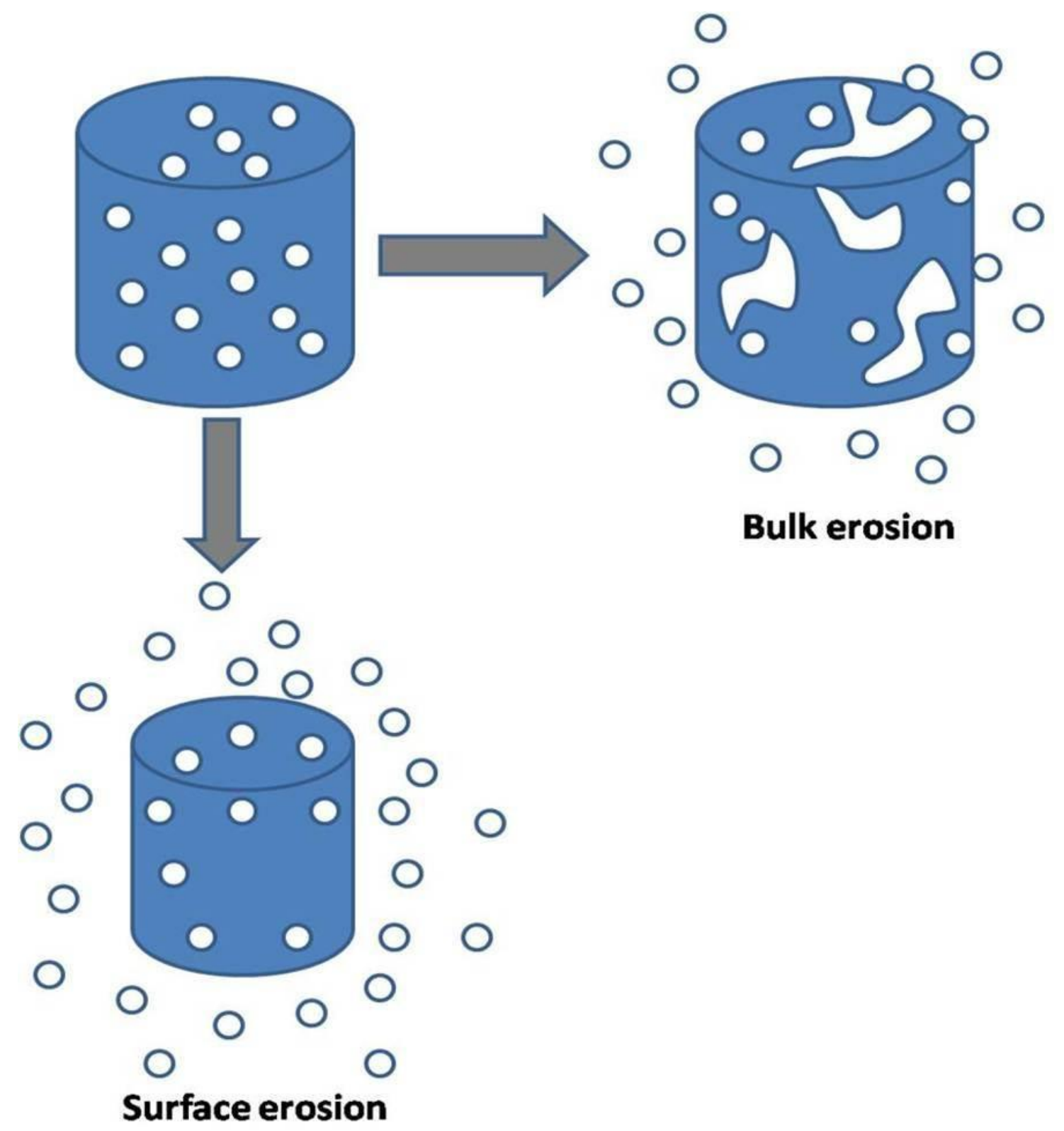

Figure 1-4. Polymer erosion schemes. 
- Storage history

- Site of implantation

- Absorbed compounds

- Physiochemical factors (shape, size)

- Mechanism of hydrolysis (enzymes vs. water)

\subsubsection{Polyesters PLA and PLGA}

Aliphatic polyester polymers such as poly lactic acid (PLA) and poly lactide-co-glycolide (PLGA), which is the copolymer of poly lactic acid with poly glycolic acid (PGA) polymer, are the most commonly known and well used polymers among various biodegradable polymers known for parenteral drug delivery. This is due to their inherent characteristics such as excellent biodegradability and biocompatibility. ${ }^{45-47}$ These polymers degrade by non-enzymatic hydrolysis of ester bond in the body resulting in formation of metabolic products such as lactic acid and glycolic acid (Figure 1-5). ${ }^{48} \mathrm{~A}$ number of groups have publishes excellent work on PLGA polymeric based drug delivery systems. Various polymeric devices such as implants, microspheres, microcapsules, nanoparticles have been designed using these polymers.

\subsubsection{Biodegradation of PLGA polymer}

PLGA polymers undergo chain scission at the ester linkage in an aqueous environment both in vitro as well as in vivo. The polymer undergoes a bulk degradation process with the degradation usually occurring as a faster rate in the core of polymer matrix due to accumulation of acidic degradation by-products. More carboxylic acid groups appear on the polymer chains as they undergo random chain scission. These carboxylic acid groups create an acidic micro-environment around the individual PLGA polymer chains thereby catalyzing the degradation reaction. The amorphous regions in the polymer chains degrade faster than the crystalline regions. The degradation rate of PLGA polymers is determined by various factors such as molar ratio of lactic acid and glycolic acid in the polymer chains, molecular weight (MW) of the polymer, degree of crystallinity and glass transition temperature $(\mathrm{Tg})$ of the polymer. ${ }^{49-57}$

A three phase degradation mechanism has been proposed for PLGA:

(1). Random chain scission process: The MW of the polymer decreases significantly without appreciable weight loss and no soluble monomer products are formed.

(2). Middle phase: A decrease in MW is accompanied by a rapid loss of mass along with the formation of soluble oligomeric and monomer products.

(3). Complete polymer solubilization: Monomer products are formed from the 


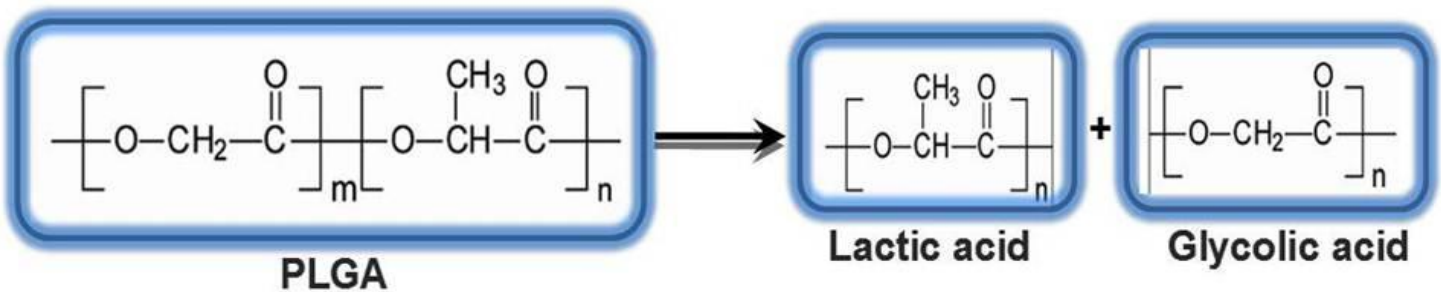

Figure 1-5. PLGA polymer and its metabolic products. 
larger oligomeric fragments. ${ }^{58}$ These monomeric products are more soluble.

\subsubsection{Biocompatibility of PLGA polymer}

The PLGA polymer has low immunogenicity, minimal toxicity, and excellent biocompatibility and biodegradation characteristics. These characteristics made these polymers excellent candidates for biomedical applications such as ligament reconstruction, tracheal replacement, surgical dressings, vascular grafts and fracture repairs. ${ }^{59,60}$ PLGA microspheres have known to induce mild immune response such as infiltration of macrophages, neutrophils, fibroblasts and some lymphocytes; however, the response subsides gradually over time. ${ }^{61,62}$

\subsubsection{Physicochemical properties of PLGA}

The overall biodegradation behavior of PLGA polymers depends on their physicochemical properties such as molecular weight, glass transition temperature and copolymer ratios. Numerous techniques are available to understand and characterize the aforementioned properties of PLGA polymer and this information helps in designing the delivery systems based on PLGA polymers with desired drug release characteristics.

\subsection{Molecular weight and polydispersity}

The average molecular weight can be calculated by different ways. The type of molecular weight estimation on any given polymer sample depends on the property of the polymer to be studied. For example, high molecular weight molecules may contribute more towards the strength of polymer than low molecular weight molecules and thus the average molecular weight for strength properties should be weighted to highlight the presence of high molecular weight polymer. The number average molecular weight $\left(\mathrm{M}_{\mathrm{N}}\right)$ of a polymer is represented by Equation 1-1.

$$
\overline{\mathrm{M}_{\mathrm{N}}}=\frac{\sum_{\mathrm{i}=1}^{\infty} \mathrm{N}_{\mathrm{i}} \mathrm{M}_{\mathrm{i}}}{\sum_{\mathrm{i}=1}^{\infty} \mathrm{N}_{\mathrm{i}}}=\frac{\text { Total weight }}{\text { Number of polymers }}=\frac{\text { Weight }}{\text { Polymer }}
$$

The weight average molecular weight $\left(\mathrm{M}_{\mathrm{W}}\right)$ on the other hand, is represented by the Equation 1-2 as follows:

$$
\overline{\mathrm{M}_{\mathrm{N}}}=\frac{\sum_{\mathrm{i}=1}^{\infty} \mathrm{N}_{\mathrm{i}} \mathrm{M}_{\mathrm{i}}^{2}}{\sum_{\mathrm{i}=1}^{\infty} \mathrm{N}_{\mathrm{i}} \mathrm{M}_{\mathrm{i}}}
$$

Polydispersity (PD), is therefore represented as the ratio between the weight average and number average molecular weights (Equation 1-3). ${ }^{63}$ 


$$
\mathrm{PD}=\frac{\mathrm{M}_{\mathrm{W}}}{\mathrm{M}_{\mathrm{N}}}
$$

Where $\mathrm{N}_{i}=$ number of moles of the $i^{\text {th }}$ component, $\mathrm{M}_{i}=$ the molecular weight of the $i^{\text {th }}$ component, $\mathrm{W}_{i}=$ weight of the $i^{\text {th }}$ component.

\subsection{Optical activity and crystallinity}

PLA can exist in two stereo forms due to an asymmetric carbon atom in lactic acid molecule, known as an optically active form L-poly lactic acid (L-PLA) and optically inactive racemic form (DL-PLA). PLGA prepared from L-PLA and poly glycolic acid (PGA) are crystalline co-polymers while those from DL-PLA and PGA are amorphous in nature. ${ }^{58}$ It has been reported that PLGAs containing $>70 \%$ glycolide are amorphous in nature and the degree of crystallinity and melting point of the polymers are directly related to the MW of these polymers. ${ }^{64}$ The properties affected by the crystallinity of PLGA polymers include mechanical strength, swelling behavior, capacity to undergo hydrolysis and the rate of biodegradation. The crystallinity of PLGA polymer in turn is further dependent on the type and molar ratio of the two individual monomer components (lactide and glycolide) in the polymer chain.

\subsection{Glass transition temperature $(\mathrm{Tg})$}

$\mathrm{Tg}$ is the temperature at which the polymer changes from a glassy state to a more rubbery state. At this temperature, the polymer changes from a brittle structure to a more plastic structure. The Tg of PLGA polymers is above the normal body temperature of $37^{\circ} \mathrm{C}$ and therefore, it is easy to fabricate these polymers into appropriate delivery devices. The most common technique to determine the $\mathrm{Tg}$ of a polymer is differential scanning calorimetry (DSC) or modulated differential scanning calorimetry (MDSC). The Tg of PLGA polymer decreases with a decrease in percent lactide content and a decrease in $\mathrm{MW}$ of the polymer. ${ }^{65}$

\subsection{Biodegradable Polymer Devices}

\subsubsection{Implants}

The implants are polymer sticks about 1 centimeter long with a diameter of about 1 millimeter. Physicians apply them with a commercially available standard syringe under the skin. The implants are placed under the skin into the subcutaneous tissue with the help of a large bore needle (trocar). ${ }^{66}$ The biggest advantage of polymeric implants is the long term drug delivery with patient compliance. However, the most common limitation of such implants is painful injection ${ }^{48}$ and also the degradation of drug within the polymer matrix during implant fabrication procedure. The common techniques for 
implant preparation are melt extrusion, ${ }^{67}$ compression $^{68}$ and injection molding. ${ }^{69}$ Most of the polymer implants are manufactured by dispersing the drug into the polymer melt and therefore, the high temperatures required to melt certain polymers could be detrimental to drug stability. 69

Despite of the aforementioned limitations, the implants are currently being prepared for various drugs e.g. insulin, leuprolide, somatostatin analogue ${ }^{69}$ etc. The double-layered implant consisting of a polymer matrix containing insulin and a poly lactic acid layer which was coated partially on one of the surfaces of the insulin-polymer matrix was prepared by Yamakawa and co-workers. ${ }^{70}$ The octreotide implant is available in the market as a soft, flexible 6-monthhydrogel implant based on a patented HYDRON Polymer Technology. PLGA based implant systems for controlled delivery of leuprorelin hormone releasing hormone (LHRH) agonists are available on the market under the brand names Zoladex ${ }^{\circledR}$ and Profact ${ }^{\circledR}$ for treatment of prostate cancer.

\subsubsection{Micro particles}

Micro particles have been studied extensively by various researchers as a means of controlled injectable drug delivery. Micro particles can be of any morphology depending on the method of preparation i.e. microcapsules, microspheres or micro particles (Figure 1-6). ${ }^{71}$ Microcapsules and microspheres are more uniform and spherical in geometry than micro particles. Micro particles can be fabricated with size ranging from 1 to 1000 microns. Particles $<125$ microns in diameter are, however, acceptable for injection purposes. ${ }^{46}$ The most crucial factor in the design of injectable microspheres is the choice of an appropriate biodegradable polymer. The release of the drug molecule from biodegradable microspheres is controlled by diffusion through the polymer matrix and polymer degradation. Other polymer characteristics such as composition of copolymer ratios, polymer crystallinities, glass-transition temperature, and hydrophilicities also play a critical role in the drug release process. Apart from the aforementioned microsphere and polymer characteristics, the possible mechanisms of drug release from microspheres are as follows: initial release from the surface, release through the pores, and diffusion through the intact polymer barrier, diffusion through a water-swollen barrier, polymer erosion, and bulk degradation. All these mechanisms together play a part in the release process. ${ }^{72}$

Microspheres are prepared by numerous techniques such as spray drying, solvent evaporation, phase separation (co-acervation), spray congealing etc. The microsphere preparation should satisfy certain criteria:

- The ability to incorporate reasonably high concentrations of the drug.

- Stability of the preparation after synthesis with a clinically acceptable shelf life.

- Controlled particle size and dispersability in the vehicles for injection.

- Release of active reagent with a good control over a wide time scale. 


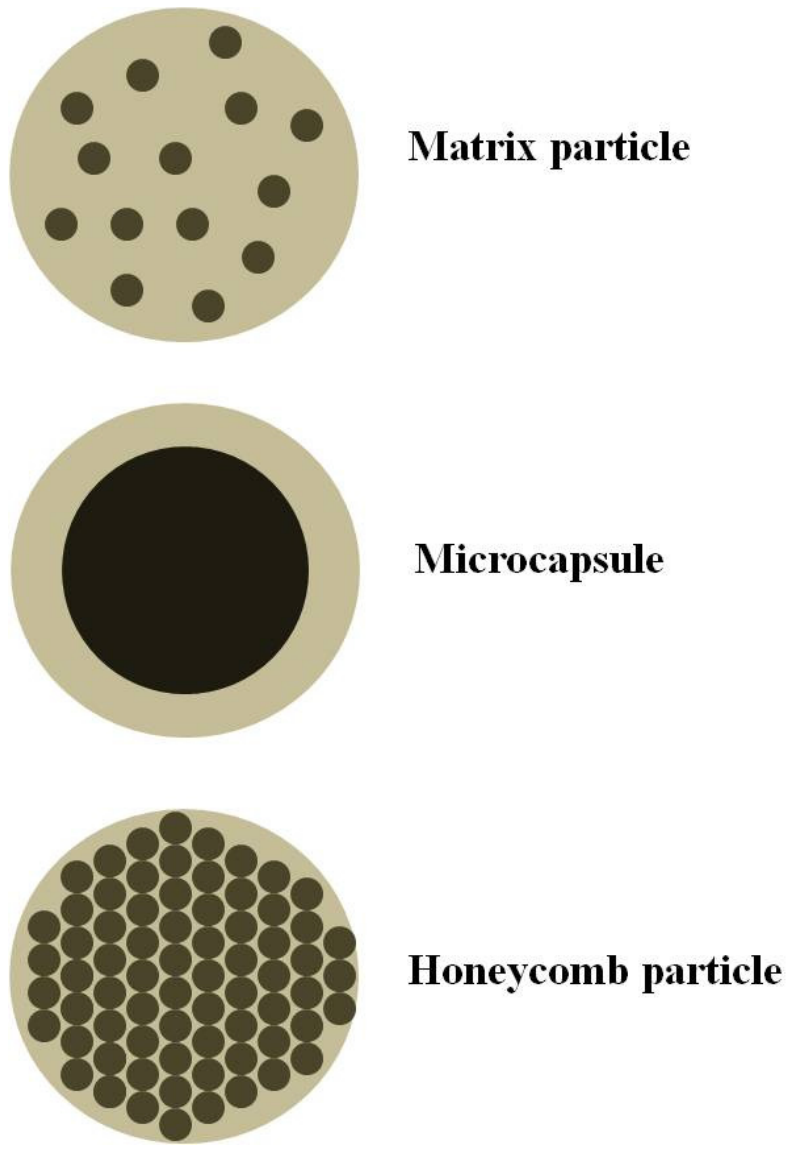

Figure 1-6. Variations in micro particle morphology. 
- Biocompatibility with a controllable biodegradability and susceptibility to chemical modification.

- Produce micro particles with good flowability and syringeability.

\subsubsection{Solvent evaporation}

The solvent evaporation method to produce microspheres of poly lactic acid (PLA), and its co-polymer poly lactide-co-glycolide (PLGA) has been studied extensively due to the biocompatibility of these polymer. ${ }^{73-77}$ The polymer is dissolved in a suitable water immiscible solvent, and the medicament is dispersed or dissolved in this polymeric solution.

The resultant solution or dispersion is then emulsified in an aqueous continuous phase to form discrete droplets. ${ }^{78}$ The solvent evaporation method can be further classified into water in oil $(\mathrm{W} / \mathrm{O})$, oil in water $(\mathrm{O} / \mathrm{W})$ or water in oil in water $(\mathrm{W} / \mathrm{O} / \mathrm{W})$ multiple emulsion method depending on the state of drug in polymer solution and the type of dispersion medium (Figure 1-7).

\subsection{Conventional $\mathrm{O} / \mathrm{W}$ encapsulation}

The schematic of a conventional O/W encapsulation method is shown in Figure 1-8. The critical step of this method is the emulsification of a polymeric solution in an aqueous continuous phase. The $\mathrm{O} / \mathrm{W}$ emulsion is prepared by either dispersing the drug into an organic polymer solution or by mixing a drug solution with the organic polymer solution. This is followed by adding the drug-polymer mixture into an external aqueous phase containing stabilizing agents such as hydrophilic polymers under continuous agitation. Different ways have been used for the dispersion of drug and polymer mixture into the external aqueous phase. These include homogenization, microfluidizer, ${ }^{79}$ sonication ${ }^{80}$ and potentiometric dispersion. ${ }^{77}$

\subsection{O/O encapsulation}

This method is used for more hydrophobic or water insoluble drugs, whereby, the drug and polymer are dissolved in a water miscible solvent (acetonitrile). The solution is then emulsified into an oily phase in presence of emulsifier (Span 80) to form oil in oil emulsion. The organic solvent is extracted by oil and micro particles are harvested by filtration. This method is also referred to as water-in-oil (W/O) method. ${ }^{81}$

\subsection{W/O/W multiple emulsion method}

The method essentially consists of solubilizing the water soluble drugs into the primary aqueous phase, which is then emulsified into an organic solvent containing a 


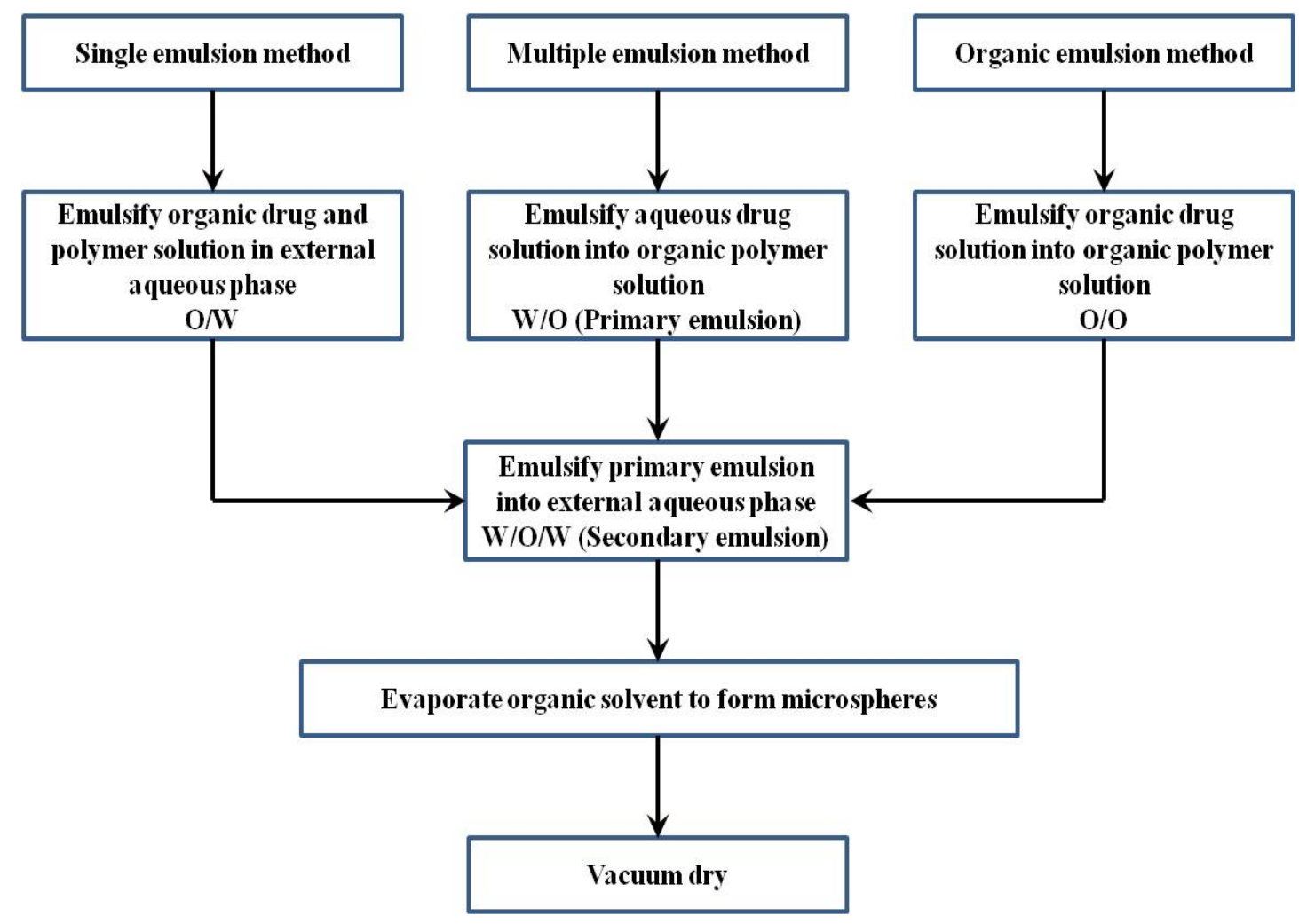

Figure 1-7. Schematic of micro particle preparation by solvent evaporation method. 


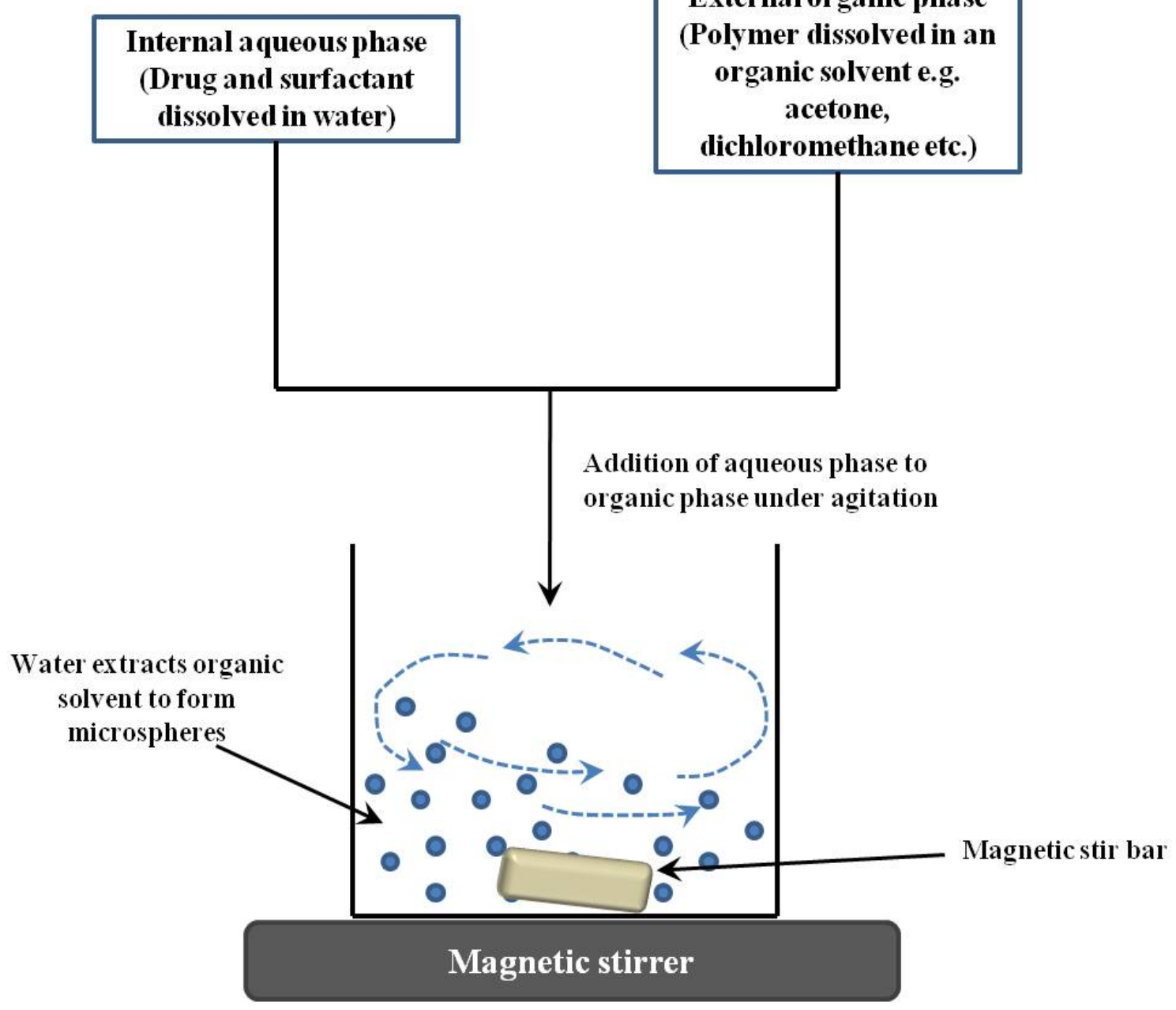

External organic phase Polymer dissolved in an acetone, dichloromethane etc.)

Figure 1-8. Schematic of an $\mathrm{O} / \mathrm{W}$ emulsion solvent evaporation method. 
biodegradable polymer. This results in the formation of a primary emulsion containing drug. The primary emulsion thus formed is then dispersed into a secondary aqueous phase under continuous agitation in order to form droplets of the primary emulsion into external aqueous phase. The organic solvent in primary emulsion is extracted out into the external aqueous phase resulting in formation of drug encapsulated polymer microspheres. The schematic of microsphere preparation by multiple emulsion method is given in Figure 1-9.

\subsubsection{Film grinding}

Polymer film grinding is yet another technique of micro particle preparation that can be used to prepare micro particles with or without the involvement of an organic solvent. PLA and PLGA micro particles have been prepared by a jet milling process by G. Nykamp et al. ${ }^{82}$ The polymer film can also be ground in a ball mill under liquid nitrogen. The particles thus obtained are usually irregular in shape unless made spherical by the impact used in jet mill and also have high percentage of surface associated drug. This surface associated drug often times leads to burst effect.

\subsection{Limitations with Biodegradable Drug Delivery Devices: Burst Effect and Tri-Phasic Release Pattern}

The biodegradable drug delivery devices discussed above have two major limitations: burst effect and tri-phasic release pattern. Most of the microsphere preparation processes result in a high percentage of surface adsorbed drug on the microspheres, which is released from the system as a huge initial burst. The implants (preformed and in situ) further release a high percentage of drug in first few hours characterized as the burst. The in situ implants utilize an organic solvent to dissolve the polymer (polymer solution based systems) and it is this solvent that carries a significant proportion of drug along with it while diffusing into the surrounding tissue after injection.

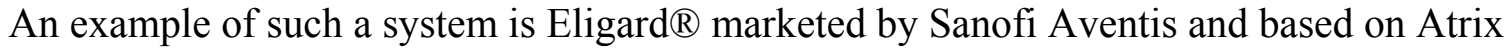
technology of in situ solvent removal and polymer precipitation. Once the solvent leaves into surrounding tissue, the polymer precipitates as a soft core in a hard shell structure. The hard shell thus formed results in a long plateau phase and a tri-phasic release profile. Figure 1-10 shows a hypothetical drug release profile from in situ PLGA based implants. The profile shows three distinct phases: burst release phase, plateau phase and erosion phase. Such a release profile is not desirable for a simple reason that the initial and the final burst phase might lead to toxic drug concentrations in the body whereas the plateau phase might result in concentrations of drug that are much below the therapeutic concentrations. The initial drug release from PLGA based in situ implants is determined by the rate of polymer precipitation upon injection. The PLGA precipitation kinetics in an in situ PLGA implant system was examined by Graham and co-workers, and Brodbeck co-workers. ${ }^{83,84}$ Parameters leading to a faster PLGA precipitation (e.g., polyvinyl pyrrolidone (PVP) or water addition to the PLGA solution or a decreasing polymer concentration) resulted in more porous implants and a high initial release. In contrast, 


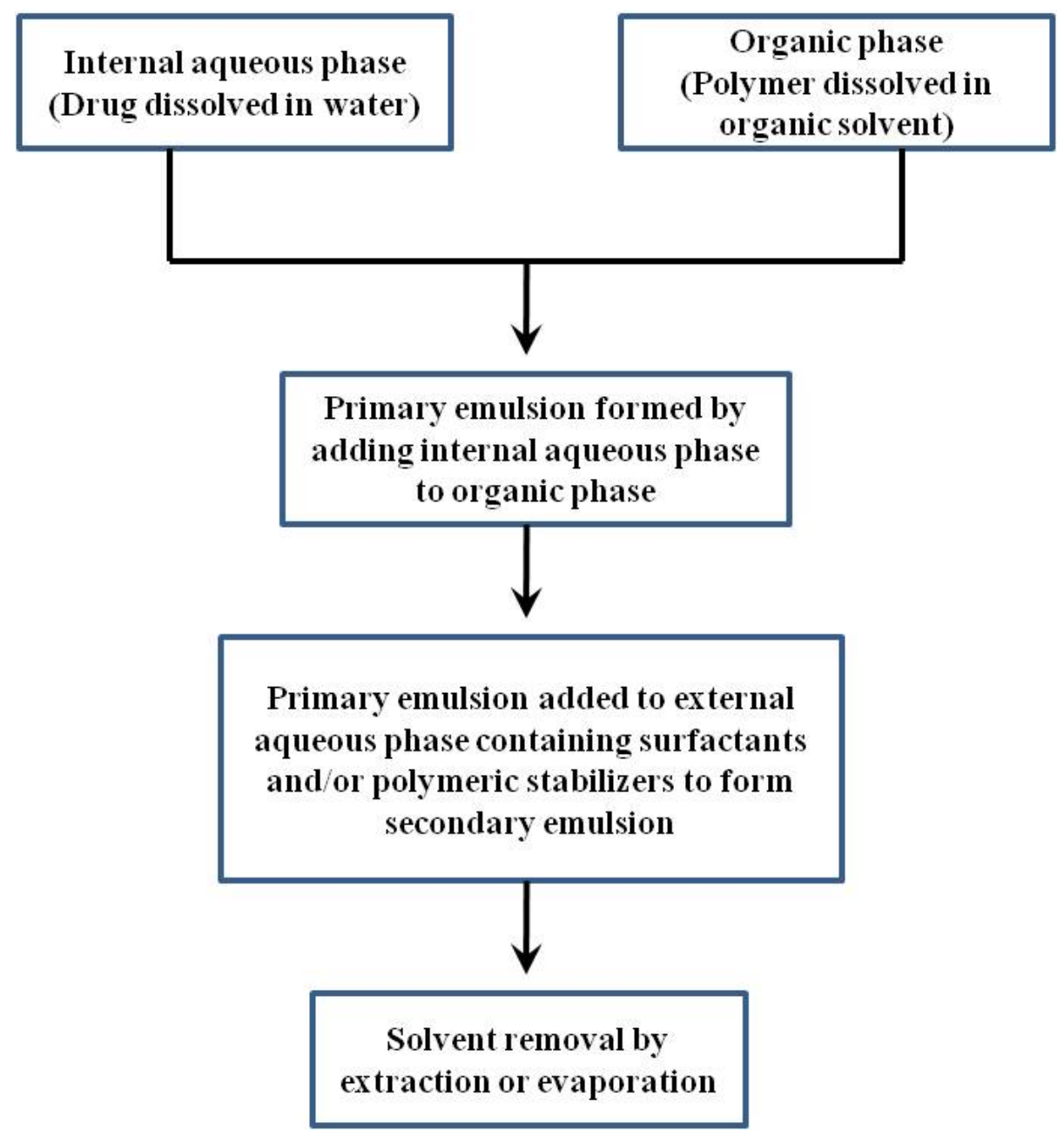

Figure 1-9. Schematic of microsphere preparation by $\mathrm{W} / \mathrm{O} / \mathrm{W}$ multiple emulsion method. 


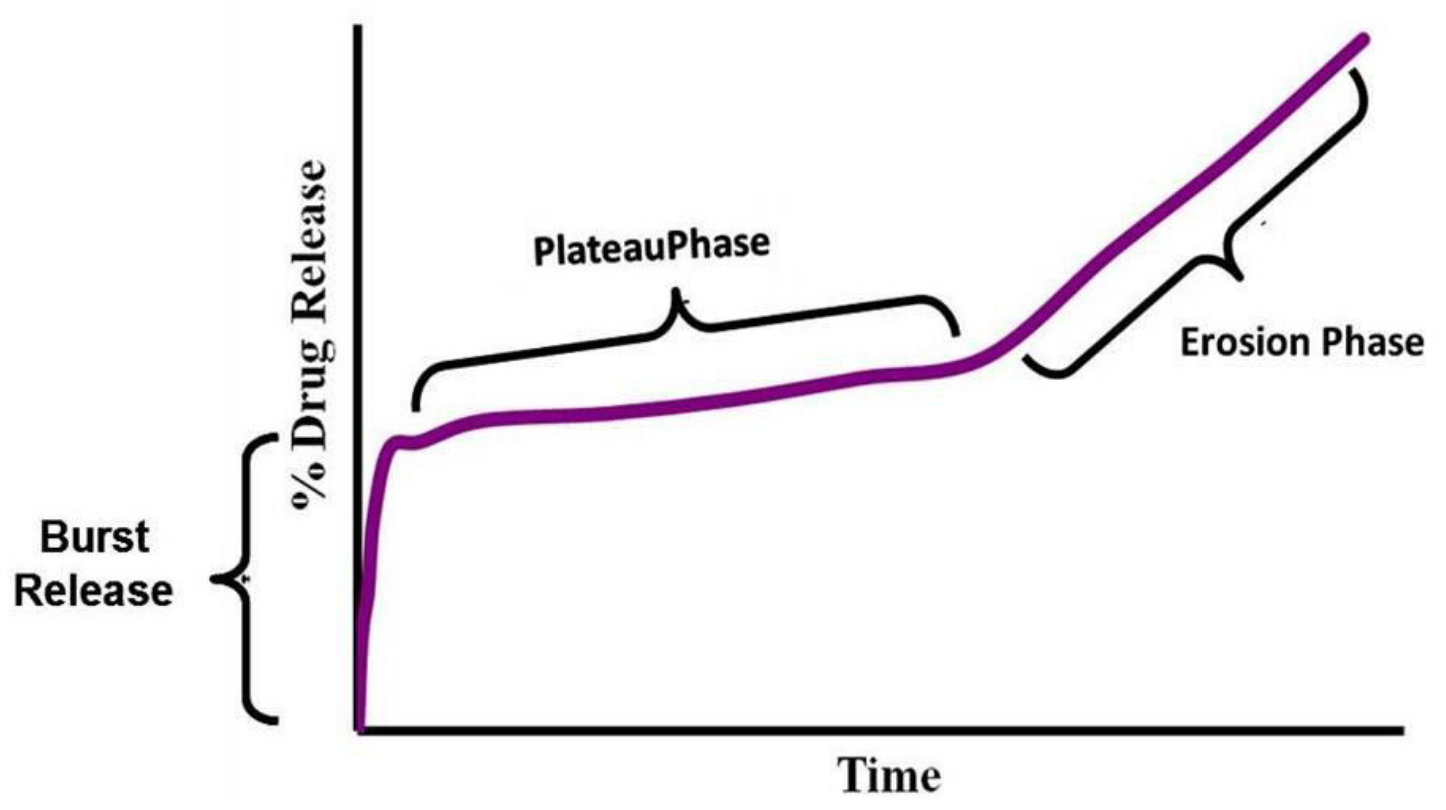

Figure 1-10. Hypothetical drug release profile from PLGA based implants. 
a slower precipitation resulted in denser sponge-like implant with a low initial release.

\subsection{In Situ Implants Using Polymer Micro Particles}

In situ gel forming polymer solution based systems have been known for a long time whereby the solvent from the polymer solution leaves out into surrounding tissue resulting in the formation of a semi solid gel like implant which hardens over time. Despite the number of applications of such systems, they have several disadvantages:

- High viscosity resulting in problematic injectability and painful injections.

- Unpredictable shape and morphology of the implant resulting in a variable solvent diffusion and therefore a variable and irreproducible drug release.

- Fast diffusion of solvent from the system resulting in a huge burst effect and eventually a tri-phasic release pattern due to polymer erosion.

- Toxicity of solvents used for the in situ precipitating systems.

To address these drawbacks, we proposed a novel suspension based in situ implant system using polymer micro particles. The idea was to cause an in situ gelling of polymer micro particles with the help of a hydrophobic polymer plasticizer resulting in formation of an implant at the site of injection by an in situ polymer gelling mechanism. This is hypothesized to address the variability in the surface area of implants thereby minimizing variability in drug release. The formulation would be administered as a micro particulate suspension having much reduced viscosity thereby overcoming the issue of painful injections. The composition of injection vehicle is quite unique so that it not only takes care of the initial burst release of drug from the formulation but also provides a relatively more continuous diffusion based drug release instead of an erosion controlled drug release. Suspension formulation would be stabilized externally after reconstitution and prior to administration by building shear thinning nature and thixotropy in the injection vehicle. Furthermore, the novel formulation was also expected to have a minimal inflammatory response due to a significant reduction in the overall amount of toxic solvents. Figure 1-11 shows the composition of the novel injection vehicle system that results in an in situ gelling of polymer micro particles.

\subsection{Objective}

The objective of this work was to develop and characterize an injectable in situ gelling polymer micro particle/ microsphere suspension system for sustained drug delivery. Specific aims include:

- To simplify the method of preparing SR depot formulations based on microspheres/ micro particles.

- To transform the tri-phasic release pattern inherent in PLGA polymer based 


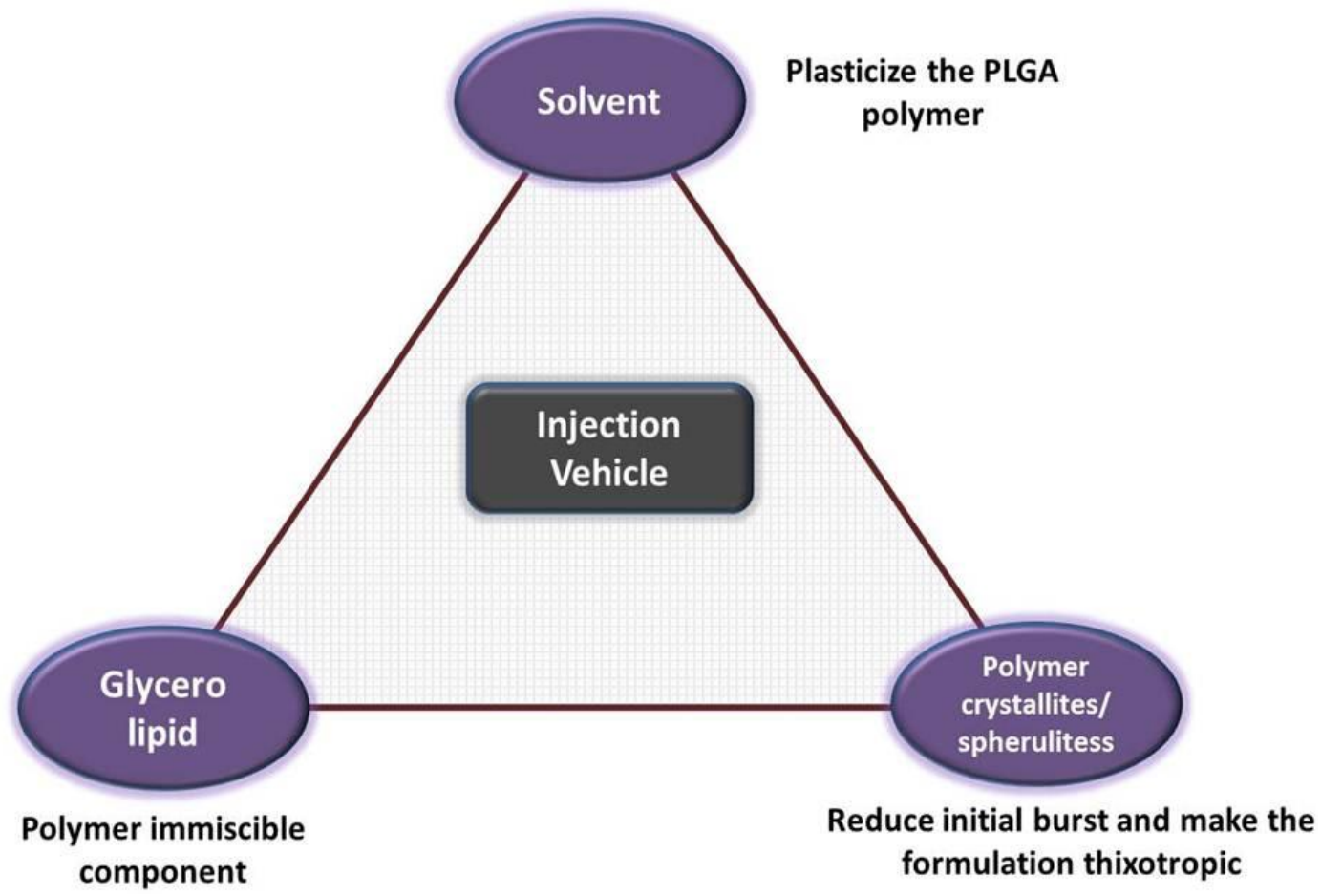

Figure 1-11. Components of the proposed injection vehicle. 
systems to a more continuous diffusion based drug release.

- To facilitate the drug administration; this would indicate mixing the formulation contents 3-4 times instead of 30-40 times.

- To reduce the variability in drug release.

- To reduce the injection viscosity and improve injectability by imparting the desired features of shear thinning and thixotropy to the formulation.

- To test the biocompatibility of injectable sustained release in situ gelling polymer micro particles based implants.

- To provide an easy to modify drug delivery system to obtain desired drug release.

\subsection{Leuprolide Acetate: Model Drug}

Leuprolide acetate is a LHRH agonist used for the treatment of prostate cancer. Prostate cancer is the most common cancer and the second leading cause of death in men in the United State ${ }^{85}$ Leuprolide acetate was first synthesized by Fujino and co-workers (Figure 1-12). ${ }^{86}$ Leuprolide works by a negative feedback mechanism. It first stimulates gonadotropin secretion by the pituitary and steroidogenesis in the genital organs. On long term continuous administration, however, the excess of testosterone thus produced exerts an antagonistic effect on the pituitary and stops any further production of the leutinizing hormones. $^{87}$

Leuprolide is a nonapeptide (MW $1209 \mathrm{Da}$ ) with very poor oral absorption properties. It has limited bioavailability through other administration routes such as transdermal ${ }^{87}$ nasal,${ }^{88}$ and vaginal. ${ }^{89}$ Leuprolide was first marketed as a daily subcutaneous injection ${ }^{90}$ and was later released as several parenteral controlled release products including Viadur ${ }^{\mathrm{TM}}$, Lupron Depot ${ }^{\circledR}$, and Eligard ${ }^{\circledR}$ to provide patient compliance and reduce the frequency of administration.

\subsubsection{Viadur ${ }^{\mathrm{TM}}$}

Viadur ${ }^{\mathrm{TM}}$ is a testosterone suppression therapy for the palliative treatment of advanced prostate cancer. In an in-office procedure, the product is implanted under the skin under the upper arm of the patient, and using the DUROS ${ }^{\circledR}$ implant technology, Viadur $^{\mathrm{TM}}$ delivers continuous, osmotically-driven treatment for an entire year. The product delivers 12 months of testosterone suppression therapy in a single administration. The implant consists of a cylindrical titanium alloy reservoir capped on one end by a rate controlling membrane and at the other end by a diffusion moderator containing an orifice through which drug is released from the system. Although long term drug delivery is a definite advantage of this system, the insertion and surgical removal of this implant are painful procedures. Therefore patient compliance is an issue with such systems that are meant for surgical implantation. 


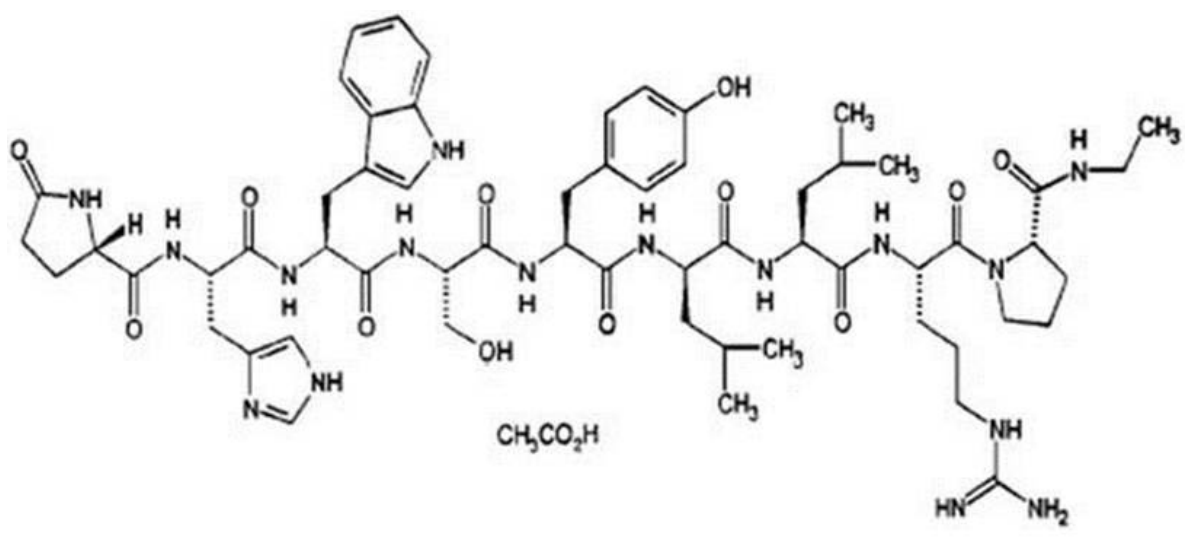

S-oxo-Pro-His-Trp-Ser-Tyr-D-Leu-Leu-Arg-Pro-NH- ${ }_{2} \mathrm{H}_{5}$

Leuprolide

Figure 1-12. Amino acid sequence of leuprolide acetate. 


\subsubsection{Lupron ${ }^{\circledR} \operatorname{depot}$}

Lupron ${ }^{\circledR}$ depot consists of PLGA microspheres prepared by a $\mathrm{W} / \mathrm{O} / \mathrm{W}$ multiple emulsion method encapsulating leuprolide acetate. The system has been marketed for 1, 3 and 4 month drug delivery. The microspheres are administered as a suspension in water for injection and have to be administered immediately after suspension formation to prevent settling of microspheres in the syringe. The microspheres provide a huge burst release of drug and involve a multiple and complex manufacturing process.

\subsubsection{Eligard $®$}

Eligard ${ }^{\circledR}$ is an injectable in situ forming implant formulated as a polymer solution that releases the solvent upon administration. This results in in situ polymer precipitation according to the Atrix technology. ${ }^{27,91}$ The system is marketed as a two syringe system, whereby one of the syringe contains drug and second syringe contains PLGA solution in NMP. This system avoids the complex preparation of the formulation and thus reduces the investment and cost; however, they also have some limitations. The high viscosity of the PLGA solution may lead to a painful injection; the surface area of the resulting implant, controlling the drug release, may be variable depending on the injection technique and site; in addition, a high initial release may occur because of the formation of highly porous implants. 


\section{CHAPTER 2. SELECTION OF DISSOLUTION METHODOLOGY}

\subsection{Introduction}

There is an increase in the number of novel dosage forms developed in past few years such as controlled release parenteral microspheres, liposomes and implants. Currently, many of the protein and peptide drugs are being formulated as polymer microspheres for parenteral administration. These include leuprolide, goserelin, buserelin, triptorelin, human growth hormone, and octreotide etc. Apart from the peptide and protein drugs there are quite a few small molecules formulated as polymer microspheres for parenteral delivery i.e. risperidone, doxitaxel etc. Dissolution testing has become increasingly important in quality control and formulation development as a tool for the assessment of the in vivo behavior. ${ }^{92}$ Furthermore, in vitro dissolution testing has also been recommended by the food and drug administration (FDA) Generic Advisory Committee as a surrogate marker for bioavailability and bioequivalence. ${ }^{93}$ In vitro testing of dosage forms is not only necessary to develop and in vitro-in vivo correlation (IVIVC), but also is required as a discriminatory method to test differences between various formulations. This requires the in vitro testing method to have enough discriminatory power in order to distinguish between good and bad a formulation, which is then essential to investigate batch to batch variability between formulations, effect of raw materials from different manufacturing lots and the process variables. Despite the importance of in vitro testing in order to predict the in vivo behavior of drugs, none of the official dissolution techniques known are currently approved for the parenteral microsphere systems. Standard methods used currently for dissolution testing were originally developed for oral and transdermal drug products rather than parenteral delivery systems. These methods cannot be used for the parenteral products as they generally require large volume of dissolution media. The dynamics of a parenteral injection site are significantly different from those of the GI Tract and considering the comparatively smaller amount of tissue fluid and low blood flow at these injection sites, a different dissolution system is needed for parenteral products. The methods currently used for the release testing of injectable drug products include mainly membrane diffusion, sample and separate, in situ and continuous flow methods. ${ }^{94}$ The more conventional method is the sample and separate method, often referred to as the tube method, in which drug-loaded microspheres are introduced into a sealed tube or vial or a stoppered Erlenmeyer flask containing buffer, and release is followed over a specified time. ${ }^{95-100}$ Advantages of the sample and separate method are accurate measurement of the initial burst of drug from microspheres and maintenance of sink conditions by replacement of the buffer. ${ }^{94}$

Two major criteria for the development of an appropriate in vitro dissolution method are the apparatus selection and the physiological factors at the subcutaneous implantation site. Important factors involved in dissolution apparatus selection are as follows. ${ }^{101}$

- Simple design

- Convenience in handling, operation and cleaning 
- Well-defined components for reproducible results

- Provision for an easy introduction of the test product and sample withdrawal for analysis

- 'Bio relevant' to the extent feasible, i.e. should mimic physiological condition at the site of implantation

- Allow effective and controlled agitation

- Potential for use in accelerated drug release tests

- Economical

Figure 2-1 shows the subcutaneous tissue environment around an implant. The implant's outer surface is in immediate contact with the interstitial tissue space from where the drug eventually diffuses into the blood stream. The major problem with most of the parenteral depot formulations is their non-defined shape and volume post injection which makes it difficult to test these products in a similar manner to oral drug products. Therefore, it becomes necessary to find a system where the formulation can be trapped in one part of the dissolution apparatus and there is no instance of the formulation being disturbed during sampling procedures. One way of trapping the formulation is to place it inside a closed mesh cloth. However, one has to be very careful about the unhindered diffusion of drug from the cloth as it might retard the drug diffusion further and give us a false impression of drug release. Another such method is the use of dialysis bags, that can be used to trap the formulation in contact with a small volume of dissolution buffer and the dialysis tube is then placed inside the larger buffer volume. Such type of in vitro dissolution method has been used by some researchers, not only to study the long term release from the formulation but also as a device to test the release under accelerated conditions. ${ }^{94}$ However, the major limitation with this methodology is expected to be a lag in the drug diffusion process as the drug first equilibrates with the surroundings inside the dialysis tube or bag before it eventually starts to diffuse out into the larger volume of dissolution media. The actual drug release during the first few time points is therefore not captured properly with this method.

Therefore the objective of this study was to identify the most appropriate method for in vitro testing of the formulations developed in the present work by comparing four different dissolution techniques i.e. nylon cloth mesh, dialysis bag, teflon cavity method and sample and separate method.

\subsection{Materials and Methods}

\subsubsection{Materials}

The controlled release polymer i.e. PLGA $(0.24$ and $0.59 \mathrm{dL} / \mathrm{g})$ was procured from Lactel absorbable polymers, Durect Corporation, Pelham, AL, USA. Other 


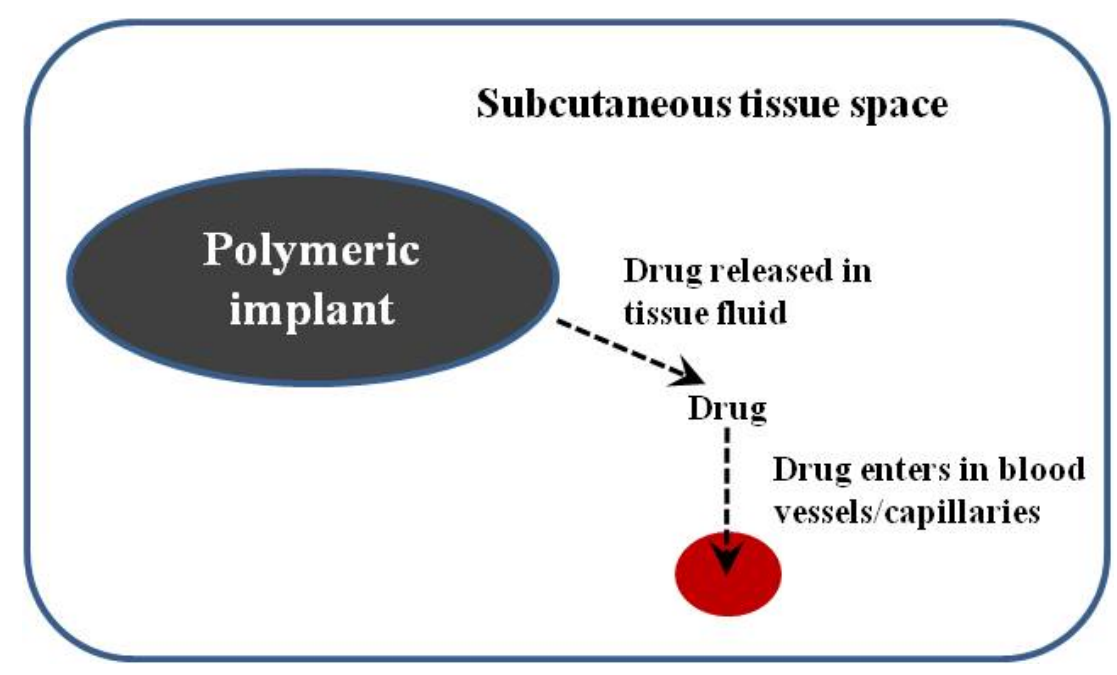

Figure 2-1. Visualization of subcutaneous tissue environment around implant. 
solvents/plasticizers i.e. triethyl citrate (TEC) and acetyl triethyl citrate (ATEC) were obtained from Morflex Inc., Greensboro, NC, USA and NMP (N-methyl pyrrolidone; Pharmasolve) was procured from ISP Pharma Technologies, Wayne, NJ, USA. Leuprolide was procured from Teva Ltd., Israel). Glyceryl monooleate (GMO) was received as a gift sample from Abitec Corp., Janesville, WI, USA. Float-A-Lyzer (25 kilodalton (KD) molecular weight cut off (MWCO), 3-mL capacity, regenerated cellulose membrane) was purchased from Spectrum Labs, Rancho Dominguez, CA. Nylon mesh (10 $\mu \mathrm{m}$ opening $28 \mu \mathrm{m}$ thread diameter $2 \%$ open area) was purchased from Amazon.

\subsubsection{Methods}

\subsubsection{Analytical method development}

An appropriate amount of leuprolide was dissolved in reverse osmosis (RO) water to obtain a stock solution of $1000 \mathrm{ug} / \mathrm{ml}$ concentration. Appropriate aliquots were withdrawn and diluted to $10 \mathrm{ml}$ each in order to obtain drug standards at various concentration levels ( $5 \mathrm{ug} / \mathrm{ml}$ to $50 \mathrm{ug} / \mathrm{ml}$ ). Leuprolide Acetate was analyzed by reverse phase (RP)-HPLC using a C18 column, $150 \mathrm{~mm} \times 4.60 \mathrm{~mm}$ (Phenomenex, Torrance, $\mathrm{CA})$. A gradient elution method was used with mobile phase A $(0.1 \% \mathrm{v} / \mathrm{v}$ trifluoroacetic acid in water) and mobile phase $\mathrm{B}(0.1 \% \mathrm{v} / \mathrm{v}$ trifluoroacetic acid in acetonitrile). The gradient was set as $10 \%$ to $20 \%$ solvent B during first $2.5 \mathrm{~min}, 20 \%$ to $27 \%$ solvent B from 2.5 to $6 \mathrm{~min}, 27 \%$ to $60 \%$ solvent B from 6 to $8 \mathrm{~min}$, a constant flow of $60 \%$ solvent $\mathrm{B}$ from 8 to $15 \mathrm{~min}$ and $60 \%$ to $10 \%$ solvent B from 15 to $20 \mathrm{~min}$. The flow rate was set at $1.5 \mathrm{~mL} / \mathrm{min}$ and UV absorbance was measured at $215 \mathrm{~nm}$ using a photodiode array detector equipped with the high performance liquid chromatography system (HPLC). Standard curve was plotted at the aforementioned concentration range resulting in a good linearity value (regression coefficient, $\mathrm{R}^{2}=0.9998$ ).

\subsubsection{Preparation of micro particles}

Polymer micro particles were prepared by a film grinding method whereby the polymer was first dissolved in a solvent i.e. acetone. Once the polymer solution was formed, drug powder was dispersed in it while the entire system was still under magnetic stirring. The dispersion was allowed to stir for about $30 \mathrm{~min}$ in order to completely break the drug agglomerates into fine particles and covered with an aluminum foil so as not to allow any solvent to evaporate during this time. The dispersion was then left open in a fume hood while under stirring and the solvent was allowed to evaporate. Once a significant amount of solvent was evaporated from the dispersion, the partially dried drug-polymer mass thus obtained with a semi-solid consistency was then transferred to a Teflon petri dish and left in the hood for drying at room temperature for about 24 hours. The flexible film was then kept in a vacuum oven for next 24 hours in order to allow evaporation of the remaining solvent. The oven was set to a temperature of $50^{\circ} \mathrm{C}$ and 25 in $\mathrm{Hg}$ of vacuum. The dried film was then ground into micro particles by using a cryo 
mill. Briefly, the milling procedure involved first placing the film in the cryo mill jar which was then immersed in the liquid nitrogen for 10 minutes. Once the liquid nitrogen stops bubbling, the jar was taken out and fixed in place in the mill. The mill was then operated for about 4 minutes at the frequency of $28 \mathrm{~Hz}$. The schematic of micro particle preparation process is depicted in Figure 2-2.

\subsubsection{Preparation of formulation}

The formulation for the dissolution studies was prepared by packing the drug-polymer micro particles equivalent to $7.5 \mathrm{mg}$ of the leuprolide dose for one month in a $3 \mathrm{ml}$ plastic syringe and filling the other syringe with 800 microlitres of the carrier/vehicle system. Formulations used for testing under different dissolution settings are listed in Table 2-1. The contents of both syringes were mixed with each other a 3 to 5 times to prepare a suspension which was then placed in the individual dissolution set ups. The simulated marketed formulation (Eligard ${ }^{\circledR}$ ) was also tested with various dissolution set ups in a similar manner after mixing the content of the formulation.

\subsubsection{Dissolution test conditions}

Drug release studies were conducted in $10 \mathrm{ml}$ of dissolution media, which was $0.012 \mathrm{M}$ phosphate buffer saline (PBS) containing $0.02 \%$ sodium azide. The buffer was

prepared as per the composition in Table 2-2. For study involving nylon cloth mesh, small bags were made out from a circular piece of cloth. The cloth was then wet with the dissolution media and formulation was placed inside the bag, which was then sealed tightly with a nylon thread and placed inside the vial containing the dissolution media. For the dialysis bag study, the solution inside the bags was first discarded after which the dialysis membrane was soaked in the dissolution media for 30 minutes. The formulation was then injected inside the dialysis bags along with $2 \mathrm{ml}$ of dissolution media and the entire set up was placed inside a container having larger volume $(10 \mathrm{ml})$ of dissolution media. Samples (2 ml) were withdrawn at 1, 3, 5, 10 and 24 hours and at 2, 4, 8, 12, 16, 20,25 and 30 days. The aliquots withdrawn were immediately replaced by fresh buffer maintained at $37^{\circ} \mathrm{C}$. The samples were analyzed by the RP-HPLC method.

\subsubsection{IVIVC development for Eligard ${ }^{\circledR}$ formulation}

A two compartment first order elimination model (macro constants) was selected in WinNonlin version 5.2 according to the scheme shown in Figure 2-3. Plasma leuprolide concentration-time profiles following administration of IV bolus leuprolide solution were fitted by the aforementioned model in order to derive the values of macro constants i.e. A, B, $\alpha$ and $\beta$. Goodness of fit was evaluated by inspection of residual errors and the Akaike information criteria (AIC). The macro constants thus obtained were provided as the input values for the deconvolution wizard in WinNonlin. The in vivo release values thus obtained were interpolated to achieve the same time scale for 


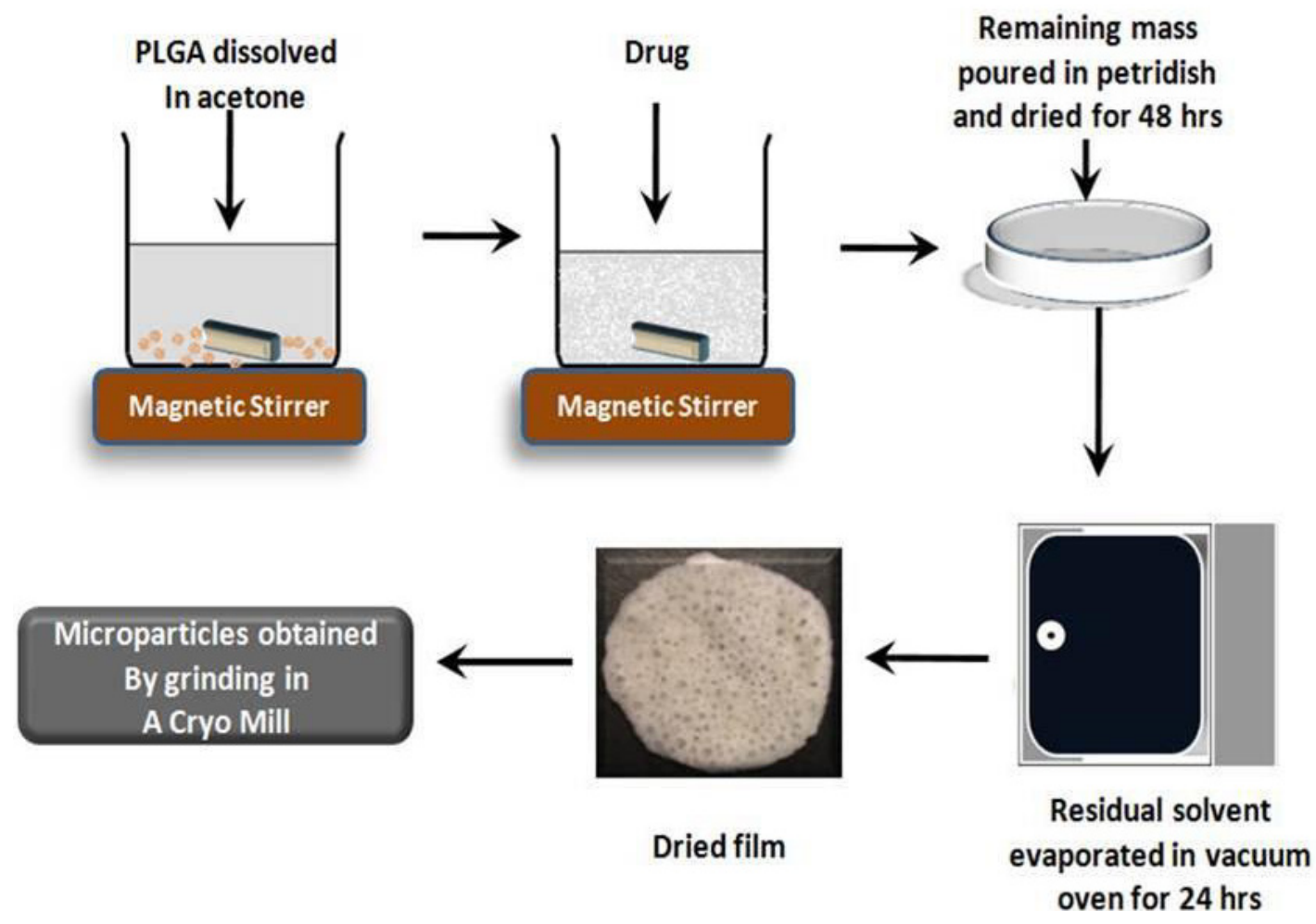

Figure 2-2. Schematic representation of PLGA micro particle preparation by film grinding. 
Table 2-1. Formulations used for testing under different dissolution set-ups.

\begin{tabular}{lccc}
\hline S. No. & $\begin{array}{c}\text { Drug loading } \\
\text { (\% w/w of } \\
\text { polymer) }\end{array}$ & $\begin{array}{c}\text { PLGA } \\
\text { intrinsic } \\
\text { viscosity } \\
\text { (dL/g) }\end{array}$ & Vehicle/Carrier \\
\hline & & & \\
1 & 4 & 0.24 & ATEC:NMP (9:1):GMO (1:5) \\
2 & 4 & 0.59 & ATEC \\
3 & 4 & 0.59 & ATEC:NMP (9:1):GMO (1:5) \\
4 & 8 & 0.24 & ATEC:NMP (9:1):GMO (1:5) \\
5 & 16 & 0.59 & ATEC:NMP (9:1):GMO (1:5) \\
\hline
\end{tabular}

Table 2-2. Buffer composition for dissolution medium.

\begin{tabular}{lc}
\hline Buffer components & Amount (g/l) \\
\hline & \\
Sodium chloride & 8.0 \\
Potassium chloride & 0.2 \\
Disodium hydrogen phosphate & 1.44 \\
Potassium dihydrogen phosphate & 0.24 \\
Sodium azide & 0.2 \\
\hline
\end{tabular}

Note: Ionic strength of the buffer was calculated to be $0.16 \mathrm{M}$. 


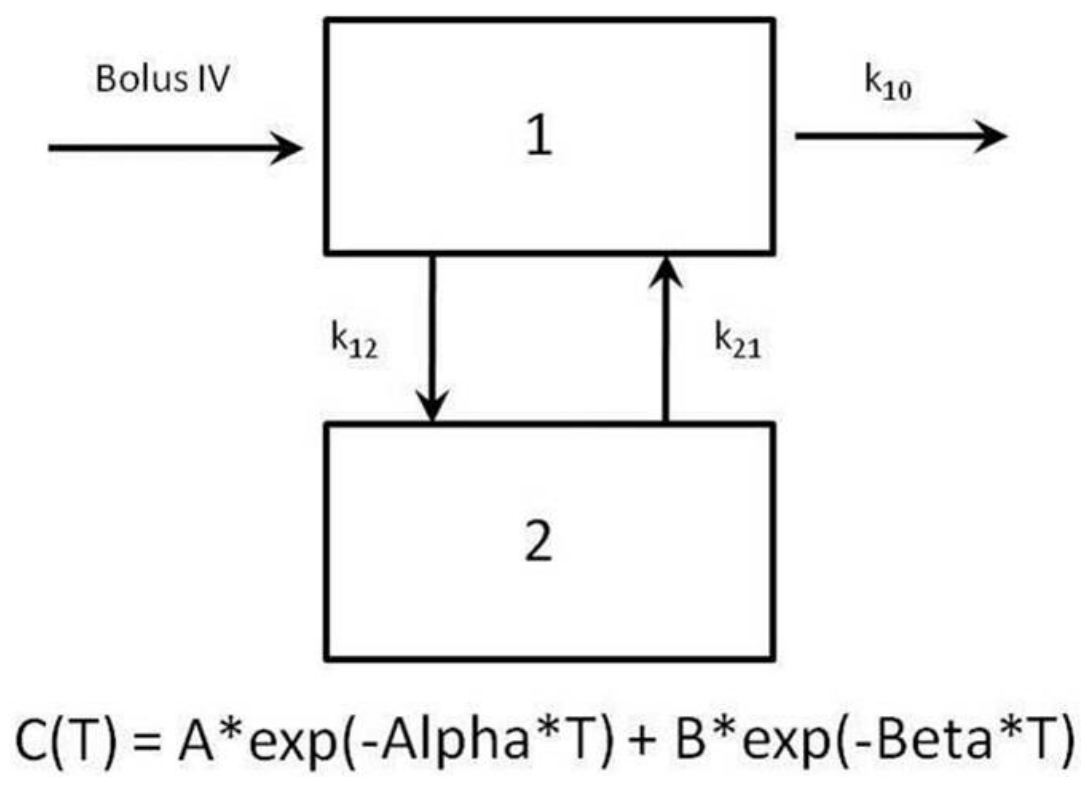

Figure 2-3. Pharmacokinetic model used for fitting of plasma leuprolide concentration data following administration of IV bolus.

Note: A and alpha $(\alpha)$ are the macro constants for the distribution phase and B and beta $(\beta)$ are macro constants for elimination phase. 
both in vivo and in vitro drug release. A two piece and three piece regression models were compared in terms of describing the relationship between in vitro and in vivo drug release by using Sigma Plot.

\subsection{Results and Discussions}

\subsubsection{Analytical method development}

Figure 2-4 shows the standard curve for leuprolide with linearity $\left(\mathrm{R}^{2}\right)$ of 0.9998 in the concentration range of $5 \mu \mathrm{g} / \mathrm{ml}$ to $50 \mu \mathrm{g} / \mathrm{ml}$. Method validation results including intraday and interday variation and accuracy are given in Table 2-3.

\subsubsection{Drug release studies: in vitro release set ups}

Figure 2-5, 2-6, 2-7 and 2-8 shows the pictorial representation of the four different types of in vitro drug release experimental set ups. The sample and separate method essentially consisted of the solidified or gelled formulation placed at the bottom of the glass vial or glass bottle (Figure 2-5). The formulation was exposed to the dissolution media from the top surface and the sides. The surface at the bottom of the formulation was not in direct contact with the dissolution media but it accounted for only a small percentage of the overall surface area of the formulation.

The dialysis set up utilized a Float-A-Lyzer that essentially consisted of a dialysis membrane folded as a tube and a pre-sealed base on one end of the tube (Figure 2-6). The formulation was placed inside the dialysis tube, whereby the formulation resided at the bottom of the tube and the remaining space inside the tube was filled with dissolution buffer. The drug was released mainly from the topmost surface of the formulation and it equilibrated with the dissolution media inside the tube before diffusing into the outer media compartment. Effectively, there are two steps involved before the appearance of drug in the outer compartment that are responsible for the observed lag time in drug release: equilibration of drug between the inner and outer compartment and saturation of dialysis membrane with the drug.

The nylon mesh method of in vitro drug release studies included the formulation contained inside a nylon pouch, which was placed inside the vial containing dissolution media (Figure 2-7). The pouch was first allowed to wet completely with the dissolution media separately before placing the formulation inside. The pouch was attached to the top of dissolution vessel with the help of a nylon thread such that the formulation was exposed to dissolution media from all the surfaces. The Teflon cavity method consisted of a cylindrical cup shaped cavity carved out in Teflon and a Teflon lid to cover the Teflon cavity (Figure 2-8). The lid further contained a stainless steel mesh (size equal to US sieve \# 120) which allowed the diffusion of dissolved drug from the formulation. In this set up, only the top surface of formulation was exposed to the dissolution media. 


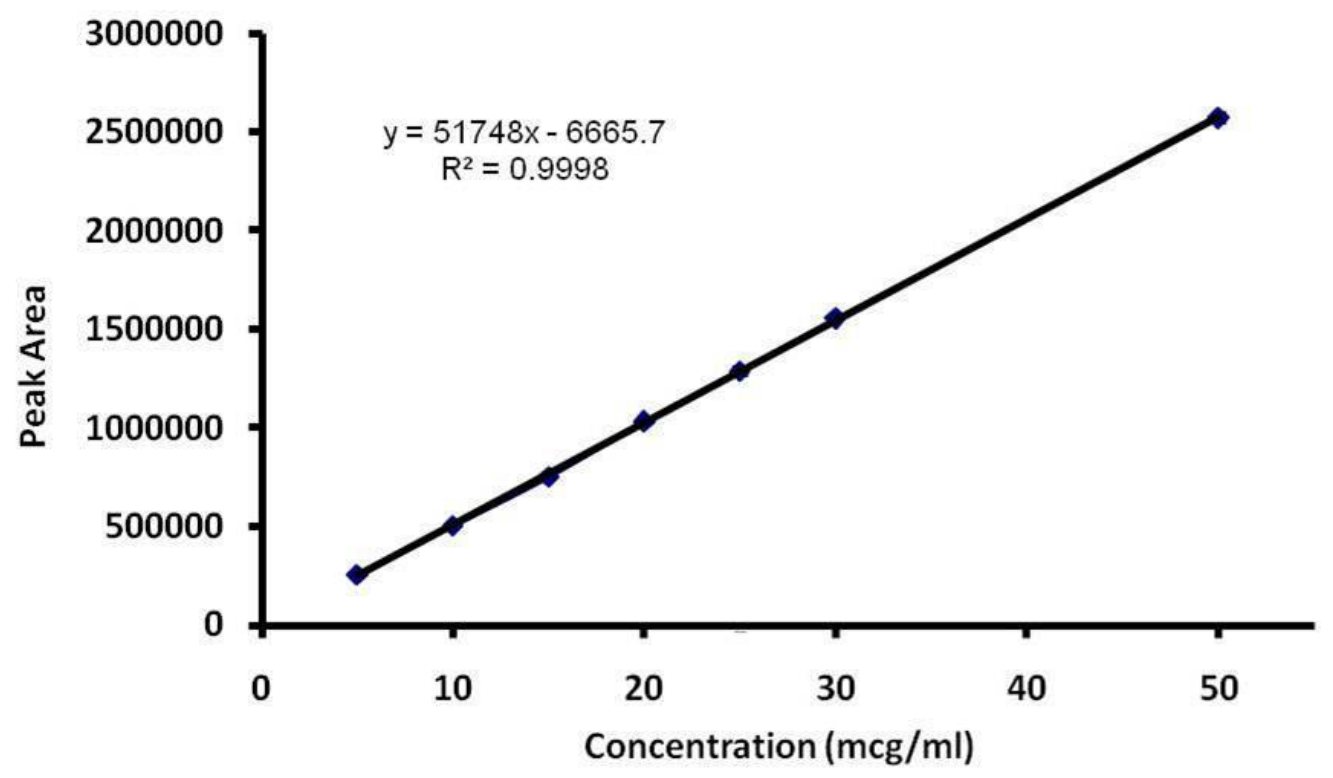

Figure 2-4. HPLC standard curve for leuprolide.

Table 2-3. Intraday and interday variability for leuprolide HPLC assay.

\begin{tabular}{lcc}
\hline $\begin{array}{l}\text { Drug concentration } \\
\text { (ug/ml) }\end{array}$ & $\begin{array}{c}\text { Intraday \% } \\
\text { RSD }\end{array}$ & $\begin{array}{c}\text { Interday \% } \\
\text { RSD }\end{array}$ \\
\hline & & \\
5 & 1.36 & 1.32 \\
10 & 0.94 & 1.2 \\
15 & 1.38 & 1.24 \\
20 & 0.45 & 0.42 \\
25 & 1.60 & 0.98 \\
30 & 0.56 & 0.75 \\
50 & 0.94 & 1.23 \\
100 & 1.06 & 1.31 \\
150 & 0.48 & 0.87 \\
200 & 0.48 & 0.74 \\
\hline
\end{tabular}

Note: RSD is the relative standard deviation also known as percent coefficient of variation. 


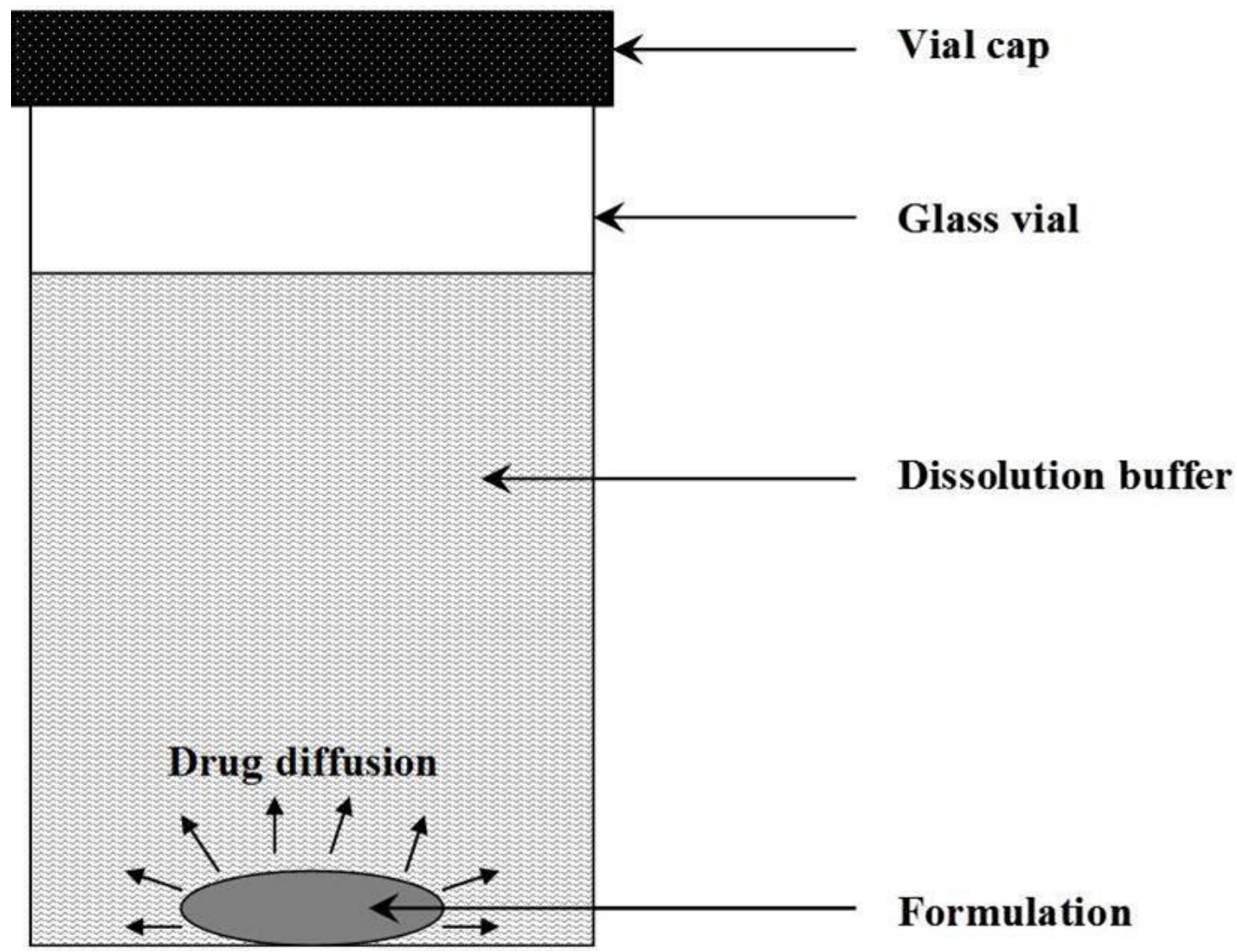

Figure 2-5. Illustration of set up for in vitro drug release from PLGA formulations using a sample and separate method. 


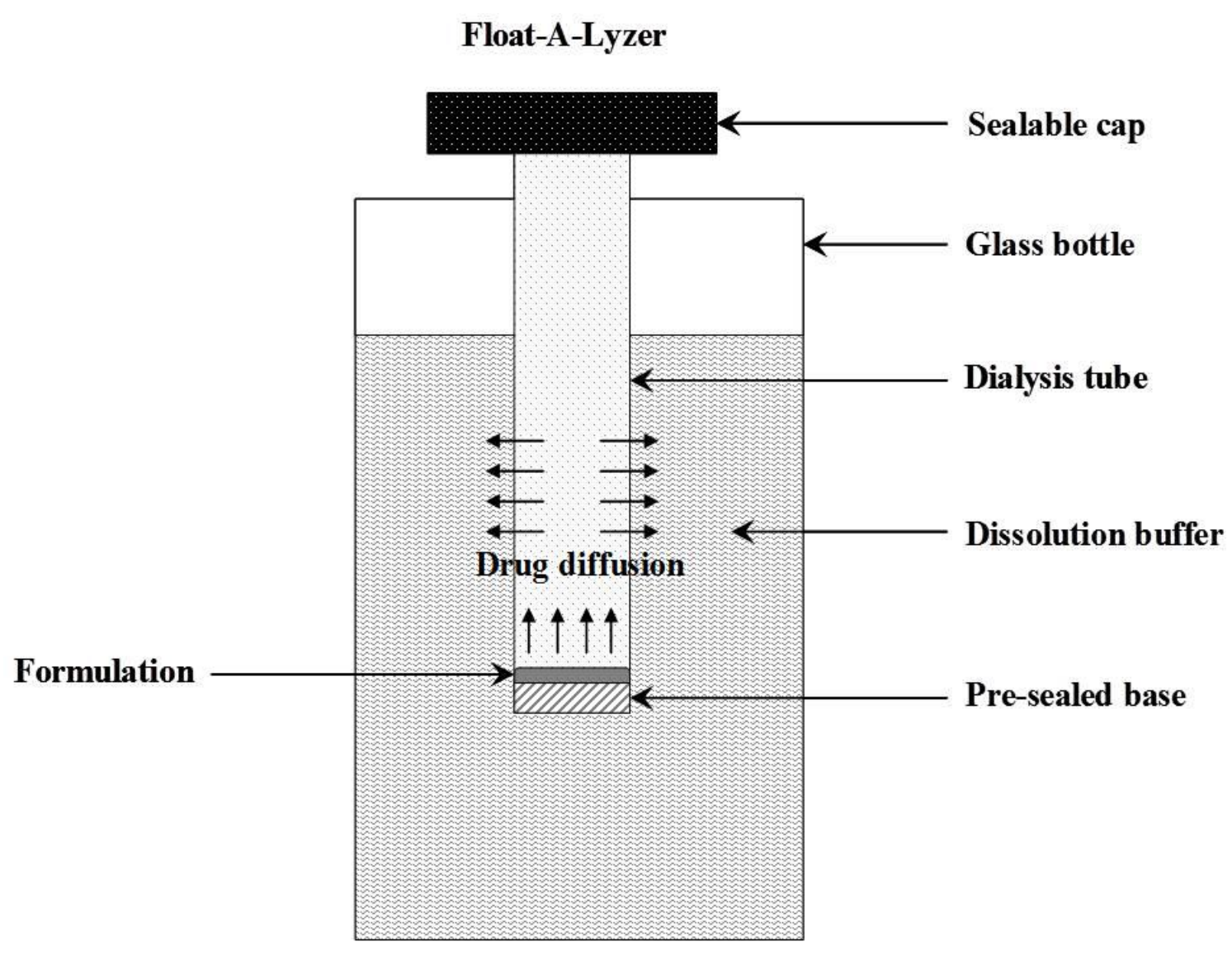

Figure 2-6. Illustration of set up for in vitro drug release from PLGA formulations using a Float-A-Lyzer. 


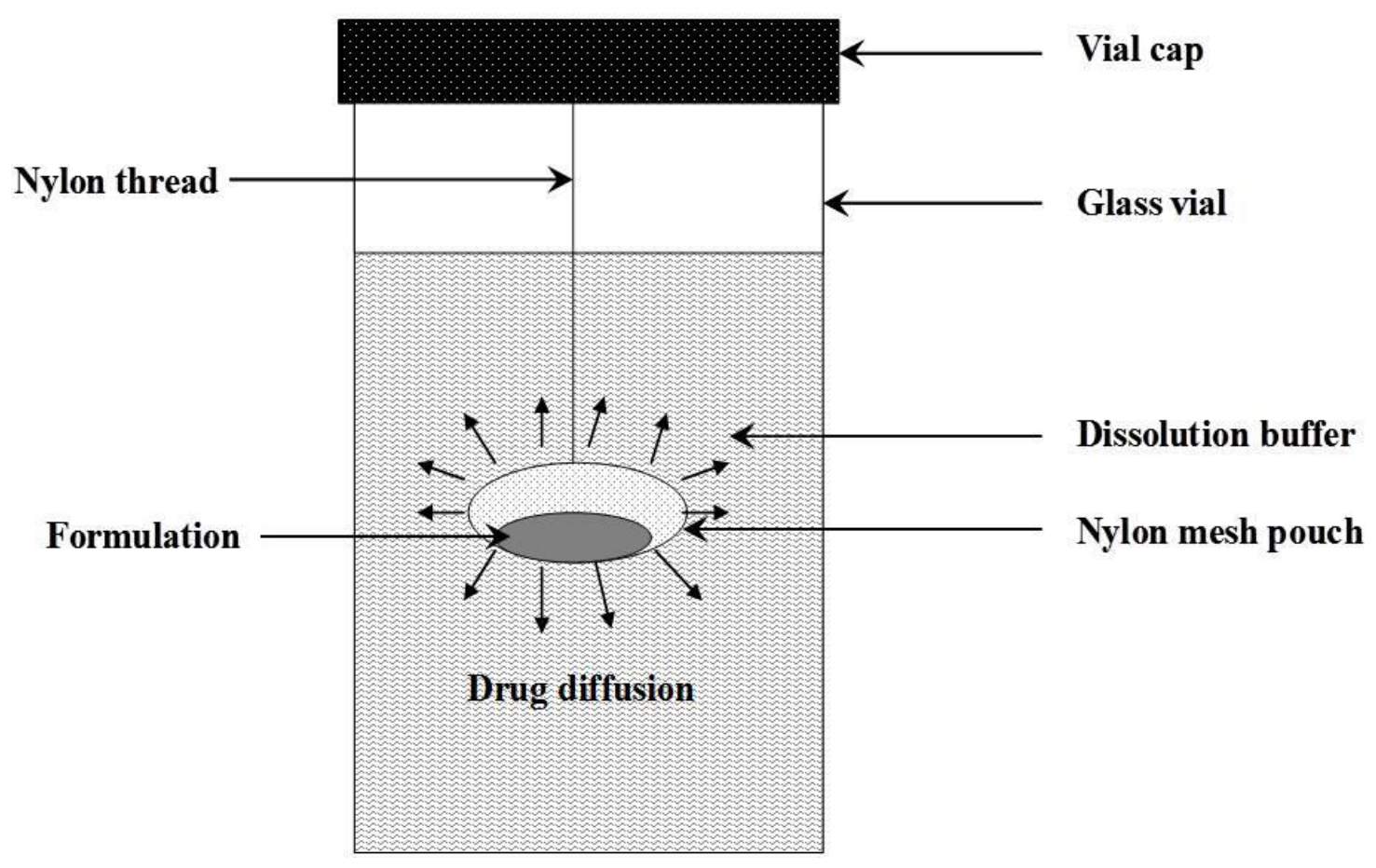

Figure 2-7. Illustration of set up for in vitro drug release from PLGA formulations using a nylon membrane pouch. 


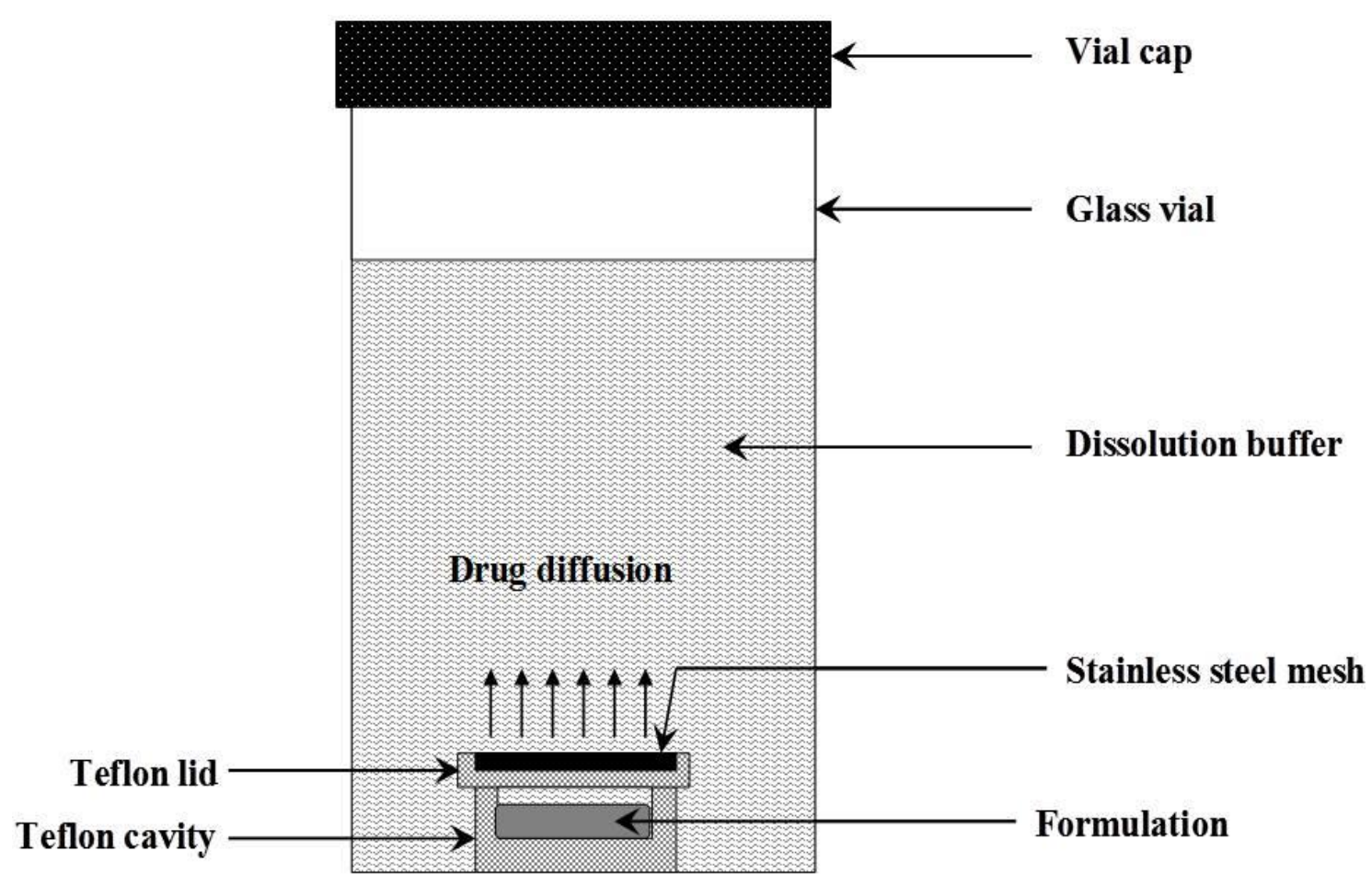

Figure 2-8. Illustration of set up for in vitro drug release from PLGA formulations using a Teflon cavity with lid. 


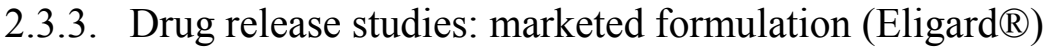

The simulated marketed formulation showed a significant difference in the in vitro drug release between four dissolution set ups. The calculated $\mathrm{f}_{2}$ (similarity factor) values were less than 50 for comparison between most of the set ups which indicated a significant difference between the drug release profiles (Table 2-4). The in vitro release profiles thus obtained from different release set ups were further compared with the deconvoluted plasma profile of the simulated Eligard $\AA$ formulation (Figure 2-9). Three distinct phases could be identified in the deconvoluted profile i.e. initial burst release phase where about $72 \%$ drug was released within first 24 hours, plateau phase that lasted for 4.5 days where only a minimal drug was released and final erosion phase where the remaining drug was released much faster as a second burst. An ideal in vitro release method should also demonstrate all the three phases to be able to mimic the in vivo drug release. Only two of these phases i.e. initial burst release phase and the second plateau phase could be visualized with the nylon mesh and Teflon cavity in vitro set ups. The erosion phase was missing within the time frame of 30 days and could have appeared after 30 days. Although dialysis method exhibited the three phases, there was a significant difference in overall cumulative drug release when compared with the deconvoluted plasma profile. The sample and separate method on the other hand, exhibited all the three phases quite distinctly with a relatively smaller difference in overall cumulative drug release when compared with the deconvoluted plasma profile.

Further, the cumulative in vitro drug release from the four different methods were compared and correlated with the in vivo release (obtained from deconvoluted profile) in order to find the predictability of in vivo release from the in vitro data. A piecewise regression model representing 3-piece regression was used to build the correlation between in vitro and in vivo drug release, whereby the 3 different regression pieces represented the three drug release phases. Table 2-5 shows the $\mathrm{R}^{2}$ values obtained by modeling the correlation between in vitro and in vivo drug release by a 3-piece regression model. The correlation between drug release by the sample and separate method and the in vivo drug release was the best with the highest $\mathrm{R}^{2}$ value $(0.991)$. This provides a validity of this in vitro method for release testing of PLGA based depot formulations compared to other methods i.e. dialysis, nylon mesh and Teflon cavity. A 2-piece regression model was also used to validate the results and to confirm the absence of correlation between in vitro and in vivo drug release for the other methods. The $\mathrm{R}^{2}$ values obtained for the 2-piece regression model were even lower thus confirming the validity of the 3-piece regression model to build correlation between the in vitro and in vivo drug release (Table 2-5). Table 2-6 summarizes the prediction errors obtained for various in vitro methods using the 3-piece regression model. The sample and separate method demonstrated least percentage of prediction error among different in vitro test methods.

\subsubsection{Drug release studies: in house preliminary formulations}

To find the validity of above observation (market formulation) in other PLGA based formulations, a similar comparison was done between various in vitro test methods 
Table 2-4. $\quad \mathrm{f}_{2}$ values for comparison between various in vitro test methods.

\begin{tabular}{lcc}
\hline In vitro release set up (1) & In vitro release set up (2) & $\mathbf{f}_{2}$ value \\
\hline & & \\
Sample and separate & Float-A-Lyzer & 26.6359 \\
Sample and separate & Nylon mesh & 35.9114 \\
Sample and separate & Teflon cavity & 32.6258 \\
Float-A-Lyzer & Nylon mesh & 39.2638 \\
Float-A-Lyzer & Teflon cavity & 44.8798 \\
Nylon mesh & Teflon cavity & 63.2714 \\
\hline
\end{tabular}

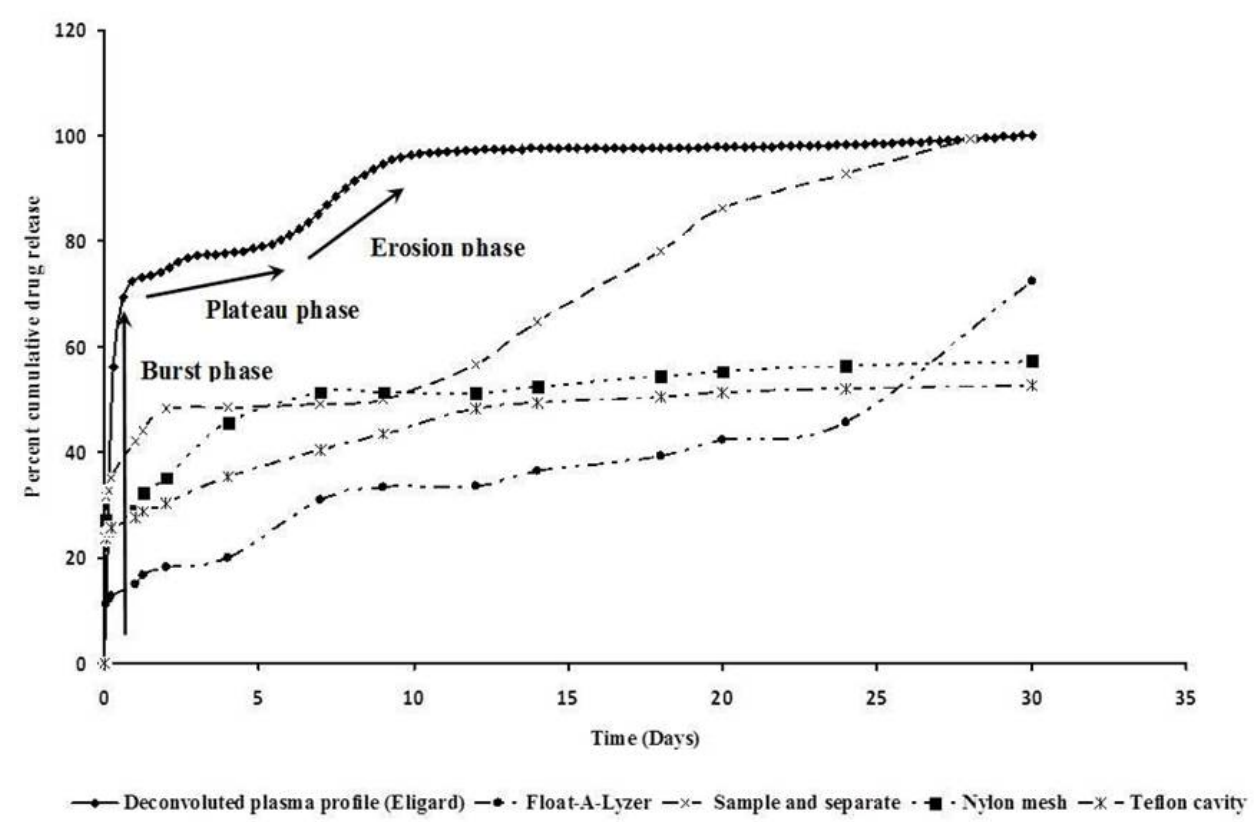

Figure 2-9. In vitro drug release using different experimental set ups and comparison with the deconvoluted plasma profile. 
Table 2-5. Comparison between 2-piece regression and 3-piece regression methods for correlating in vitro with in vivo drug release.

\begin{tabular}{lcc}
\hline In vitro method & \multicolumn{2}{c}{$\mathbf{R}^{\mathbf{2}}$ value } \\
\cline { 2 - 3 } & 2-piece regression & 3-piece regression \\
\hline & & \\
Sample and separate & 0.939 & 0.991 \\
Float-A-Lyzer & 0.8707 & 0.9834 \\
Nylon mesh & 0.9157 & 0.9148 \\
Teflon cavity & 0.9671 & 0.9711 \\
\hline
\end{tabular}


Table 2-6. Comparison of various in vitro test methods with respect to prediction error by 3-piece regression model.

\begin{tabular}{|c|c|c|c|c|}
\hline \multirow{2}{*}{$\begin{array}{l}\text { Time } \\
\text { (Days) }\end{array}$} & \multicolumn{4}{|c|}{ Prediction error } \\
\hline & $\begin{array}{c}\text { Sample and } \\
\text { separate } \\
\end{array}$ & Float-A-Lyzer & Nylon mesh & Teflon cavity \\
\hline 0 & -2.8349 & 0.39376 & $-2.00 \mathrm{E}-08$ & 0.52805 \\
\hline 0.3 & -1.7577 & -4.993 & -6.3487 & -9.1357 \\
\hline 0.6 & 7.28335 & 3.83596 & 5.50925 & 2.09898 \\
\hline 0.9 & 6.24336 & 2.58391 & 7.28623 & 3.25268 \\
\hline 1.2 & 4.34992 & 2.58947 & 7.19252 & 2.76077 \\
\hline 1.5 & 1.65156 & 0.9532 & 3.5552 & 0.49523 \\
\hline 1.8 & -0.3465 & 0.76663 & 2.82137 & 0.29971 \\
\hline 2.1 & -3.319 & 0.51128 & 1.74514 & 0.16055 \\
\hline 2.4 & -2.3735 & 1.15716 & 0.85593 & 0.30539 \\
\hline 2.7 & -1.6729 & 1.55804 & -0.2783 & 0.20523 \\
\hline 3 & -1.3144 & 1.61692 & -1.7545 & -0.2369 \\
\hline 3.3 & -1.2428 & 1.38881 & -3.5177 & -0.9661 \\
\hline 3.6 & -1.2183 & 1.11369 & -5.3279 & -1.7423 \\
\hline 3.9 & -1.1487 & 0.88357 & -7.0931 & -2.4734 \\
\hline 4.2 & -0.9853 & 0.84716 & -8.5354 & -2.4734 \\
\hline 4.5 & -0.7915 & -0.2953 & -8.5354 & 3.1171 \\
\hline 4.8 & -0.5228 & -1.3628 & -8.843 & -3.3153 \\
\hline 5.1 & -0.4688 & -2.3543 & -9.0745 & -3.4375 \\
\hline 5.4 & -0.7233 & -3.2278 & -9.1881 & -3.4417 \\
\hline 5.7 & -0.8019 & -0.39252 & -9.1256 & -3.2699 \\
\hline 6 & -0.6904 & -4.4327 & -8.8732 & -2.9081 \\
\hline 6.3 & -0.383 & -4.7442 & -8.4247 & -2.3504 \\
\hline 6.6 & 0.11943 & -4.8607 & -7.7813 & -1.5976 \\
\hline 6.9 & 0.81787 & -4.7811 & -6.9418 & -0.6488 \\
\hline 7.2 & 1.69231 & -4.5256 & -5.9264 & 0.476 \\
\hline 7.5 & 1.34933 & -3.2846 & -4.2519 & 1.62327 \\
\hline 7.8 & 0.87835 & -2.1716 & -2.7054 & 2.64253 \\
\hline 8.1 & 0.25037 & -1.2155 & -1.3159 & 3.5048 \\
\hline 8.4 & -0.5416 & -0.4235 & -0.0904 & 4.20306 \\
\hline 8.7 & -1.4986 & 0.20352 & 0.97011 & 4.73633 \\
\hline 9 & -1.8632 & 0.37224 & 1.86128 & 2.74877 \\
\hline 9.3 & -1.1611 & 1.05169 & 2.59878 & 4.91003 \\
\hline 9.6 & -0.6251 & 1.56514 & 3.17029 & 2.9053 \\
\hline 9.9 & -0.256 & 1.91159 & 3.5748 & 4.73356 \\
\hline 10.2 & -0.0439 & 2.10104 & 3.82231 & 4.40483 \\
\hline 10.5 & 0.08911 & 2.21114 & 3.99082 & 3.99709 \\
\hline
\end{tabular}


Table 2-6. Continued.

\begin{tabular}{|c|c|c|c|c|}
\hline \multirow{2}{*}{$\begin{array}{l}\text { Time } \\
\text { (Days) }\end{array}$} & \multicolumn{4}{|c|}{ Prediction error } \\
\hline & $\begin{array}{c}\text { Sample and } \\
\text { separate }\end{array}$ & $\begin{array}{c}\text { Float-A- } \\
\text { Lyzer }\end{array}$ & Nylon mesh & Teflon cavity \\
\hline 10.8 & 0.19418 & 2.29394 & 2.29394 & 3.56135 \\
\hline 11.1 & 0.28524 & 2.36239 & 4.25783 & 3.11162 \\
\hline 11.4 & 0.3633 & 2.41784 & 4.37134 & 2.64888 \\
\hline 11.7 & 0.43136 & 2.46329 & 4.47495 & 2.17615 \\
\hline 12.0 & 0.48742 & 2.49674 & 4.56636 & 1.69141 \\
\hline 12.3 & 0.50615 & 2.0503 & 4.43581 & 1.59646 \\
\hline 12.6 & 0.51288 & 1.59186 & 4.29326 & 1.4895 \\
\hline 12.9 & 0.50861 & 1.12242 & 4.13972 & 1.37155 \\
\hline 13.2 & 0.49434 & 0.64298 & 3.97617 & 1.24359 \\
\hline 13.5 & 0.46807 & 0.15154 & 3.80063 & 1.10364 \\
\hline 13.8 & 0.43079 & 0.07788 & 3.61408 & 0.95268 \\
\hline 14.1 & 0.40261 & 0.06951 & 3.48638 & 0.94971 \\
\hline 14.4 & 0.35905 & 0.05723 & 3.31292 & 0.76679 \\
\hline 14.7 & 0.31448 & 0.04395 & 3.13846 & 0.68386 \\
\hline 15.0 & 0.27092 & 0.03168 & 2.965 & 0.60193 \\
\hline 15.3 & 0.22835 & 0.0204 & 2.79254 & 0.52101 \\
\hline 15.6 & 0.18678 & 0.01012 & 2.62107 & 0.22108 \\
\hline 15.9 & 0.14722 & 0.00184 & 2.45161 & 0.36316 \\
\hline 16.2 & 0.10766 & -0.0064 & 2.28215 & 0.28523 \\
\hline 16.5 & 0.07109 & -0.0117 & 2.11569 & 0.2103 \\
\hline 16.8 & 0.03452 & -0.017 & 1.94923 & 0.13538 \\
\hline 17.1 & $-4.00 \mathrm{E}-05$ & -0.0203 & 1.78476 & 0.06245 \\
\hline 17.4 & -0.0346 & -0.0235 & 1.6203 & -0.0105 \\
\hline 17.7 & -0.0662 & -0.0238 & 1.45884 & -0.0804 \\
\hline 18.0 & -0.1133 & -0.0292 & 1.23856 & -0.1786 \\
\hline 18.3 & -0.1529 & -0.0425 & 1.07595 & -0.3344 \\
\hline 18.6 & -0.1916 & -0.0549 & 0.91435 & -0.4899 \\
\hline 18.9 & -0.2292 & -0.0662 & 0.75374 & -0.3428 \\
\hline 19.2 & -0.2659 & -0.0766 & 0.59414 & -0.7955 \\
\hline 19.5 & -0.3006 & -0.849 & 0.43653 & -0.9463 \\
\hline 19.8 & -0.3342 & -0.0923 & 0.278993 & -1.096 \\
\hline 20.1 & -0.348 & -0.0885 & 0.18419 & -1.1872 \\
\hline 20.4 & -0.3449 & -0.08 & 0.11895 & -1.2142 \\
\hline 20.7 & -0.3408 & -0.0706 & 0.05471 & -1.2403 \\
\hline 21.0 & -0.3357 & -0.0601 & -0.0085 & -1.2654 \\
\hline 21.3 & -0.3297 & -0.0487 & -0.0708 & -1.2895 \\
\hline 21.6 & -0.3216 & -0.0352 & -0.131 & -1.3116 \\
\hline
\end{tabular}


Table 2-6. Continued.

\begin{tabular}{|c|c|c|c|c|}
\hline \multirow{2}{*}{$\begin{array}{l}\text { Time } \\
\text { (Days) }\end{array}$} & \multicolumn{4}{|c|}{ Prediction error } \\
\hline & $\begin{array}{c}\text { Sample and } \\
\text { separate }\end{array}$ & $\begin{array}{c}\text { Float-A- } \\
\text { Lyzer }\end{array}$ & Nylon mesh & Teflon cavity \\
\hline 21.9 & -0.3125 & -0.0207 & -0.1902 & -1.3366 \\
\hline 21.9 & -0.3125 & -0.0207 & -0.1902 & -1.3366 \\
\hline 22.2 & -0.3024 & -0.0053 & -0.2485 & -1.3527 \\
\hline 22.5 & -0.2893 & 0.01319 & -0.3037 & -1.3698 \\
\hline 22.8 & -0.2723 & 0.03565 & -0.355 & -1.3829 \\
\hline 23.1 & -0.2532 & 0.06011 & -0.4042 & -1.3939 \\
\hline 23.4 & -0.2291 & 0.08957 & -0.4484 & -1.4 \\
\hline 23.7 & -0.202 & 0.12203 & -0.4897 & -1.4031 \\
\hline 24 & -0.1786 & 0.15265 & -0.5573 & -1.4199 \\
\hline 24.3 & -0.1442 & 0.11784 & -0.5551 & -1.4012 \\
\hline 24.6 & -0.1059 & 0.08703 & -0.5489 & -1.3786 \\
\hline 24.9 & -0.0365 & 0.06022 & -0.5387 & -1.3519 \\
\hline 25.2 & -0.0184 & 0.03641 & -0.5255 & -1.3223 \\
\hline 25.5 & 0.03118 & 0.0166 & -0.5082 & -1.2886 \\
\hline 25.8 & 0.08353 & -0.0002 & -0.488 & -1.252 \\
\hline 26.1 & 0.13987 & -0.013 & -0.4638 & -1.2113 \\
\hline 26.4 & 0.20022 & -0.0218 & -0.4356 & -1.1666 \\
\hline 26.7 & 0.26357 & -0.0276 & -0.4044 & -1.119 \\
\hline 27 & 0.33092 & -0.0295 & -0.3691 & -1.0673 \\
\hline 27.3 & 0.40227 & -0.0273 & -0.3299 & -1.0117 \\
\hline 27.6 & 0.47661 & -0.0221 & -0.22877 & -0.953 \\
\hline 27.9 & 0.55496 & -0.0129 & -0.2415 & -0.8904 \\
\hline 28.2 & 0.65241 & 0.00031 & -0.1912 & -0.8237 \\
\hline
\end{tabular}


using a set of preliminary PLGA formulations. The composition of such preliminary formulations was designed in order to include parameters such as drug loading, PLGA viscosity grade and vehicle composition in the study. The comparison between various in vitro test methods at three different drug loadings i.e. $4 \%, 8 \%$ and $16 \% \mathrm{w} / \mathrm{w}$ of polymer is depicted in Figure 2-10. The overall response of PLGA micro particulate formulations to various in vitro test methods at different drug loadings was essentially same i.e. highest cumulative percent drug release by the sample and separate method and a delayed final erosion phase by techniques such as dialysis, nylon mesh and Teflon cavity based in vitro drug release tests. The Float-A-Lyzer seemed to have restricted the drug diffusion into the dissolution medium in some way. This essentially means that the dialysis membrane was somehow controlling or limiting the drug release further. This can be explained by the following series of events. Upon inserting the Float-A-Lyzer tube containing the formulation inside the dissolution medium, the dissolution medium starts to diffuse into the Float-A-Lyzer tube. Drug begins to dissolve in the dissolution medium inside the tube and as the concentration starts to build up, drug starts diffusing out into the external dissolution medium. However, dialysis being an equilibrium phenomenon, only allows the diffusion of drug up to a certain extent after which the drug release plateaus off due to insufficient drug inside the dialysis tube containing formulation. This necessitates the constant maintenance of sink conditions in the outer compartment and constant sampling but could result in drug concentrations much below the quantitation limit. Figure 2-11 shows the separation of formulation components inside the Float-ALyzer after some time, which was further responsible for incomplete drug release and an early plateau level when using a dialysis membrane apparatus. As the vehicle/carrier separated out from the micro particles, there was insufficient solvent available for the plasticization of micro particles. Micro particles began to settle at the bottom of the dialysis tube due to high density and formed an incomplete plasticized particle aggregate that almost looked like a small pellet (Figure 2-12). Such a hard pellet like structure was further responsible for slowing down the drug release. Due to a slower rate of PLGA polymer gelling in the Teflon cavity, the micro particles settled down thereby forming a layer of gelled particles at the bottom of the cavity. This resulted in an insufficient interaction between the polymer particles and the plasticizer in the vehicle, thereby altering the drug release. Figure 2-13 shows the individual components of the Teflon cavity set up and the layer of gelled PLGA micro particles.

Comparison of two different PLGA viscosity grades further resulted in similar conclusions (Figure 2-14). Changing the vehicle composition from ATEC (acetyl triethyl citrate) + NMP (N-methyl pyrrolidone) + GMO (glyceryl monooleate) to ATEC alone resulted in similar drug release profiles obtained from sample and separate method, nylon mesh method and Teflon cavity method (Figure 2-15). The slowest drug release was obtained by using dialysis method. This could be explained by the fact that a high amount of ATEC resulted in the formation of a fluid polymer matrix over a short period of time leading to a much faster drug release. Such faster drug release resulted in the development of relatively higher local concentrations of drug in the vicinity of the formulation from where it could readily diffuse into the outer and the bigger dissolution media compartment. This phenomenon explains the similar drug release obtained by using sample and separate, nylon mesh and Teflon cavity methods. In the dialysis method 


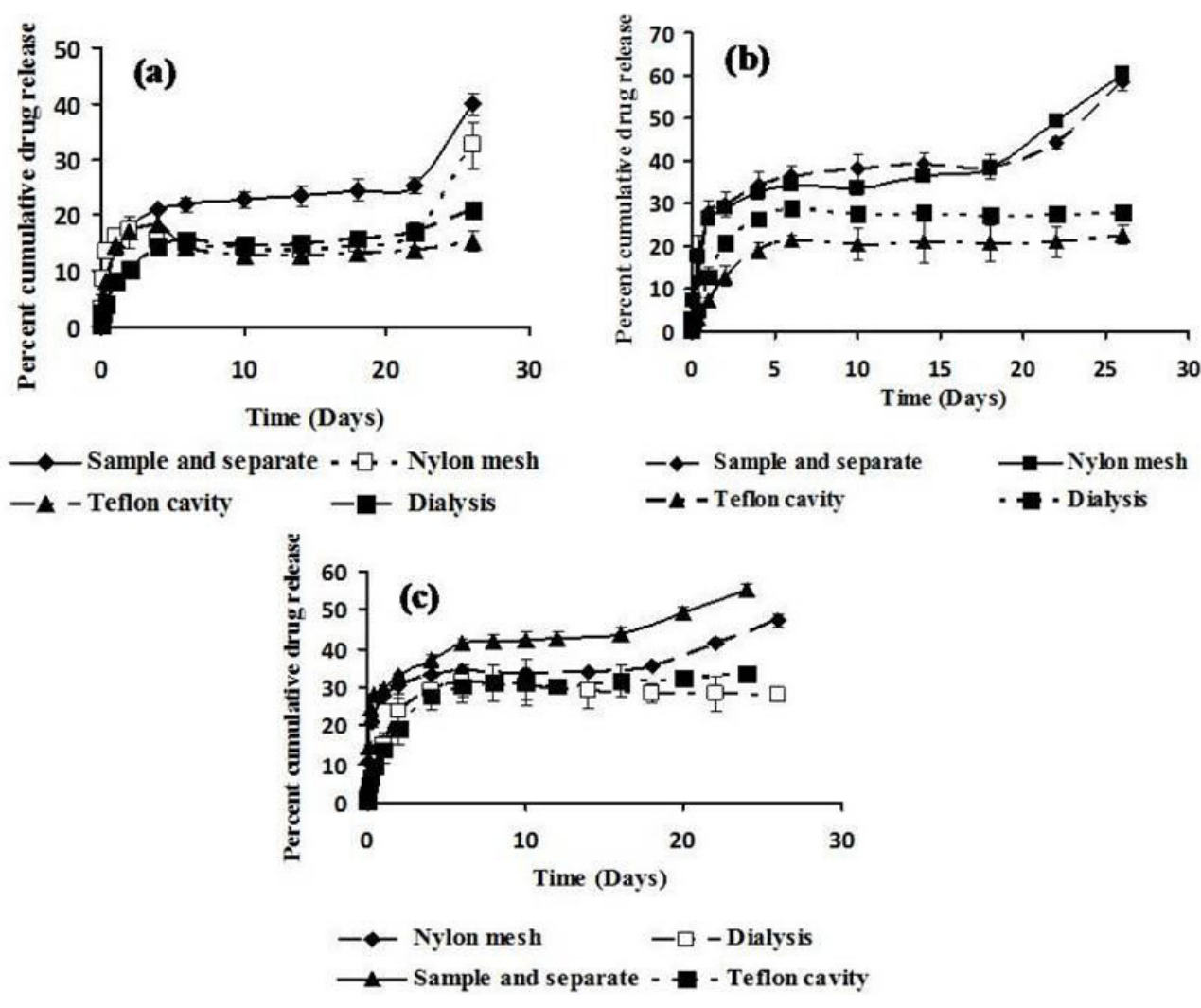

Figure 2-10. Comparison of drug release from PLGA based micro particulate formulation using four different in vitro test methods at the drug loadings of (a) $4 \% \mathrm{w} / \mathrm{w}$ of polymer; (b) $8 \% \mathrm{w} / \mathrm{w}$ of polymer; and (c) $16 \% \mathrm{w} / \mathrm{w}$ of polymer. 


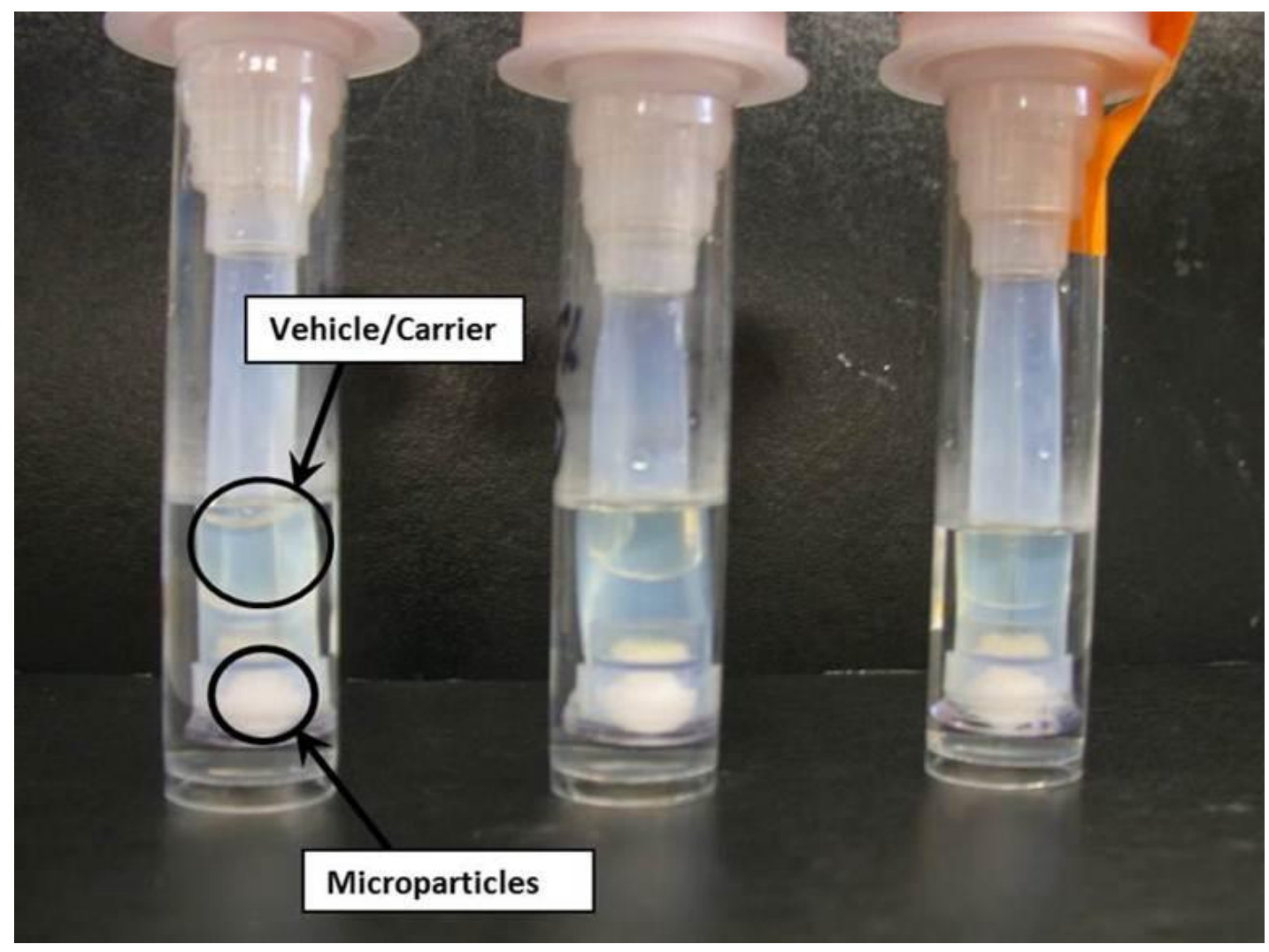

Figure 2-11. Separation of formulation components in the Float-A-Lyzer. 


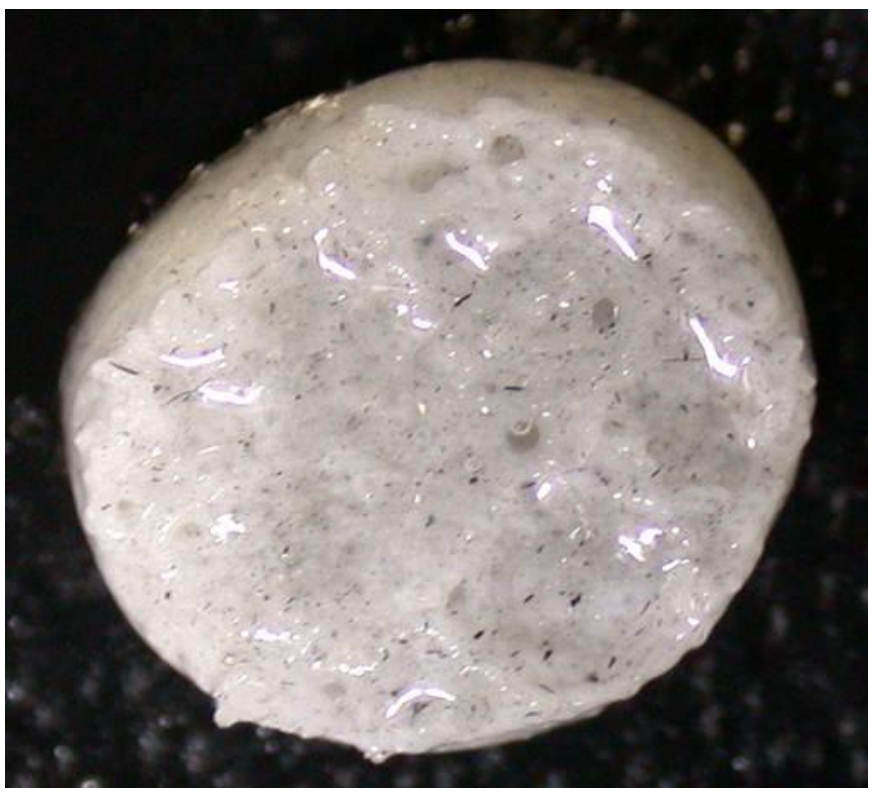

Figure 2-12. Micro particle aggregates formed at the bottom of the Float-A-Lyzer. 

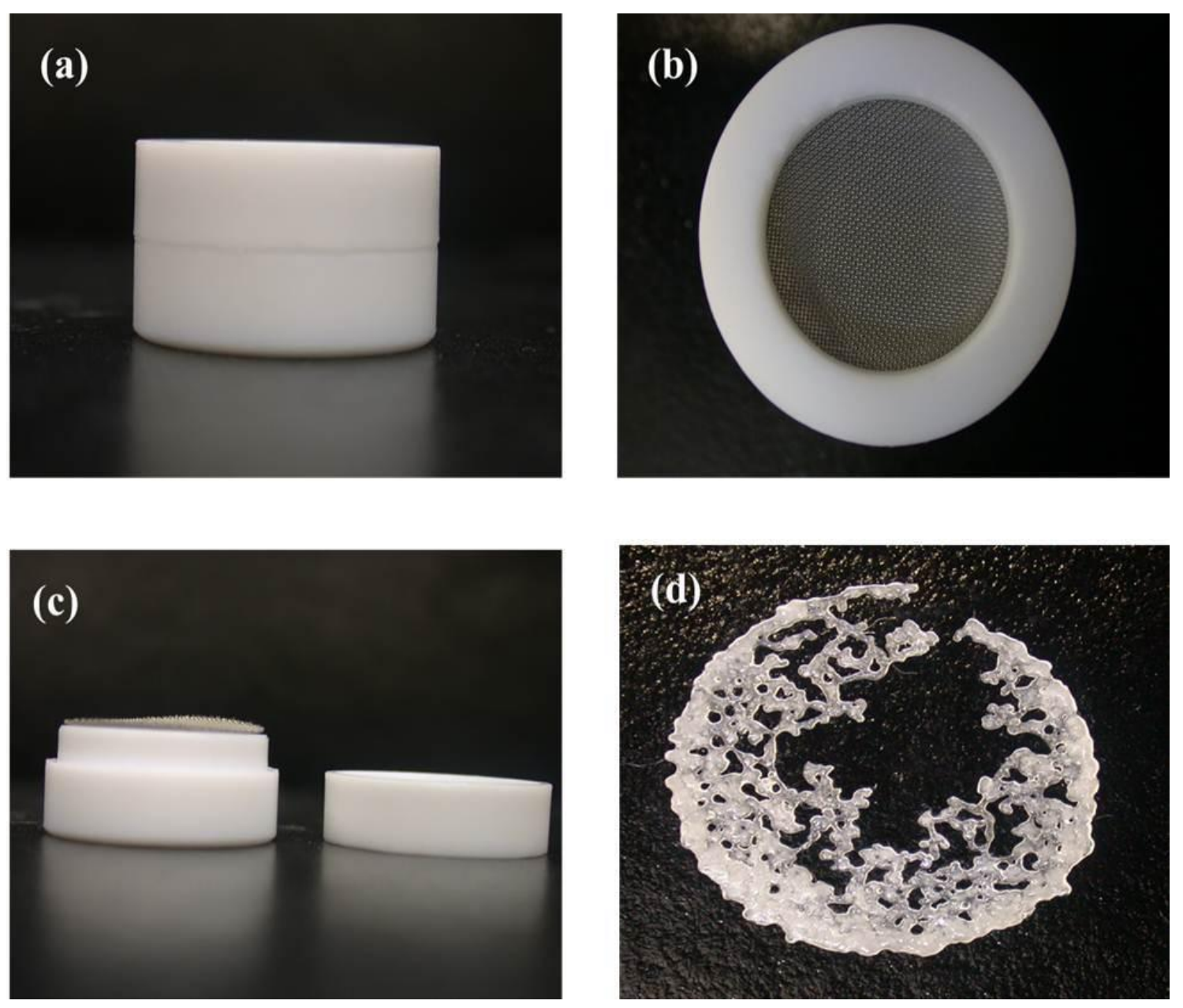

Figure 2-13. Design of the Teflon Cavity used for drug release study (a) side view of the Teflon Cavity; (b) top view of the Teflon cavity; (c) split view of the Teflon cavity; and (d) micro particle aggregates at the bottom of Teflon cavity. 

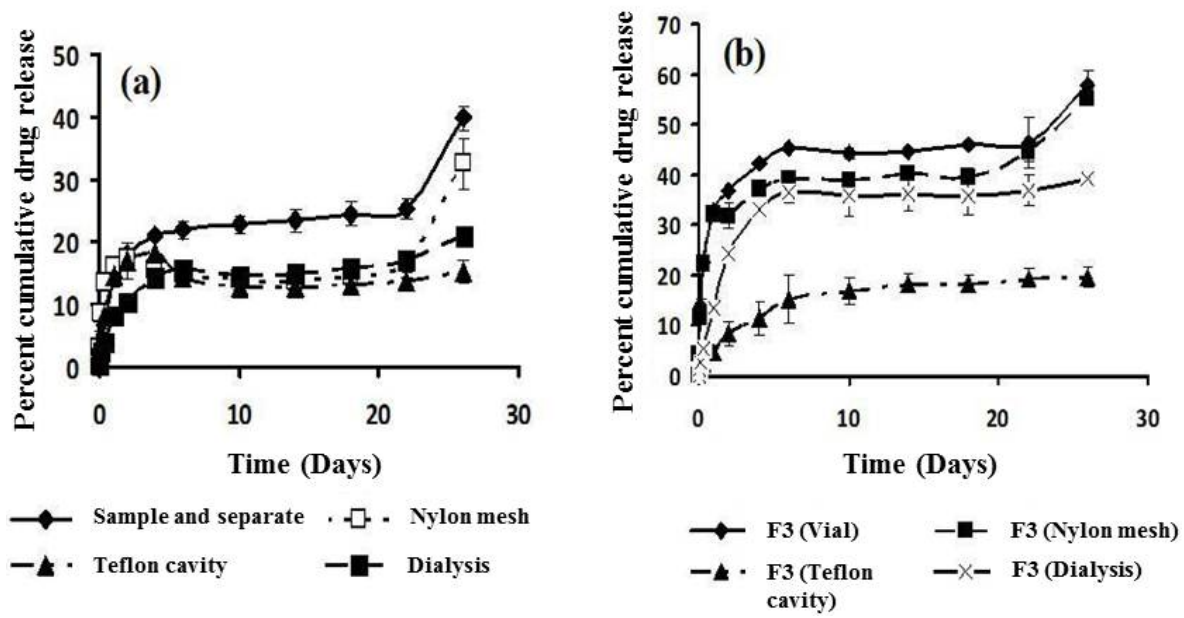

Figure 2-14. Comparison of drug release from PLGA based micro particulate formulation using four different in vitro test methods at PLGA viscosity grades of (a) $0.24 \mathrm{dL} / \mathrm{g}$; and (b) $0.59 \mathrm{dL} / \mathrm{g}$. 


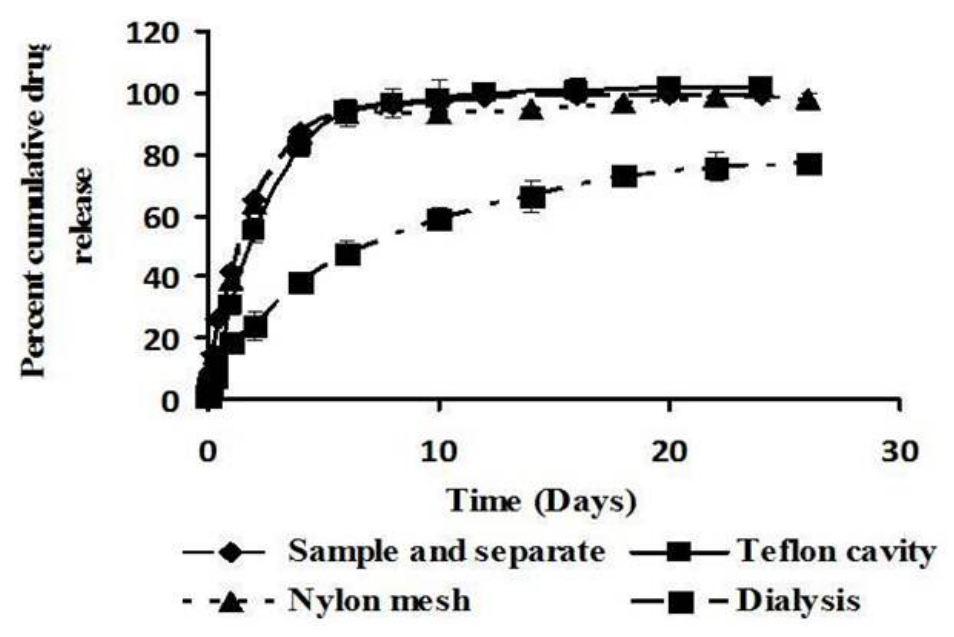

Figure 2-15. Comparison of drug release from PLGA based micro particulate formulation containing a single plasticizer (ATEC) using four different in vitro test methods. 
set up, however, the drug still took time to diffuse out of the dialysis tube despite of initial high drug concentrations inside the tube. This could be due to a relatively smaller pore size of the dialysis membrane compared to the nylon mesh and the stainless steel mesh of Teflon cavity and therefore, a lower diffusion rate of the drug into the outer compartment.

\subsection{Conclusion}

An in vitro release test method based on the sample and separate set up was most appropriate and predictive of the in vivo dissolution for the marketed PLGA based formulation i.e. Eligard $\AA$. The drug release from other experimental set ups was hindered mostly by an obstruction in the drug diffusion mechanism resulting in a slower drug release from such systems. All three phases of drug release i.e. burst phase, plateau phase and erosion phase were well captured by the sample and separate method of in vitro drug release. Further, a simple 3-piece regression model could describe the correlation between in vitro and in vivo drug release very well. The validity of the sample and separate method was also confirmed by testing drug release from some of the preliminary in house PLGA based formulations, whereby a highest and unobstructed drug release was obtained again by the sample and separate method. We therefore, selected the aforementioned method for future in vitro optimization and mechanistic studies of the formulations. 


\section{CHAPTER 3. PREFORMULATION AND PRELIMINARY SCREENING STUDIES FOR FORMULATION DEVELOPMENT}

\subsection{Introduction}

Early formulation work, which involves conducting preformulation studies as well as preliminary screening studies for various excipients in the formulation, is extremely critical from the standpoint of drug development process. Formulators try to screen the excipients from various perspectives such as drug solubility and stability etc. Early preformulation work provides an idea of the future success of the final formulation. Not paying attention to the preformulation studies, on the other hand, could result in major formulation failures such as drug instability or significant discrepancies in the drug release characteristics. Nonetheless, the process starts with characterizing the drug substance as well as various excipients to be included in the formulation.

Various preformulation studies have been employed by formulators all over the world that are designed specifically for the drug product under development. These include drug solubility determination, characterization of the polymorphic form of the drug, understanding drug-polymer interactions if any etc. Furthermore, formulation ingredients are selected based on the desired release characteristics of the formulation. For instance, major focus would be on the excipients that can retard the drug release if the final objective is to have a controlled release formulation. Various techniques can be used for the purpose of preformulation screening studies. These include differential scanning calorimetry (DSC), fourier-transform infrared spectroscopy (FT-IR), X-ray powder diffraction (XRPD) etc.

The in situ PLGA polymeric implants (IPPIs) are formulated as drug loaded PLGA micro particles, which upon dispersing into the vehicle or injection results in formation of a polymeric implant at the site of injection. Micro particles have been used as prolonged release systems for several drugs, including antimicrobial, chemotherapic, and anti-inflammatory agents. ${ }^{102-104}$ Poly (DL-lactide-co-glycolide) (PLGA) is a copolymer of lactic and glycolic acid widely used in particular drug release systems owing to its biodegradability and biocompatibility. ${ }^{45,105-107}$ Factors affecting drug release e.g. solid state solubility of the drug in polymer have been studied well by researchers as a part of the preformulation screening studies. ${ }^{108}$ Further since, the drug has to be encapsulated inside the polymeric particles, it becomes extremely important for us to understand the potential stability issues of such drug-loaded polymer micro particles. The preliminary study on compatibility between drug and the polymer would provide indications of any potential stability issue. The effect of the spray drying process on the drug-polymer interactions between triamcinolone and PLGA and the stability of micro particles has been studied by using differential scanning calorimetry (DSC), thermo gravimetry (TG) and derivative thermo gravimetry (DTG), X-ray powder diffraction (XRPD), and fourier-transform infrared spectroscopy (FT-IR). ${ }^{109}$ Moreover, it is also important to test the stability of drug in the vehicles/carrier used to disperse these micro particles. Since the present system has been formulated essentially to keep the drug 
separate from the vehicle for injection taking the potential stability issues into considerations, it is not necessary to test the drug stability in various solvents for the vehicle under accelerated conditions. Drug stability is a concern in some solvents such as $\mathrm{N}$-methyl pyrrolidone (NMP) and therefore the drug product manufacturers claim $100 \%$

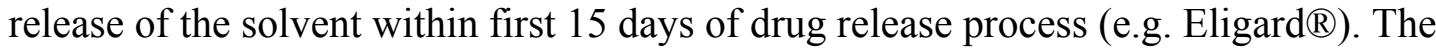
drug, however, still remains in contact with the vehicle solvent during the drug release process for some period of time. It, therefore calls for determining the drug stability in various vehicle solvents at the conditions used for determining the drug release behavior i.e. $37^{\circ} \mathrm{C}$ in the dissolution media.

The present study focuses on characterization of drug and drug product by various techniques such as DSC, FT-IR, XRPD, drug solubility and stability studies in various vehicle solvents and preliminary screening studies for the vehicle solvents.

\subsection{Materials and Methods}

\subsubsection{Materials}

N-methyl pyrrolidone (Pharmasolve, NMP) was procured from was procured from ISP Pharma Technologies, Wayne, NJ, USA. Triethyl citrate (TEC), acetyl triethyl citrate (ATEC), and acetyl tributyl citrate (ATBC) were obtained from Morflex Inc., Greensboro, NC, USA and polyethylene glycol 400 (PEG 400) was received from The Dow Chemical Co., Midland, MI, USA. Poly lactide-co-glycolide (PLGA, $0.59 \mathrm{dL} / \mathrm{g}$ ) purchased from Lactel absorbable polymers, Durect Corporation, Pelham, AL, USA was the polymer selected for making the drug loaded micro particles. Leuprolide acetate (nonapeptide) procured from Teva Pharmaceuticals, Israel was selected as the model drug. The structure of drug and various vehicle solvents is presented in Figure 3-1. All the solvents and buffer reagents were of analytical grade.

\subsubsection{Methods}

\subsubsection{Analytical method development}

An appropriate amount of leuprolide was dissolved in reverse osmosis (RO) water to obtain a stock solution of $1000 \mu \mathrm{g} / \mathrm{ml}$ concentration. Appropriate aliquots were withdrawn and diluted to $10 \mathrm{ml}$ each in order to obtain leuprolide standards at various concentration levels. The standards were obtained in the concentration range of $5 \mu \mathrm{g} / \mathrm{ml}$ to $50 \mu \mathrm{g} / \mathrm{ml}$. Leuprolide Acetate was analyzed by reverse phase (RP)-HPLC using a C18 column, $150 \mathrm{~mm} \times 4.60 \mathrm{~mm}$ (Phenomenex, Torrance, CA). A gradient elution method was used with mobile phase A $(0.1 \%$ [ $\mathrm{vol} / \mathrm{vol}]$ trifluoroacetic acid in water $)$ and mobile phase B $(0.1 \%$ [vol/vol] trifluoroacetic acid in acetonitrile). The gradient was set as $10 \%$ to $20 \%$ solvent B during first $2.5 \mathrm{~min}, 20 \%$ to $27 \%$ solvent B from 2.5 to $6 \mathrm{~min}, 27 \%$ to 

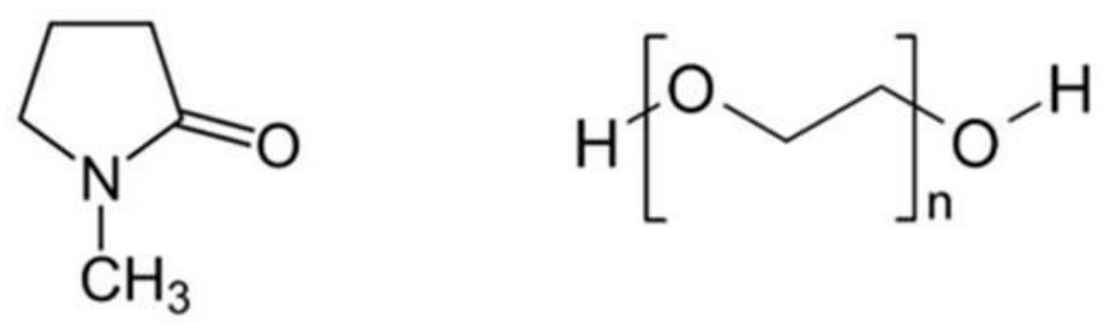

$\mathrm{N}$-methyl-2-pyrrolidone $\left(\mathrm{C}_{5} \mathrm{H}_{9} \mathrm{NO}\right)$

Polyethylene glycol $\left(\mathrm{C}_{2 n+2} \mathrm{H}_{4 n+6} \mathrm{O}_{n+2}\right)$

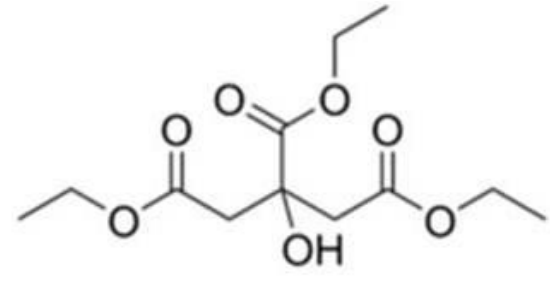

Triethyl citrate $\left(\mathrm{C}_{12} \mathrm{H}_{20} \mathrm{O}_{7}\right)$

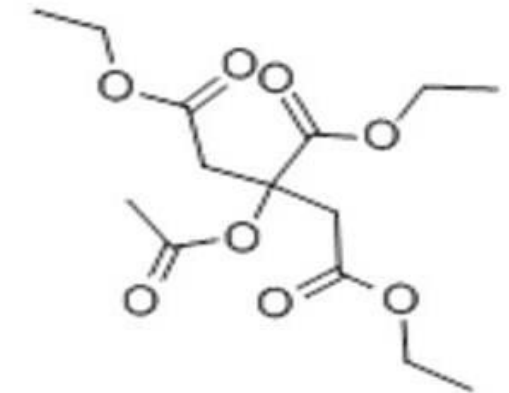

Acetyl triethyl citrate $\left(\mathrm{C}_{14} \mathrm{H}_{22} \mathrm{O}_{8}\right)$

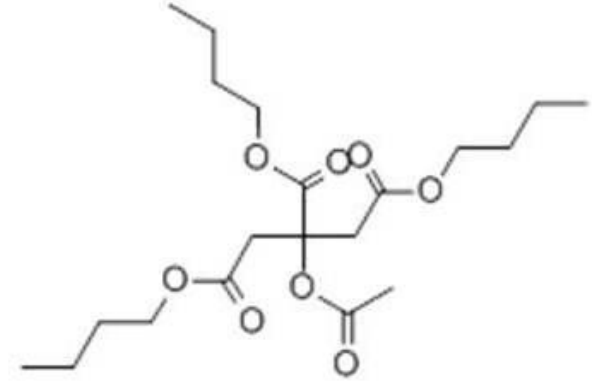

Acetyl tributyl citrate $\left(\mathrm{C}_{20} \mathrm{H}_{34} \mathrm{O}_{8}\right)$

Figure 3-1. Structures and chemical formula of various vehicle solvents. 
$60 \%$ solvent B from 6 to $8 \mathrm{~min}$, a constant flow of $60 \%$ solvent $\mathrm{B}$ from 8 to $15 \mathrm{~min}$ and $60 \%$ to $10 \%$ solvent B from 15 to $20 \mathrm{~min}$. The flow rate was set at $1.5 \mathrm{~mL} / \mathrm{min}$ and UV absorbance was measured at $215 \mathrm{~nm}$ using a photodiode array detector equipped with the high performance liquid chromatography system (HPLC). Standard curve was plotted at the aforementioned concentration range resulting in a good linearity value $\left(\mathrm{R}^{2}=0.9998\right)$.

\subsubsection{Micro particle preparation and preformulation studies}

Drug loaded micro particles were prepared by the film grinding method. Briefly, the polymer was dissolved in acetone in a ratio of 1:10 for the polymer to acetone. This was followed by dispersing the drug into polymer solution on a magnetic stirrer. Once the drug was uniformly dispersed in the polymer solution, the solvent i.e. acetone was allowed to evaporate resulting in a semi-solid flow able mass. This residual mass was then transferred to a vacuum oven set at $50^{\circ} \mathrm{C}$ and $25 \mathrm{in} \mathrm{Hg}$ of pressure after drying at room temperature for first 24 hours. After vacuum drying the drug-polymer mass for another 24 hours, the resulting polymer film was then ground to micro particles by using a cryo-mill.

Briefly, the milling procedure involved first placing the film in the cryo mill jar which was then immersed in the liquid nitrogen for 10 minutes. Once the liquid nitrogen stops bubbling, the jar is taken out and fixed in place in the mill. The mill is then operated for about 4 minutes at the frequency of $28 \mathrm{~Hz}$. The drug-polymer micro particles thus obtained were subjected to various characterization tests such as DSC, FT-IR, and XRPD. DSC studies were conducted in the MDSC mode using a scanning rate of $3^{\circ} \mathrm{C} / \mathrm{min}$, modulation temperature amplitude of $\pm 1.00^{\circ} \mathrm{C}$ and sinusoidal modulation of 60 sec. FT-IR studies were done on a PerkinElmer Spectrum ${ }^{\text {TM }} 100$ FT-IR spectrometer using the Analyst software. XRPD studies were done using a Bruker D8 powder XRPD instrument.

\subsubsection{Drug solubility studies}

Equilibrium solubility of drug was determined in various vehicle solvents such as NMP, TEC, ATEC, ATBC and PEG 400. A known excess of drug (20 mg) was added to $2 \mathrm{ml}$ of each of the above solvents and allowed to stir for 72 hours in a shaker bath maintained at $37^{\circ} \mathrm{C}$. The study was conducted in triplicates and samples were withdrawn at 24, 48 and 72 hours respectively for drug content determination by HPLC. For the hydrophilic solvents such as NMP and PEG 400, the sample withdrawn was directly dissolved in water for drug quantification purposes. The hydrophobic solvents, on the other hand, were allowed to equilibrate with water and the drug was extracted out in the water layer and quantified by HPLC. The water used for drug extraction was pre-equilibrated with the corresponding solvent before being used for extraction process. 


\subsubsection{Drug stability studies}

\subsection{Drug stability in phosphate buffer saline (PBS), pH 7.4}

A known amount of drug was dissolved in phosphate buffer prepared according to Table 2-3 (Chapter 2) in order to result in a final drug concentration of $50 \mu \mathrm{g} / \mathrm{ml}$. The drug solution was then divided into two separate parts. First part was left as it is and $0.2 \%$ of sodium azide was added to the second part. The study was done in triplicates and the individual solutions were transferred to tightly capped glass bottles. The samples were then loaded into a shaker bath maintained at a temperature of $37^{\circ} \mathrm{C} .1 \mathrm{ml}$ samples were withdrawn periodically and drug content was determined by HPLC.

\subsection{Drug stability in vehicle solvents}

The PBS, pH 7.4 was equilibrated with all the hydrophobic vehicle solvents individually by agitating excess of the solvent with buffer. The hydrophilic solvents were added to the buffer resulting in a concentration of $100 \mu \mathrm{g} / \mathrm{ml}$ each. A known amount of drug was then added to above solutions to result in a concentration of $50 \mu \mathrm{g} / \mathrm{ml}$. The samples were transferred to shaker bath maintained at $37^{\circ} \mathrm{C}$. Samples were withdrawn periodically and injected into HPLC to determine the drug content.

\subsection{Drug stability in polymer micro particles}

Drug loaded polymer micro particles were subjected to analysis by FT-IR and sodium dodecyl sulphate polyacrylamide gel electrophoresis (SDS-PAGE) in order to establish structural stability of the drug in these micro particles. Drug-polymer micro particles were simply compressed into a small pellet on the diamond crystal window in the FT-IR equipment and spectra were recorded for drug as well as drug loaded micro particles.

SDS-PAGE analysis was conducted by using ready gel Tris-tricine peptide gels. Such gels are optimized for separating peptides and proteins with low molecular weights. Drug was extracted from polymer micro particles by using methylene chloride-water liquid-liquid extraction system (Figure 3-2). $50 \mu 1$ of the drug solution was diluted with an equal volume of sample buffer (Table 3-1) followed by heating at $95^{\circ} \mathrm{C}$ for 5 minutes. $20 \mu 1$ of the aforementioned diluted drug solution was then transferred to the individual wells in the tris-tricine gel. $20 \mu \mathrm{l}$ of a reference drug solution was also added to one of the adjacent wells after similar treatment. The gel was run at constant $100 \mathrm{~V}$ for 60 minutes by using the running buffer specified in Table 3-2. Drug stability was further confirmed by subjecting the drug extracted from both the ester end group and acid end group polymer micro particles to liquid chromatography mass spectrometry (LC-MS) analysis. The mass spectra for both the drug solutions were collected in order to determine the total molecular weight of the drug in two samples. 


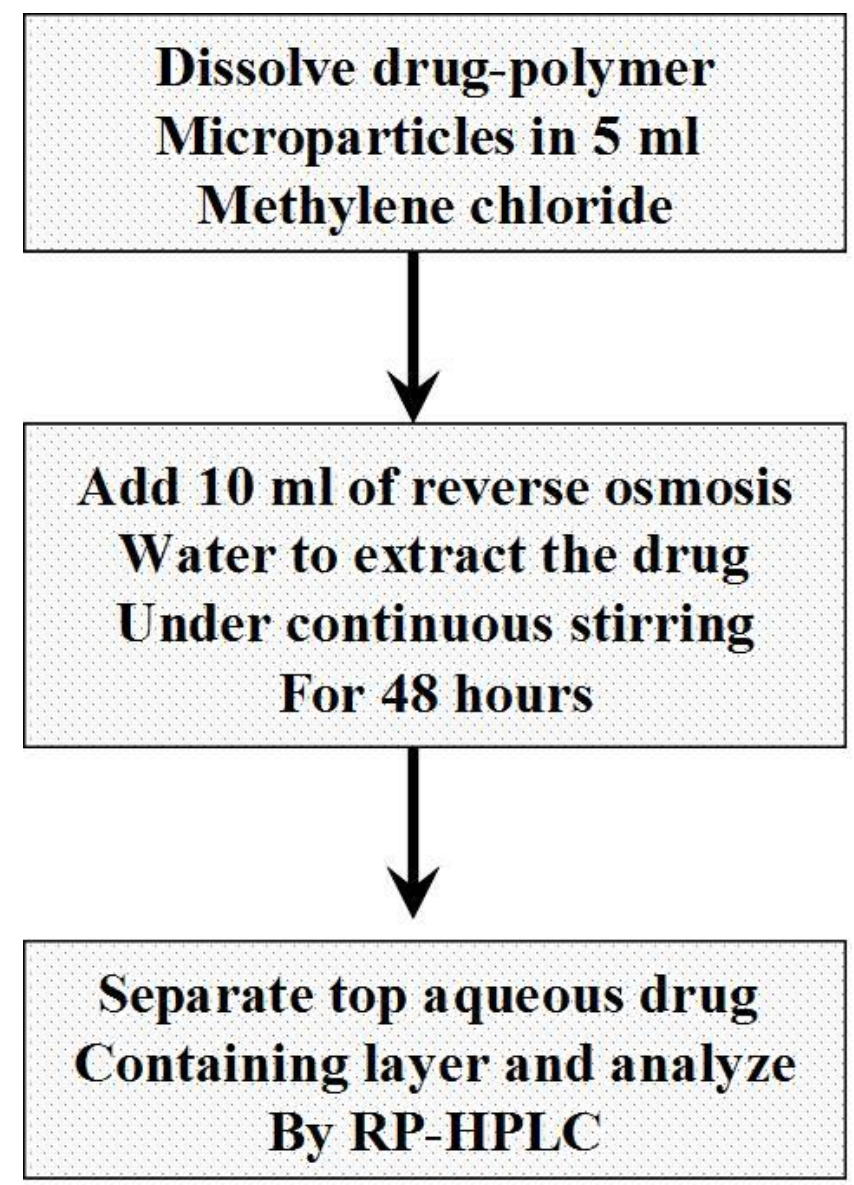

Figure 3-2. Liquid-liquid extraction method for determining drug loading in leuprolide containing PLGA micro particles. 
Table 3-1. Sample buffer for SDS-PAGE.

\begin{tabular}{lc}
\hline Working concentration & 2x stock \\
\hline & \\
$200 \mathrm{mM}$ Tris $\mathrm{HCl}, \mathrm{pH} 6.8$ & $1.0 \mathrm{mM}$ Tris HCl, $\mathrm{pH} 6.8(2.0 \mathrm{ml})$ \\
$2 \% \mathrm{SDS}$ & $10 \% \mathrm{SDS}(2.0 \mathrm{ml})$ \\
$40 \%$ Glycerol & Glycerol $(4.0 \mathrm{ml})$ \\
$0.04 \%$ Coomassie blue G-250 & $0.5 \%$ Coomassie blue $\mathrm{G}-250(0.8 \mathrm{ml})$ \\
$2 \%$ 2-mercaptoethanol & 2-mercaptoethanol $(0.2 \mathrm{ml})$ \\
\hline
\end{tabular}


Table 3-2. Running buffer for SDS-PAGE.

\begin{tabular}{lc}
\hline Working concentration & 2x stock \\
\hline & \\
$100 \mathrm{mM}$ Tris & Tris base $(60.55 \mathrm{~g})$ \\
$100 \mathrm{mM}$ Tricine & Tricine $(89.60 \mathrm{~g})$ \\
$0.1 \% \mathrm{SDS}$ & SDS $(5.0 \mathrm{~g})$ \\
& To $500 \mathrm{ml}$ with deionized water \\
\hline
\end{tabular}




\subsubsection{Screening studies for vehicle solvent}

\subsection{Drug release study in PBS, $p H 7.4$}

TEC, ATEC and ATBC were chosen as hydrophobic plasticizers with ATBC $>$ ATEC > TEC as order of hydrophobicity and PEG (polyethylene glycol) was selected as hydrophilic plasticizer. PEG was combined with all three hydrophobic plasticizers at 100:0, 70:30, 30:70 and 0:100 (\% w/w). Drug loaded polymer micro particles were dispersed into corresponding injection vehicle solvents by using the two syringe system depicted in Figure 3-3.

Briefly, the drug-loaded micro particles corresponding to $7.5 \mathrm{mg}$ of drug were loaded onto one of the syringes with male luer lock. $800 \mu 1$ of the injection vehicle solvent was added to the second syringe with female luer lock. The contents of both the syringes were mixed uniformly by going back and forth in the syringes 3-5 times. The entire contents were then withdrawn into the syringe with male luer lock. A 19 gauge needle was attached to the tip of the syringe and the contents were emptied into $10 \mathrm{ml}$ of the dissolution media (PBS, $\mathrm{pH}$ 7.4). The formulation begins to form a gel after coming in contact with the media and sets in place. The vials are transferred to the shaker bath maintained at $37^{\circ} \mathrm{C}$ and $100 \mathrm{rpm} .1 \mathrm{ml}$ samples were withdrawn periodically with replacement of fresh buffer and drug release was determined by an in house developed HPLC method as discussed in Chapter 2. A C18 column and a combination of water and acetonitrile were used for the study.

\subsection{DSC studies for determination of glass transition temperature (Tg)}

Samples were prepared for DSC analysis similar to that for drug release studies. A small amount of the formulation was withdrawn from the vials with the help of a curved spatula and loaded onto the DSC pan corresponding to a weight of 10-20 mg. The remaining formulation was discarded and another vial of same formulation was sampled for next time point. The DSC study was conducted in the modulated differential scanning mode (MDSC) at the heating rate of $3^{\circ} \mathrm{C} / \mathrm{min}$, modulation temperature amplitude of \pm $1.00^{\circ} \mathrm{C}$ and sinusoidal modulation of $60 \mathrm{sec}$ in the temperature range of $-90^{\circ} \mathrm{C}$ to $60^{\circ} \mathrm{C}$.

\subsection{Results and Discussions}

\subsubsection{Analytical method development}

Figure 2-4 (Chapter 2) shows the standard curve for leuprolide with linearity $\left(\mathrm{R}^{2}\right)$ of 0.9998 in the concentration range of $5 \mu \mathrm{g} / \mathrm{ml}$ to $50 \mu \mathrm{g} / \mathrm{ml}$. Method validation results including intraday and interday variation and accuracy are given in Table 2-4 (Chapter 2). 


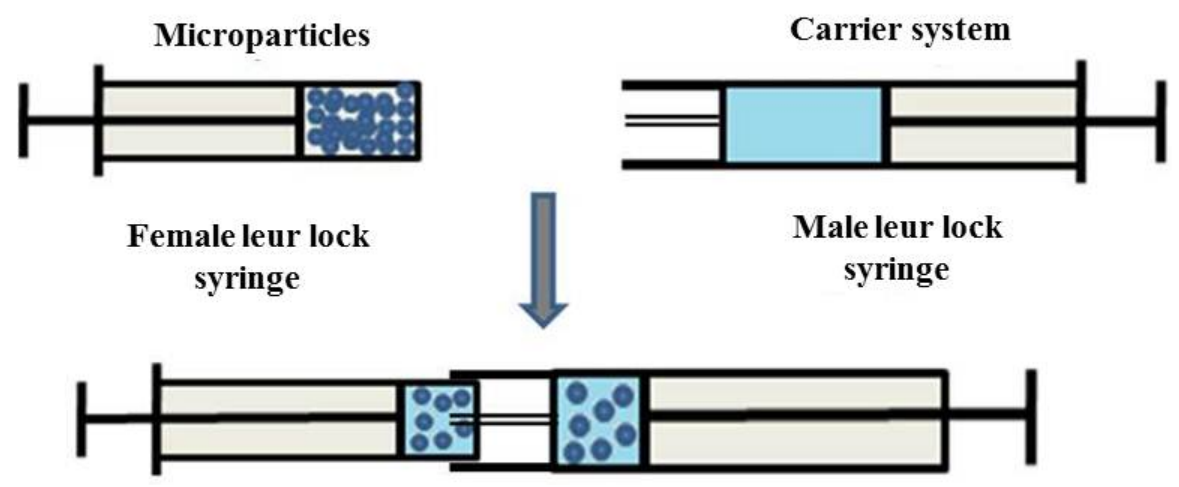

Figure 3-3. Two syringe system for in situ PLGA polymeric implant system (IPPIs). 


\subsubsection{Preformulation studies}

Leuprolide shows a drug degradation peak at around $213.39^{\circ} \mathrm{C}$ (Figure 3-4). The drug appears to be crystalline in nature to the naked eyes. However, the results obtained from X-ray diffraction analysis shows that the drug has an amorphous structure (Figure 3-5). Based on the results obtained from the XRPD analysis, it is clear that the endothermic peak in the DSC thermogram of leuprolide corresponds to the peptide degradation and not the melting peak of drug. The PLGA polymers with ester end group showed a distinct transition from a glassy state to a rubbery structure at the temperature corresponding to the $\mathrm{Tg}$ of the polymer i.e. $41.24^{\circ} \mathrm{C}$. This $\mathrm{Tg}$ was reduced to $25.26^{\circ} \mathrm{C}$ in the drug-loaded PLGA micro particles. This could be explained by the plasticizing effect of the drug on PLGA polymer that results in a significant reduction in Tg of polymer. A similar effect has been observed by Siepmann et al. where they quantify the plasticizing effect of ibuprofen, chlorpheniramine maleate and metoprolol tartrate on polyacrylate based films. ${ }^{10}$ The polymer, however, still retains an amorphous structure as depicted by the XRPD profile (Figure 3-6). Furthermore, the drug degradation peak was delayed and appeared at a relatively higher temperature $\left(286.61^{\circ} \mathrm{C}\right)$ in the drug polymer micro particles (Figure 3-7), which could be due to the protection provided by the polymer matrix to the drug against degradation. Drug- polymer interaction was ascertained from FT-IR studies. Figure 3-8 shows the FT-IR spectrum of drug, drug-polymer physical mixture and drug-polymer micro particles. The peak at $1746 \mathrm{~cm}^{-1}$ in the FT-IR spectrum of the polymer corresponds to the carbonyl stretching frequency of the ester bond and peak at $1626 \mathrm{~cm}^{-1}$ in the FT-IR spectrum of the drug corresponds to the carbonyl stretching frequency of the amide bond in the polypeptide backbone (amide I band). Further, the peak at $1535 \mathrm{~cm}^{-1}$ corresponds to the amide II region of the polypeptide representing contributions from $\mathrm{C}-\mathrm{N}$ stretching and $\mathrm{N}-\mathrm{H}$ bending modes. Amide II band especially provides important information regarding the hydrogen bonding between the peptide drug and polymer matrix. According to the results, there is no significant shift in the frequency of the amide II band in the drug-polymer micro particles made from PLGA polymer with ester end group. The polymer with the acid end group, however, resulted in a spectral shift from $1535 \mathrm{~cm}^{-1}$ to $1542 \mathrm{~cm}^{-1}$ indicating hydrogen interaction of $\mathrm{N}$ in amide II region with carboxylic group of PLGA. A similar interaction has been studied before between insulin and PLGA polymer by Hamishehkar et al. ${ }^{111}$ The results were further confirmed by visual examination of the semi-solid drug-polymer mass obtained after loading the drug into polymer solution (Figure 3-9). The drug loaded polymer matrix with the ester end group polymer appeared cloudy due to immiscibility of the drug in polymer matrix. Whereas, the drug polymer matrix prepared with the acid end group polymer exhibited a clear and transparent structure indicating miscibility of drug within the polymer matrix.

\subsubsection{Drug solubility studies}

Figure 3-10 shows the solubility results for leuprolide in various vehicle solvents. Based on the relative polarity values of various solvents, equilibrium solubility values for leuprolide were placed at different locations in the TEAS plot. Leuprolide shows an 


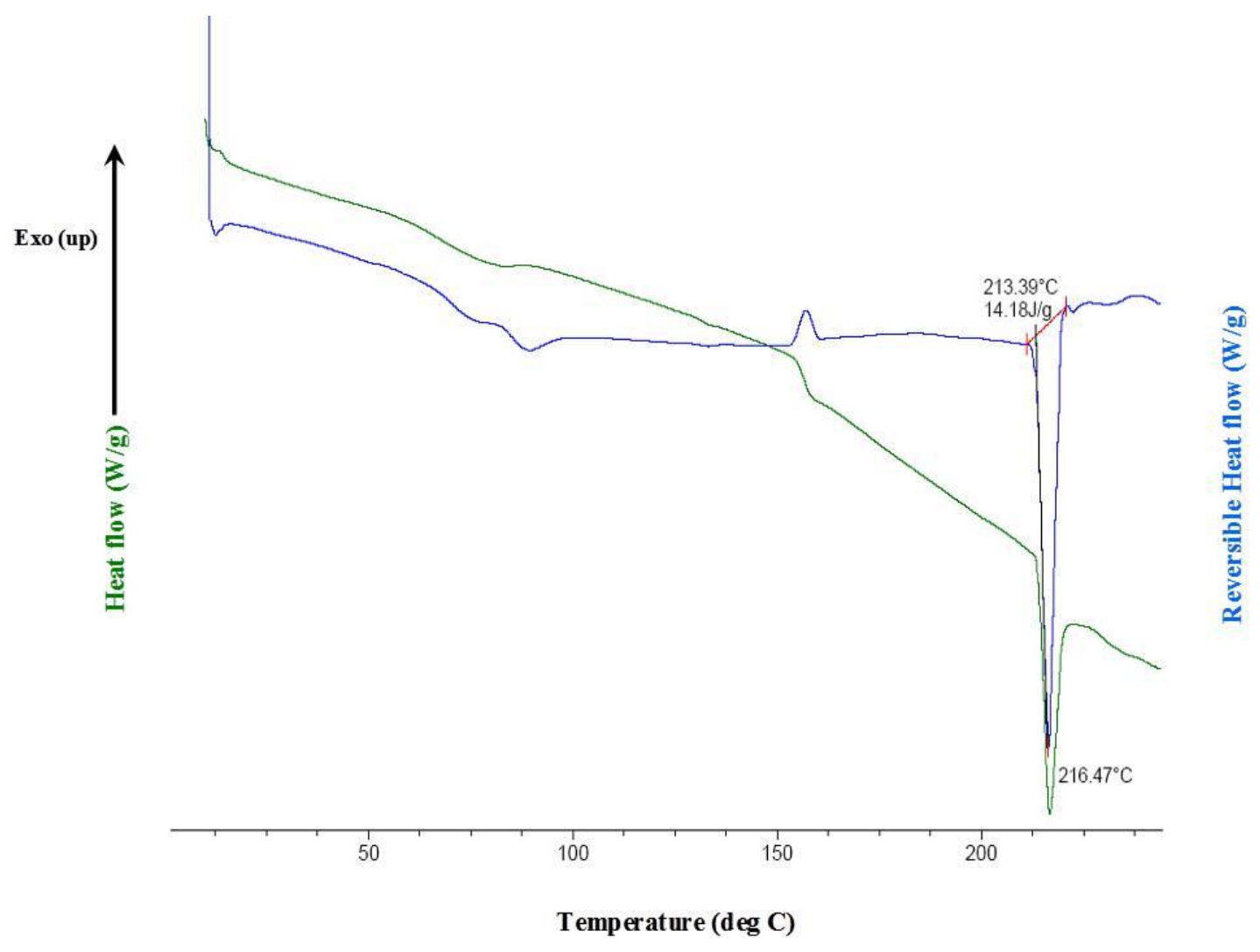

Figure 3-4. DSC thermogram of leuprolide. 


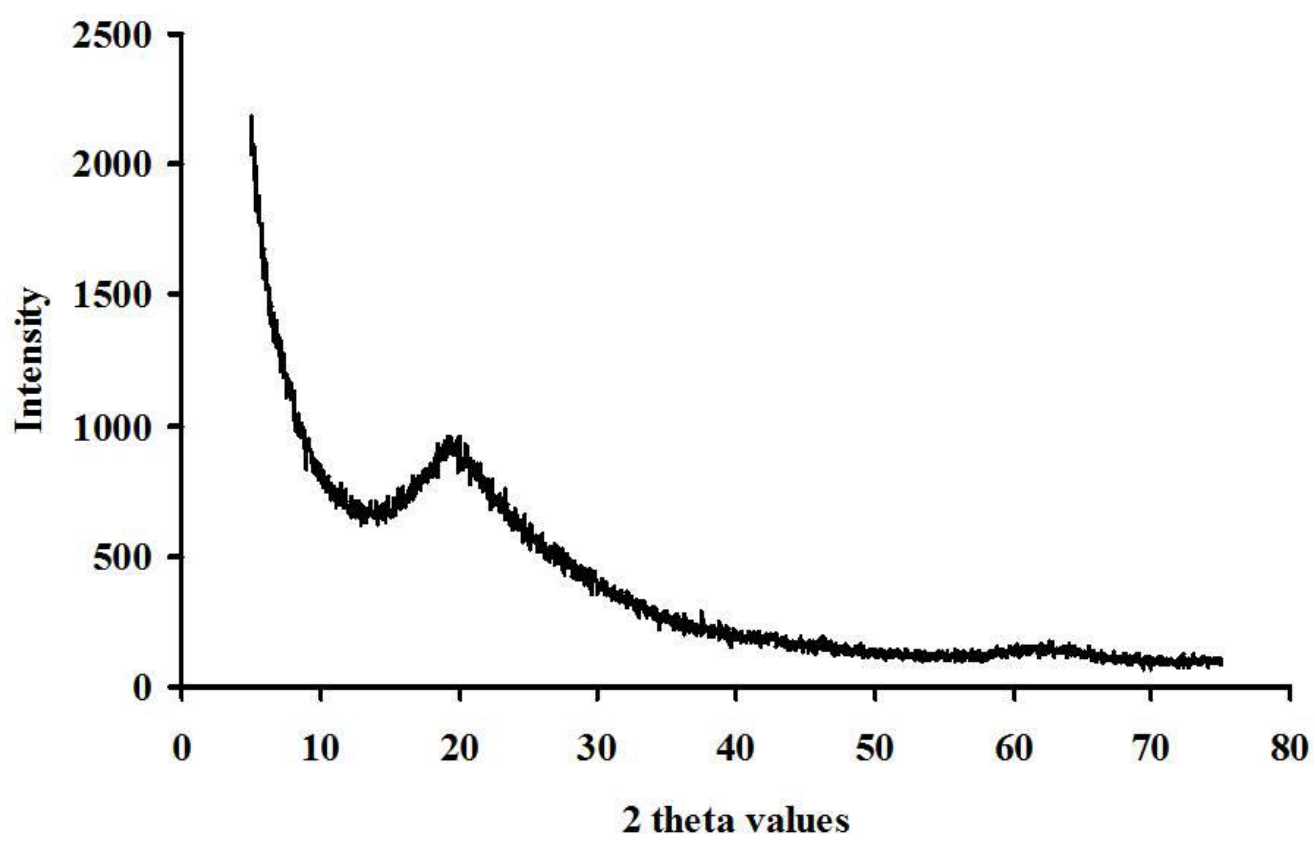

Figure 3-5. XRPD plot of leuprolide. 


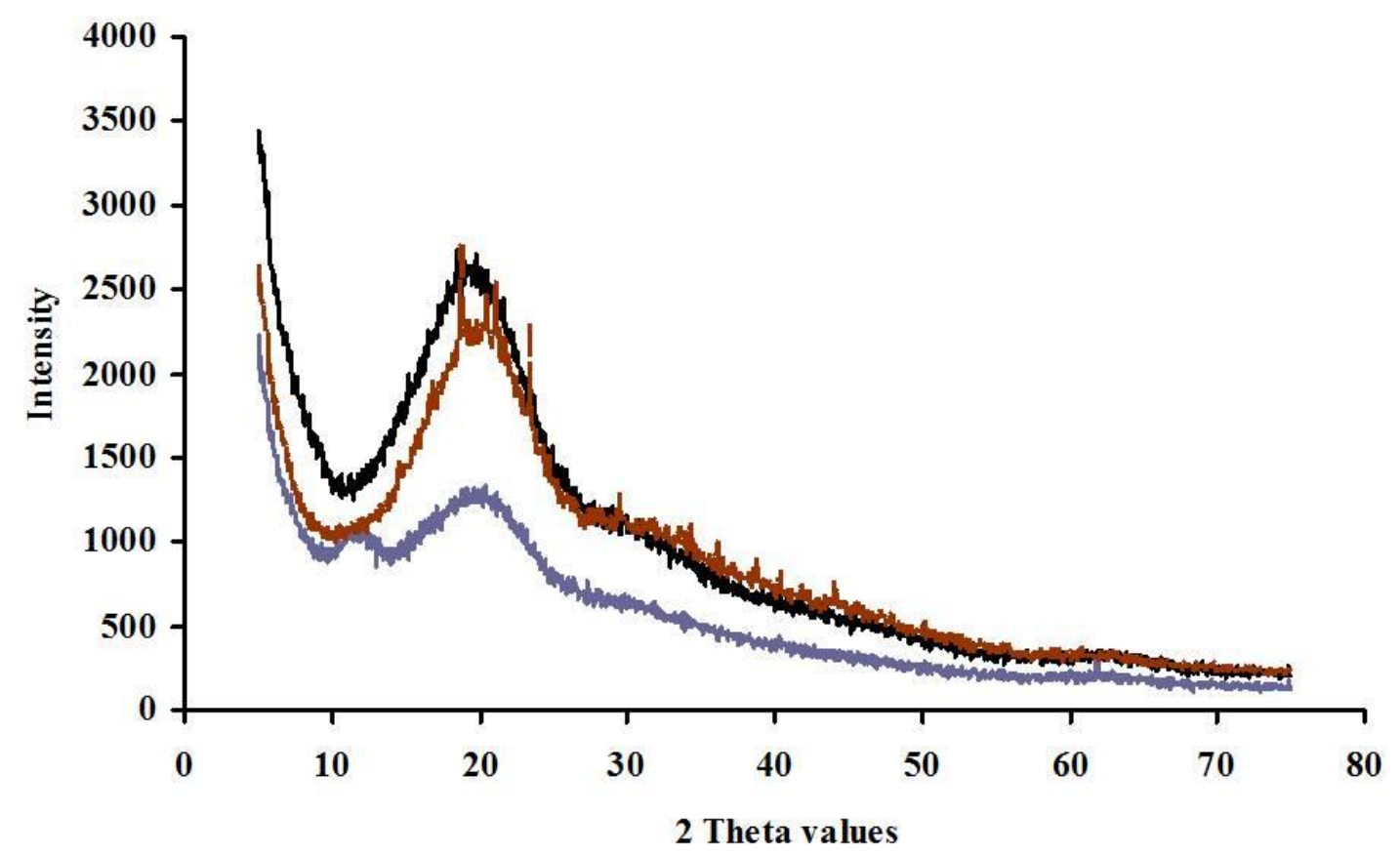

Leuprolide-PLGA-Ester particles L Leuprolide-PLGA-Acid particles

- - Blank PLGA-Es ter particles

Figure 3-6. XRPD plots of drug-loaded polymer micro particles. 

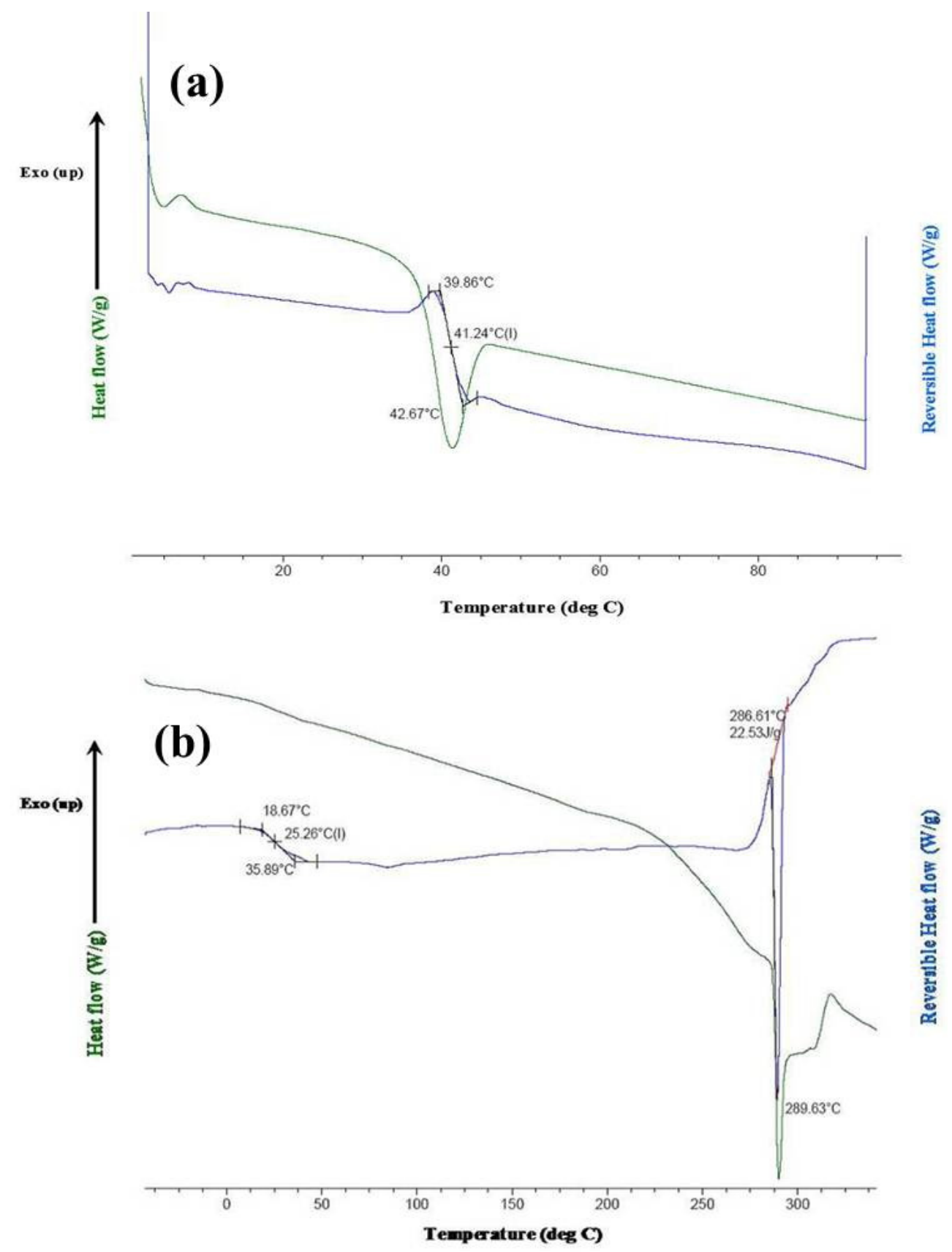

Figure 3-7. DSC thermograms of (a) PLGA polymer; and (b) drug-loaded polymer micro particles. 


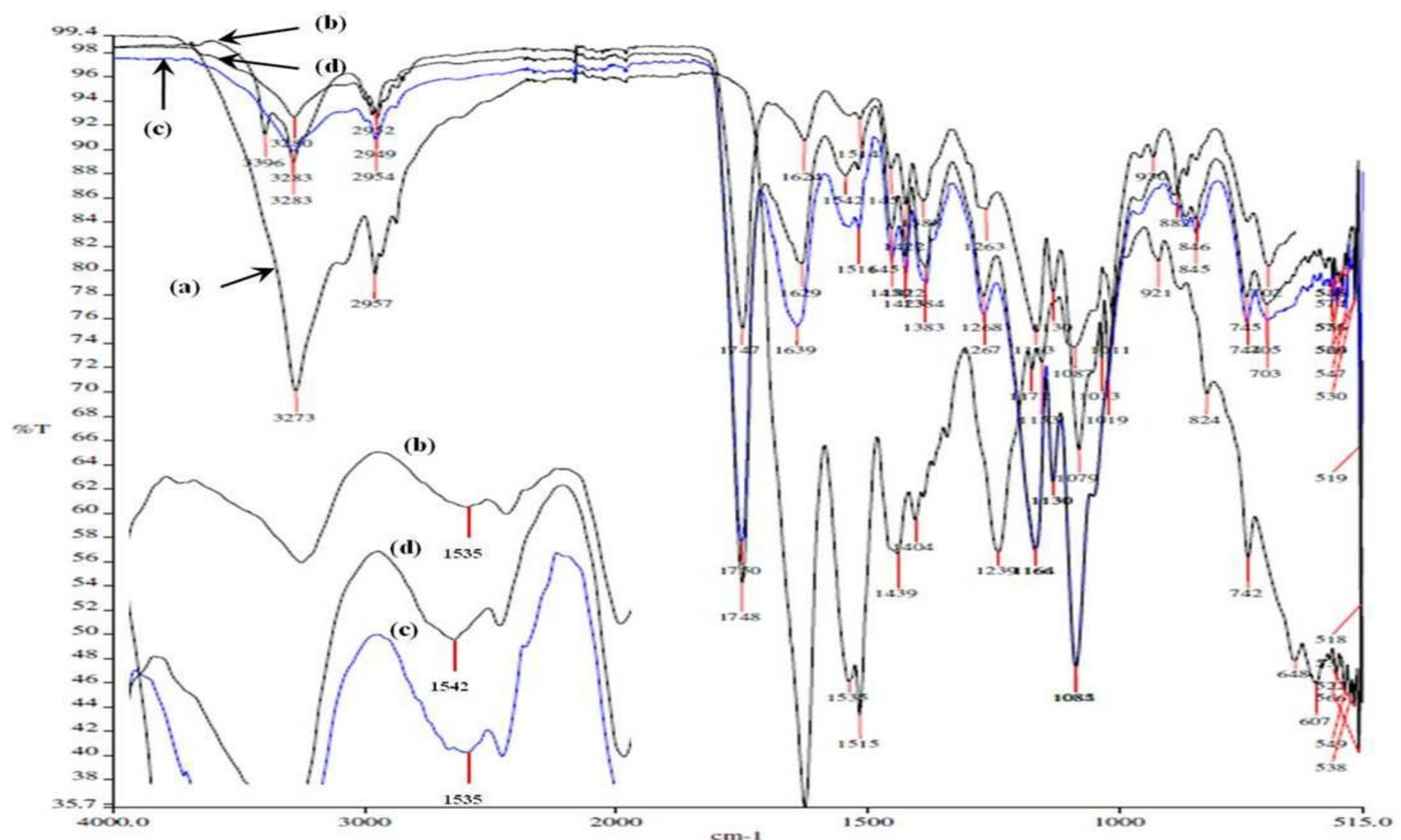

Figure 3-8. FT-IR spectra of (a) leuprolide; (b) leuprolide-PLGA-ester physical mixture; (c) drug-loaded PLGA-ester micro particles; and (d) drug-loaded PLGA-acid micro particles. 

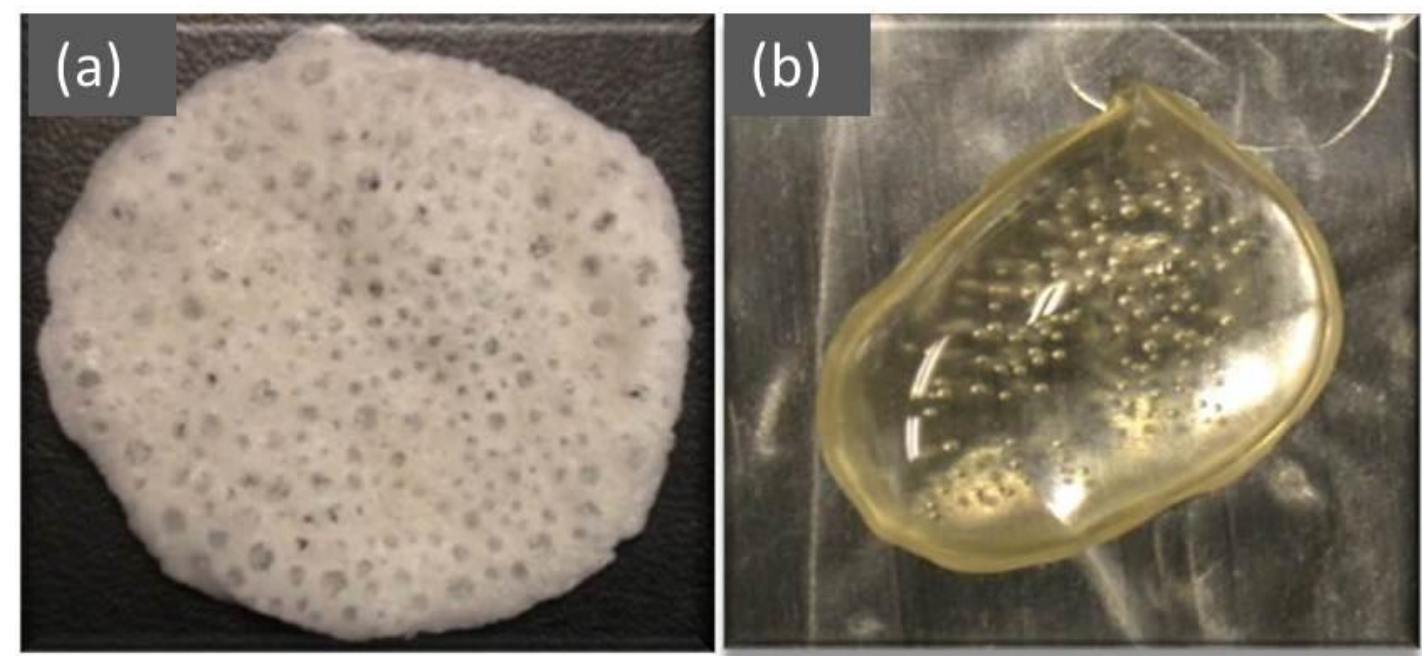

Figure 3-9. Visual comparison of drug-loaded polymer mass between (a) polymer with ester end group; and (b) polymer with acid end group. 


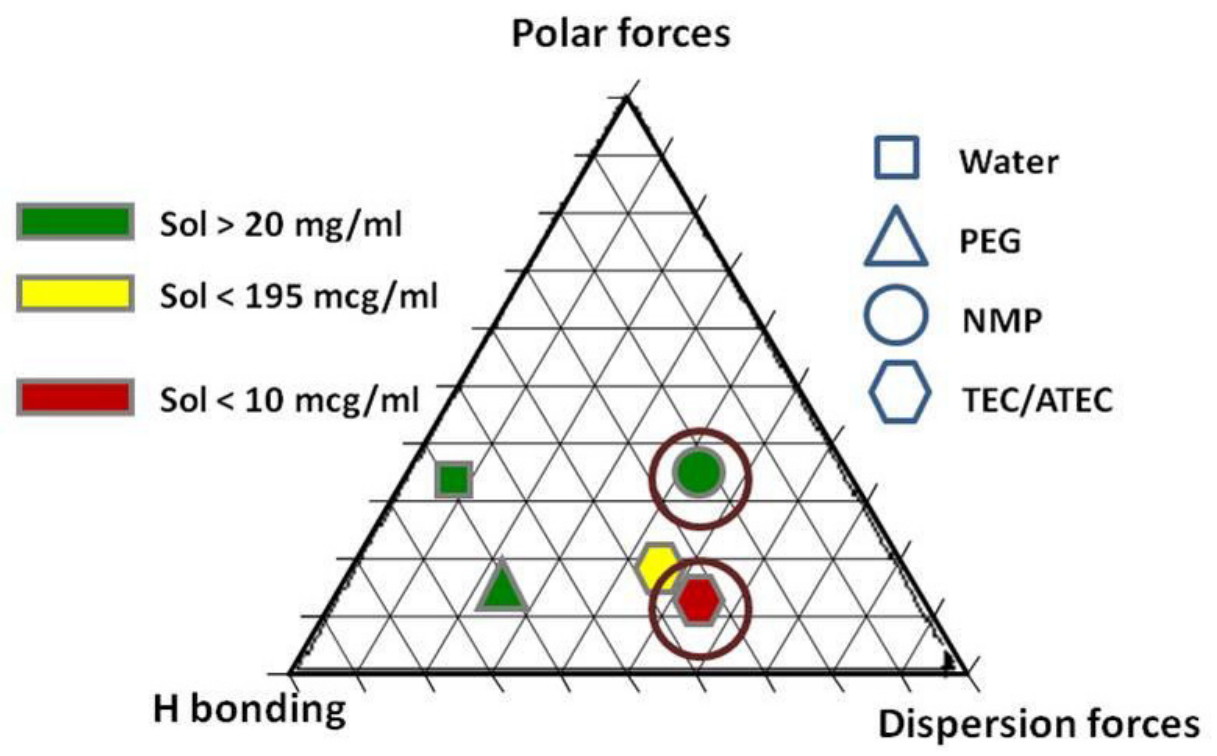

Figure 3-10. TEAS diagram for leuprolide solubility in various solvents/plasticizers. 
equilibrium solubility value of greater than $20 \mathrm{mg} / \mathrm{ml}$ in the hydrophilic solvents i.e. PEG and NMP. The solubility in TEC was less than $195 \mu \mathrm{g} / \mathrm{ml}$ and even less than $10 \mu \mathrm{g} / \mathrm{ml}$ in ATEC and ATBC.

\subsubsection{Drug stability studies}

The results for leuprolide stability in PBS, pH 7.4 as determined by HPLC shows that the drug is not stable and is probably oxidized fairly easily in aqueous conditions present in the PBS (Figure 3-11). The aqueous stability of the drug, however, is significantly improved in presence of sodium azide, which serves as an anti-microbial agent thus preventing further oxidation of the drug. Sufficient stability of the drug $(>99$ $\%$ ) could be achieved for more than 30 days in the aqueous environment in presence of $0.2 \%$ sodium azide.

Further, drug content did not seem to have changed significantly in the PBS upon addition of various vehicle solvents (Figure 3-12). This shows that although there would be a leaching out of the solvent from the formulation during the drug release process, it would not negatively impact the drug stability in the media.

The results for drug stability as determined in the polymer micro particles are depicted in Figure 3-8, Figure 3-13 and Figure 3-14. It can be clearly seen from the Figure 3-8 that although there is a slight shift in the amide II band wave number for the acid end group polymer micro particles, there is no other significant change in the peak characteristics for the drug encapsulated in micro particles. This slight shift in the amide II band as mentioned earlier is attributed to the hydrogen bonding between drug and polymer with acid end group and not necessarily due to structural changes in the drug molecule. SDS-PAGE further confirms the results (Figure 3-13), where the identical nature and position of the bands for the pure drug and drug in the micro particles can be observed clearly. The results were still confirmed by the LC-MS analysis of the drug extracted out from the micro particles made with both polymer containing ester and acid end groups respectively. LC-MS chromatograms show the mass/charge peak at around 1210, which represents the true molecular weight of the compound (Figure 3-14). This essentially shows that drug retains both its primary as well as secondary structure when formulated into micro particles with the PLGA polymer.

\subsubsection{Screening studies for vehicle solvents}

The idea behind choosing some of the solvents as the vehicle to suspend the micro particles for drug administration was to achieve polymer plasticization. In other words, once the solvent begins to interact with the polymer in the micro particles, the polymer chains start to relax. The degree of polymer chain relaxation depends on the overall affinity between polymer chains and the solvent with higher interaction resulting in more relaxation of polymer chains. Once the polymer chains are relaxed or extended, the drug can diffuse through these polymer chains into the outer aqueous medium. Based on the 


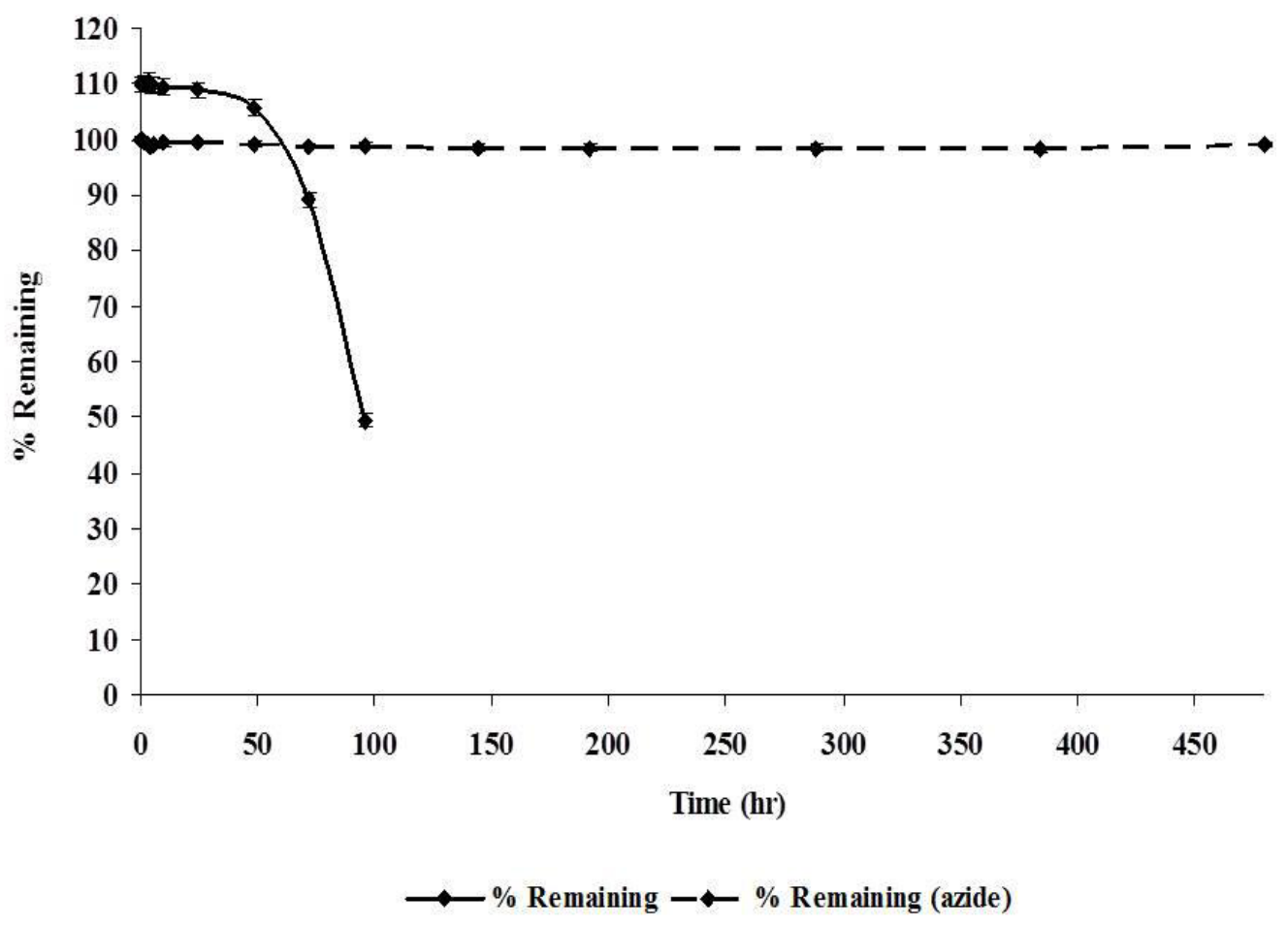

Figure 3-11. Leuprolide stability in PBS in absence and presence of $0.2 \%$ sodium azide.

Notes: Each data point represents an average of three measurements. Standard deviation of three measurements is presented as error bars. 


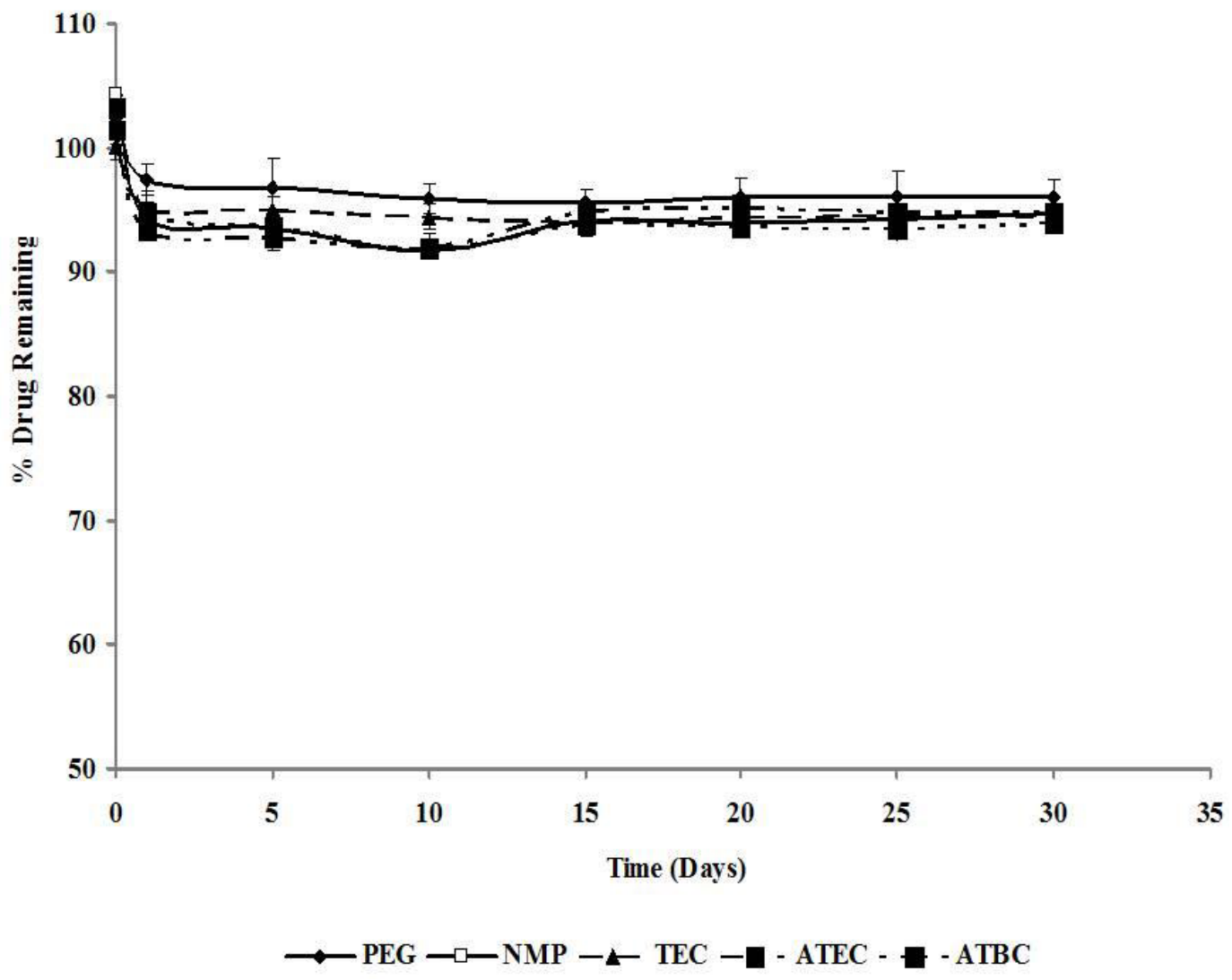

Figure 3-12. Drug stability in PBS in presence of various vehicle solvents/plasticizers.

Notes: Each data point represents an average of three measurements. Standard deviation of three measurements is presented as error bars. 


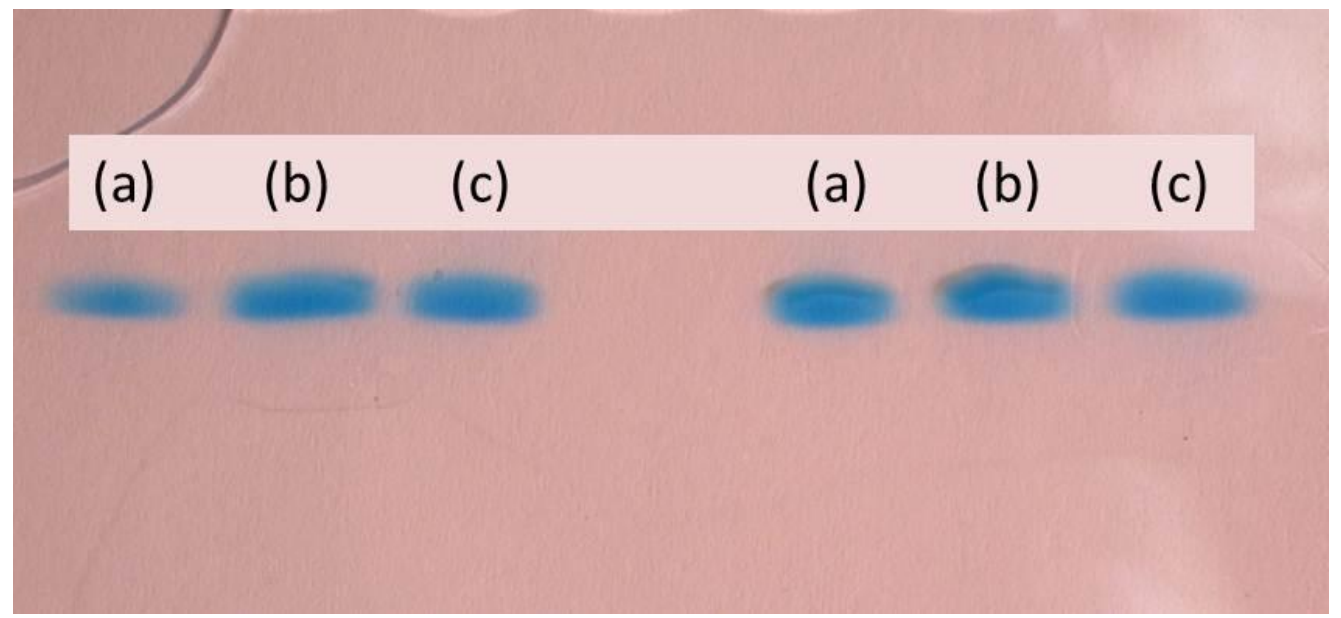

Figure 3-13. SDS-PAGE result for (a) leuprolide; (b) drug extracted from PLGA-ester micro particles; and (c) drug extracted from PLGA-acid micro particles.

Note: Samples were run in duplicate and results are presented as two separate sets. 

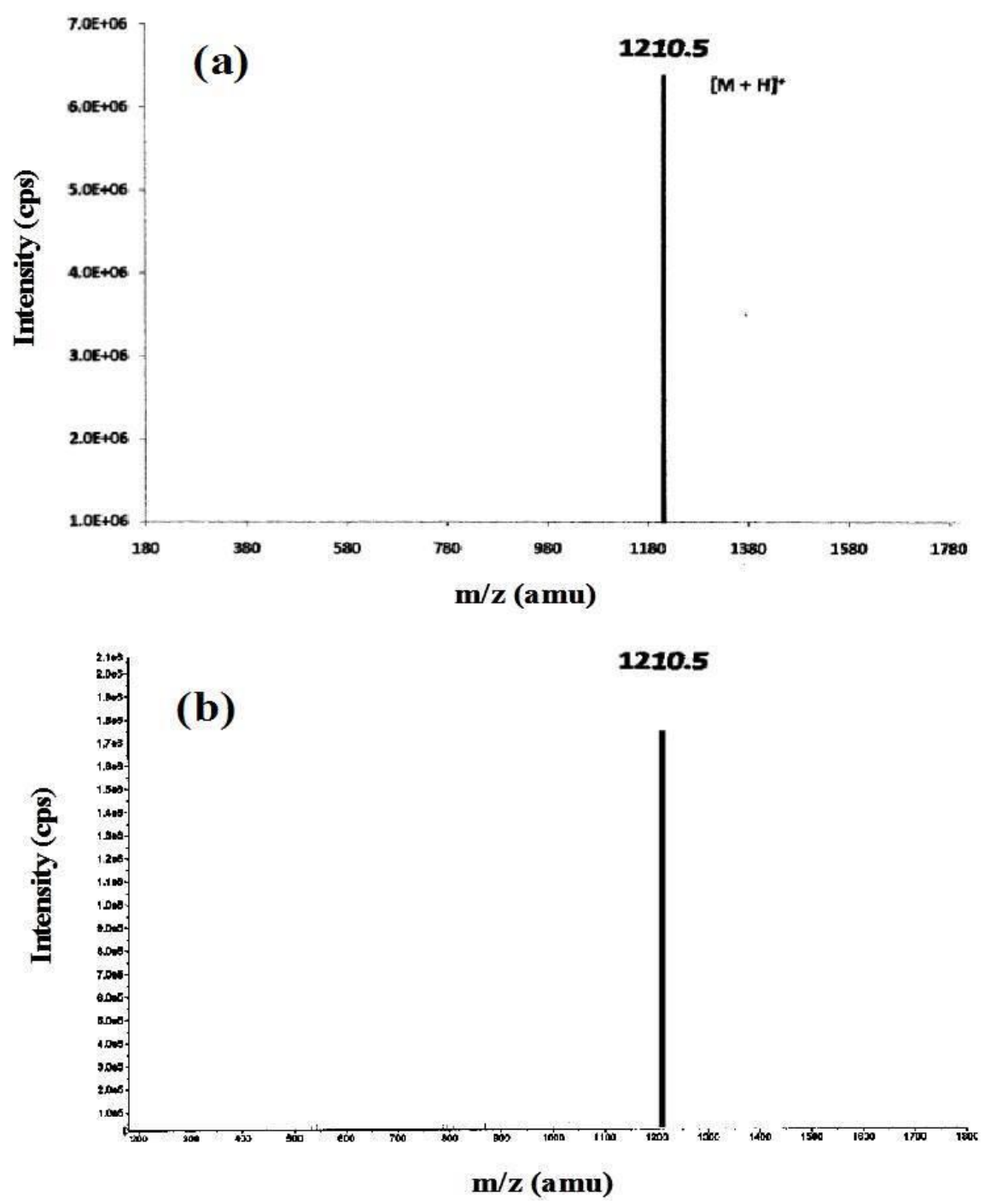

Figure 3-14. LC/MS chromatograms for (a) leuprolide; (b) drug extracted from PLGA-ester micro particles. 
interaction between polymer and solvent, we can essentially modulate the release rate of drug. Rate of dissolution was calculated for all the formulations and the values at 48 hours of dissolution were used for profile comparison. Figure 3-15 compares the change in Tg for three hydrophobic solvents/plasticizers i.e. TEC, ATEC and ATBC during in vitro release studies. Among these three plasticizers, only ATEC is able to maintain lowest Tg values for longest time periods. Figure 3-16, Figure 3-17 and Figure 3-18 shows the relationship between dissolution rate and $\mathrm{Tg}$ of the polymer mass as a function of dissolution time for formulations containing TEC, ATEC and ATBC respectively. Dissolution rate shows a negative correlation with $\mathrm{Tg}$ for formulations containing TEC and ATEC as hydrophobic solvents/plasticizers (Figure 3-16 and Figure 3-17). This can be explained by the relative hydrophilicity of PEG that results in faster release of PEG from the formulations. PEG leaves into the dissolution media resulting in an increase in the $\mathrm{Tg}$ of the remaining polymer mass. This is attributed to the formation of a relatively rigid polymer structure after PEG leached out into dissolution media. The effect becomes even more pronounced upon increasing the PEG content in the formulation. As PEG leaves from the formulations containing initially relatively higher amounts of PEG, the remaining polymer mass attains an even higher rigid structure.

ATBC formulations, on the other hand, show a positive correlation between dissolution rate and $\mathrm{Tg}$ of polymer mass (Figure 3-18). ATBC formulations on the other hand, exhibit an increase in dissolution rate with the increasing amount of PEG in the formulations. In this case, although, the fluidity of the polymer mass is increasing with higher amount of ATBC in the formulation, however, due to the very hydrophobic nature of ATBC, it doesn't allow water to penetrate into the polymer mass during dissolution thereby resulting in a decreased dissolution rate. Further, the Tg of the polymer mass remaining after 48 hours of dissolution was compared with the amount of PEG in the initial formulation. Figure 3-19 shows the surface response plots with Tg and gel thickness (or swelling factor) as the dependent variables and the type of hydrophobic plasticizer and percent PEG as the independent variables. It is clearly evident from the response plot that $\mathrm{Tg}$ of the polymer mass increases as a function of the amount of PEG in the formulation in presence of all different hydrophobic plasticizers i.e. TEC, ATEC, and ATBC. This can again be explained by the fact that the remaining polymer mass becomes rigid after relatively hydrophilic PEG leaves the formulation and diffuses into the dissolution media. Larger the amount of PEG in the formulation, higher is the overall amount of PEG leaving into the dissolution media resulting in an increase in $\mathrm{Tg}$ of the polymer mass left. Polymer gel thickness decreases for all the formulations as a function of the amount of PEG in the formulations due to a similar reason as explained above i.e. PEG leaves the polymer mass fairly easily, thereby decreasing the overall volume of the polymer depot in the dissolution media. Overall, as the PEG leaves out with respect to time during dissolution, there is a decrease in the swelling factor and a simultaneous increase in the Tg of polymer. It can therefore be concluded from this study that a larger ratio of the hydrophilic plasticizer to the hydrophobic plasticizer results in a slower drug release and lowers the overall drug dissolution rate.

PEG was further compared to NMP in terms of its ability to control release rate of 


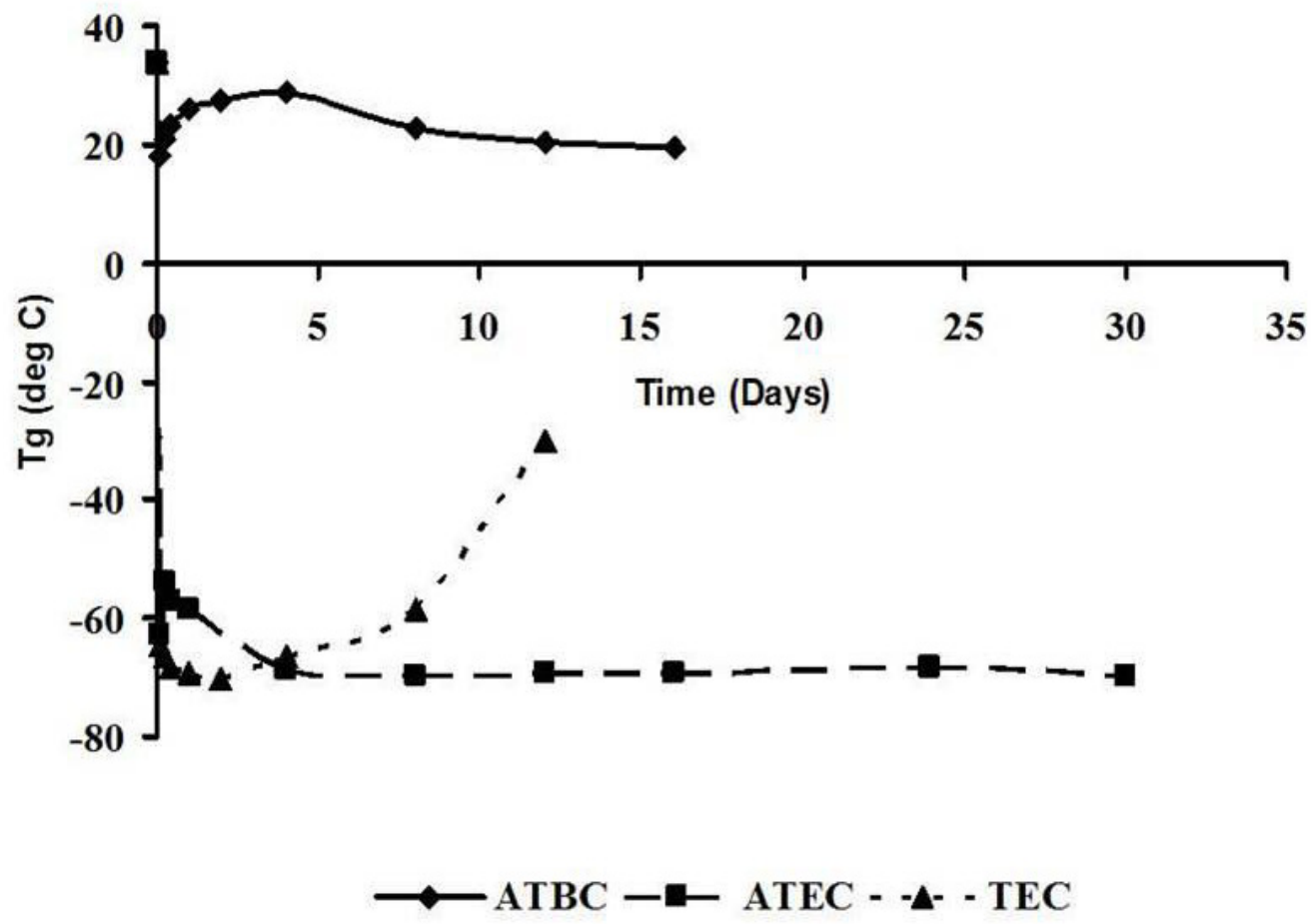

Figure 3-15. Representation of polymer $\mathrm{Tg}$ as a function of time during in vitro release study. 


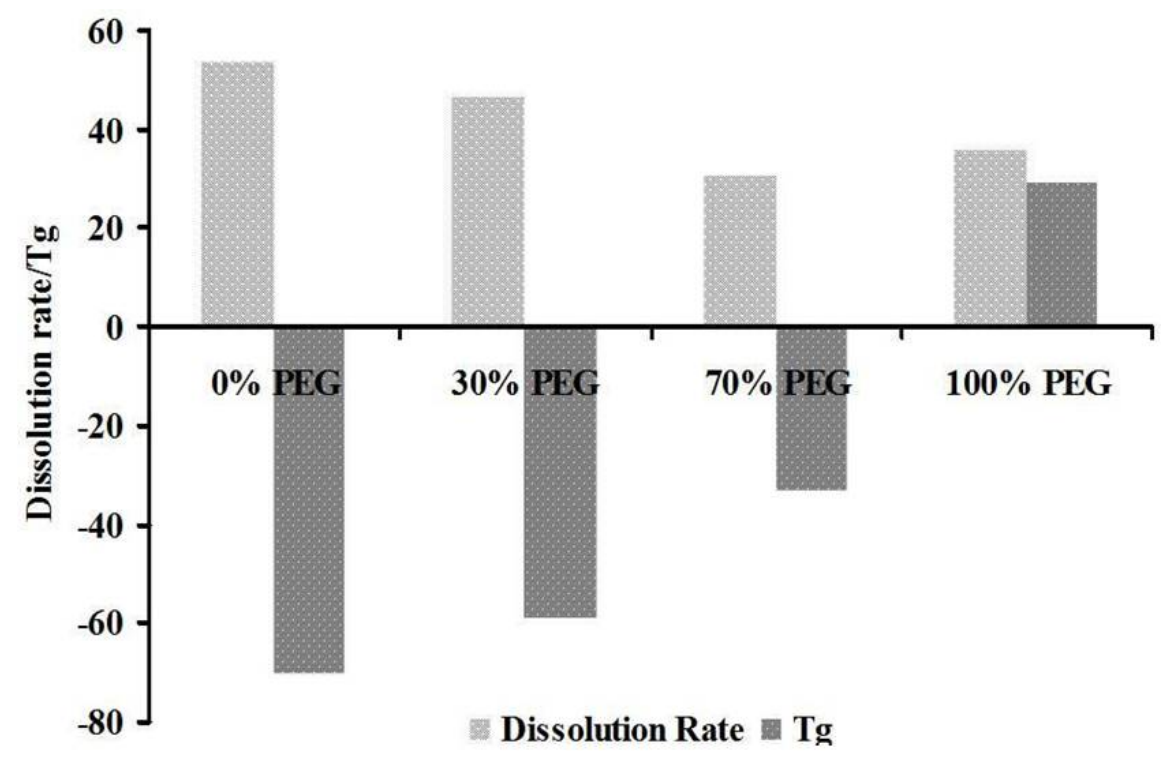

Figure 3-16. Comparion between dissolution rate and $\mathrm{Tg}$ as a function of percentage PEG amount in the plasticizer combination with TEC.

Note: Dissolution rate is expressed as percent drug dissolved per day and $\mathrm{Tg}$ is represented in deg $\mathrm{C}$. 


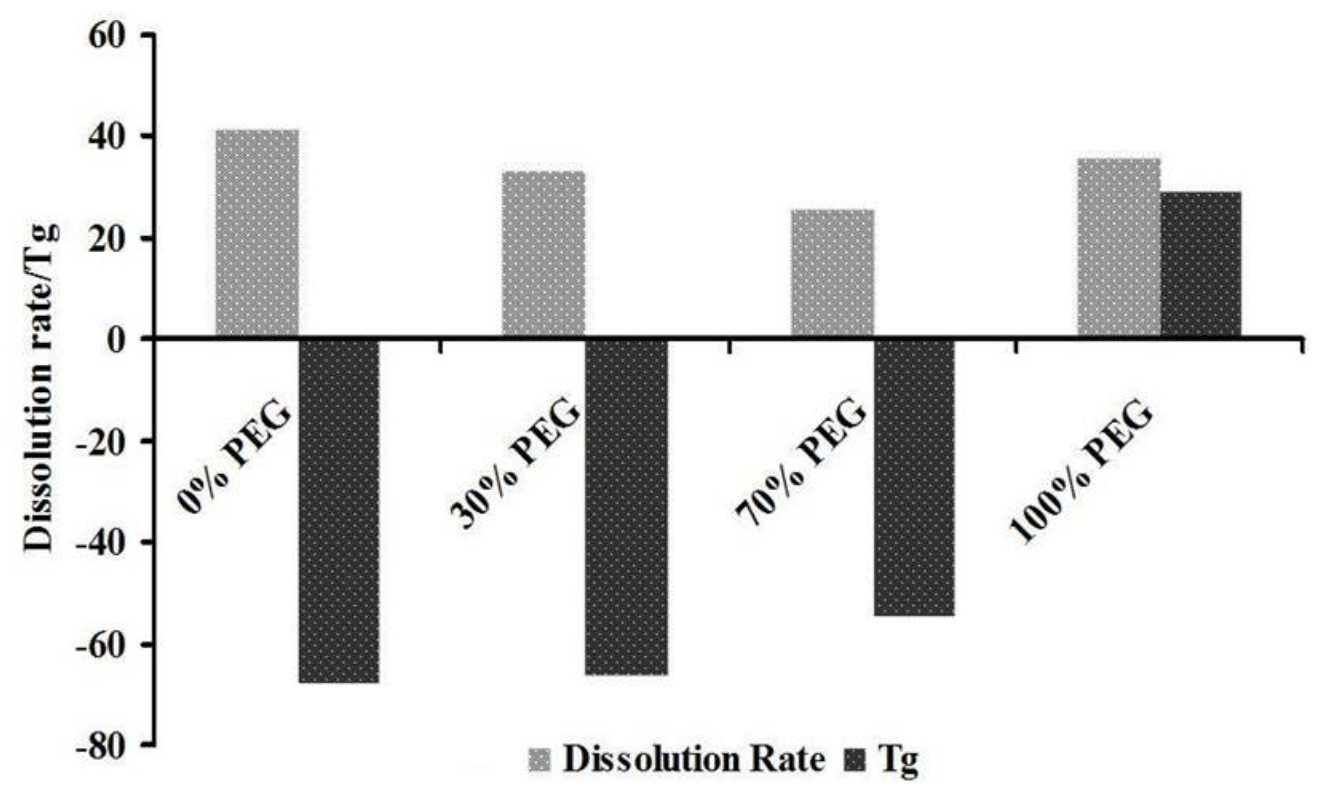

Figure 3-17. Comparison between dissolution rate and $\mathrm{Tg}$ as a function of percentage PEG amount in the plasticizer combination with ATEC.

Note: Dissolution rate is expressed as percent drug dissolved per day and $\mathrm{Tg}$ is represented in $\operatorname{deg} \mathrm{C}$. 


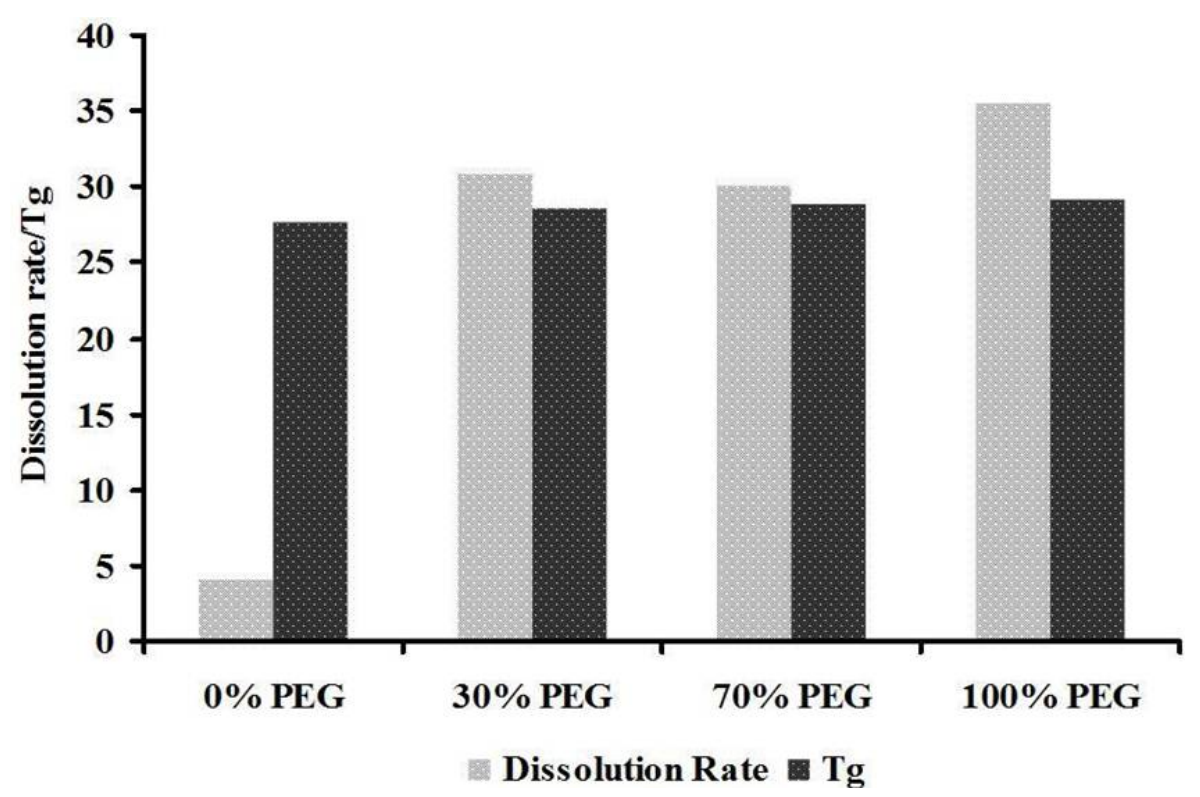

Figure 3-18. Comparison between dissolution rate and $\mathrm{Tg}$ as a function of percentage PEG amount in the plasticizer combination with ATBC.

Note: Dissolution rate is expressed as percent drug dissolved per day and $\mathrm{Tg}$ is represented in $\operatorname{deg} \mathrm{C}$. 

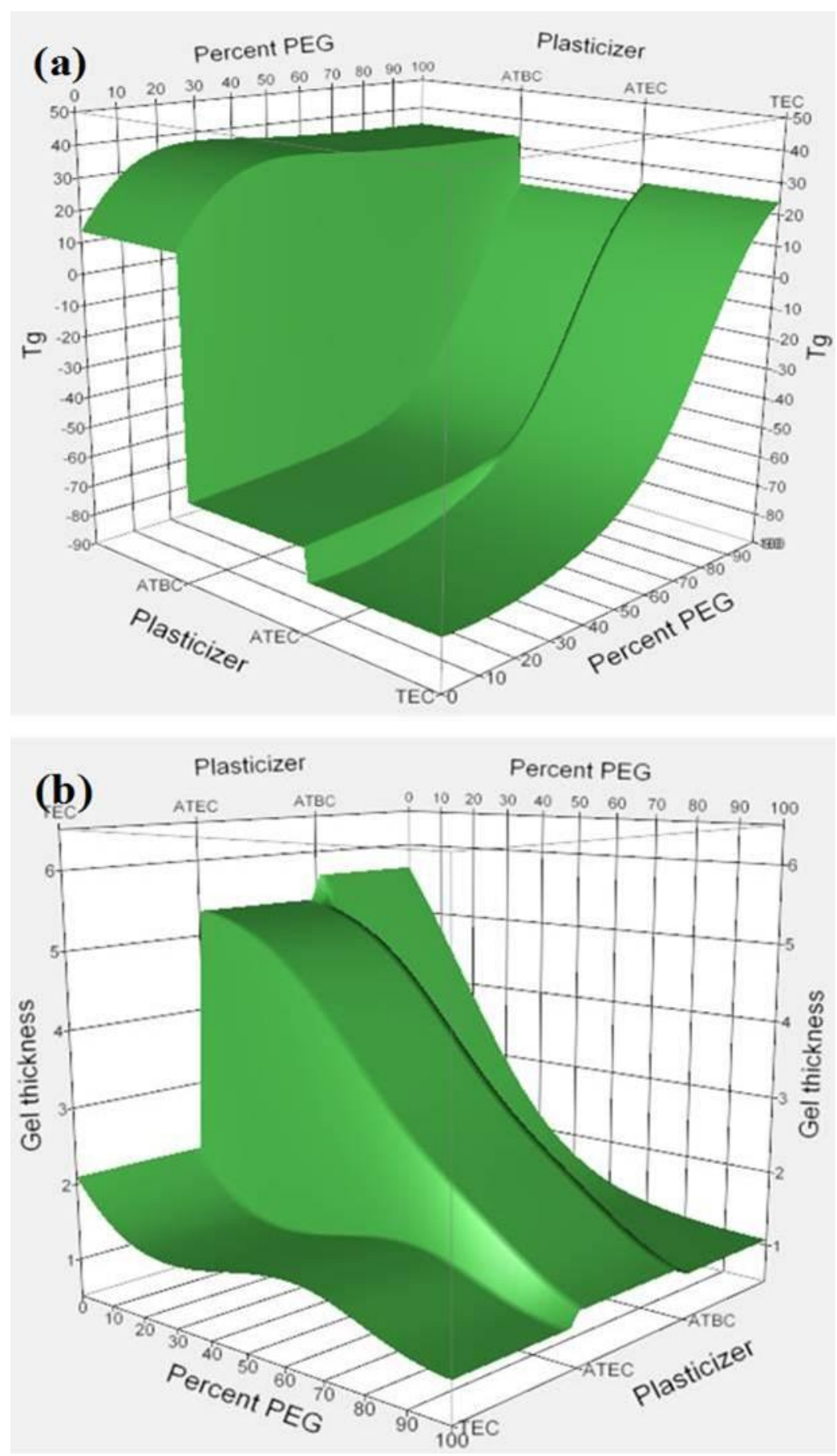

Figure 3-19. Response surface plots for (a) Tg; and (b) gel thickness as a function of plasticizer type and percent PEG in the plasticizer composition.

Notes: $\mathrm{Tg}$ is represented in $\operatorname{deg} \mathrm{C}$.

Gel thickness was measured with vernier calipers in $\mathrm{mm}$. 
the drug. Figure 3-20 shows a comparison of drug release from drug-polymer micro particles suspended in pure PEG and NMP solvents. It can be clearly seen from the results that PEG causes a higher burst effect than NMP, which could be attributed to relatively higher drug solubility in PEG than NMP. This difference in solubility could not be seen in the equilibrium solubility results since, the entire amount of drug went into solution in NMP and PEG thus not leaving behind any excess of drug. The drug release studies however clearly show the higher solubility and burst effect of drug with PEG than with NMP as the hydrophilic solvents.

\subsubsection{Selection of solvents/plasticizers for formulation development}

The results from drug solubility, stability and plasticizer screening studies were combined together to aid in selection of the solvents/plasticizers for further formulation development work. Since the idea was to select a solvent(s)/plasticizer(s) that can interact well with the polymer and keep it plasticized for longer times, ATEC was chosen as the solvent. To further strengthen the interaction between polymer and plasticizer (as seen by an initial greater drop in Tg of polymer in presence of NMP, Figure 3-21), a hydrophilic plasticizer was added to the system. NMP was selected over PEG as the hydrophilic solvent due to higher burst effects and therefore a greater potential of a biphasic or tri-phasic release profile of drug release observed with PEG solvent. However, one requirement is also to keep the drug solubility in the system to its minimum. Therefore, the minimum amount of NMP required in the solvent system was determined by visual comparison of the polymer gelling over a period of first 24 hours (Figure 3-22). $0.5 \%$ $\mathrm{w} / \mathrm{w}$ NMP in the plasticizer composition resulted in polymer gelling in 10 hours whereas increasing the NMP amount to $10 \% \mathrm{w} / \mathrm{w}$ accelerated the gelling phenomenon significantly causing complete polymer gelation within 3 hours. 10\% w/w NMP was therefore selected in combination with ATEC as the optimal plasticizer composition for further evaluation of other formulation factors.

\subsection{Conclusion}

Optimum plasticizer composition was developed for drug loaded PLGA micro particles for the purposes of drug delivery. Based on drug and polymer solubility in various plasticizers, molecular interaction between polymer and plasticizer and initial burst release of drug, a plasticizer composition containing NMP and ATEC with a volume proportion of 1:9 w/w was most appropriate for diffusion controlled drug release purposes. PLGA polymer matrix provided the desired stabilizing conditions for the drug by preventing peptide aggregation at high temperatures. PLGA with acid end group interacted with the positively charged amino group in the drug molecule thereby solubilizing the drug in polymer matrix and promising a further reduction in burst release of drug and a more continuous drug delivery. 


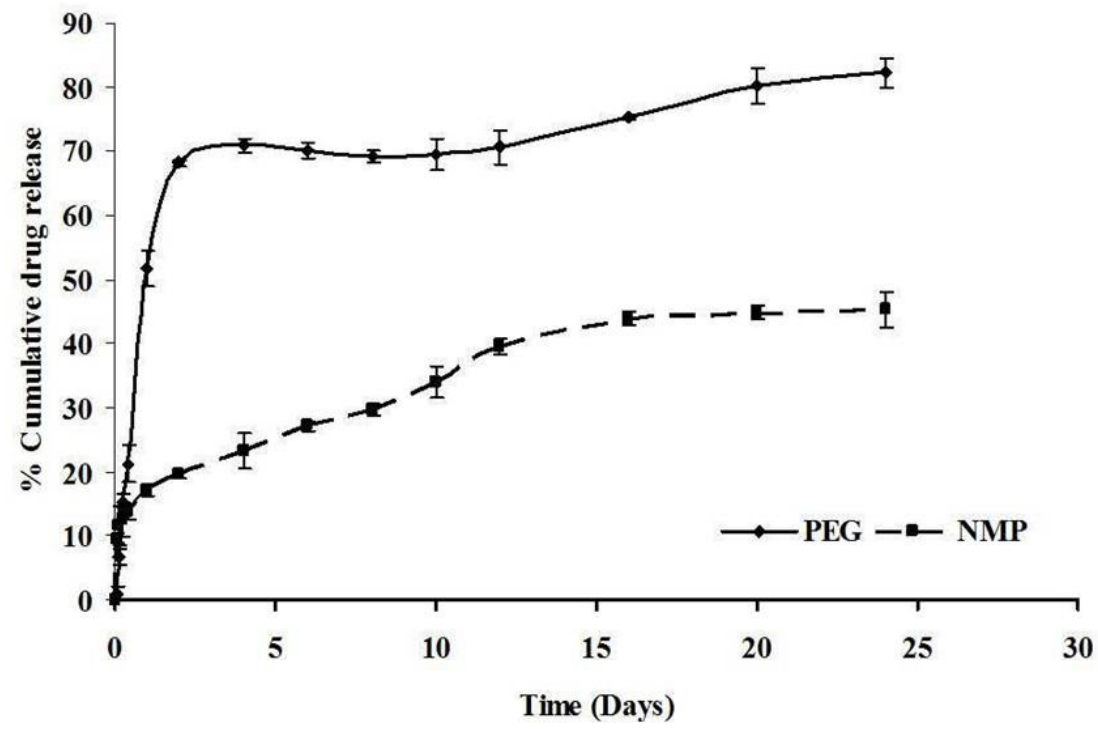

Figure 3-20. Leuprolide release from PLGA micro particles as a function of time in presence of PEG and NMP.

Notes: Each data point represents an average of three measurements.

Standard deviation of three measurements is presented as error bars. 


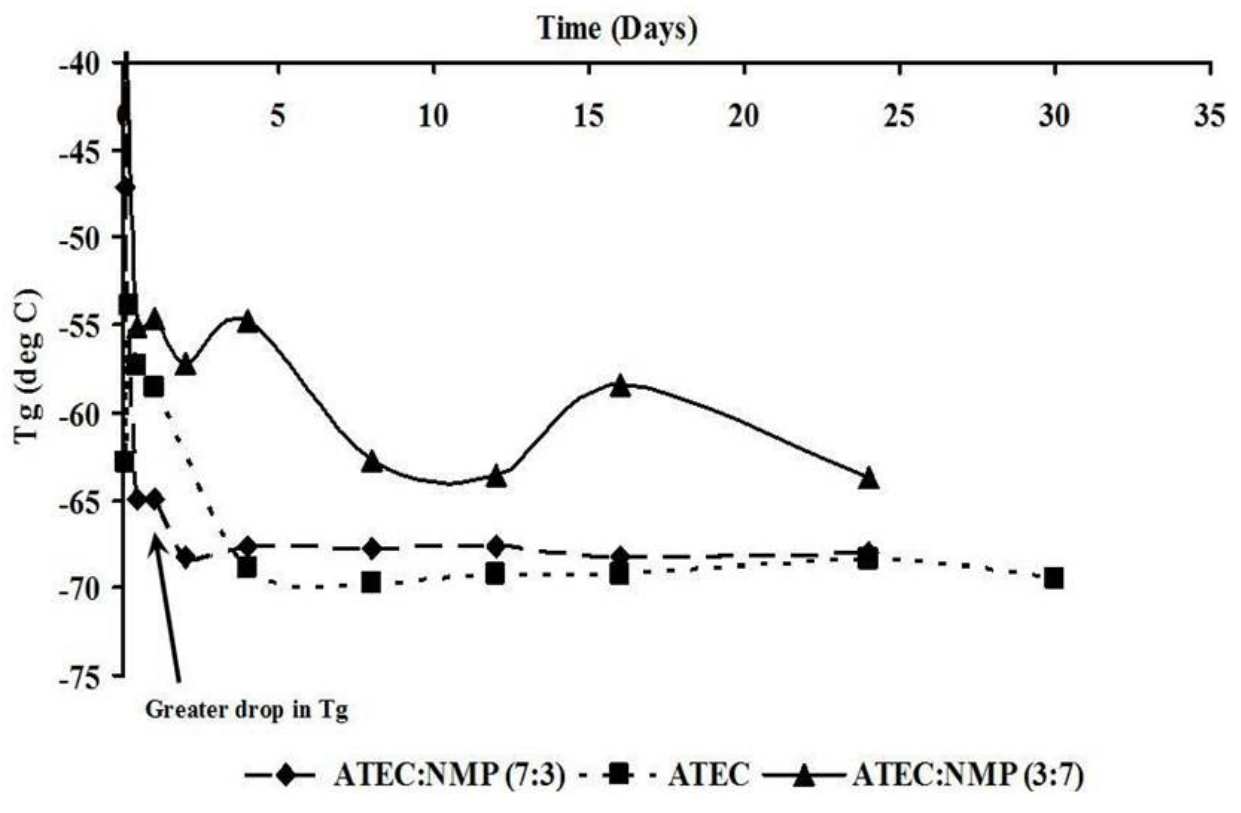

Figure 3-21. Drop in Tg as a function of NMP concentration in the plasticizer composition.

Note: Each data point represents individual polymer gel samples at specific time points. 


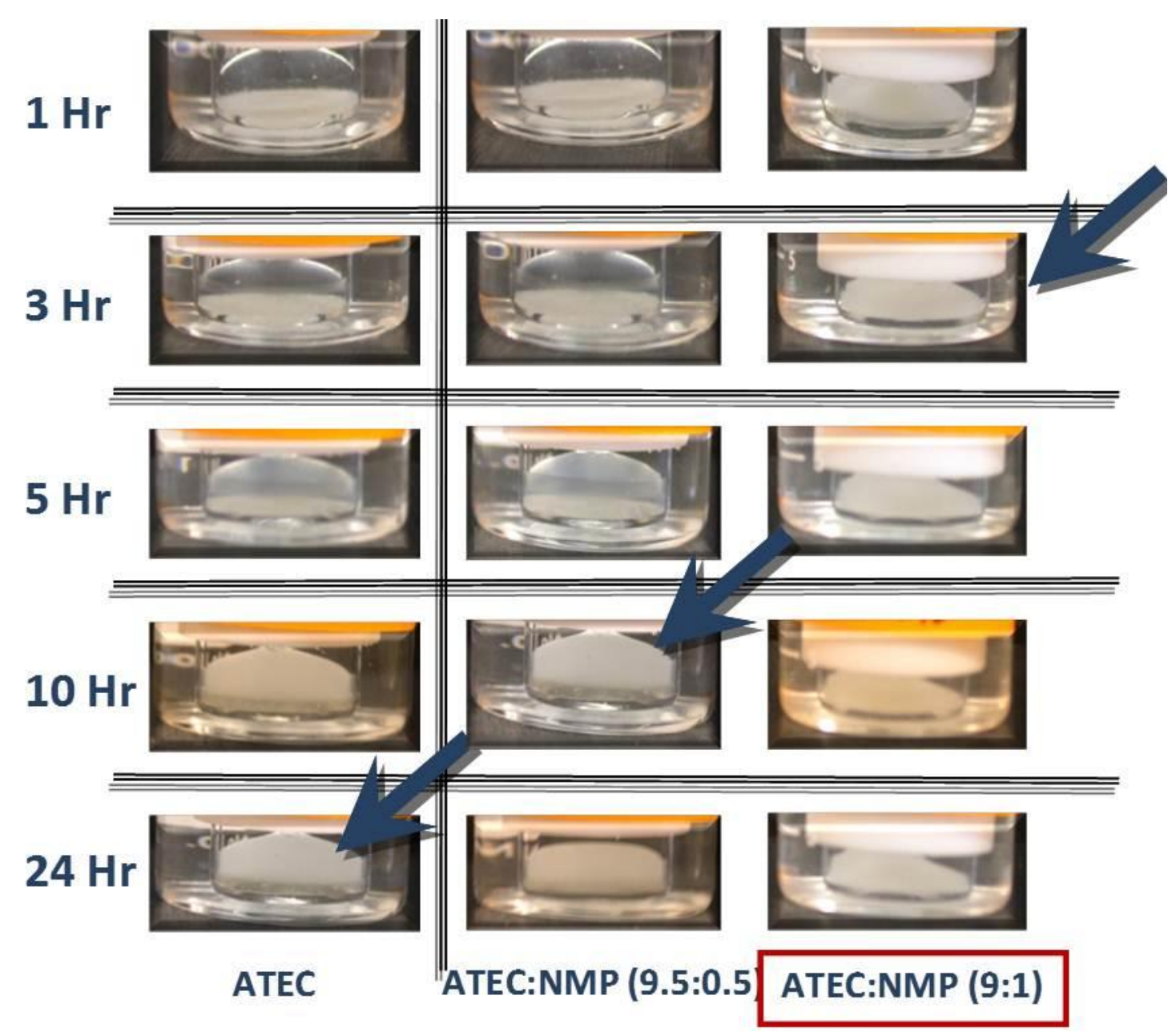

Figure 3-22. Polymer gelling in presence of increasing concentrations of NMP as a function of time.

Note: Polymer gelling was much faster and was complete in 3 hours in presence of $10 \%$ $\mathrm{w} / \mathrm{w}$ of NMP in the plasticizer composition. 


\section{CHAPTER 4. DEVELOPMENT AND CHARACTERIZATION OF AN IN SITU GELLING PLGA MICRO PARTICLE BASED IMPLANT}

\subsection{Introduction}

PLGA (poly lactide-co-glycolide) polymer is a well-known biodegradable and biocompatible polymer for injectable controlled drug delivery applications. Various actives have been formulated till date using this polymer and are available on market. These include Lupron ${ }^{\circledR}$ depot containing leuprorelin, Trelstar ${ }^{\circledR}$ depot containing triptorelin, Sandostatin ${ }^{\circledR}$ depot containing octerotide and Nutropin ${ }^{\circledR}$ depot containing human growth hormone to name a few. Most of these systems are formulated as microspheres using PLGA polymer. Apart from the microsphere systems, depot systems based on polymer solutions are also well studied. ${ }^{112,113}$ One such system is Eligard ${ }^{\circledR}$ based on Atrix technology of in situ solvent removal and polymer precipitation, where the solvent used is N-methyl pyrrolidone (NMP) and polymer is PLGA. ${ }^{114}$ Nonetheless, majority of the currently formulated PLGA based delivery systems result in a characteristic tri-phasic release of the drug. ${ }^{115-117}$ This includes an initial burst release phase, a second plateau phase and a final erosion phase. The tri-phasic release profile is not desirable for several different reasons i.e. the initial burst release might result in toxic drug concentrations in the body, the plateau phase might result in drug concentrations that are much below the therapeutic level of drug and the final erosion phase might lead to a second burst release of drug. Moreover, a high viscosity of some of these PLGA solution based implants might lead to painful injections. ${ }^{118}$

In order to effectively apply the concept of drug delivery with polymers such as PLGA, it is necessary to have a controlled rate of hydrolytic degradation of this polymer. One of the factors that work against the constant drug release from PLGA based delivery systems is its tendency to undergo bulk degradation phenomenon. ${ }^{117}$ PLGA polymer therefore does not allow for a continuous controlled release of drug without any external intervention in the delivery system. A diffusion controlled drug release is much desired over an erosion controlled release due to several different reasons. Most importantly, it not only results in a more continuous release of the drug but also of the acidic degradation products of the PLGA polymer i.e. lactic acid and glycolic acid. Such a continuous diffusion of the polymer degradation products from the depot system prevents the development of an acidic $\mathrm{pH}$ in the microenvironment. This enhances the possibilities of encapsulating most of the peptide, polypeptide and protein based drugs in PLGA polymeric delivery systems without compromising the structural stability of such drugs. An extensive work has been done on modifying the bulk eroding properties of the PLGA polymer and imparting a surface erosion element to polymer by e beam irradiation. ${ }^{119}$

Furthermore, numerous approaches pertaining to modification of PLGA microsphere structure and compositional changes in PLGA based delivery systems have been explored till date with the objective of overcoming the tri-phasic release pattern inherent in the PLGA based delivery systems. Various formulation strategies are being currently introduced that can potentially make the drug release from the PLGA based 
systems more diffusion controlled.

These include addition of release rate modifying agents (e.g. medium chain triglycerides, glycerol etc.) into the microsphere structure, ${ }^{120}$ modifying the microsphere preparation process or even modifying the PLGA polymer backbone. ${ }^{121}$ Although able to provide a more continuous drug release, most of the current techniques are much complicated and are limited by the fact that the drug release is still erosion controlled to a certain extent.

For the purpose of present research work, an ideal in situ polymer gelling system is defined as the one, whereby the solid polymer particles interacts with the plasticizer molecules resulting in polymer chain relaxation. During this time, the rate of interaction of plasticizer with the polymer is greater than its affinity towards the surrounding environment. As a result, the polymer particles first undergo gelation before finally the plasticizer leaches out leaving the precipitated polymer behind. Further, the rate of plasticizer release is much more controlled thereby preventing sudden polymer precipitation at any point. This essentially serves a dual purpose: first the viscosity of formulation is lower compared to a polymer solution based system thereby resulting in lower injection forces and provides ease of injectability and second the drug undergoes a diffusion controlled release process due to sufficiently and uniformly plasticizer polymer. One of the crucial aspects that should be paid due attention to is the physical stability of such in situ gelling polymer suspension systems. It is important that the plasticizer although possess great affinity for the polymer, does not interact or plasticizer the polymer significantly within the syringe prior to administration as it would otherwise lead to serious loss of injectability. A practical way of introducing physical stability into the formulation system is to incorporate a polymer immiscible component into the injection vehicle. The polymer immiscible component is completely miscible with the plasticizer and significantly dampens the polymer plasticizer interaction prior to administration. Once administered, the polymer immiscible component forms a separate phase, thereby allowing complete interaction between polymer and plasticizer. One such component that exhibits the ability of both miscibility with the plasticizer as well as forming a separate phase upon equilibration with excess water is glyceryl monooleate (GMO). GMO has been known to form various liquid crystalline structures once equilibrated with excess water. $^{122}$ The cubic liquid crystalline structure of GMO has been studied to reduce burst release of an oligonucleotide from PLGA micro particles by dispersing the drug loaded micro particles in an in situ cubic phase carrier. ${ }^{123}$ Hydrophilic cosolvents such as polyethylene glycol (PEG 300), propylene glycol (PG) and ethanol were used along with GMO to constitute an in situ cubic phase forming carrier. The cosolvents being extremely hydrophilic leaves immediately into the surrounding media resulting in a matrix formation consisting of micro particles embedded in the cubic phase.

The objective of this study was to simplify drug delivery from PLGA polymer by developing a novel injection vehicle system that is capable of modulating the drug release from the PLGA polymeric system (micro particles or microspheres) in a more diffusion controlled manner. The injection vehicle consists of a relatively hydrophobic polymer plasticizer(s) that allows for polymer chain relaxation and drug diffusion. The second 
important component of the injection vehicle is the polymer immiscible component that provides the necessary physical stability to the suspension system prior to administration. In this study, we evaluated the system for its ability to release the drug in a diffusion controlled manner and for the physical stability of the suspension system prior to administration by measuring the injection forces.

\subsection{Materials and Methods}

\subsubsection{Materials}

PLGA 50:50 (IV 0.59dL/g) was obtained from Lactel absorbable polymers, Durect Corporation, Pelham, AL, USA. Glyceryl monoleate (Capmul GMO-50, EP/NF) was used as the glycerol lipid and was obtained as a generous gift from Abitec Corp., Janesville, WI, USA. NMP (N-methyl pyrrolidone; Pharmasolve), which is the hydrophilic solvent was procured from ISP Pharma Technologies, Wayne, NJ, USA and ATEC (acetyl triethyl citrate), which is the hydrophobic solvent was obtained from Morflex Inc., Greensboro, NC, USA. PEG 1450 (CarbowaxTM) and HPMC E15 PREM LV were obtained from The Dow Chemical Co., Midland, MI, USA. Leuprolide acetate was used as the model drug and was purchased from Teva Pharmaceuticals, Israel. All buffers were prepared in reverse osmosis water. Other chemicals and reagents were purchased from Sigma Aldrich.

\subsubsection{Methods}

\subsubsection{Preparation of micro particles by film grinding method}

PLGA polymer was dissolved in acetone in a ratio of $1: 10 \mathrm{w} / \mathrm{w}$ of polymer to solvent under constant magnetic stirring at room temperature. Leuprolide acetate $(16 \%$ $\mathrm{w} / \mathrm{w}$ of polymer weight) was dispersed in the polymer solution. Any large drug particles were broken by brief sonication in a water bath. The drug was allowed to finely disperse in the polymer solution under constant magnetic stirring for 30 minutes. The solvent was then allowed to evaporate in a fume hood until almost the entire solvent is evaporated leaving a flow able polymer solution behind. The flow able polymer solution containing finely dispersed drug thus obtained was transferred to a porcelain petri dish that was transferred to a vacuum oven for further drying of the polymer mass. The drug containing polymer mass was dried for 48 hours at $40^{\circ} \mathrm{C}$ at $25 \mathrm{in} \mathrm{Hg}$ of vacuum.

The dried polymer film was scrapped off the petri dish followed by grinding into fine particles using a cryomill. The film was transferred into a stainless steel cryomill jar, which was completely submerged into liquid nitrogen. The jar was taken out after the liquid nitrogen stops bubbling. The film was ground at a frequency of $24 \mathrm{~Hz}$ for 4 minutes. The particles thus obtained were passed through a 120 mesh screen to remove 
any larger particles. Figure 2-2 (Chapter 2) shows the schematic of micro particle preparation by film grinding method.

\subsubsection{Preparation of microspheres by modified $W / O / W$ multiple emulsion method}

A primary $\mathrm{W} / \mathrm{O}$ emulsion was prepared by adding $0.25 \mathrm{ml}$ of an aqueous phase containing leuprolide acetate and 50\% w/w PEG 1450 (polyethylene glycol) to $2.5 \mathrm{ml}$ of an organic phase containing dichloromethane and acetone in a volume ratio of $4: 1$. The emulsion thus formed was immediately subjected to probe sonication in brief pulses of 10 seconds each. The primary emulsion was then kept at $-20^{\circ} \mathrm{C}$ for 5 minutes. This was followed by adding the primary emulsion to the external aqueous phase $(0.01 \mathrm{M}$ phosphate buffer saline, PBS) containing 1.5\% HPMC E15 PREM LV (pH adjusted to 10.5 with $\mathrm{NaOH}$ ) as the thickening agent with the entire set up placed on an ice bath. The organic solvent was evaporated using a rotary evaporation for 10 minutes followed by addition of $35 \mathrm{ml}$ of PBS and further evaporation for next 10 minutes. The particles thus obtained were filtered through 0.22 micron nylon filter using a vacuum filtration assembly. After drying at $40^{\circ} \mathrm{C}$ under vacuum for 2 hours, the dried particles were scrapped off the filter and passed through 100 mesh screen. The schematic of microsphere preparation by the modified $\mathrm{W} / \mathrm{O} / \mathrm{W}$ multiple emulsion method is depicted in Figure 4-1.

\subsubsection{Preparation of PLGA solution formulation}

PLGA polymer was dissolved under gentle heat in the plasticizer combination of ATEC and NMP $(9: 1 \mathrm{w} / \mathrm{w})$ at 14\% w/w loading. The drug was dispersed in the polymer solution immediately before in vitro drug release testing.

\subsubsection{Assay of drug in PLGA micro particles/microspheres}

The drug content in PLGA micro particles/microspheres was determined by solvent extraction procedure. The particles were dissolved in $5 \mathrm{ml}$ of dichloromethane followed by extraction of drug in $10 \mathrm{ml}$ water under constant stirring. The drug content was analyzed by a RP-HPLC method (Chapter 2).

\subsubsection{Construction of phase diagrams for the binary/ternary solvent based system}

Phase diagrams were constructed using PLGA, plasticizer(s) and the glycerol lipid as the three individual components. PLGA was dissolved in different quantities of the polymer plasticizer(s) and the polymer was forced to precipitate upon addition of the anti-solvent (glycerol lipid) in the polymer solution. Glyceryl monoleate being immiscible with the PLGA polymer causes the polymer precipitation which was captured as the end point. 


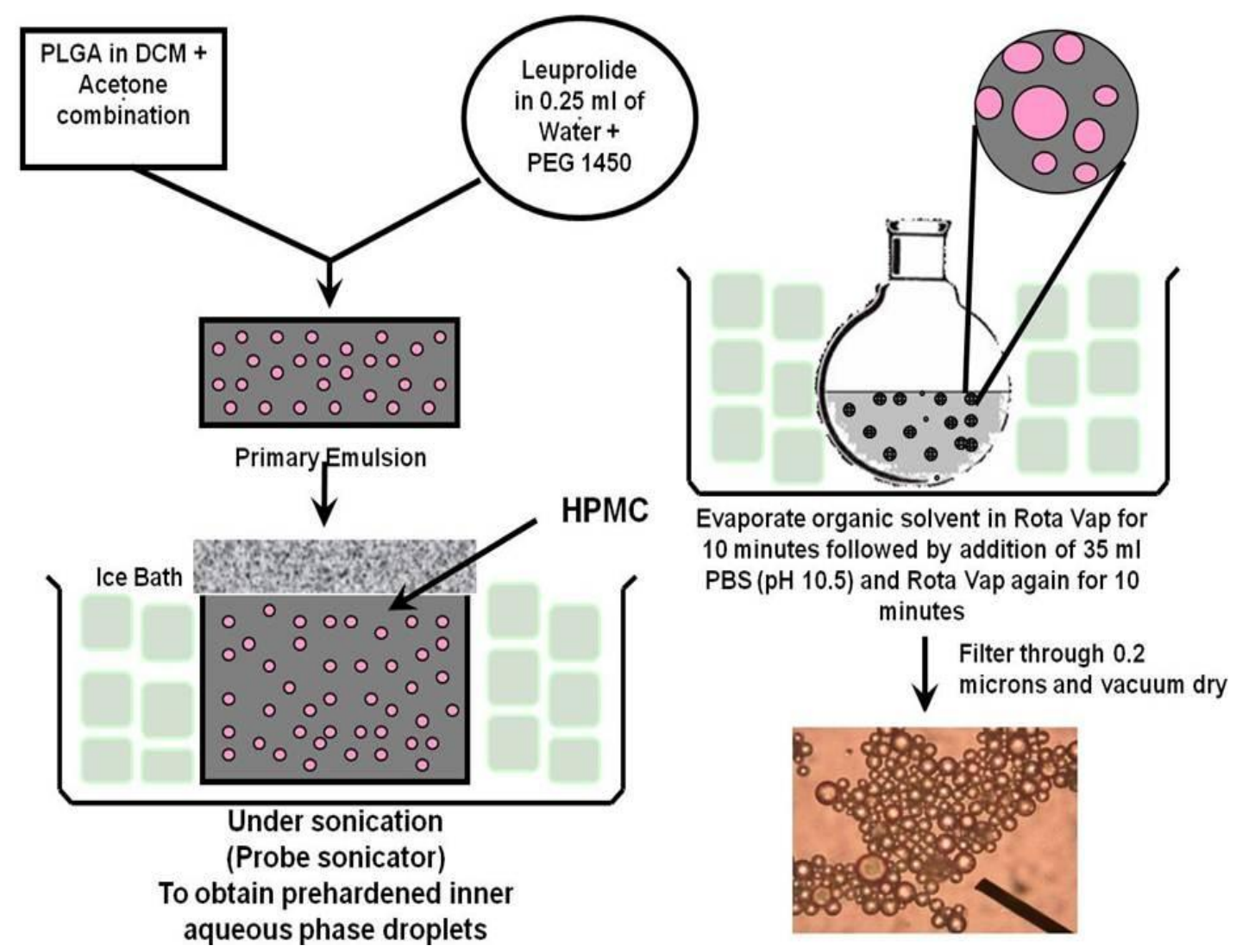

Figure 4-1. Schematic representation of PLGA microsphere preparation by modified multiple emulsion method. 
The results were plotted as a ternary phase diagram. NMP/ATEC $(1: 9 \mathrm{w} / \mathrm{w})$ represented the binary solvent and NMP/ATEC/EB $(1: 4.5: 4.5 \mathrm{w} / \mathrm{w} / \mathrm{w})$ represented the ternary solvent system for the purpose of constructing the phase diagrams.

\subsubsection{Light microscopy}

Light microscopy was performed on PLGA micro particles/microspheres using a Nikon Microphot FX microscope. The images were captured with a high resolution Kodak DCS 460 color digital camera mounted on the microscope. Using a PowerPC G4, images were viewed on an Apple 20" Cinema Display monitor.

\subsubsection{Polarized light microscopy}

Polarized light microscopy was conducted on the injection vehicle system after equilibration with excess water or dissolution media to understand the crystalline structures formed by glycerol lipid. A Nikon Microphot FX microscope equipped with a polarizing filter. The images were captured with a high resolution Kodak DCS 460 color digital camera mounted on the microscope. Using a PowerPC G4, images were viewed on an Apple 20" Cinema Display monitor.

\subsubsection{MDSC (Modulated differential scanning calorimetry) studies}

MDSC studies on the injection vehicle system were conducted after equilibration with excess water by using a Thermal Analysis (TA) Q 2000 series DSC instrument with autosampler. The parameters selected for the scan were: scanning rate of $3^{\circ} \mathrm{C} / \mathrm{min}$, modulation temperature amplitude of $\pm 1.00^{\circ} \mathrm{C}$ and sinusoidal modulation of $60 \mathrm{sec}$.

\subsubsection{In vitro drug release studies}

Drug release was conducted in $0.012 \mathrm{M}$ PBS (pH 7.4) containing $0.02 \%$ sodium azide. $10 \mathrm{ml}$ of dissolution media was taken in a $20 \mathrm{ml}$ glass scintillation vial. Vials were kept in a shaker incubator maintained at $37^{\circ} \mathrm{C}$ and $100 \mathrm{rpm}$ rotation. Formulations were placed at the bottom of glass vials and $2 \mathrm{ml}$ aliquots were taken at specific time points with replacement using fresh buffer. Drug content was determined using a RP-HPLC method (Chapter 2).

\subsubsection{External and micro environmental pH measurement}

$\mathrm{pH}$ of the dissolution media was measured at specific time points during drug release studies using a Orion (Model $520 \mathrm{~A}$ ) $\mathrm{pH}$ meter. Micro environmental $\mathrm{pH}$ was

measured by confocal laser scanning microscopy. A Bio-Rad MRC 1024 imaging system 
equipped with a Krypton/Argon laser and an upright Olympus BX50 microscope was used for the study. Fluorescein and Oregon green were selected as $\mathrm{pH}$ indicating fluorescent dyes with the pKa values of 6.4 and 4.8 respectively. Images were captured at 20x magnification at a box resolution of 1024 x 1024 pixels. A single green fluorophore scanning mode with a krypton/argon laser was used at an excitation wavelength of 488 $\mathrm{nm}$. The detector parameters were: iris $2.50 \mathrm{~mm}$, gain 1220.00 , and black 10.00. Standard curves were constructed with fluorescent intensity as the output parameter w.r.t. $\mathrm{pH}$ of the dissolution media. Fluorescein and Oregon green were added at concentrations of $0.0054 \mathrm{mg} / \mathrm{ml}$ and $0.0045 \mathrm{mg} / \mathrm{ml}$ respectively to the dissolution media separately. One formulation containing dissolution glass vial was removed from the shaker incubator at every time point for both dyes and subjected to image analysis by confocal microscopy. The micro environmental $\mathrm{pH}$ was determined by measuring the fluorescent intensity and calculating the corresponding $\mathrm{pH}$ using the standard curves.

\subsubsection{Mathematical modeling of dissolution profiles}

Seven different models were used to determine the drug release mechanism from the PLGA micro particulate system in presence of the unique injection vehicle. An excel

add-in program, "DD Solver" was used for this purpose and $\mathrm{R}^{2}$ values were compared for different models.

\subsubsection{Microscopic examination of the structural changes in polymer depot}

A portion of formulation gel was withdrawn from the dissolution vials at specific time points at 7, 14, 21 and 32 days and examined for pore formation and other structural changes under an optical microscope (Nikon Microphot FX) at 20x optical zoom and images were captured using high resolution Kodak DCS 460 color digital camera.

\subsubsection{Viscosity and injection force measurement}

Viscosity of the injection vehicle with and without suspended PLGA micro particles and of the PLGA solution formulation was measured using Brookfield DV- III Ultra programmable cone and plate rheometer and Rheocalc 3.2 software. Two different cones of $2.4 \mathrm{~cm}$ diameter $/ 0.8$ degrees angle and $1.2 \mathrm{~cm}$ diameter $/ 3.0$ degrees angle were used for low and high viscosity systems respectively. Shear rate was varied between 0 and 300/sec and corresponding shear stress was recorded. Injection forces were determined using a Chatillon TCD-200 digital force tester connected to a computer and controlled by NEXYGEN FM series software A $3 \mathrm{ml}$ syringe was filled with $2 \mathrm{ml}$ of the formulation under test connected with a 19 gauge needle having 1 in length. The syringe was placed against the cylindrical probe of the load cell $(100 \mathrm{~N})$ and the plunger was pushed down under the compression mode of the load cell until the plunger was displaced from $2 \mathrm{ml}$ to $1 \mathrm{ml}$ mark on the syringe. The maximum injection force was recorded for 3 measurements per sample. 


\subsection{Results and Discussions}

\subsubsection{Comparison of micro particle morphology from two different methods}

Figure 2-2 (Chapter 2) and 4-1 shows the schematic of micro particle preparation using film grinding method and modified $\mathrm{W} / \mathrm{O} / \mathrm{W}$ multiple emulsion method. The microscopic images are depicted in Figure 4-2 and clear difference in the shape and morphology of micro particles can be observed. The film ground micro particles presented an irregular surface along with a high percentage of surface free drug (Figure 4-3) compared to the multiple emulsion microspheres, which had less than $3 \% \mathrm{w} / \mathrm{w}$ of surface free drug. The multiple emulsion microspheres have a relatively more uniform and spherical geometry with the morphology resembling that of microcapsules or even a honeycomb like structure.

\subsubsection{Effect of plasticizer and GMO ratio on drug release}

Three different vehicle compositions representing different plasticizer: GMO ratios were selected from the ternary phase diagram (Figure 4-4 (a)). The area on the left of tie line represents a stable suspension based system of PLGA micro particles in the injection vehicle. This essentially indicated that the polymer micro particles would not gel immediately upon dispersing into the injection vehicle thereby allowing time prior to administration. The region on the right of the tie line however represents a solution system, whereby the polymer micro particles would gel instantly thus causing serious injectability issues. The desired composition of injection vehicle is therefore selected from the region on the left of the tie line. The in vitro drug release from film ground PLGA micro particles is depicted in Figure 4-5. It can be clearly seen from the figure that a higher percentage of plasticizer(s) in the injection vehicle resulted in a relatively faster drug release which can possibly be explained by a greater polymer plasticization. Effect of PLGA polymer plasticization on drug release has been previously studied by Wang and co-workers where they observed an accelerated drug release by addition of PEG as a plasticizer to the PLGA polymer. ${ }^{124}$ The drug release exhibited a tri-phasic pattern at lower plasticizer amounts, whereas, the drug release gradually became more continuous upon increasing the amount of plasticizer. This indicates a more diffusion controlled drug release at higher plasticizer amounts. Further, a complete drug release was obtained from the in situ implant formulations containing relatively higher plasticizer amounts and there was almost no drug lost in the polymer matrix. The drug release from the multiple emulsion microspheres was also compared with that obtained from the film ground micro particles. Figure 4-6 shows the drug release from the two micro particle types in presence of an injection vehicle containing $50 \% \mathrm{w} / \mathrm{w}$ of plasticizer(s). The multiple emulsion microspheres exhibited an almost linear drug release throughout with minimal burst effect, which can be attributed to the total surface associated free drug with the micro particles/microspheres (Figure 4-3).After the initial burst phase, however, the formulations based on two different micro particle types released the drug in an almost continuous linear fashion. This essentially indicates that use of relatively more 

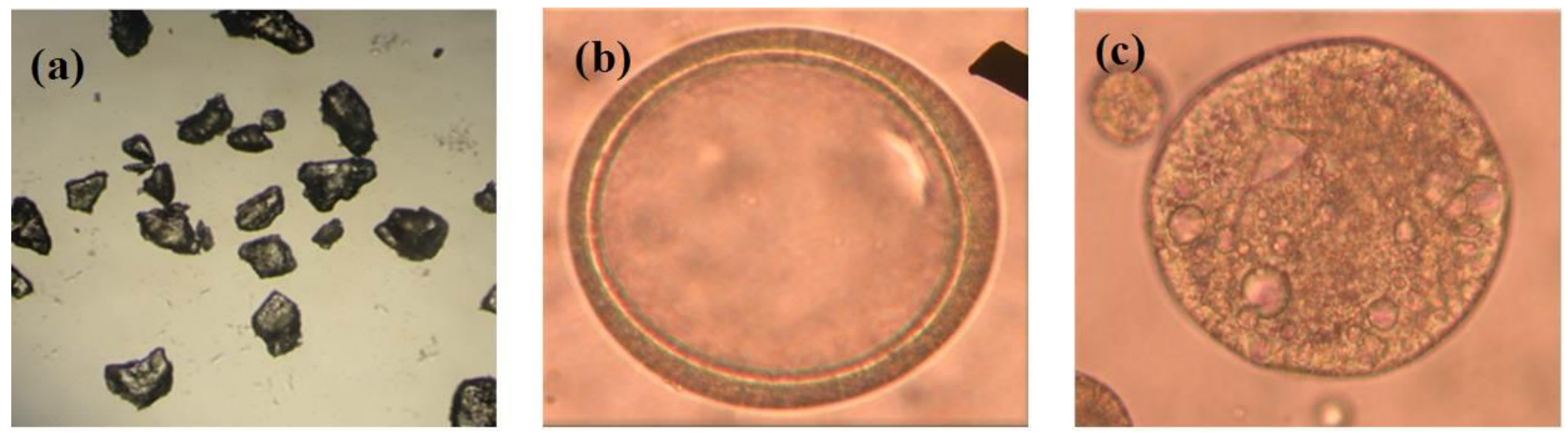

Figure 4-2. Microscopic images of PLGA micro particles prepared by (a) film grinding method; (b) and (c) W/O/W modified multiple emulsion method. 


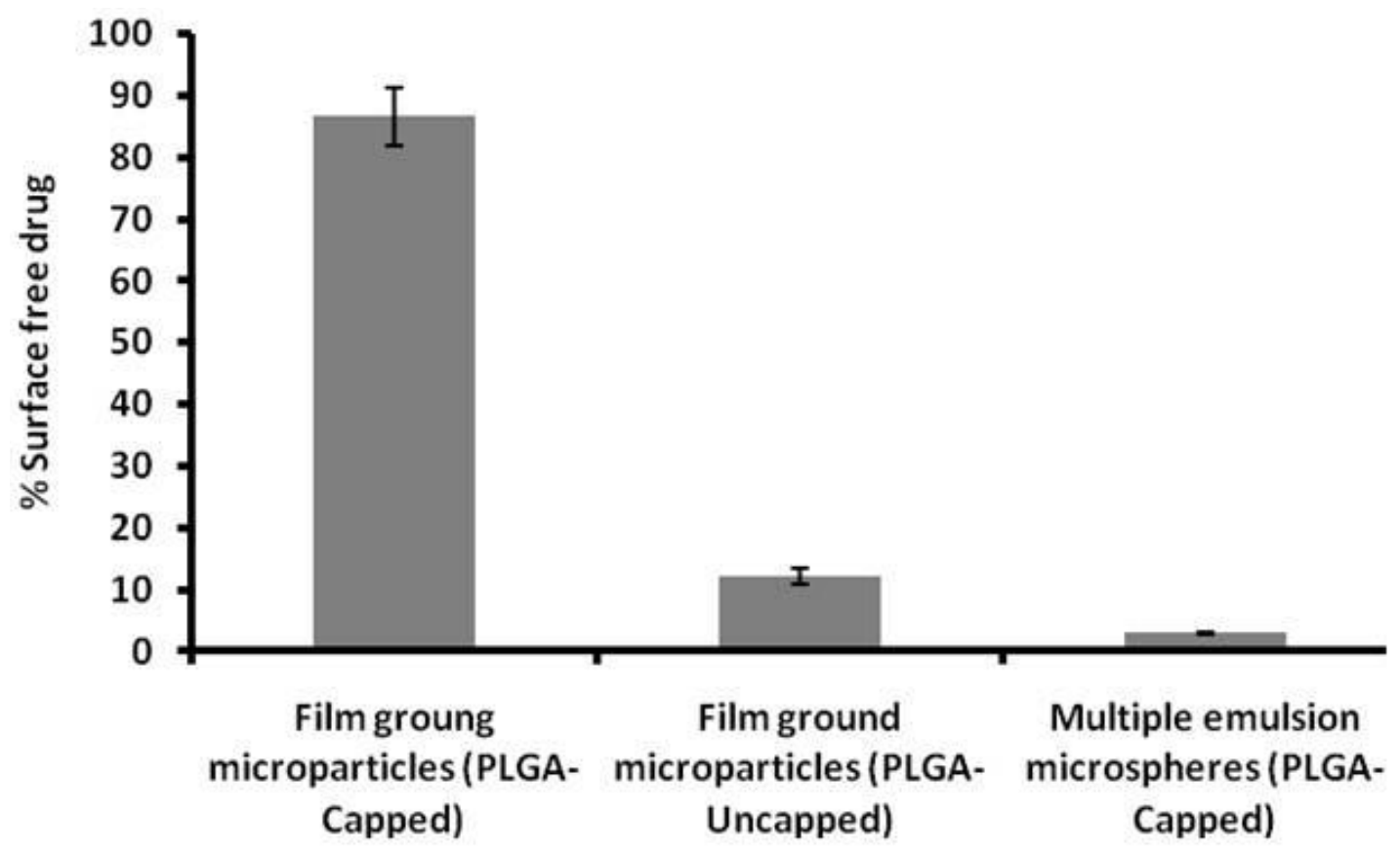

Figure 4-3. Surface free drug on PLGA micro particles/microspheres. 

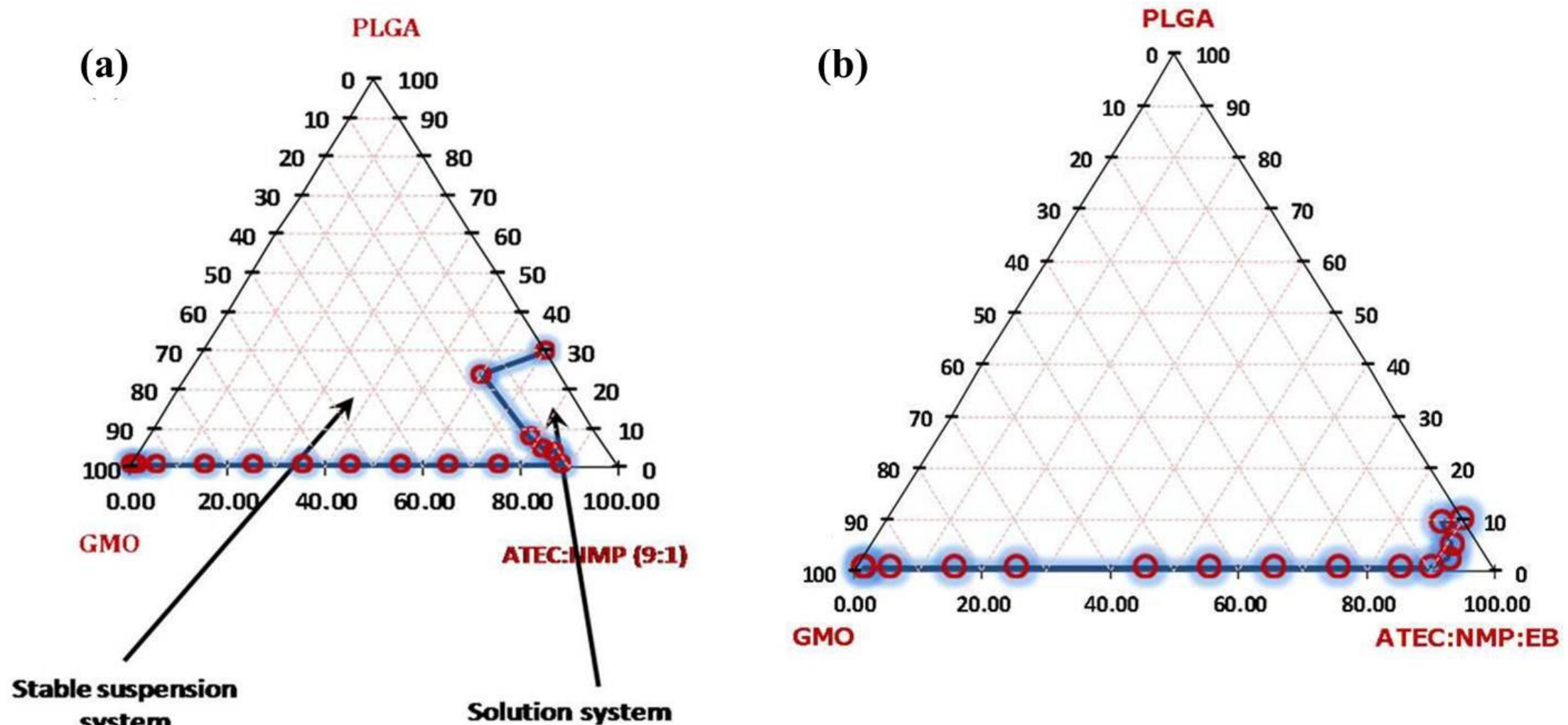

Figure 4-4. Ternary phase diagrams for the injection vehicle (a) binary solvent based system; and (b) ternary solvent based system. 


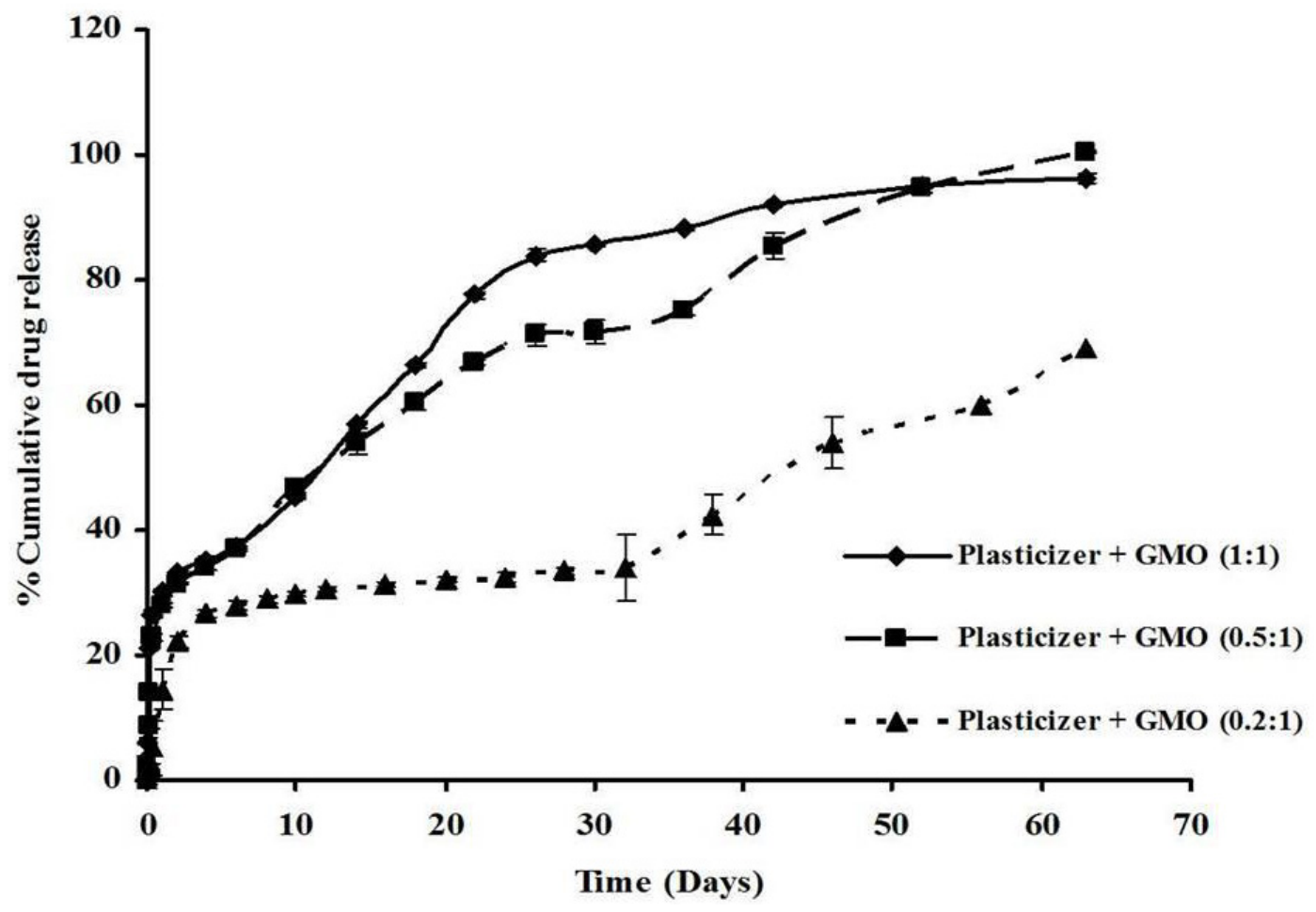

Figure 4-5. In vitro drug release from PLGA micro particles in presence of various plasticizer: GMO ratios.

Notes: Each data point corresponds to an average of three measurements and the associated standard deviation.

Plasticizer refers to the combination of NMP and ATEC in a ratio of 1:9 w/w. 


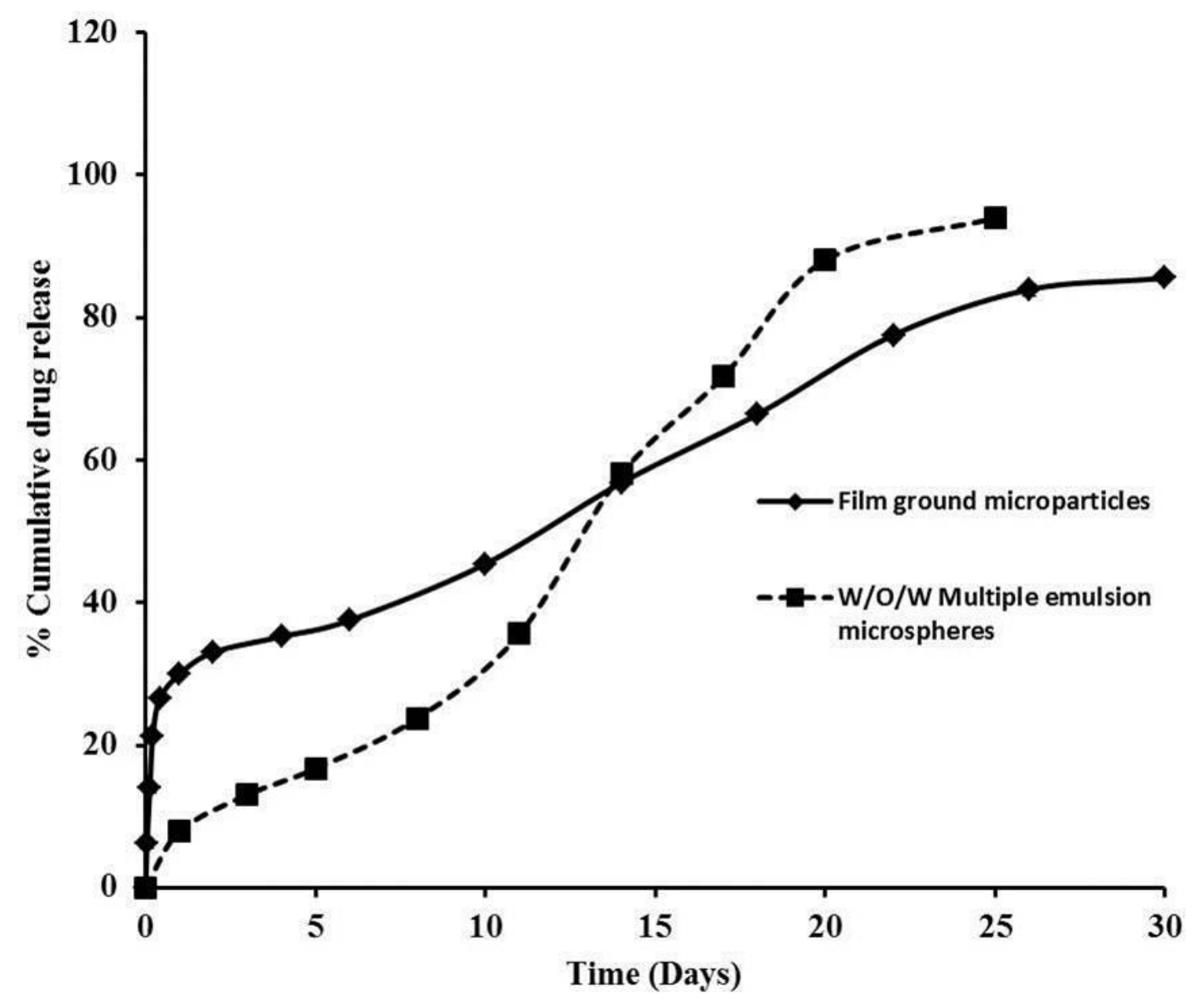

Figure 4-6. In vitro drug release from PLGA micro particles prepared by using two different methods.

Notes: Each data point corresponds to an average of three measurements and the associated standard deviation.

Plasticizer refers to the combination of NMP and ATEC in a ratio of $1: 9 \mathrm{w} / \mathrm{w}$. 
hydrophobic plasticizers surpasses the tendency of the PLGA polymer to show a plateau phase of minimal drug release and causes the drug to be released in a continuous diffusion controlled manner. Table 4-1 shows the average drug release rates from PLGA film ground micro particles obtained by using different injection vehicle compositions. The release rate increased upon increasing the proportion of the polymer plasticizer in vehicle composition. This was essentially due to the formation of a more fluid polymer matrix in presence of large amount of plasticizer(s) that resulted in polymer chain relaxation and a continuous diffusion of drug molecules into the dissolution medium.

\subsubsection{Effect of polymer end group on drug release}

The effect of the PLGA end group (acid or ester) showed a twofold effect on drug release from the in situ micro particulate formulations. First, the acid end group in the PLGA polymer accelerated the drug release during the final stage resulting in a complete drug release relatively faster than the polymer with ester end group. This can be explained easily by the known higher degradation rate of the PLGA polymer chains with acid end group. ${ }^{125}$ Secondly, a lower initial drug release was observed with the acid end group polymer, which can be attributed to an overall lower amount of surface free drug associated with the micro particles made with acid end group PLGA polymer (Figure 4-7). This is in conformation with a similar observation made earlier by Luan and Bodmeier, ${ }^{126}$ where the acid end group polymer exhibited a lower initial burst release of drug compared to the ester end group polymer. An ionic interaction between the terminal carboxylic end groups of PLGA and the two basic amino acids (arginine and histidine) of the drug leuprolide acetate as reported by Okada and co-workers further explains the observations. 114

\subsubsection{Effect of a binary vs. ternary solvent system on drug release}

Ethyl benzoate (EB) is a comparatively more hydrophobic solvent than both NMP and ATEC. The Hildebrand solubility parameter has been reported to be $23.1 \mathrm{MPa}^{1 / 2}$ for $\mathrm{NMP},{ }^{127} 19.2 \mathrm{MPa}^{1 / 2}$ for $\mathrm{ATEC}^{34}$ and $8.2 \mathrm{MPa}^{1 / 2}$ for $\mathrm{EB}^{127}$. This essentially indicates a slower phase inversion of the polymer due to a weak solvent-non solvent affinity (EB-water) for EB relative to both NMP and ATEC. EB therefore slows down the overall rate of drug release. Further the drug release is more gradual and continuous with the additional presence of EB again due to a lower water solubility of EB and therefore the existence a more fluid polymer matrix for a longer time (Figure 4-8). The initial drug release was not affected significantly due to the additional presence of EB possibly due to the presence of other plasticizers i.e. NMP and ATEC in the formulation. The ternary phase diagram for the vehicle system containing EB along with both NMP and ATEC demonstrates a relatively broader range of plasticizer and GMO concentrations that can be used to suspend or disperse the drug loaded PLGA micro particles without significant gelation/plasticization of PLGA polymer prior to administration (Figure 4-4 (b)). This can attributed to the large difference in the solubility parameters of PLGA polymer and EB reported to be $\left(22.3 \mathrm{MPa}^{1 / 2}\right)^{128}$ and $\left(8.2 \mathrm{MPa}^{1 / 2}\right)$ for PLGA and EB respectively which 
Table 4-1. Drug release rates from PLGA micro particles in presence of different ratio proportions of plasticizer: GMO.

\begin{tabular}{lc}
\hline Plasticizer: GMO (w/w) & Drug release rate $(\boldsymbol{\mu g} / \mathbf{h r})$ \\
\hline & \\
$0.2: 1$ & 2.18603 \\
$0.5: 1$ & 4.01834 \\
$1: 1$ & 4.09447 \\
\hline
\end{tabular}

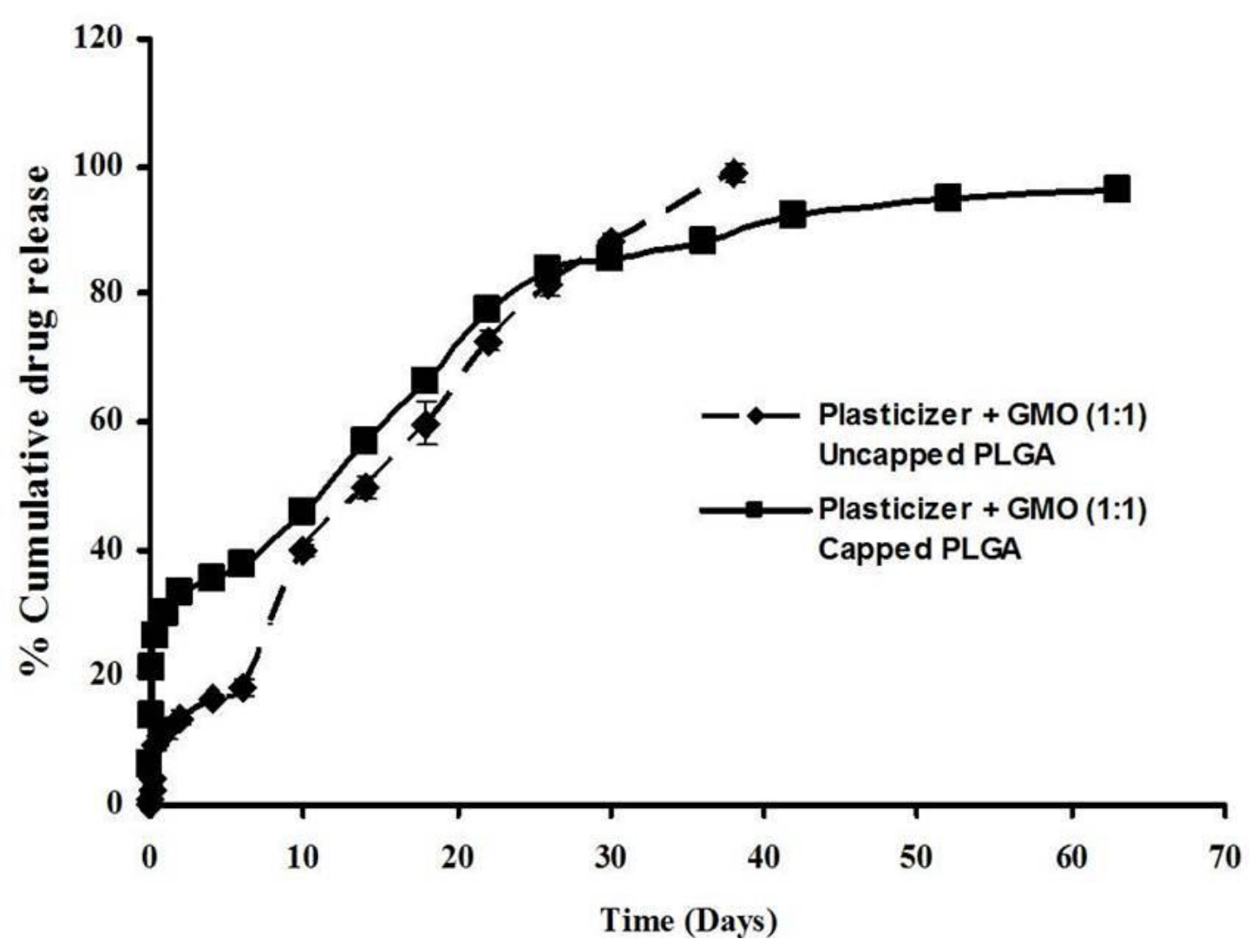

Figure 4-7. In vitro drug release from PLGA micro particles prepared by using two different end groups.

Notes: Each data point corresponds to an average of three measurements and the associated standard deviation.

Plasticizer refers to the combination of NMP and ATEC in a ratio of 1:9 w/w. 


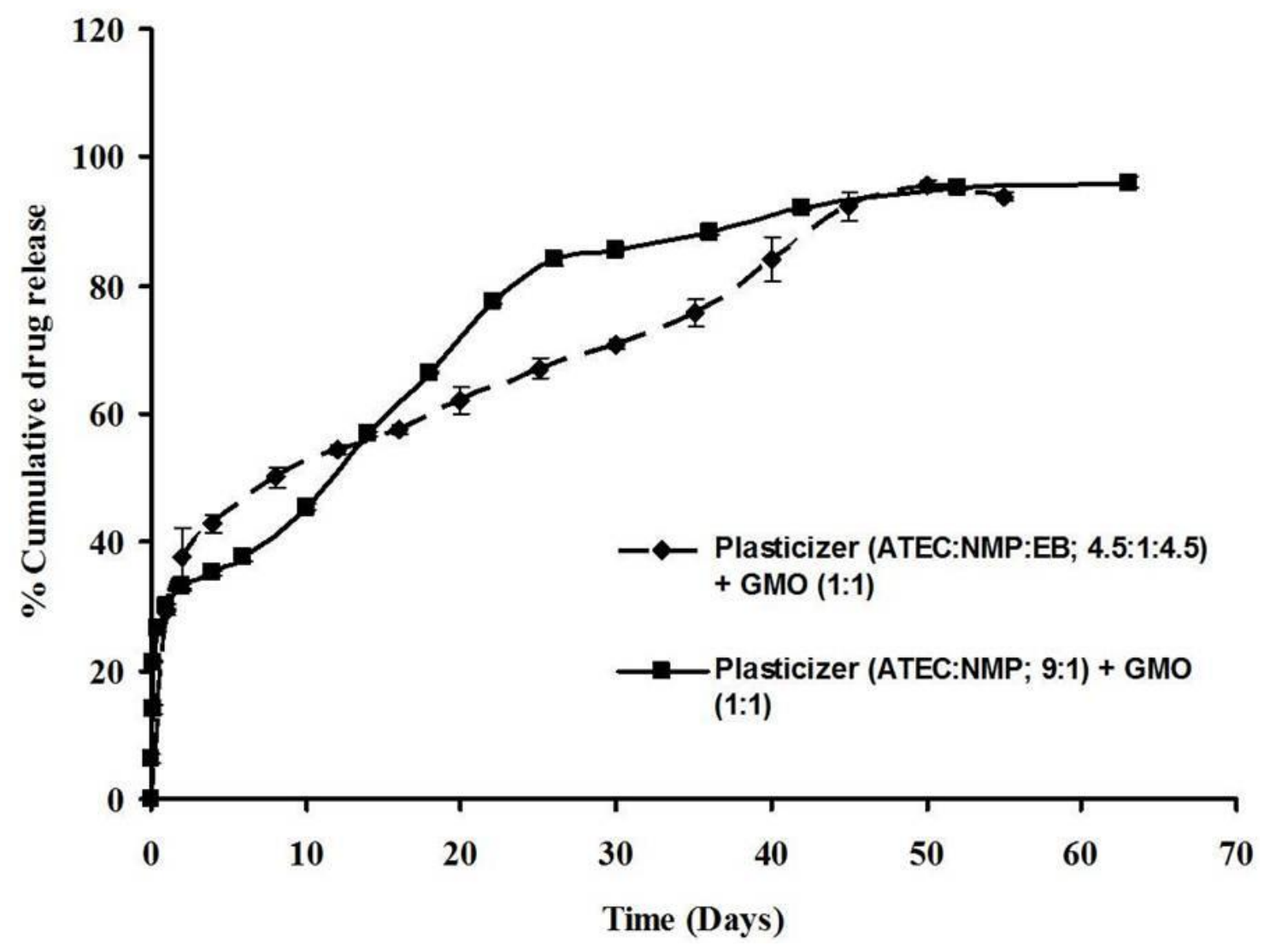

Figure 4-8. In vitro drug release from PLGA micro particles in presence and absence of the most hydrophobic solvent, EB.

Notes: Each data point corresponds to an average of three measurements and the associated standard deviation.

Plasticizer refers to the combination of NMP and ATEC in a ratio of 1:9 w/w. 
could be responsible for a delayed interaction between EB and PLGA.

\subsubsection{Correlation between drug and plasticizer release and lipid structures}

Figure 4-9 shows relationship between percent cumulative drug release and percent cumulative plasticizer release during in vitro drug release study. A lower w/w ratio of the plasticizer to GMO $(0.2: 1 \mathrm{w} / \mathrm{w})$ demonstrated a three phase relationship between the drug and plasticizer release. First and third phases exhibited a higher plasticizer release whereas there was a greater drug release compared to the plasticizer release during the second phase. This indicated essentially an erosion controlled drug release process beginning at the third phase with the first two phases confirming well with the burst phase of drug release. Upon increasing the ratio proportion of the plasticizer in vehicle composition, the relationship between drug and plasticizer became almost linear indicating a transformation into diffusion controlled drug release process. Furthermore, a relatively greater amount of drug was released compared to plasticizer released for formulations containing higher overall plasticizer content. This indicated greater relaxation of polymer chains in presence of higher plasticizer amounts and therefore a higher degree of free diffusion path for the drug molecules. The $1: 1 \mathrm{w} / \mathrm{W}$ vehicle composition essentially showed a drug release which was dependent on plasticizer diffusion and the drug release was therefore mainly controlled by the degree of polymer plasticization (Table 4-1). The second component of injection vehicle i.e. GMO being completely polymer immiscible, did not interact with the polymer. Instead, it formed lipid liquid crystalline structures that partially/completely surrounded the drug loaded PLGA micro particles and possibly created an additional barrier for drug release. Figure 4-10 shows different lipid structures formed after equilibration of formulation mass with excess of water in dissolution media. The gelled lipid structure formed from formulation with least amount of plasticizer resembled the characteristic liquid crystalline network of the glycerol lipids. The lipid structure formed at the highest concentration of plasticizer in formulation, however, resembled an aggregated network of lipid vesicles. Such a structure has been identified as a W/O micro emulsion phase (aka emulsified micro emulsion, EME). ${ }^{129}$ Yaghmur and co-workers have studied the addition of tetradecane induced transition of the internal structure of mono-olein-water system from

the bi-continuous cubic phase to hexagonal to the isotropic liquid phase (EMEs). ${ }^{130}$ The intermediate concentration of plasticizer resulted in formation of a lamellar phase in association with the emulsion phase. The differences in the microcrystalline structures of the lipid assemblies at various ratios of plasticizer to GMO were further examined and confirmed by DSC studies (Figure 4-11). All three samples showed similar endothermic events but with the events occurring at different temperatures. Furthermore, the samples showed presence of two endotherms after equilibration with excess of water. The position of the endotherms was however, different among samples indicating the disruption of lamellar structure by the emulsion system. Furthermore, lowest enthalpy values were observed at highest plasticizer to GMO ratio, indicating least amount of lamellar constitution in the particular sample.

Overall, the drug release process was more diffusion controlled and was entirely 


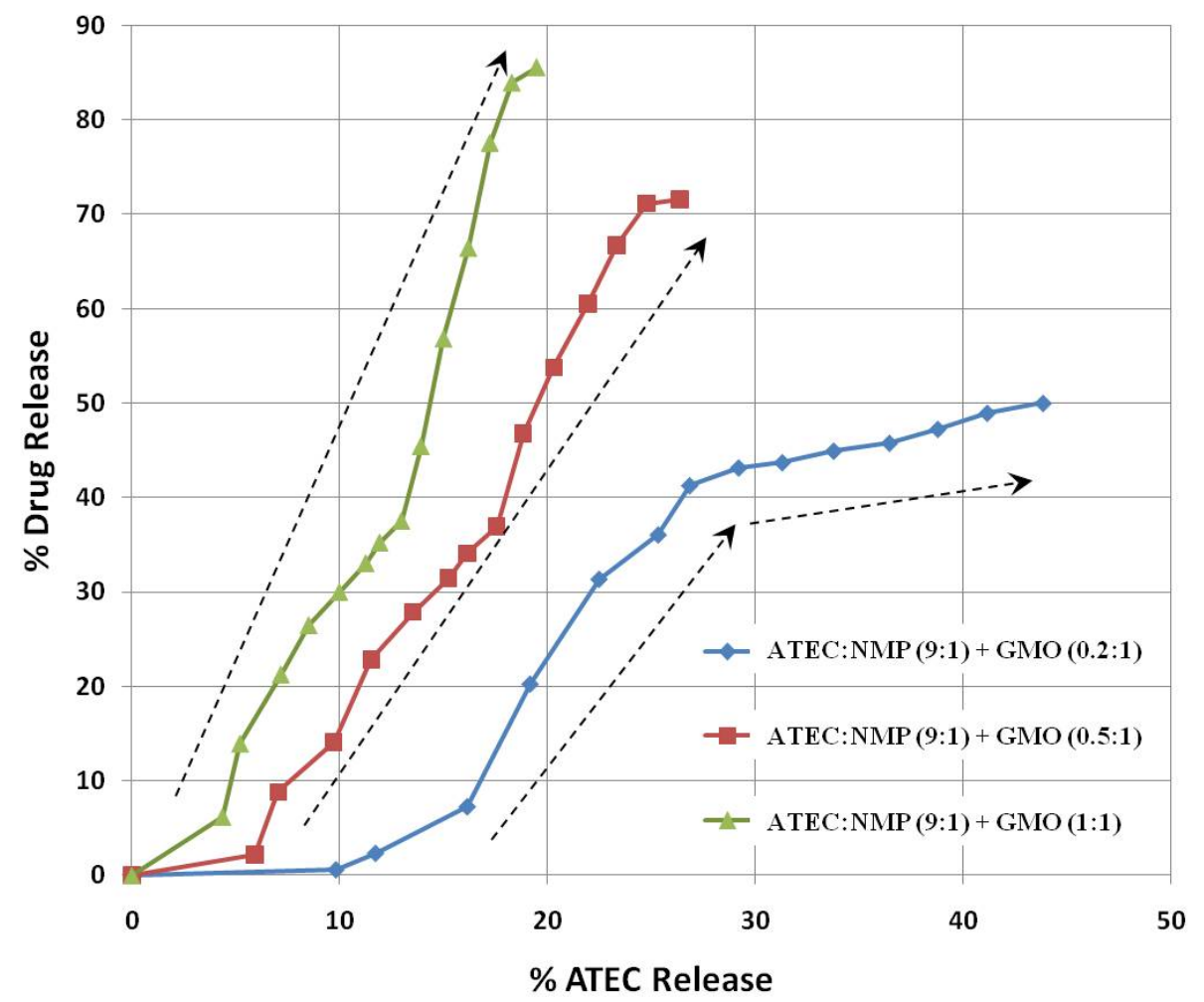

Figure 4-9. Correlation between cumulative drug release and plasticizer release from formulation depot. 

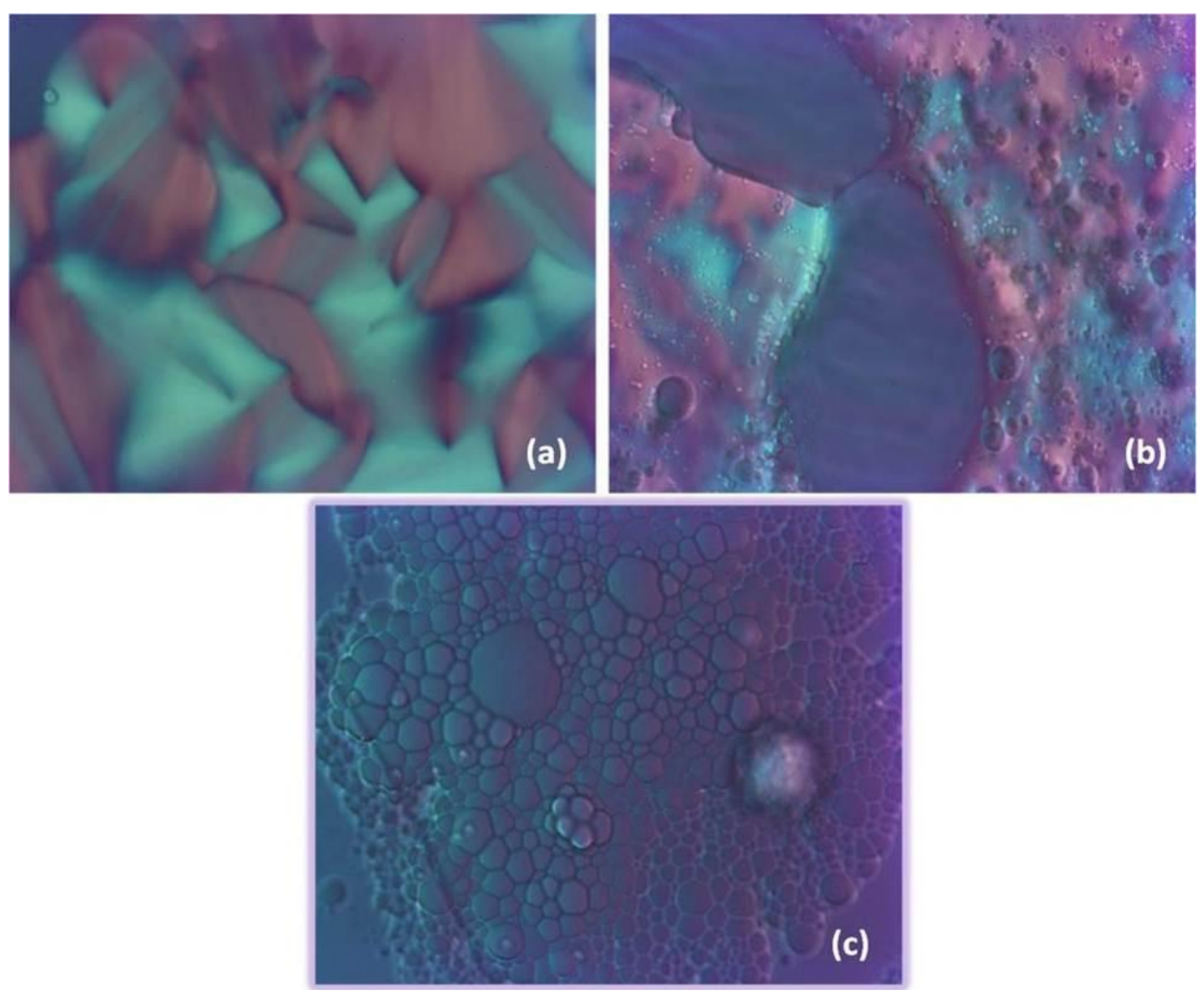

Figure 4-10. Various lipid microcrystalline structures formed after equilibration with excess of water of the formulation with following injection vehicle compositions: (a) ATEC:NMP (9:1):GMO (0.2:1); (b) ATEC:NMP (9:1):GMO (0.5:1); and (c) ATEC:NMP (9:1):GMO (1:1). 


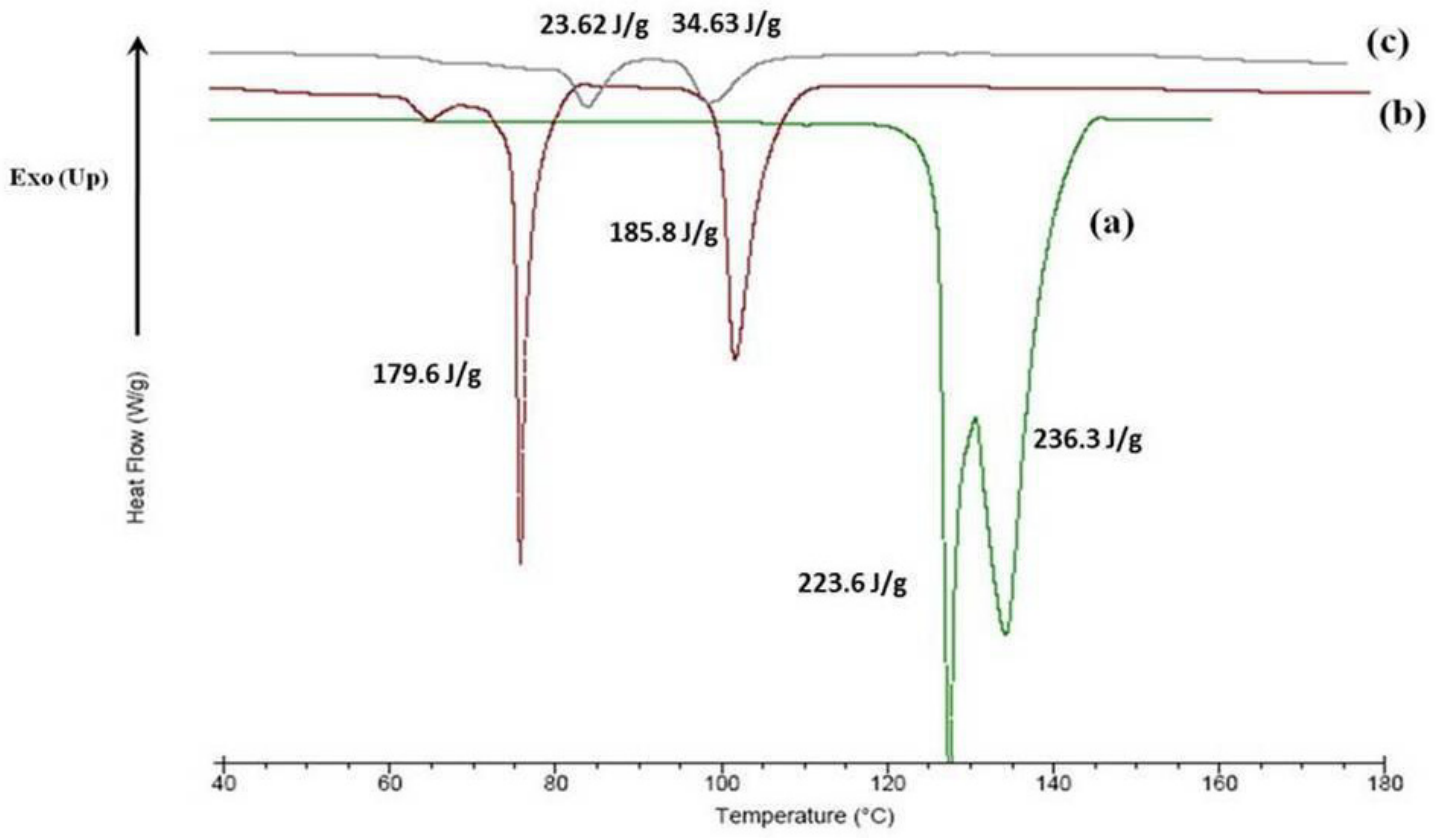

Figure 4-11. DSC thermograms of various lipidic microcrystalline structures: (a) ATEC:NMP (9:1):GMO (0.2:1); (b) ATEC:NMP (9:1):GMO (0.5:1); and (c) ATEC:NMP (9:1):GMO (1:1). 
controlled by the solvent diffusion from the formulation depot at higher ratios of plasticizer to GMO. Decreasing the total amount of plasticizer in the formulation resulted in a less dominant diffusion process and the drug release became more erosion controlled.

\subsubsection{Mathematical modeling of drug release}

The mathematical equations used to model drug release from the PLGA microparticulate formulation in novel injection vehicle are summarized in Table 4-2. Peppas-Sahlin model demonstrated the best fit for drug release rate as indicated by the $\mathrm{R}^{2}$ values (Table 4-3). Ratios for the polymer chain relaxation (R) and fickian diffusion (F) coefficients were calculated using the Peppas-Sahlin equation and results are summarized in Table 4-4. The values for the R/F ratio clearly indicate a more fickian diffusion controlled drug release process upon increasing the plasticizer to GMO ratio in the injection vehicle.

\subsubsection{Macro and micro environmental $\mathrm{pH}$}

The standard curves for measuring fluorescent intensity as a function of $\mathrm{pH}$ of dissolution media are depicted in Figure 4-12, where both fluorescein and oregon green showed a polynomial relationship between the fluorescent intensity and the $\mathrm{pH}$ of dissolution media. The dye concentrations in dissolution media were optimized in order to find the respective concentrations of both the dyes at which the change in fluorescent intensity is highly sensitive to change in $\mathrm{pH}$ of the media. Figure 4-13 shows micro environmental as well as the external $\mathrm{pH}$ in dissolution media as a function of time. Calculated p-values indicated a significant difference between the micro environmental and external $\mathrm{pH}$ values for formulation containing lower ratios of plasticizer to GMO in the injection vehicle. This essentially indicated that apart from the drug, the polymer acidic degradation products were also released by erosion dominated process. The $\mathrm{p}$-value for the formulation with highest amount of plasticizer, however, showed an insignificant difference between micro environmental and external $\mathrm{pH}$ values which explains diffusion controlled release of the acid degradation products of polymer into the dissolution media.

\subsubsection{Drug release rate and morphological changes}

Three distinct mechanisms could be identified upon plotting drug release rate vs. time (Figure 4-14). First phase ( 0 to 8 days) demonstrates a sharp increase in drug release rate attributed to the surface adsorbed drug on micro particles immediately followed by a steep decline in the release rate. This decline could be attributed to a high permeability of the formulation depot resulting from an increase in pore-density on the depot surface. This was followed by a second slow increase in the release rate indicating a higher polymer chain rearrangement and more drug molecules exposed to the aqueous channels in the depot ( 8 to 20 days). This phase is known as the medium permeability 
Table 4-2. Mathematical equations for modeling drug release.

\begin{tabular}{lc}
\hline Model & Equation \\
\hline & \\
${ }^{\mathrm{a}}$ Zero order & $\mathrm{F}=\mathrm{k}_{0} * \mathrm{t}$ \\
${ }^{\mathrm{b}}$ First order & $\mathrm{F}=100 *\left[1-\mathrm{Exp}\left(-\mathrm{k}_{1} * \mathrm{t}\right)\right]$ \\
${ }^{\mathrm{c}}$ Higuchi & $\mathrm{F}=\mathrm{k}_{\mathrm{H}} * \mathrm{t}^{\wedge} 0.5$ \\
${ }^{\mathrm{d}}$ Korsenmeyer Peppas & $\mathrm{F}=\mathrm{k}_{\mathrm{KP}} * \mathrm{t}^{\wedge} \mathrm{n}$ \\
${ }^{\mathrm{e}}$ Peppas Sahlin & $\mathrm{F}=\mathrm{k}_{1} * \mathrm{t}^{\wedge} \mathrm{m}+\mathrm{k}_{2} * \mathrm{t}^{\wedge}(2 * \mathrm{~m})$ \\
${ }^{\mathrm{f}}$ Kopcha & $\mathrm{F}=\mathrm{k}_{1} * \mathrm{t}^{\wedge} 0.5+\mathrm{k}_{2} * \mathrm{t}$ \\
\hline
\end{tabular}

Notes: ${ }^{\mathrm{a}} \mathrm{k}_{0}$ is the zero order release rate constant.

${ }^{b} k_{1}$ is the first order release rate constant.

${ }^{c} \mathrm{k}_{\mathrm{H}}$ is the Higuchi release constant.

${ }^{d} k_{K P}$ is the release constant incorporating structural and geometric characteristics of the drug-dosage form; $\mathrm{n}$ is the diffusional exponent indicating the drug-release mechanism. ${ }^{e} k_{1}$ is the constant related to the fickian kinetics; $k_{2}$ is the constant related to Case-II relaxation kinetics; $\mathrm{m}$ is the diffusional exponent for a device of any geometric shape which inhibits controlled release.

${ }^{\mathrm{f}} \mathrm{k}_{1}$ is the constant denoting the relative contribution of $\mathrm{t}^{0.5}$-dependent drug diffusion to drug release; $\mathrm{k}_{2}$ is the constant denoting the relative contribution of $\mathrm{t}$-dependent polymer relaxation to drug release. 
Table 4-3. Calculated $\mathrm{R}^{2}$ values from various mathematical models.

\begin{tabular}{|c|c|c|c|c|c|c|}
\hline \multirow[t]{2}{*}{ Formulation } & \multicolumn{6}{|c|}{$\mathbf{R}^{2}$ value } \\
\hline & Zero order & First order & Higuchi & $\begin{array}{c}\text { Korsenmeyer } \\
\text { Peppas } \\
\end{array}$ & Peppas Sahlin & Kopcha \\
\hline $\begin{array}{l}\text { ATEC:NMP } \\
(9: 1)+\text { GMO } \\
(0.2: 1)\end{array}$ & 0.9248 & 0.8667 & 0.6663 & 0.9414 & 0.9525 & 0.9206 \\
\hline $\begin{array}{l}\text { ATEC:NMP } \\
(9: 1)+\text { GMO } \\
(0.5: 1)\end{array}$ & 0.9491 & 0.9843 & 0.8773 & 0.9765 & 0.9921 & 0.9703 \\
\hline $\begin{array}{l}\text { ATEC:NMP } \\
(9: 1)+\text { GMO } \\
(1: 1)\end{array}$ & 0.9831 & 0.9355 & 0.8478 & 0.8964 & 0.9506 & 0.8811 \\
\hline
\end{tabular}

Table 4-4. Comparison of relaxation vs. fickian diffusion for various injection vehicles.

\begin{tabular}{lc}
\hline Formulation injection vehicle & R/F \\
\hline ATEC:NMP (9:1) + GMO (0.2:1) & 0.3027449 \\
ATEC:NMP (9:1) + GMO (0.5:1) & 0.287617 \\
ATEC:NMP (9:1) + GMO (1:1) & 0.242275 \\
\hline
\end{tabular}




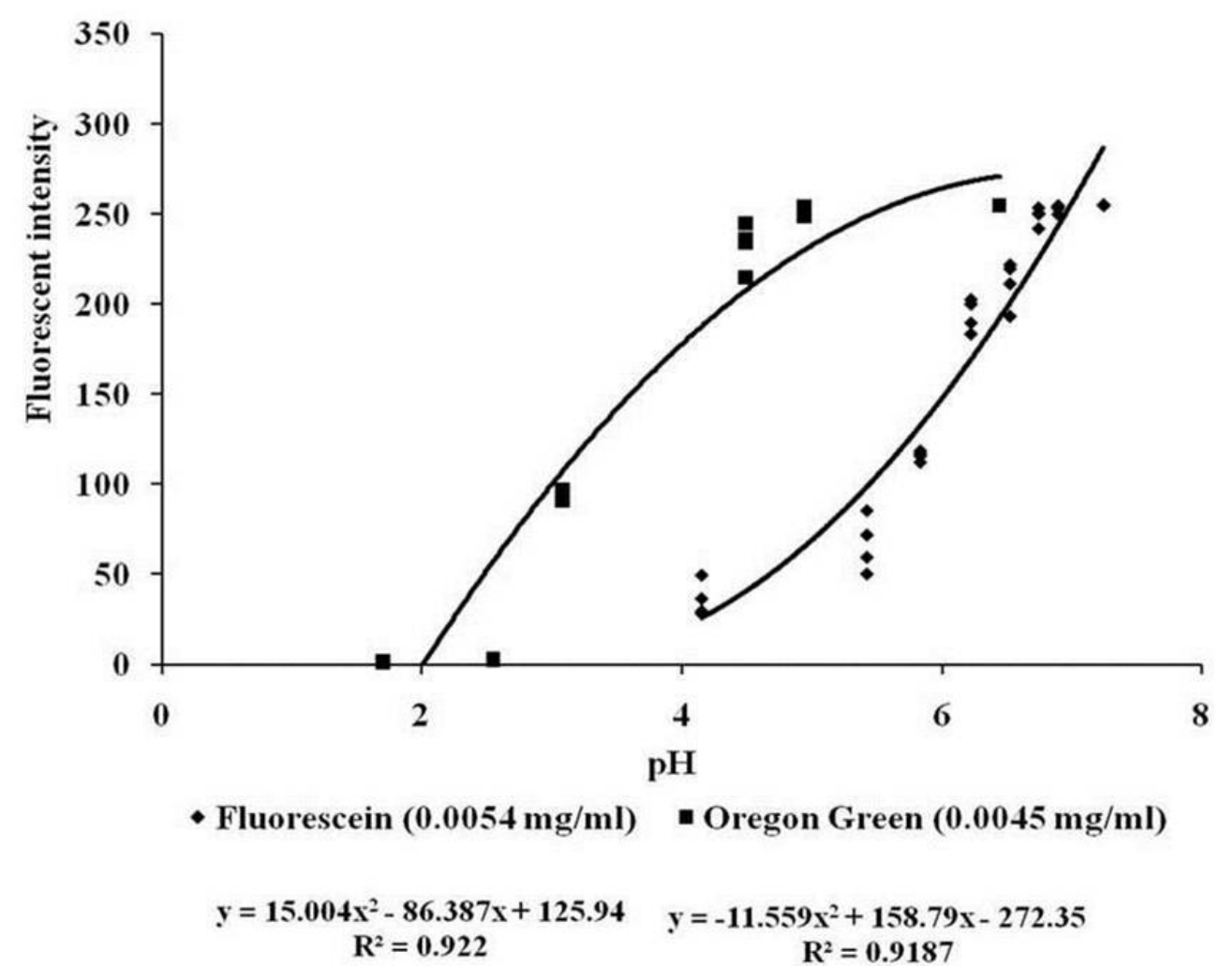

Figure 4-12. Standard curves of fluorescent dyes for confocal microscopy study. 


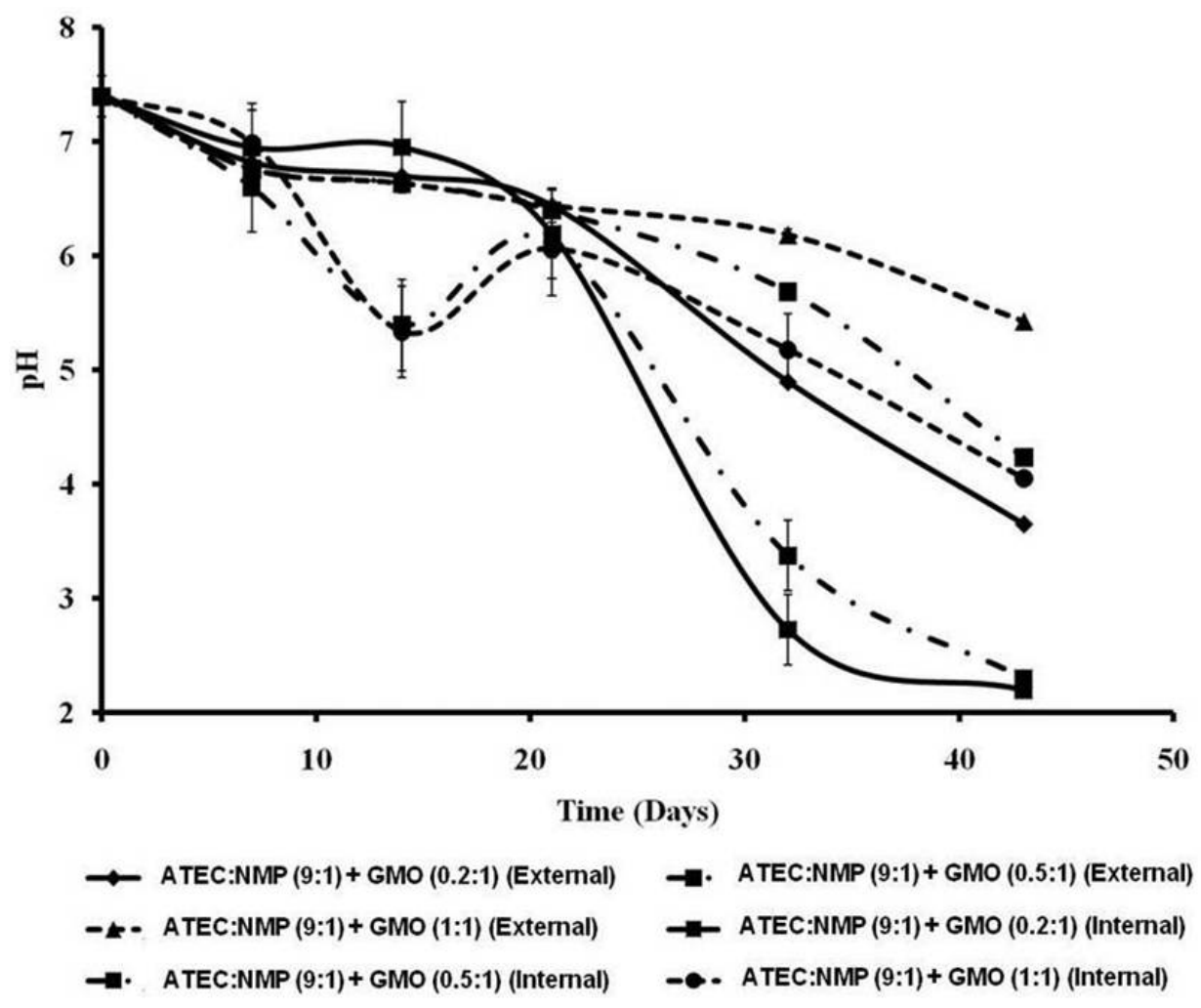

Figure 4-13. Correlation between micro environmental and external $\mathrm{pH}$ as determined by confocal microscopy. 


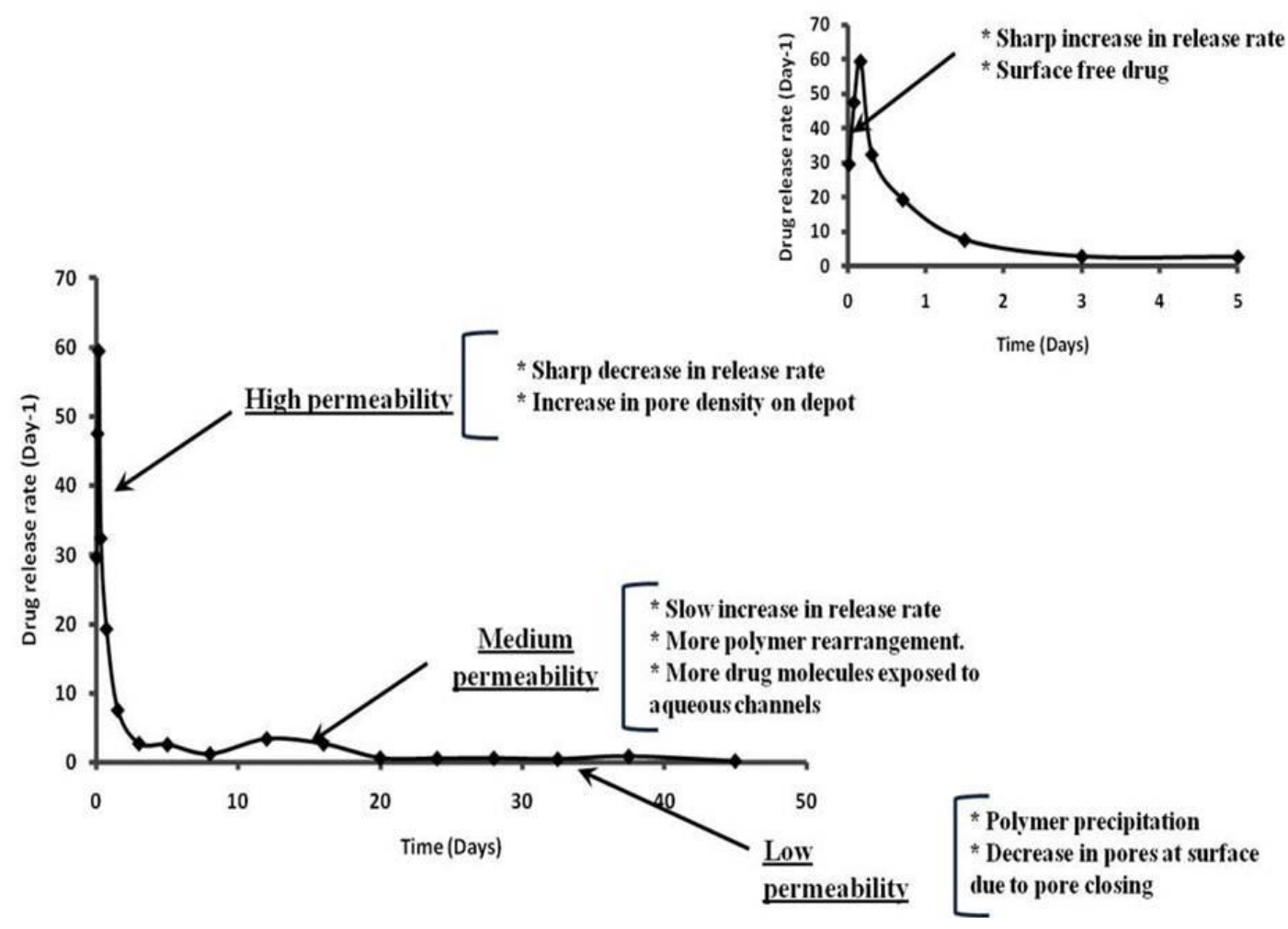

Figure 4-14. Drug release rate and morphological changes in formulation depot. 
phase and was further confirmed by light microscopy studies (Figure 4-15) that shows the presence of relatively smaller pores in the medium permeability depot structure than a high permeability structure. The final phase was a low permeability phase (20 to 40 days) where polymer precipitation took place with a simultaneous reduction in the pore-density at the depot surface caused due to pore closing.

\subsubsection{Viscosity and injection force}

The viscosity of the proposed formulation was compared with one of the marketed PLGA formulations i.e. Eligard ${ }^{\circledR}$ containing leuprolide acetate and the results are depicted in Figure 4-16. Both the injection vehicle as well the final formulation with the PLGA micro particles $(7.0 \% \mathrm{w} / \mathrm{w}$ of total injection vehicle) suspended in the injection vehicle demonstrated a significantly lower viscosity than the currently marketed formulation. This not only would provide the ease of administration but also minimize pain upon injection. Injection force was correlated with viscosity for the PLGA solutions in the plasticizer combination of ATEC:NMP $(9: 1 \mathrm{w} / \mathrm{w})$ at three different polymer loadings of $7 \%, 14 \%$ and $28 \% \mathrm{w} / \mathrm{w}$ (Figure 4-17). Results indicated a significant increase in viscosity as well as injection force on increasing the PLGA polymer concentration in the plasticizer. The injection force of the PLGA micro particle suspension, however, did not show any significant increase upon increasing the total load of polymer particles in the plasticizer (Figure 4-18). This demonstrated a much lower injection force of the polymer suspension system compared to the polymer solution system which could be promising in terms of reduced pain during administration. Further, viscosities and injections forces were compared for the injection vehicle in the presence and absence of glycerol-lipid (Figure 4-19), whereby injection forces were monitored over time. Results showed no significant difference in viscosities of the injection vehicle in presence and absence of the glycerol lipid; however, lower injection forces could be maintained for a relatively longer period of time in the presence of glycerol lipid. This confirms the polymer immiscible functionality of the lipid that would contribute not only towards ease of administration but also a more prolonged drug release.

\subsection{Conclusion}

The present work demonstrated promising features of the novel injection vehicle for PLGA micro particulate based drug delivery. Such a system not only allowed for a readily controlled drug delivery but further provided the opportunity to modulate the release mechanism from a more erosion based drug release system to essentially a diffusion based system. Drug release mechanism was confirmed by in vitro drug release studies and additionally by microscopic studies and mathematical modeling. Injection vehicle composed of hydrophobic polymer plasticizer(s) demonstrated great potential for easy to modulate controlled drug delivery. Further presence of a glycero-lipid imparted structural stability to the formulation required for easy administration by acting as a polymer immiscible component. 

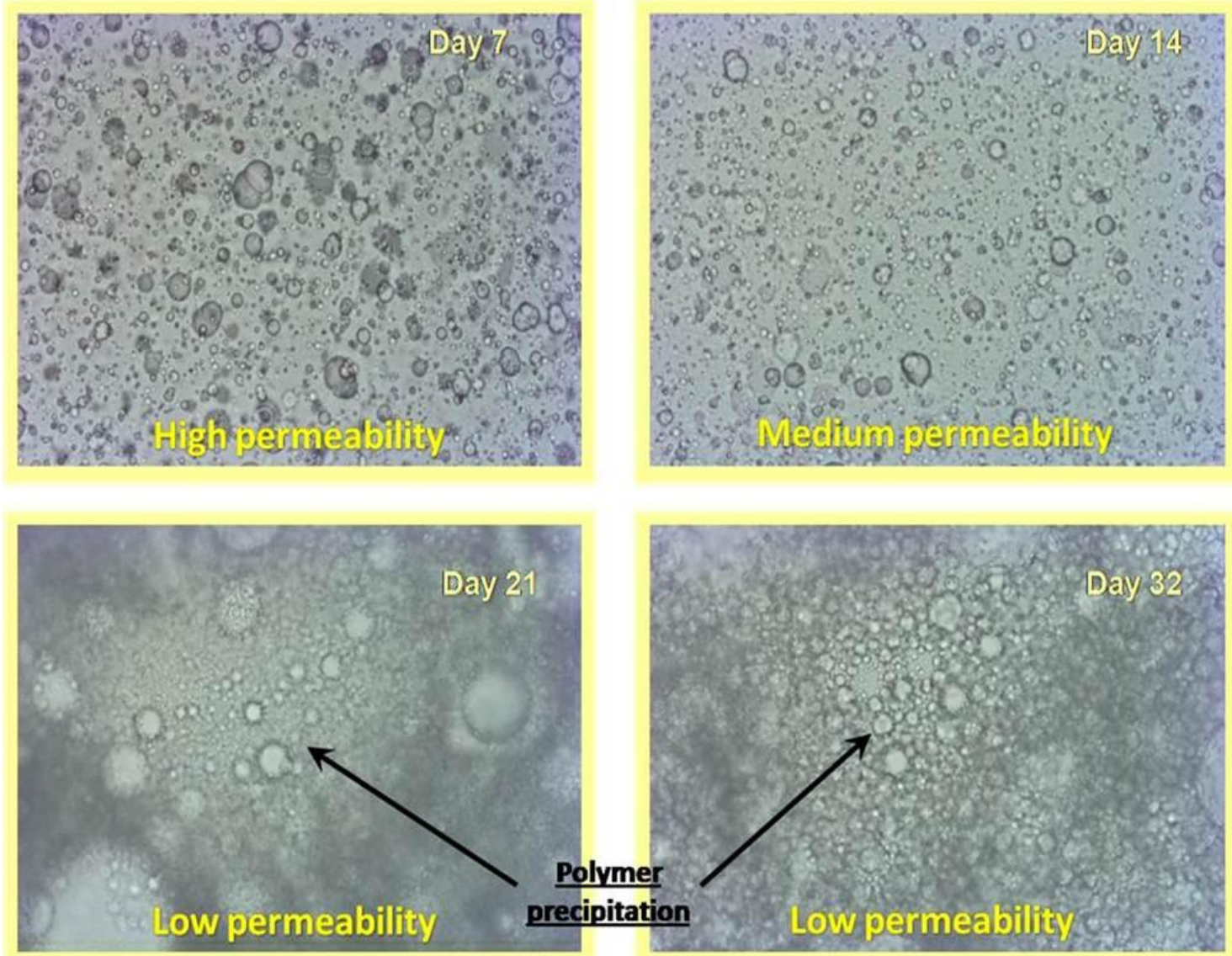

$200 \mu \mathrm{m}$

Figure 4-15. Structural changes in formulation depot during drug release. 


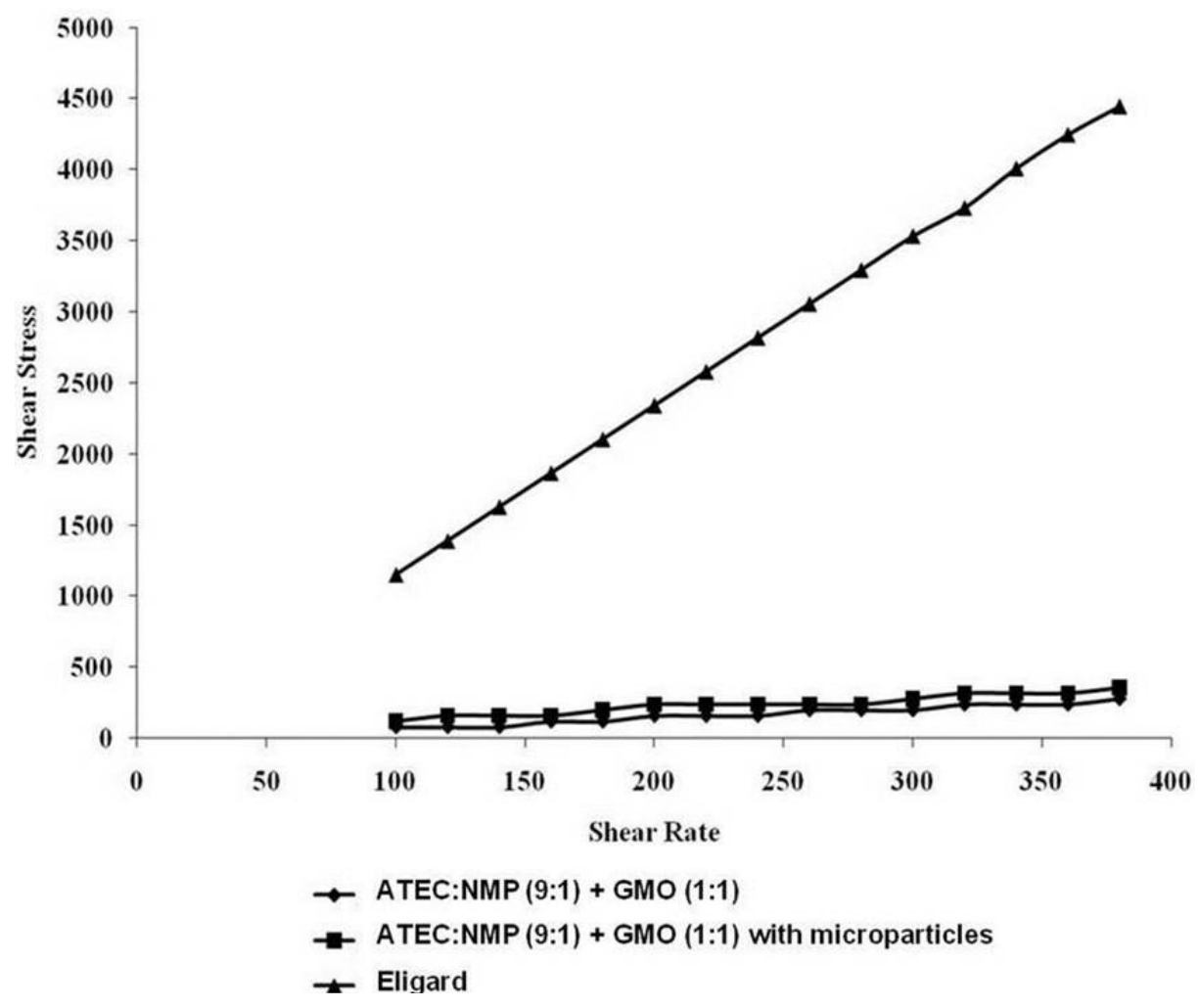

Figure 4-16. Viscosity studies of the PLGA micro particulate formulation in novel injection vehicle system. 


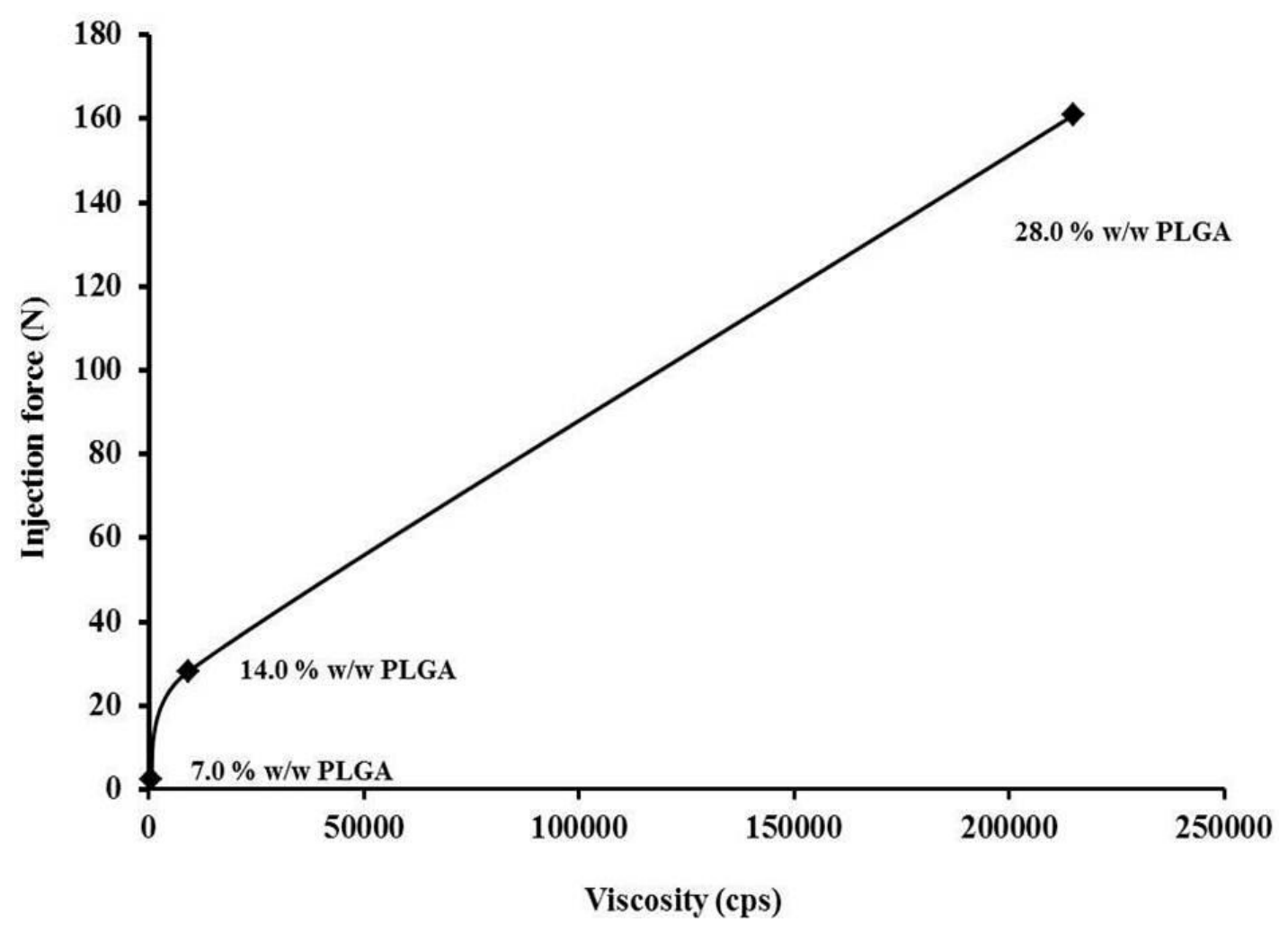

Figure 4-17. Correlation between viscosity and injection force for PLGA solution in the plasticizer.

Note: Plasticizer was selected as the combination of ATEC and NMP in a 9:1 w/w ratio. 


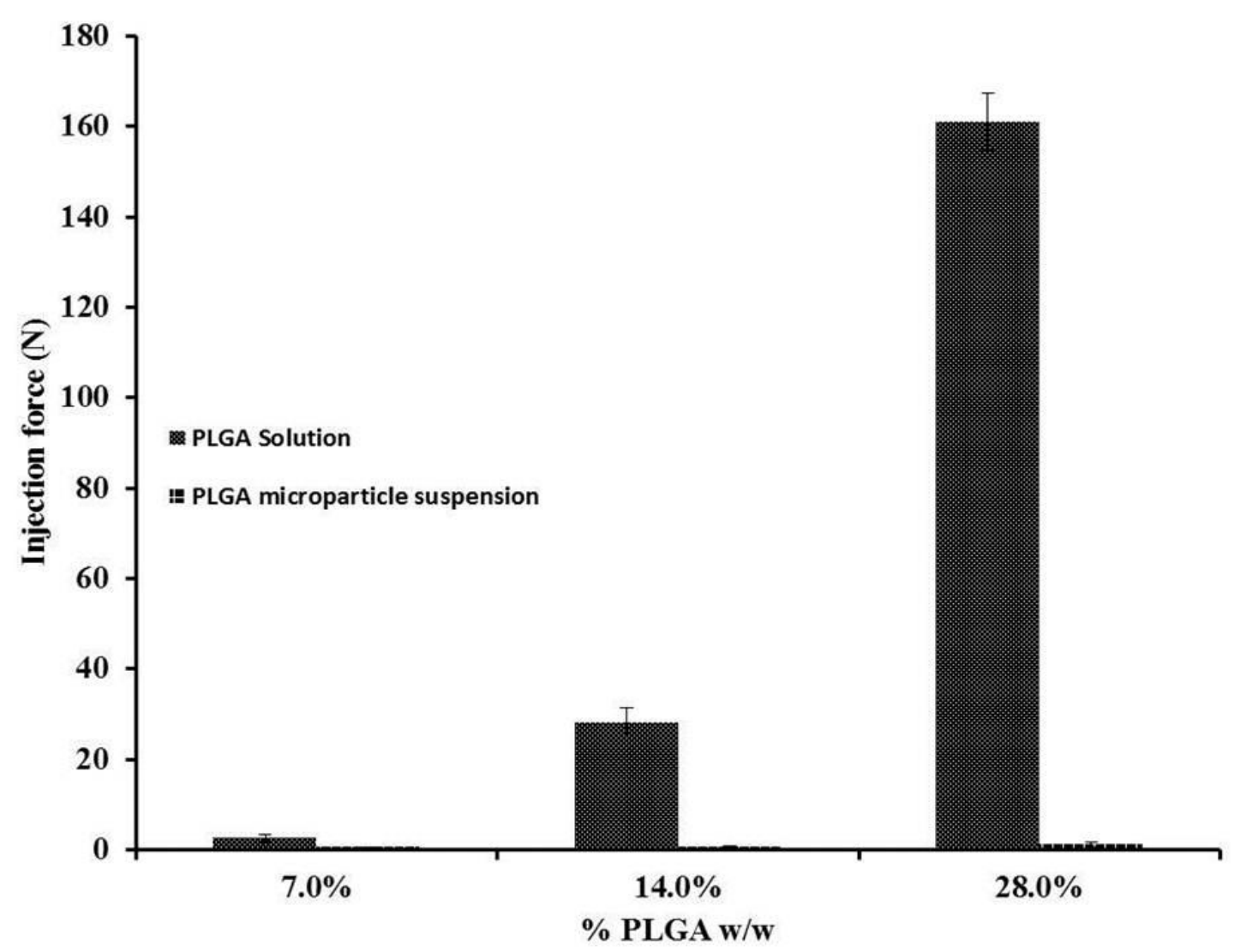

Figure 4-18. Comparison of injection force between PLGA solution and PLGA micro particle suspension in the plasticizer.

Note: Plasticizer was selected as the combination of ATEC and NMP in a 9:1 w/w ratio. 

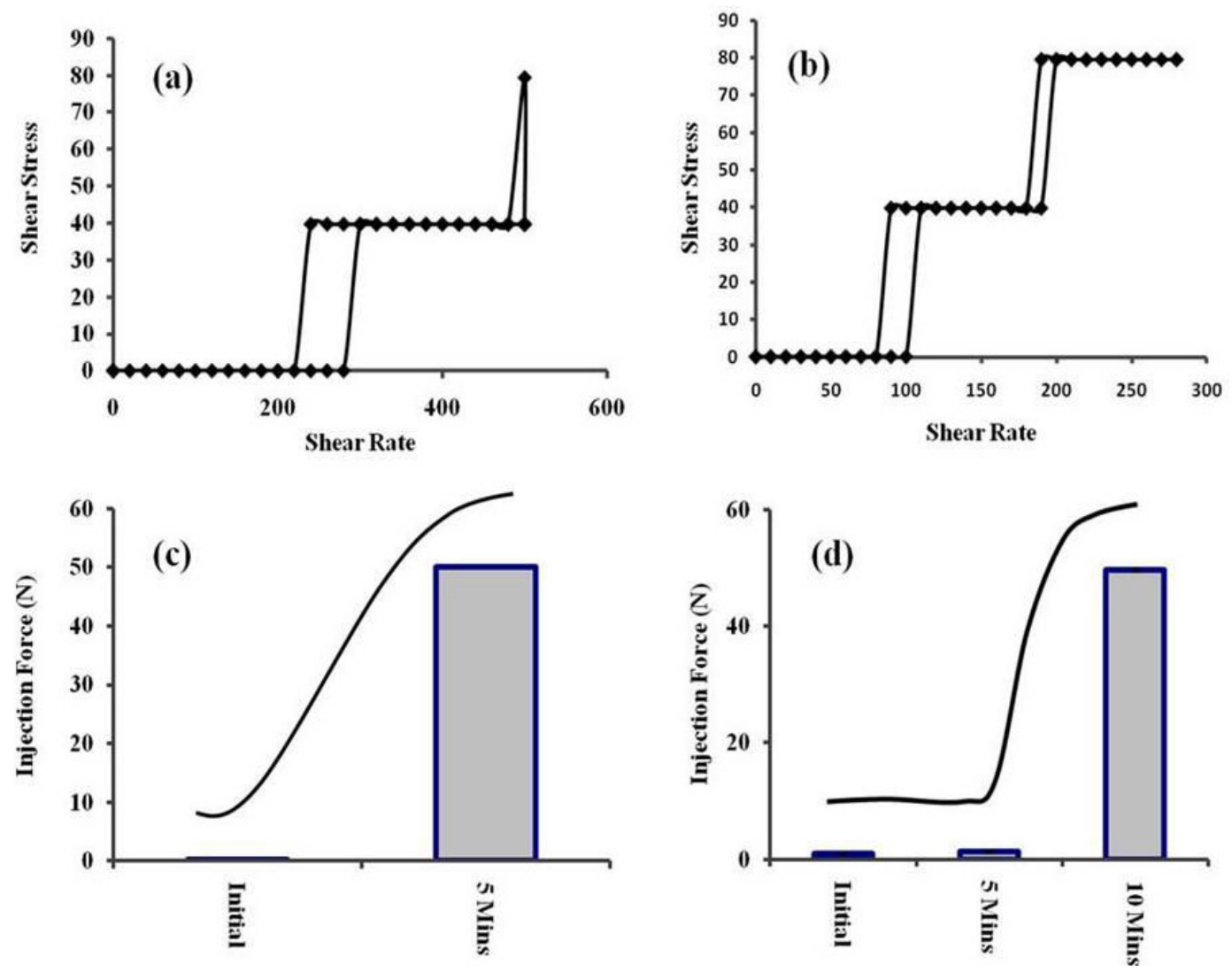

Figure 4-19. Viscosity of injection vehicle with (a) plasticizer; and (b) plasticizer and glycerol lipid and injection force of injection vehicle with (c) plasticizer; and (d) plasticizer and glycerol lipid. 


\section{CHAPTER 5. DEVELOPMENT AND CHARACTERIZATION OF PLGA MICRO PARTICLE: POLYCAPROLACTONE CRYSTALLITE BASED IN SITU IMPLANT}

\subsection{Introduction}

Polyesters form an important class of biodegradable polymers that have excellent biocompatibility and degrade via hydrolysis under human body conditions. ${ }^{131-135}$ Examples of commonly used polyesters include polycaprolactone (PCL) and poly lactic acid (PLA) polymers of which PCL exhibits the lowest degradation rate and is therefore employed in long term drug delivery. PLA on the other hand, degrades faster than PCL due to greater hydrolysis rate of its ester linkages. PCL is a low melting polymer with the melting point around $60^{\circ} \mathrm{C}$ and degrades by hydrolysis of its ester linkages in physiological conditions. Due to its excellent biodegradability and biocompatibility, it is of particular interest for the preparation of long term implants and other devices.

Degradation of PCL is an autocatalytic ${ }^{136}$ and a bulk process that can be divided into two stages:

- $\mathrm{M}_{\mathrm{N}}$ loss up to 5000 due to chain scission.

- Onset of weight loss.

Numerous different types of drug molecules such as the well-known anti-hypertensive agents, ${ }^{137}$ taxol, ${ }^{138}$ gentamicin, ${ }^{139}$ colchicine, ${ }^{140}$ chlorpromazine, ${ }^{141}$ cyclosporine, ${ }^{142}$ and cisplatin ${ }^{143}$ etc. have been encapsulated by using the PCL polymer for both controlled drug release and targeted drug delivery purposes. It's a food and drug administration (FDA) approved material for use in the human body as for example a drug delivery device or suture (sold under the brand name Monocryl). PCL has also been used together with PLGA polymers to modulate drug release characteristics. ${ }^{72}$ Apart from being used widely as carrier materials for microsphere preparation, PCL has another unique property of forming crystallites or spherulites, which is attributed to its semicrystalline nature. Such spherulites have been well studied by techniques such as scanning electron microscopy (SEM), atomic force microscopy (AFM) etc. ${ }^{144}$ Spherulites are generated when a polymer crystallizes from the melt without any disturbance. ${ }^{145,146}$ The dimensions of spherulites range from micrometers to millimeters depending on crystallizing conditions such as temperature, cooling rate and content of crystallizing agent. The individual spherulite morphology is depicted in Figure 5-1.

The crystallization process can be divided into two important stages:

5.1.1. Homogeneous nucleation: birth of primary nuclei

According to the thermodynamic theory, an embryo or nuclei has to form for the crystallization to take place. As the embryo increases in size, its free energy increases 


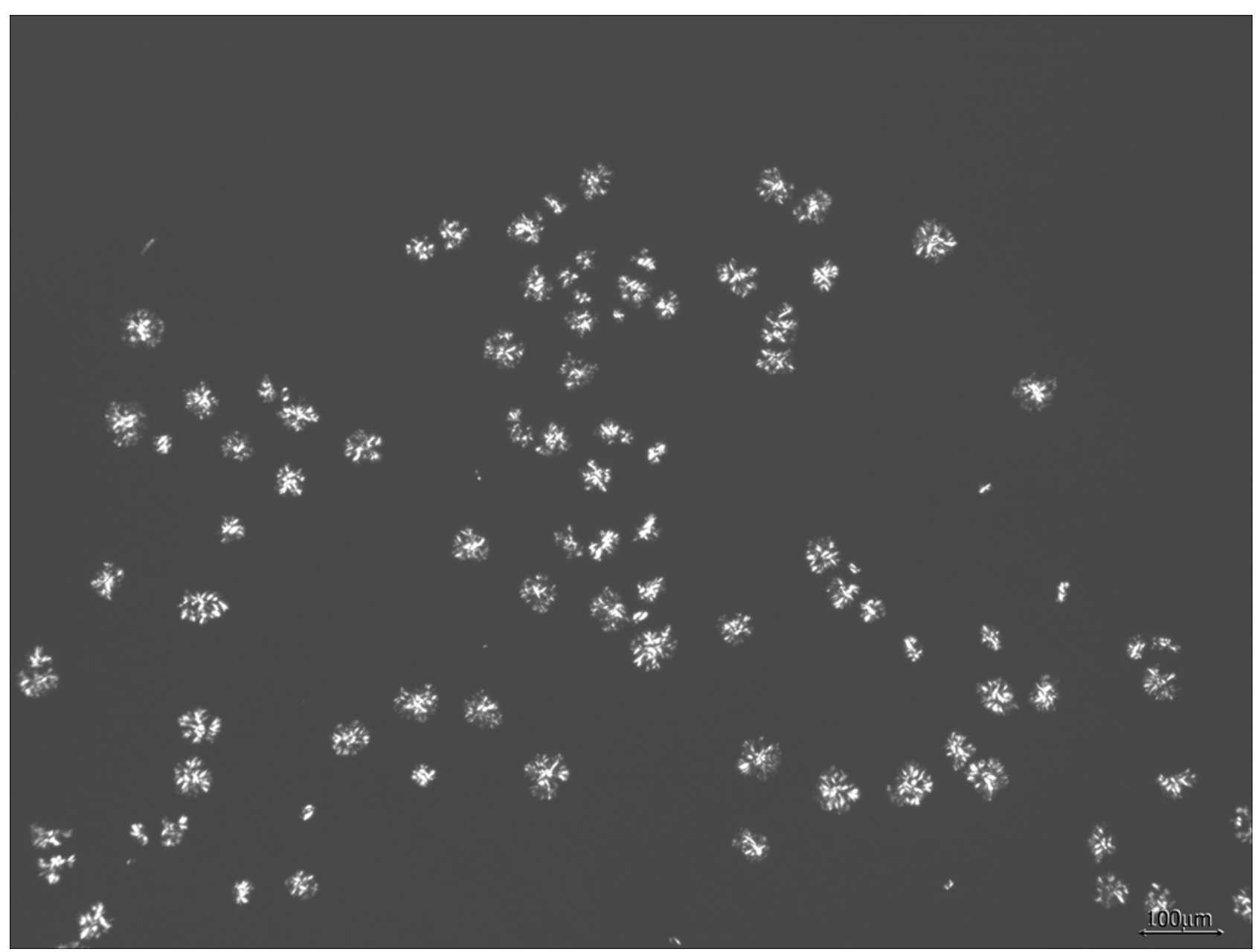

Figure 5-1. Schematic representation of the hypothetical growth of polymer spherulites. 
until it reaches a critical size beyond which the volume to surface ratio becomes large enough such that the decrease in enthalpy is larger than the increase in surface energy. The embryo is very unstable and can easily disappear below the critical size whereas above this size, it is stable. ${ }^{144}$ Figure 5-2 shows the relationship between Gibbs free energy change and nuclei growth.

\subsubsection{Development of lamellar sheaf: branching of lamellae}

Once the primary nuclei are formed, they branch and split to form a lamellar sheaf known as hedrite. A number of such lamellae are connected centrally to result in formation of a quite complex structure. For example, Beeksman et al. showed that hedrites of PCL with a high degree of complexity could be developed from the melt upon lowering the crystallization temperature to approximately $56^{\circ} \mathrm{C} .{ }^{147}$ Polymer spherulites have been used in drug delivery applications. In most cases, the drug is entrapped within the polymer matrix during the crystallization process. The effect is mutual, i.e. the incorporated drug in polymer spherulites affects the crystallite or spherulite geometry and the drug release is affected by its distribution within the polymer matrix. Incorporation of methyl red in crystalline poly hydroxyalkanoates (PHAs) has been well studied by Akhtar et al. ${ }^{148}$

This study was focused on two aspects of PCL crystallites or spherulites in drug delivery, reduction in burst release of drug from in situ implants thereby transforming the tri-phasic release pattern of drug and a high structural stability of the micro particulate suspension formulation prior to administration e.g. by providing an appropriate shear thinning and thixotropic nature to the formulation for ease of administration. The hypothesis was therefore, that the crystallites or spherulites surround the drug containing PLGA micro particles, thereby forming an additional barrier to control burst drug release from the system. Additionally, due to their stable and unique morphology, the crystallites or spherulites exhibit shear thinning and thixotropic properties that makes the micro particulate suspension system structurally stable.

\subsection{Materials and Methods}

\subsubsection{Materials}

PLGA 50:50 (IV 0.59dL/g) was obtained from Lactel absorbable polymers, Durect Corporation, Pelham, AL, USA. Glyceryl monooleate (Capmul GMO-50, EP/NF) was obtained as a generous gift from Abitec Corp., Janesville, WI, USA. NMP (N-methyl pyrrolidone; Pharmasolve) was procured from ISP Pharma Technologies, Wayne, NJ, USA, and ATEC (acetyl triethyl citrate) was obtained from Morflex Inc., Greensboro, NC, USA. Polycaprolactone (PCL) (MW 10,000) was obtained from The Dow Chemical Co., Midland, MI, USA. Leuprolide acetate was used as the model drug and was purchased from Teva Pharmaceuticals, Israel. All buffers were prepared in reverse 


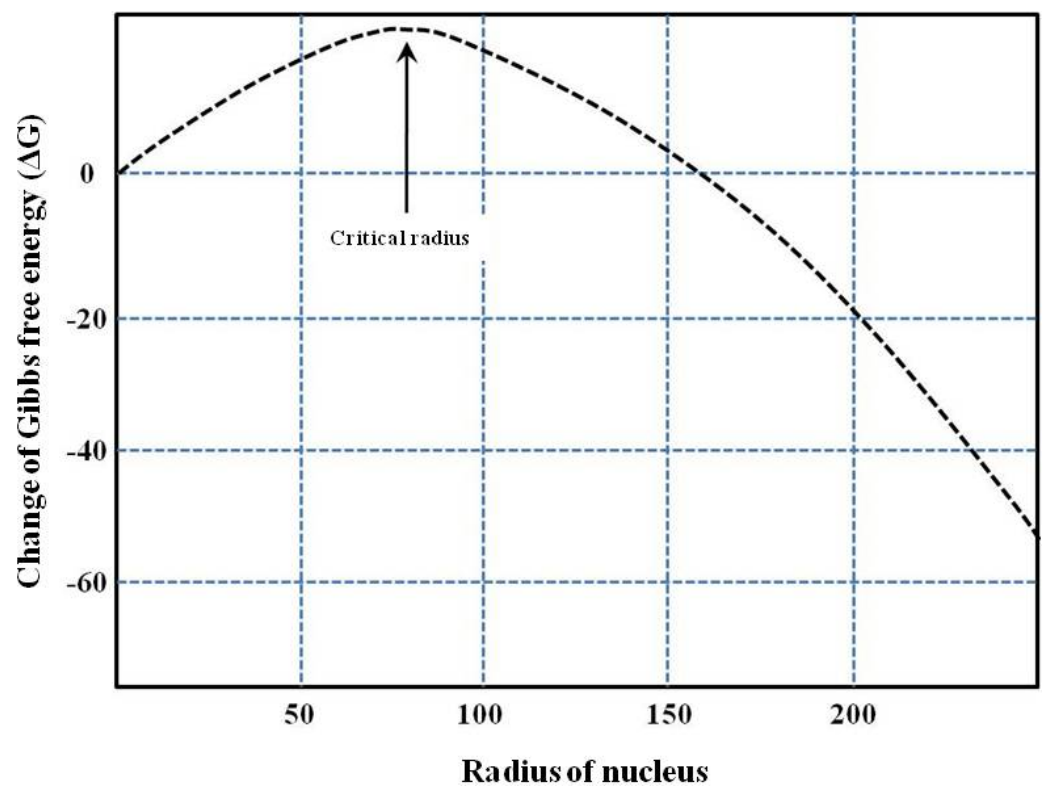

Figure 5-2. Gibbs free energy of formation of nucleus as a function of its size. 
osmosis water. Other chemicals and reagents were purchased from Sigma Aldrich.

\subsubsection{Methods}

\subsubsection{PLGA micro particle preparation}

PLGA micro particles/microspheres were prepared by two different methods of polymer film grinding and a modified $\mathrm{W} / \mathrm{O} / \mathrm{W}$ multiple emulsion method as described in Chapters 2 and 4.

\subsubsection{PCL crystallites/spherulites preparation}

The spherulites were prepared by melting the PCL polymer in the plasticizer combination of ATEC and NMP $(9: 1 \mathrm{w} / \mathrm{w})$ under continuous stirring. Once the polymer was melted completely and mixed uniformly with the plasticizers, GMO was added slowly to above solution. The entire system was allowed to cool gradually at room temperature while undergoing stirring. The PCL spherulites begin to nucleate once the system is close to room temperature followed by larger crystallite or spherulite growth resulting in a turbid suspension. The flow chart for crystallite preparation is depicted in Figure 5-3.

\subsubsection{PCL crystallites/spherulites characterization}

Crystallites were characterized by polarized light microscopy, SEM and MDSC studies.

\subsection{Polarized light microscopy studies}

Polarized light microscopy was conducted on the injection vehicle system after equilibration with excess water or dissolution media to understand the crystalline structures formed by glycerol lipid. A Nikon Microphot FX microscope equipped with a polarizing filter. The images were captured with a high resolution Kodak DCS 460 color digital camera mounted on the microscope. Using a PowerPC G4, images were viewed on an Apple 20" Cinema Display monitor. The images were captured at a 200x magnification before and after equilibration of PCL spherulites with excess of water.

\subsection{Scanning electron microscopy studies}

SEM studies were done on the acetone cleaded spherulite samples using a FEI Philips environmental scanning electron microscope (ESEM). The samples were allowed 


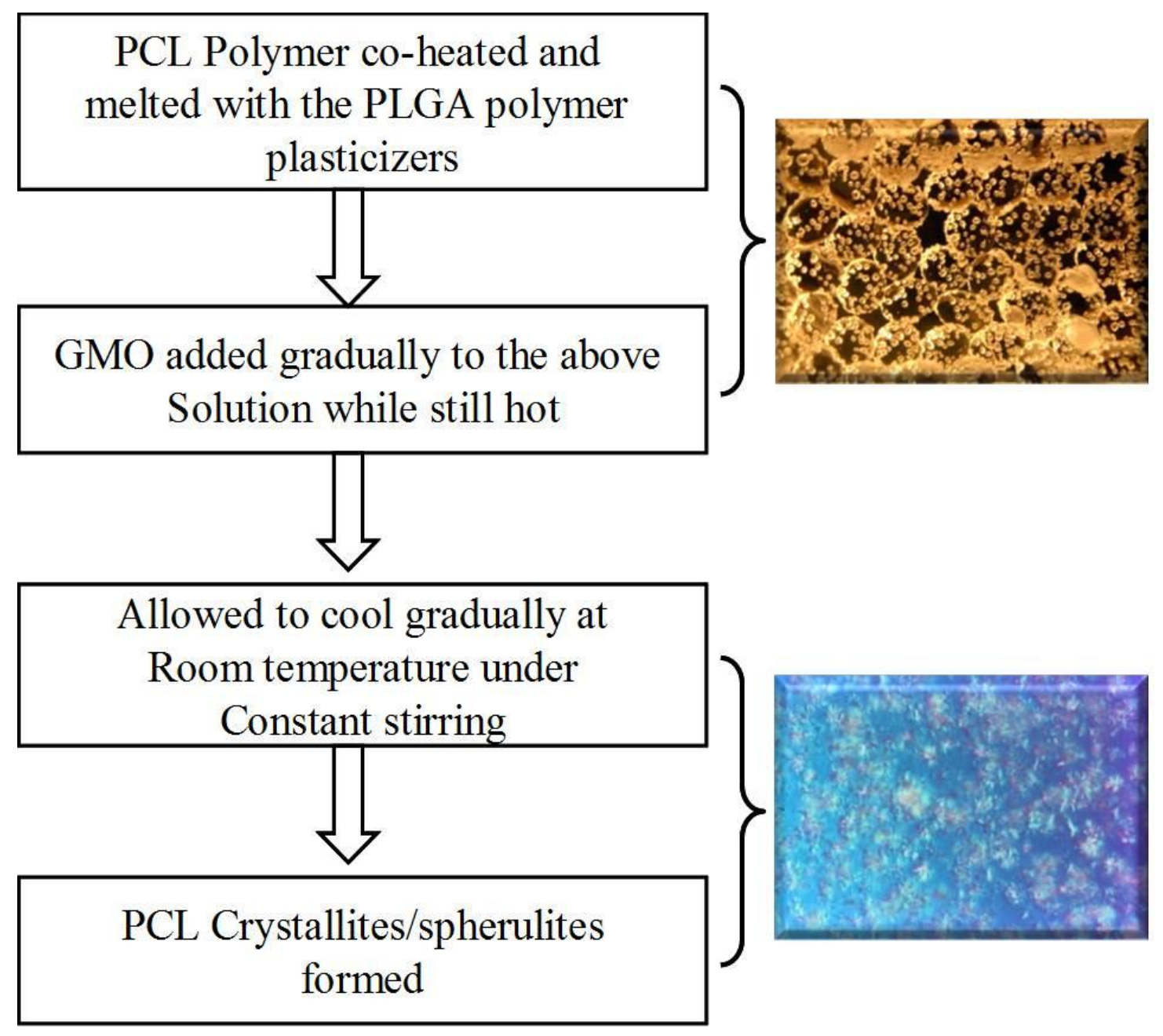

Figure 5-3. PCL Crystallite/spherulite preparation process. 
to air dry after removal of other components such as plasticizers and GMO with acetone, mounted onto SEM stubs and sputter coated with $40 \mathrm{~nm}$ AU/PD.

\subsection{Modulated differential scanning calorimetry studies}

MDSC studies were conducted on PCL spherulites and pure PCL polymer using a Thermal Analysis (TA) Q 2000 series DSC with autosampler. The parameters selected for the scan were: scanning rate of $3^{\circ} \mathrm{C} / \mathrm{min}$, modulation temperature amplitude of \pm $1.00^{\circ} \mathrm{C}$ and sinusoidal modulation of $60 \mathrm{sec}$.

\subsubsection{In vitro functionality testing}

Drug release was conducted in $0.012 \mathrm{M}$ PBS (pH 7.4) containing $0.02 \%$ sodium azide (Chapter 2). $10 \mathrm{ml}$ of dissolution media was taken in a $20 \mathrm{ml}$ glass scintillation vial. Vials were kept in a shaker incubator maintained at $37^{\circ} \mathrm{C}$ and $100 \mathrm{rpm}$ rotation. Formulations were placed at the bottom of glass vials and $2 \mathrm{ml}$ aliquots were taken at specific time points with replacement using fresh buffer. Drug content was determined using a RP-HPLC method. For large volume drug release studies, $150 \mathrm{ml}$ of PBS was taken in $250 \mathrm{ml}$ capacity glass cylindrical vessel (Figure 5-4) and $75 \mathrm{ml}$ of media was withdrawn for analysis of drug content at specific time points.

\subsubsection{Mechanistic studies}

The drug release from novel PLGA micro particulate: PCL crystallite formulation was evaluated mechanistically by comparing percent drug release with percent plasticizer (ATEC) release from the formulation. The plasticizer release during in vitro studies was determined by a RP-HPLC method that quantifies both drug and plasticizer in a single run. The HPLC method has been discussed in detail in Chapter 2.

\subsubsection{Mathematical modeling}

Seven different models were used to determine the drug release mechanism from the PLGA micro particulate: PCL crystallite formulation (Table 4-2) (Chapter 4). An excel add-in program, "DD Solver" was used for this purpose and $\mathrm{R}^{2}$ values were compared for different models.

\subsubsection{Viscosity and injection force measurement}

Viscosity of the novel PLGA micro particulate: PCL crystallite formulation was measured using a Brookfield DV- III Ultra programmable rheometer and the Rheocalc 3.2 software. Injection forces were determined using a Chatillon TCD-200 digital force 


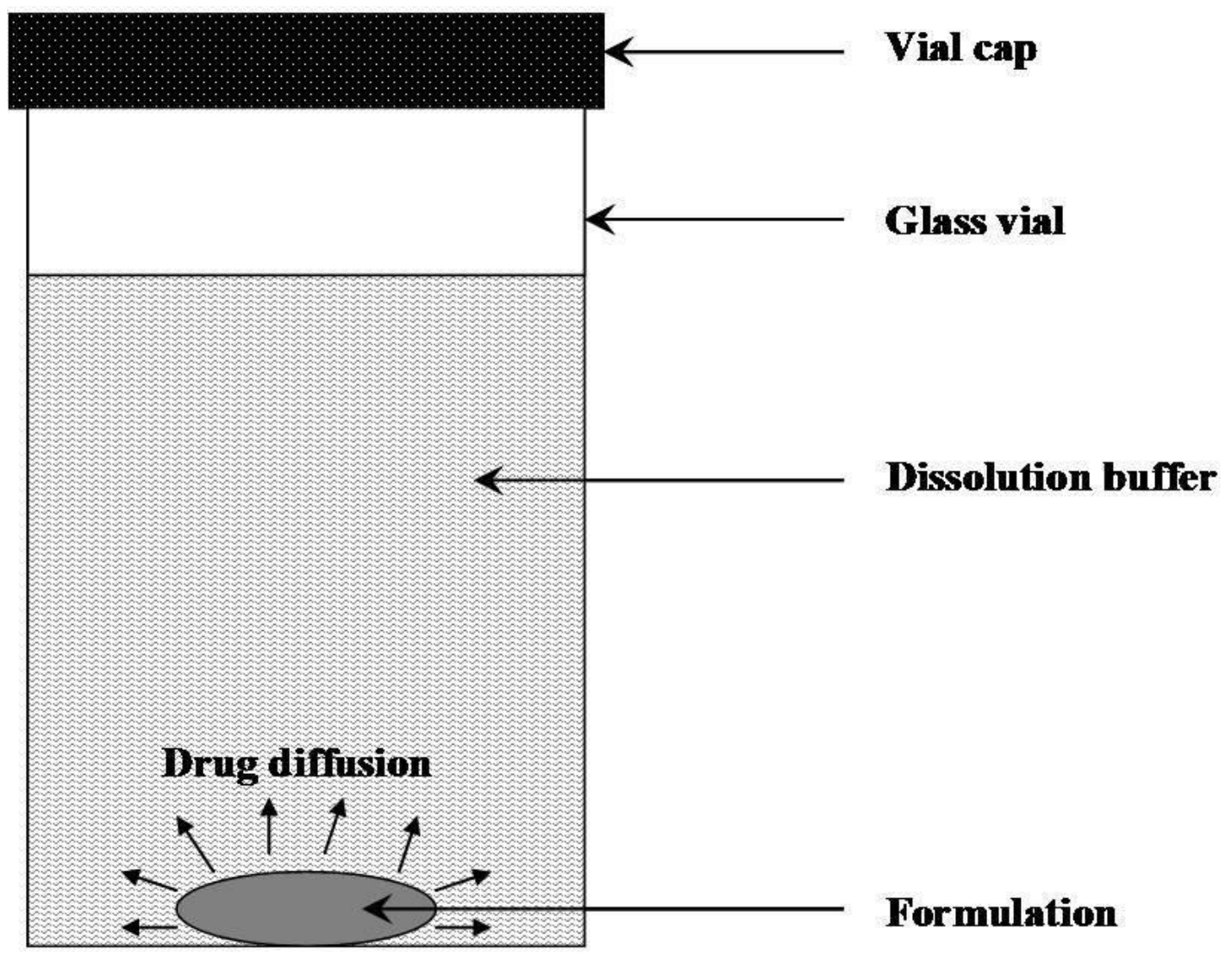

Figure 5-4. Experimental set up for in vitro drug release studies. 
tester connected to a computer and controlled by NEXYGEN FM series software. The determinations were done at three different PCL crystallite concentrations $(6 \%, 8 \%$ and $12 \% \mathrm{w} / \mathrm{w}$ ) in the injection vehicle.

\subsection{Results and Discussions}

5.3.1. PCL crystallites/spherulites characterization

\subsubsection{Polarized light microscopy studies}

Polarized microscopic images showed a birefringent pattern for the PCL crystallites, which is characteristic of many crystalline substances. The distance between individual crystallites was reduced upon contact and equilibration of the crystallites with excess of water (Figure 5-5). The crystallites exhibited the characteristic lamellae well known and studied for semi-crystalline polymers. ${ }^{144}$ A detailed microscopic analysis confirmed the coexistence of PCL crystallites/ spherulites and the lipid (GMO) microcrystalline structures (Figure 5-6). Together the two structures were hypothesized to control initial burst from PLGA micro particles, whereby the lipid microcrystalline structures could create a well enclosed shell/environment around the PLGA micro particles undergoing gelation in presence of plasticizer. The lipid microcrystalline structures can be varied based on the vehicle composition (plasticizer to GMO ratio) as discussed in Chapter 4 thereby resulting in a PCL crystallite network surrounding PLGA micro particles which ranges from a more flexible to a relatively closely packed network.

\subsubsection{Scanning electron microscopy studies}

SEM scans showed the presence of a distinct rough surface for the PCL crystallites together with numerous three dimensional projections or grooves on the crystallite surface (Figure 5-7). Distinct lamellar structure was observed at a higher magnification that correlates well with that observed with polarized microscopic examination. The individual crystallites were expected to fit well into each other with the help of these surface projections and grooves. This essentially results in formation of a tight barrier network around the PLGA micro particles that is responsible for controlling the initial drug release.

\subsubsection{Differential scanning calorimetry studies}

MDSC studies showed the characteristic melting point $\left(\mathrm{T}_{\mathrm{m}}\right)$ of the pure PCL polymer at around $60^{\circ} \mathrm{C}$ (Figure 5-8). This was in confirmation with other studies

reported on the melting behavior of PCL polymer. ${ }^{149}$ The melting point was observed as a sharp endotherm in the MDSC thermogram. The PCL crystallites/spherulites however 

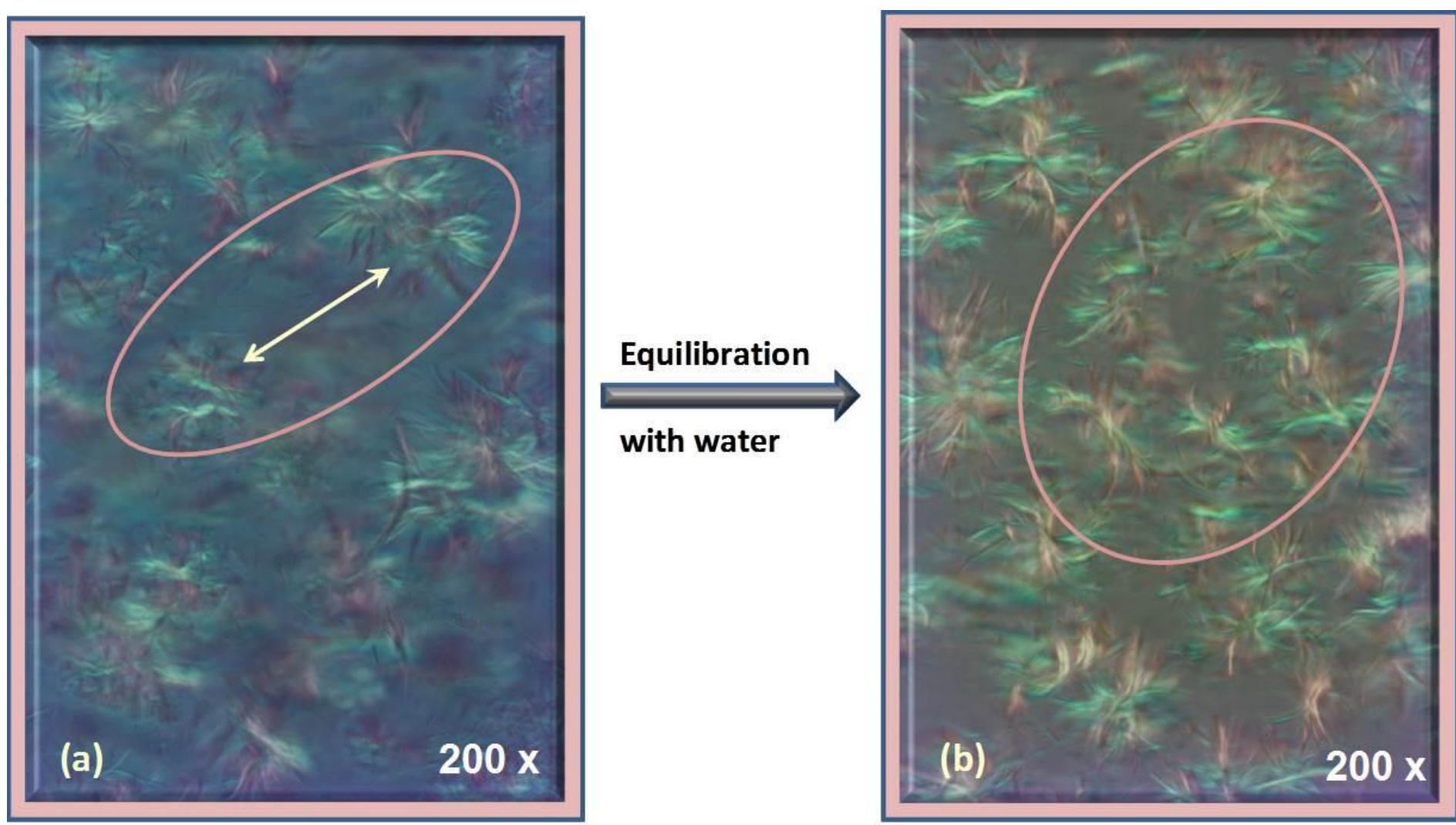

Figure 5-5. Polarized light microscopic images of PCL crystallites/spherulites (a) before; and (b) after equilibration with excess of water. 


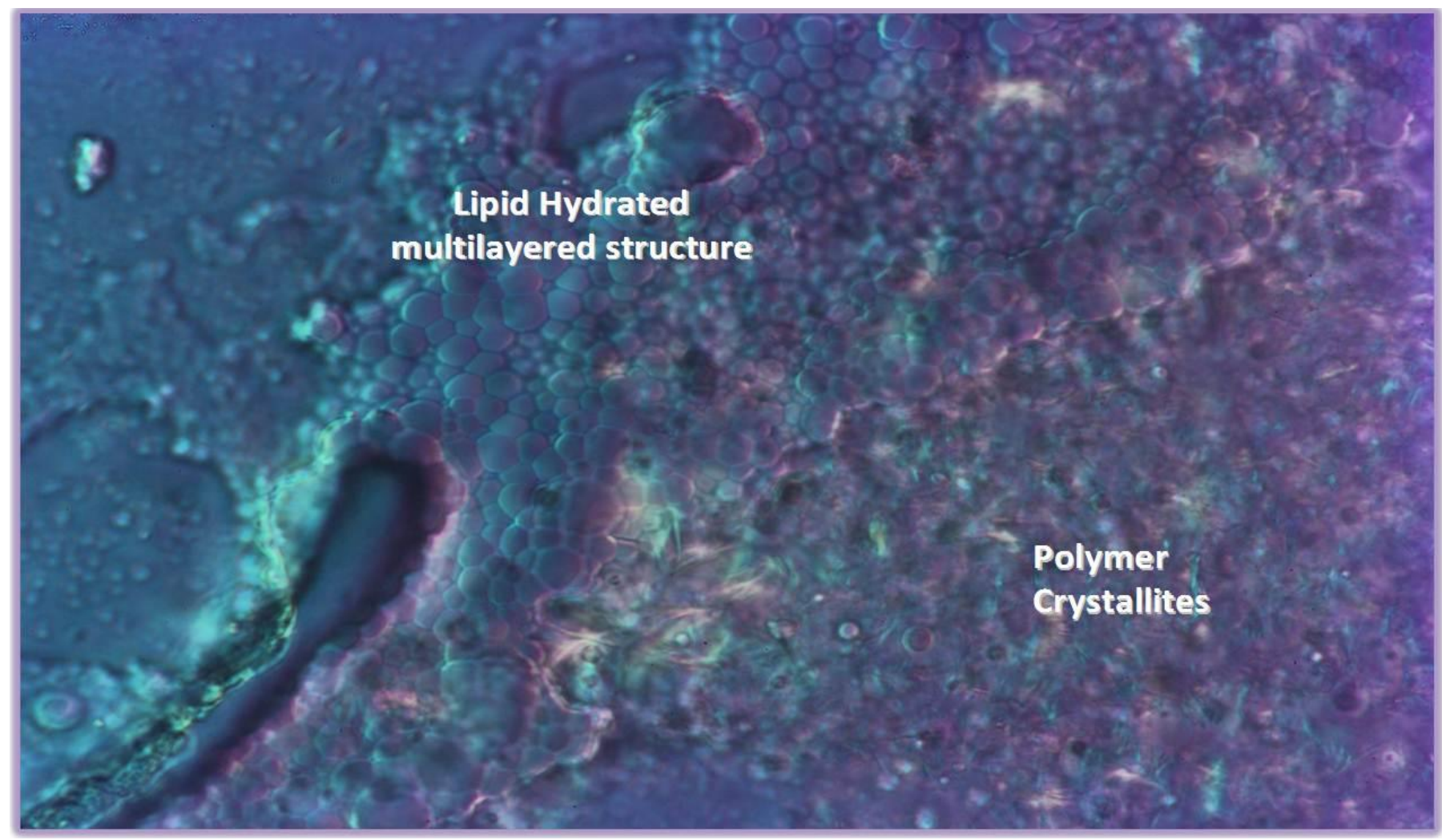

Figure 5-6. Polarized light microscopic images of co-existing PCL crystallites/spherulites and the glycerolipid microcrystalline structures. 

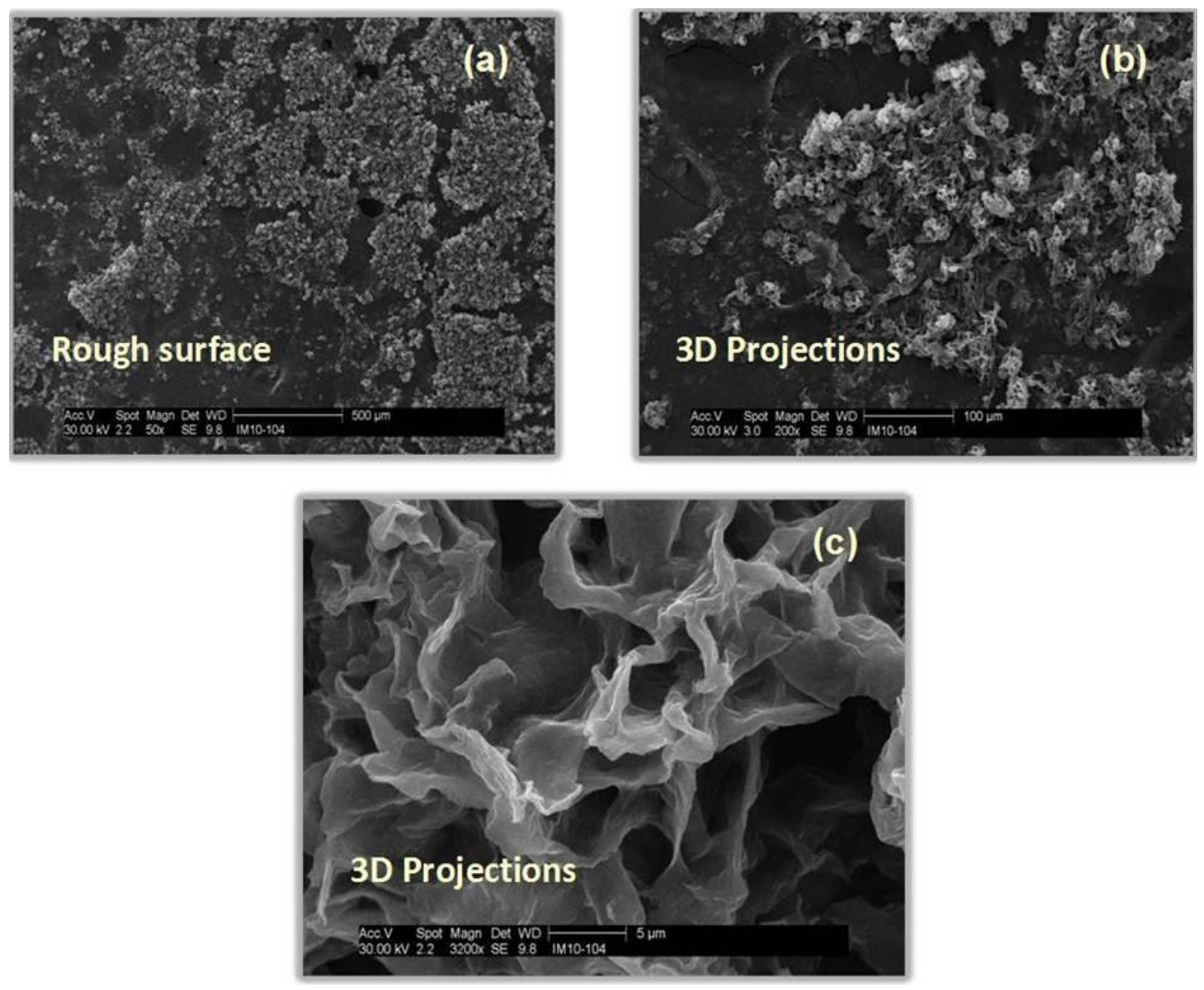

Figure 5-7. Scanning electron microscopic images of PCL crystallites at three different magnifications of (a) 50x; (b) 200x; and (c) 3200x. 


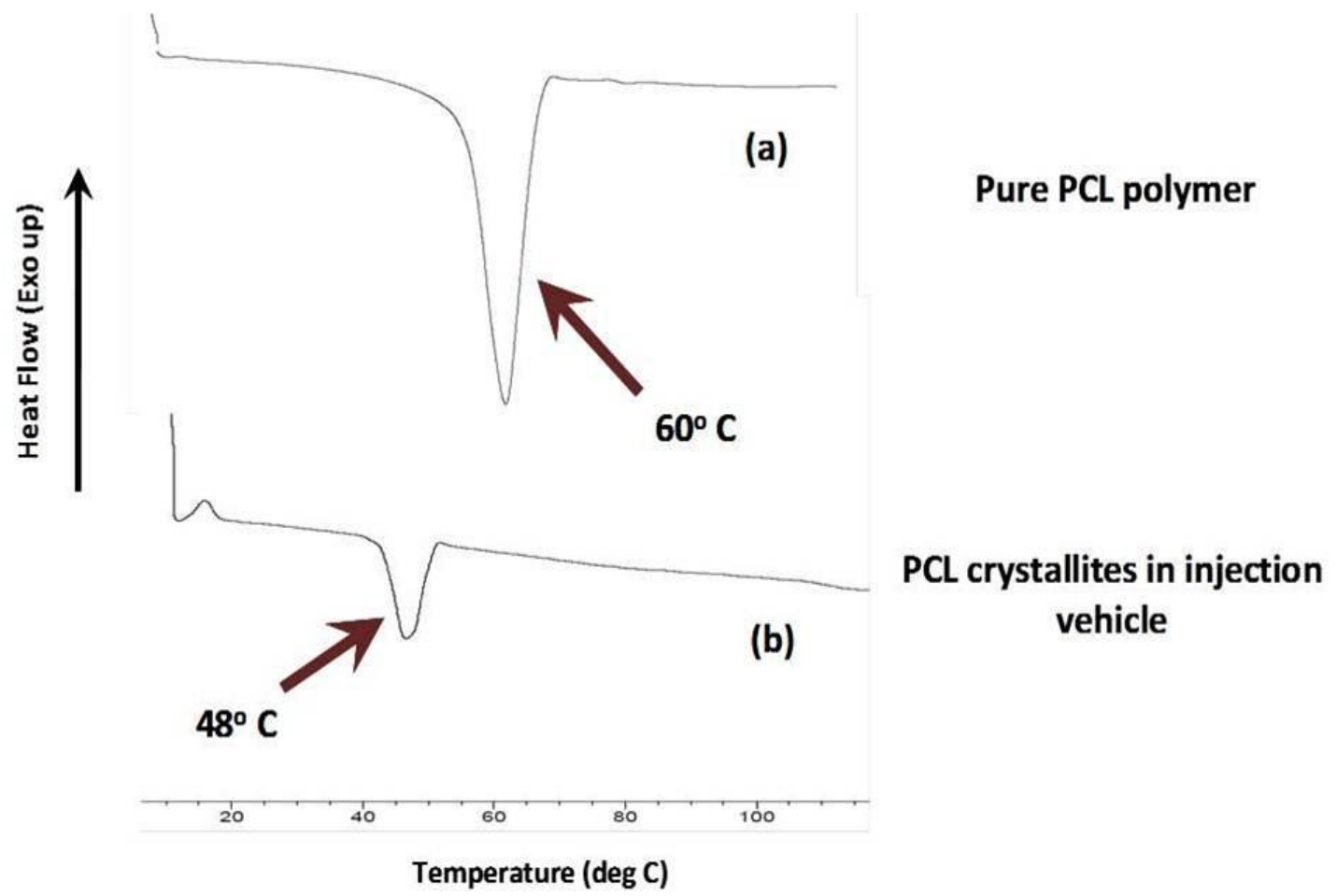

Figure 5-8. MDSC thermograms of (a) pure PCL polymer; and (b) PCL crystallites/spherulites. 
demonstrated a slight lowering of the polymer melting point from $60^{\circ} \mathrm{C}$ to around $48^{\circ} \mathrm{C}$. This was attributed to the reduction in melting point of the PCL polymer in presence of the other vehicle components i.e. the plasticizers and the lipid. The plasticizers and the lipid essentially acted as the impurities thereby causing a reduction in the melting point (colligative property) of the PCL polymer. The results, nonetheless, confirmed the crystalline nature of PCL crystallites/ spherulites in the injection vehicle.

\subsubsection{In vitro functionality studies}

\subsubsection{Effect of PCL crystallites concentration}

The results for the in vitro drug release studies demonstrated a significant reduction in initial drug burst upon increasing the PCL crystallite concentration in the injection vehicle (Figure 5-9). Increasing the crystallite concentration from $6 \% \mathrm{w} / \mathrm{w}$ to $12 \% \mathrm{w} / \mathrm{w}$ in injection vehicle resulted in a twofold reduction in the drug burst i.e. from about $20 \%$ to $10 \%$ drug release in first 24 hours. Further the reduction in drug burst was three fold in comparison to the formulation with no crystallites. This essentially confirmed our hypothesis of a tight network structure formed by the PCL crystallites around the PLGA micro particles once equilibrated with excess of water, which helped in reducing the initial drug release from the depot.

\subsubsection{Effect of dissolution media volume}

When placed subcutaneously, the implant is expected to release drug into its immediate vicinity (comprised of tissue fluid and cells), followed by passive diffusion or possibly facilitated transport into the cells and the vascular system before finally reaching the systemic circulation. ${ }^{101}$ The release of drug is therefore not directly into the systemic circulation. Among the various layers of subcutaneous tissue (pre-peritoneal, superficial subcutaneous and deep subcutaneous), normal blood flows have been found to range from 1.5 to $2.5 \mathrm{ml} / 100(\mathrm{~g} \mathrm{~min})^{-1} \cdot{ }^{150,151}$ At any one time, there is only a small volume of tissue fluid available for drug dissolution from the implant. To consider the effects of both the volume of tissue fluid available at the injection site and the continuous blood flow, it was imperative to conduct drug release studies at two different media volumes. Figure 5-10 shows the drug release profiles at two media volumes of 10 and $150 \mathrm{ml}$ at various PCL crystallite concentrations in the injection vehicle. The results showed similar dissolution profiles at different media volumes for $6 \%$ and $8 \% \mathrm{w} / \mathrm{w}$ crystallite concentrations in injection vehicle as further indicated by the respective $f_{2}$ values.

\subsubsection{Effect of PLGA end group on drug release}

PLGA micro particles prepared by film grinding method and two different end groups for PLGA polymer chains were compared in terms of surface adsorbed drug and 


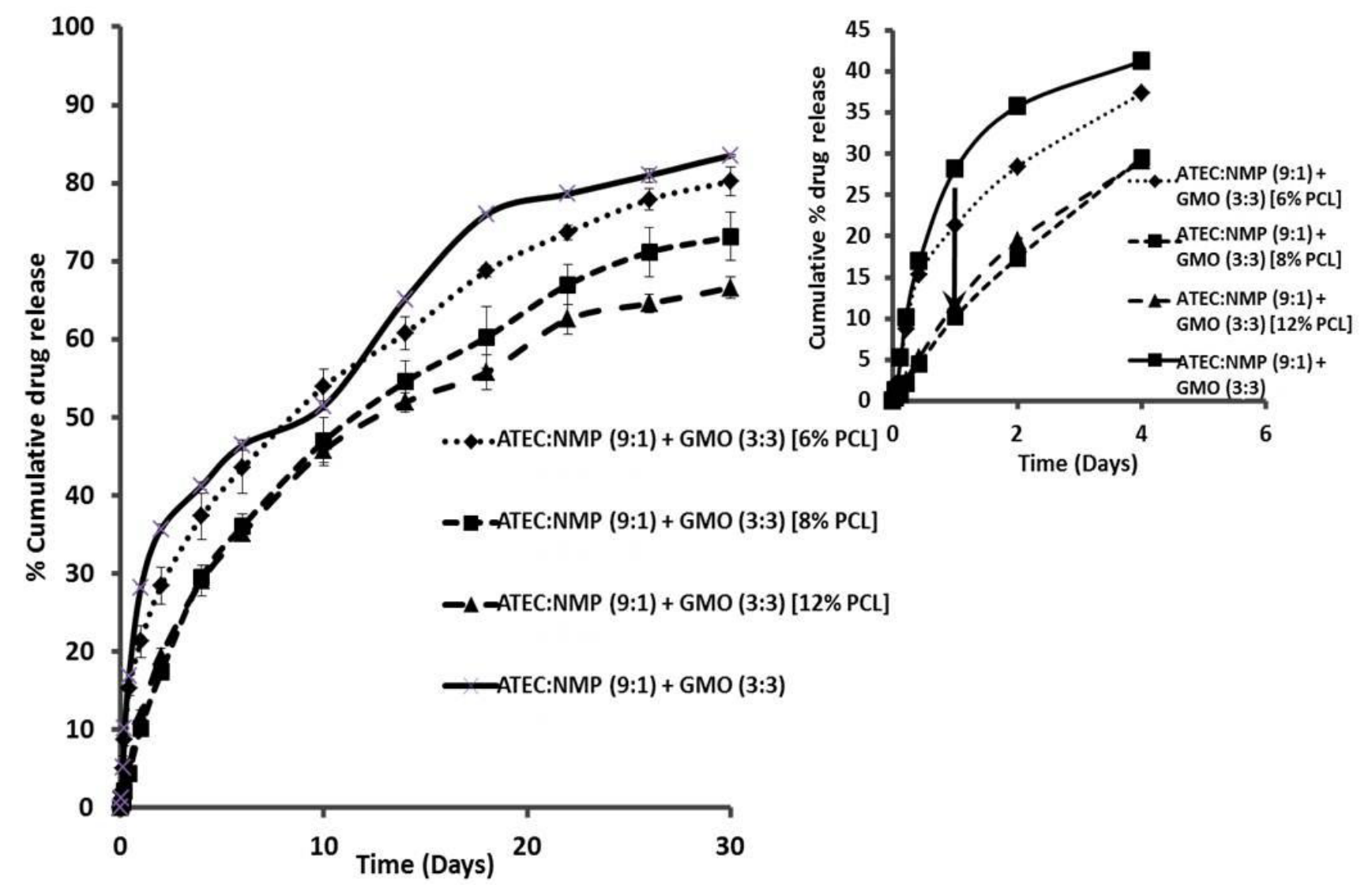

Figure 5-9. Effect of PCL crystallite concentration in injection vehicle on drug release.

Notes: Each data point represents an average of three measurements.

Standard deviation of three measurements is presented as error bars. 

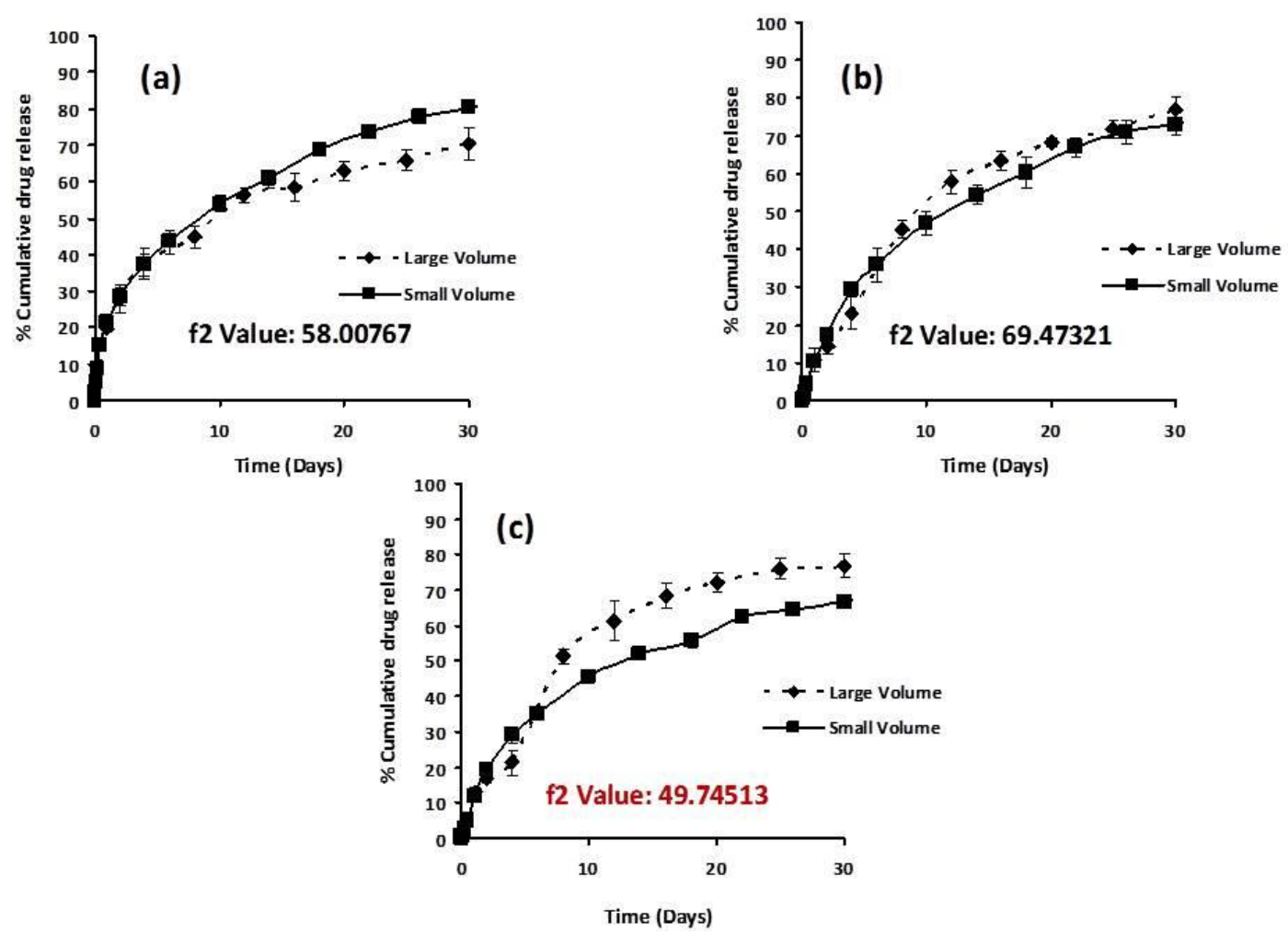

Figure 5-10. Effect of volume of dissolution media on drug release from PLGA micro particulate: PCL crystallite formulations at (a) $6 \% \mathrm{w} / \mathrm{w}$ PCL crystallite concentration; (b) $8 \% \mathrm{w} / \mathrm{w}$ PCL crystallite concentration; and (c) $12 \% \mathrm{w} / \mathrm{w}$ PCL crystallite concentration.

Notes: Each data point represents an average of three measurements. Standard deviation of three measurements is presented as error bars. 
overall drug release behavior. Results showed a significant difference (around two fold) in the amount of surface free drug on the two types of PLGA micro particles (Figure 5-11) with ester end group PLGA micro particles showing around $86 \%$ and acid end group micro particles showing around $44 \%$ of surface free drug. This was attributed to the ionic interactions between PLGA terminal carboxylic acid end group and the basic amino acids (arginine and histidine) of drug. Similar interactions between PLGA and leuprolide have been reported by Luan and Bodmeier. ${ }^{126}$ Interestingly, the initial drug release during first 24 hours was quite similar for the two types of PLGA micro particles (Figure 5-12). This was attributed again to the PCL crystallite network surrounding the PLGA micro particles, which controls the initial drug release from the micro particles. The drug release during the later part (day 15 to day 30), was however, controlled efficiently by the PLGA polymer type in the micro particles. The results showed a relatively faster drug release at the later stage from the acid end group PLGA micro particles than the ester end group micro particles. This can be well explained by the degradative nature of the two polymer types. The acid end group polymer, due to its greater propensity towards degradation, exhibits a faster drug release at the later stage.

\subsubsection{Effect of micro particle/microsphere morphology on drug release}

Figure 5-13 shows the drug release from two different micro particle morphologies, the film ground micro particles and the multiple emulsion microspheres. The initial drug release was reduced and became independent of the micro particle morphology in the presence of PCL crystallites in the injection vehicle. This further confirms the fact that PCL crystallites form a tight network structure around the PLGA micro particles such that the initial drug release becomes completely independent of other formulation factors i.e. PLGA polymer type and micro particle morphology. This presents a definite advantage over PLGA micro particle only based systems as the initial drug release is reduced and is well controlled by the PCL crystallite concentration alone.

\subsubsection{Effect of buffer concentration of dissolution media}

Once again PLGA micro particles with two different end groups (acid and ester) were compared in terms of drug release behavior at two different buffer concentrations of dissolution media. Results showed a relatively faster drug release from the capped PLGA micro particles (ester end group) at $1 / 20 \mathrm{M}$ buffer strength compared to $1 / 50 \mathrm{M}$ buffer, which was attributed to a greater drug solubilization in presence of a higher concentration of buffer ions (Figure 5-14). At higher concentrations of the buffering agents in the dissolution media, there is an increase in the drug super saturation levels. ${ }^{151}$ This increase in the degree of super-saturation levels, inhibit re-precipitation of drug resulting in a faster drug release from the formulation. The uncapped PLGA micro particles (acid end group) on the contrary, did not show any significant difference in drug release behavior at two different buffer concentrations. This can be described by the ionic interactions between the drug and polymer that are sufficient to overcome the increased solubilization caused by high concentration of the buffer. 


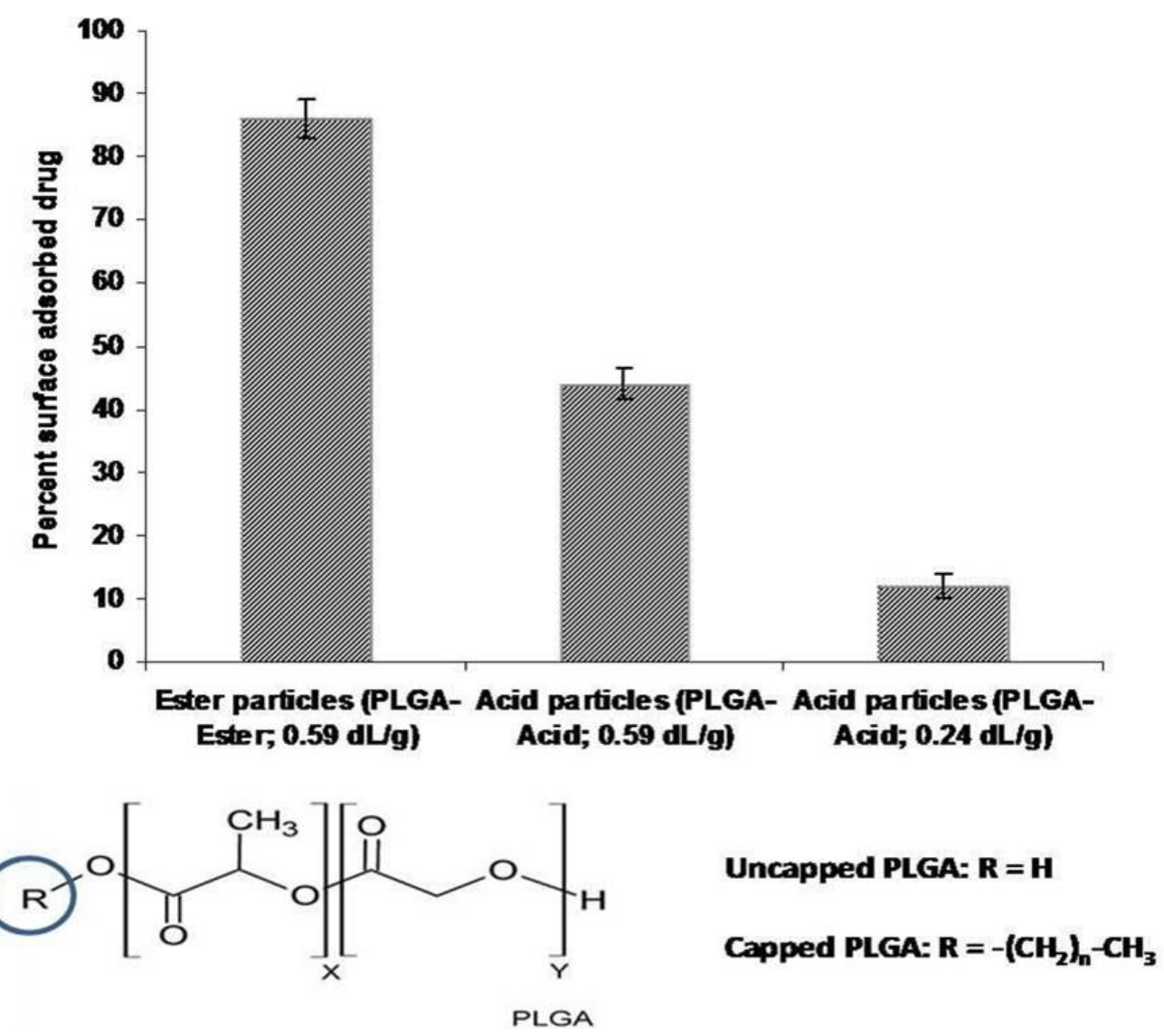

Figure 5-11. Effect of PLGA end group on amount of surface adsorbed drug on PLGA micro particles.

Notes: Each data point represents an average of three measurements.

Standard deviation of three measurements is presented as error bars. 


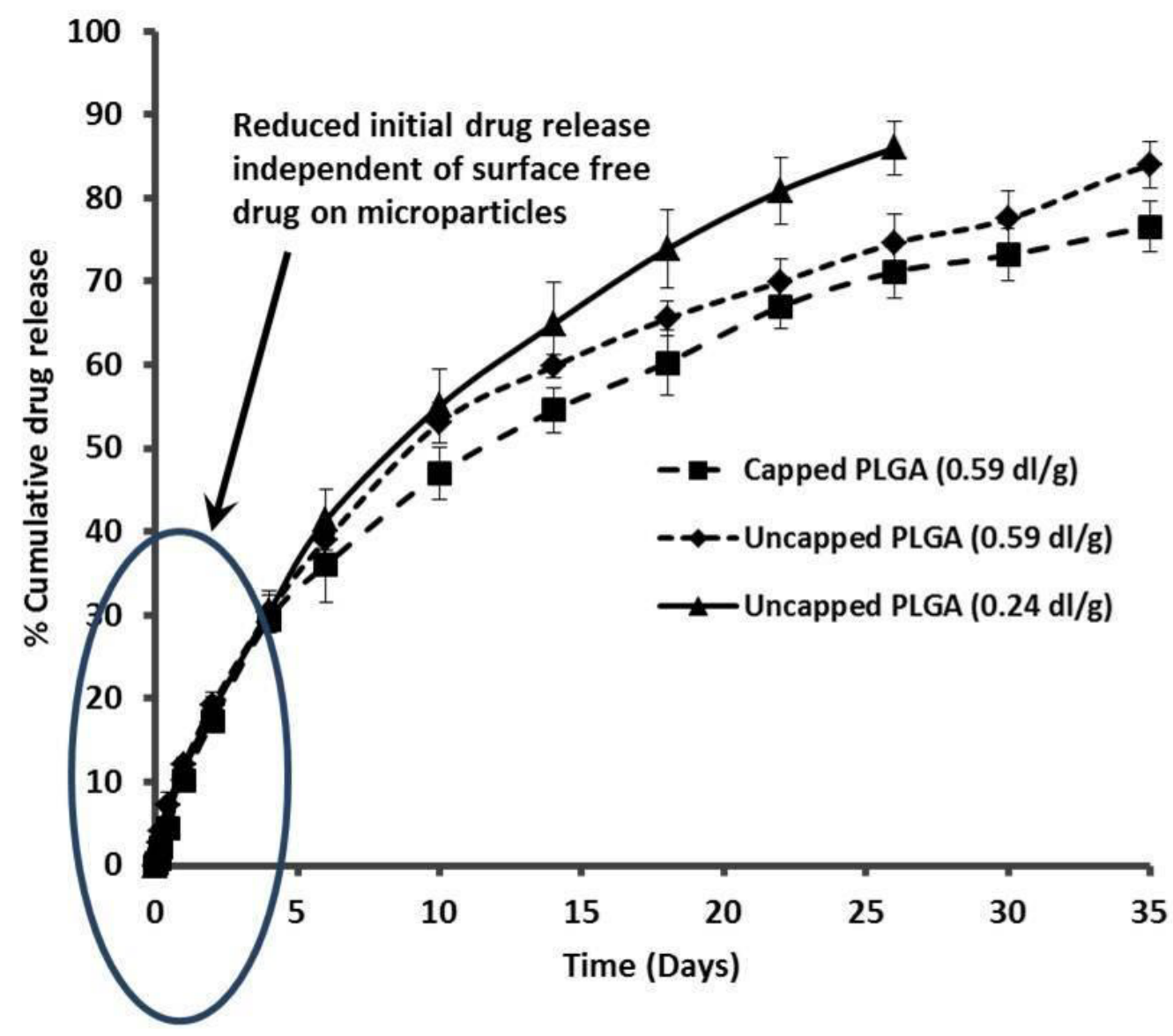

Figure 5-12. Effect of PLGA end group on drug release from PLGA micro particulate: PCL crystallite formulation.

Notes: Each data point represents an average of three measurements. Standard deviation of three measurements is presented as error bars. 


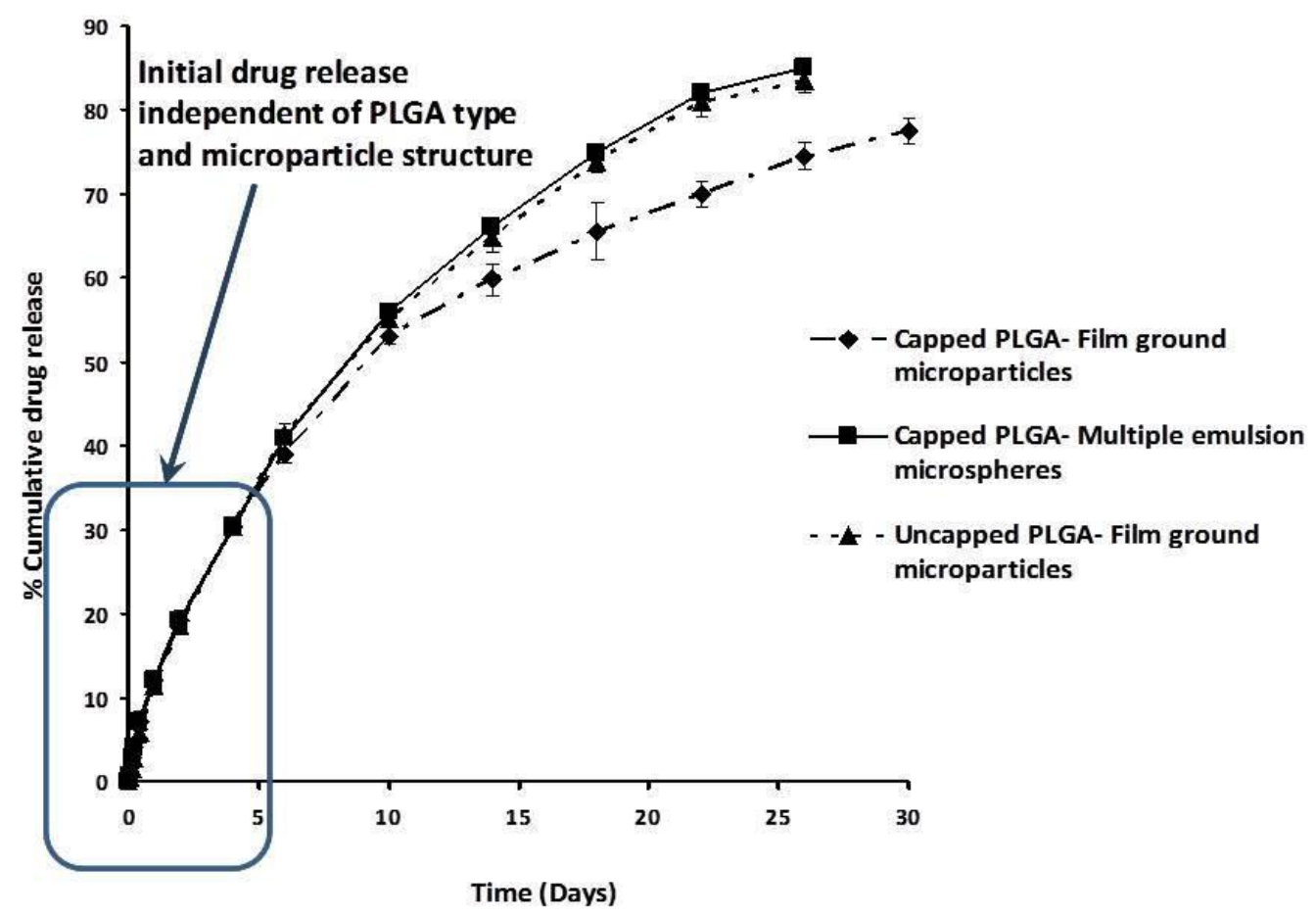

Figure 5-13. Effect of PLGA micro particle morphology on drug release from PLGA micro particulate: PCL crystallite formulation.

Notes: Each data point represents an average of three measurements. Standard deviation of three measurements is presented as error bars. 

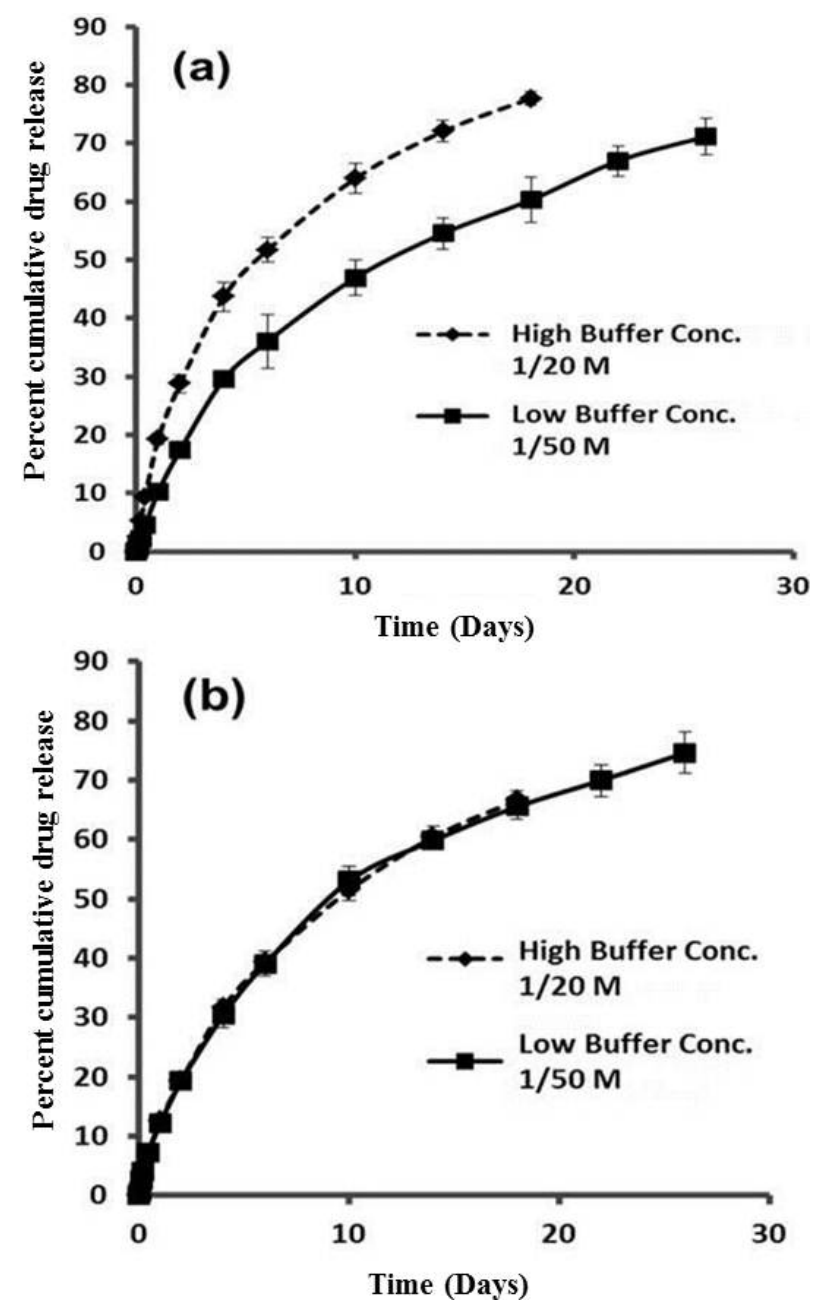

Figure 5-14. Effect of buffer strength on drug release from PLGA micro particulate:

PCL crystallite formulation having PLGA polymer with (a) ester end group; and (b) acid end group.

Notes: Each data point represents an average of three measurements.

Standard deviation of three measurements is presented as error bars. 


\subsubsection{Mechanistic studies}

Percent drug release was compared with the percent plasticizer (ATEC) released from the formulations over the course of in vitro dissolution studies. The results showed a higher cumulative percent drug release per unit solvent/plasticizer released for smaller volume $(10 \mathrm{ml})$ dissolution conditions at various PCL crystallite concentrations compared to the larger volume dissolution conditions (Figure 5-15). This had a direct correlation with the overall sink conditions in the two media volumes. Larger media volumes provided greater sink conditions for the plasticizer thereby increasing the rate of plasticizer release compared to the smaller media volumes. Nonetheless, the overall trend between different crystallite concentrations was similar between the two dissolution media volumes (Figure 5-16) indicating solvent diffusion as the major factor controlling drug release rate. Essentially, the initial plasticizer released was lower at higher crystallite concentrations for the same amount of drug released (marked as * in Figure 5-16) resulting in a relatively greater percentage of plasticizer trapped inside the depot at higher crystallite concentrations. This could be attributed to relatively faster crystallite aggregation and faster depot formation at higher crystallite concentrations.

\subsubsection{Mathematical modeling}

The $\mathrm{R}^{2}$ values determined using various mathematical equations are presented in Table 5-1 with the Peppas and Sahlin equation showing the highest $\mathrm{R}^{2}$ value indicating the best fit at various PCL crystallite concentrations and two different dissolution media volumes. Peppas and Sahlin equation is of special interest as it helps in determining the relative contributions of the diffusion based drug release process and the erosion based release process simply by computation of the diffusion and relaxation coefficients. Ratio between the relaxation and diffusion coefficients were calculated at different time points throughout the drug release studies and results were plotted with respect to time (Figure 5-17). Constantly decreasing values for the ratio between relaxation and diffusion coefficients with respect to time indicated an increasingly diffusion based drug release process over time. Further, the values were compared as a function of PCL crystallite concentration and the results showed decreasing values with increasing crystallite concentrations. This indicated an overall greater extent of drug diffusion at higher PCL crystallite concentrations. As mentioned under mechanistic studies, the higher percentage of trapped plasticizer at relatively higher crystallite concentrations could be responsible for a greater diffusion based drug release.

\subsubsection{Viscosity and injectability studies}

The viscosity of formulation containing $6 \% \mathrm{w} / \mathrm{w}$ PCL crystallites in the injection vehicle was significantly higher than without any crystallites (Figure 5-18). This could mean greater injection forces and a serious loss of injectability for crystallite based formulations. Interestingly, however significantly lower injection forces were maintained for more than 30 min once the PLGA micro particles were dispersed in the crystallite 

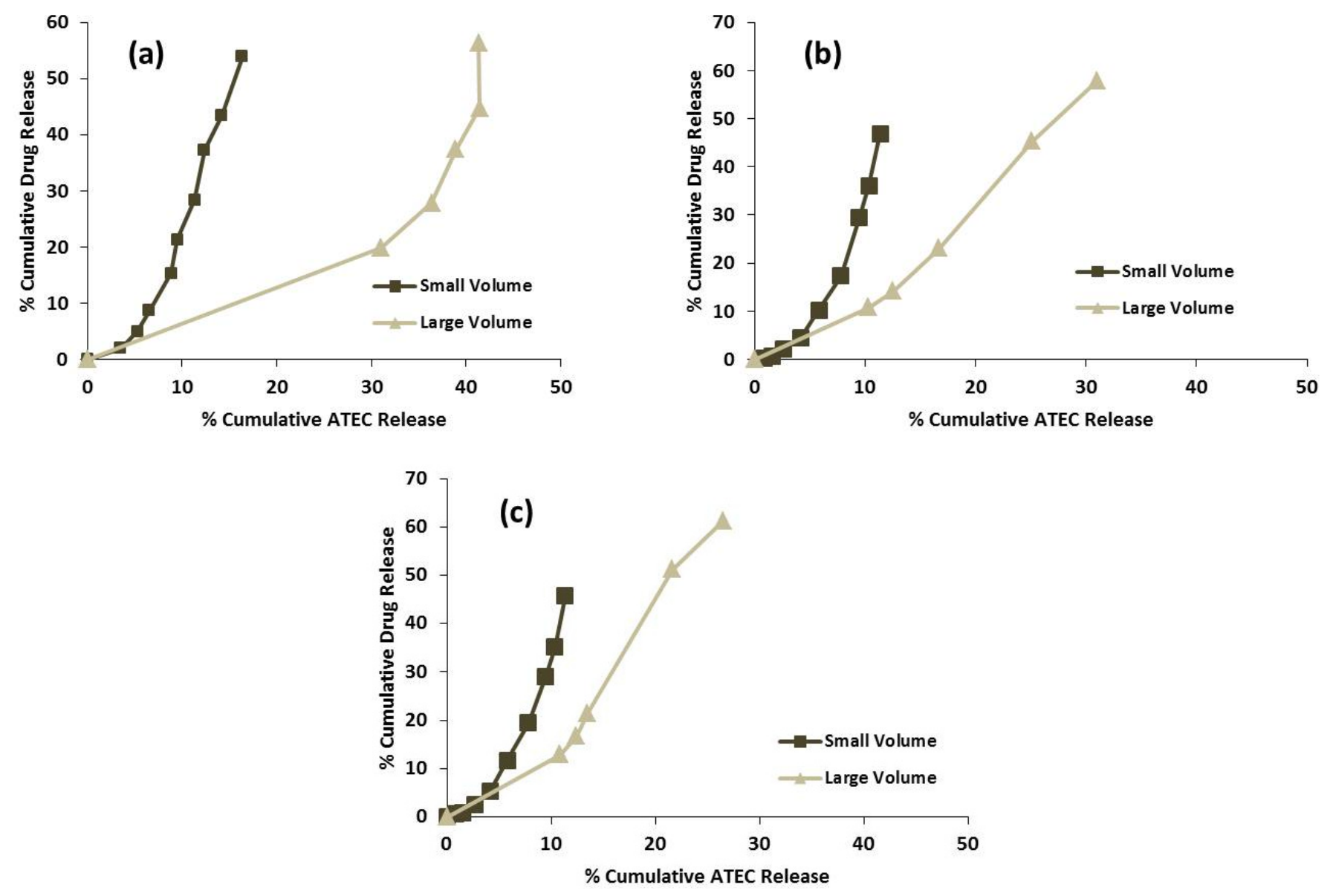

Figure 5-15. Mechanistic evaluation and correlation between percent drug and percent plasticizer release at two different dissolution media volumes and at PCL crystallite concentrations of (a) $6 \% \mathrm{w} / \mathrm{w}$; (b) $8 \% \mathrm{w} / \mathrm{w}$; and (c) $12 \% \mathrm{w} / \mathrm{w}$ in injection vehicle. 

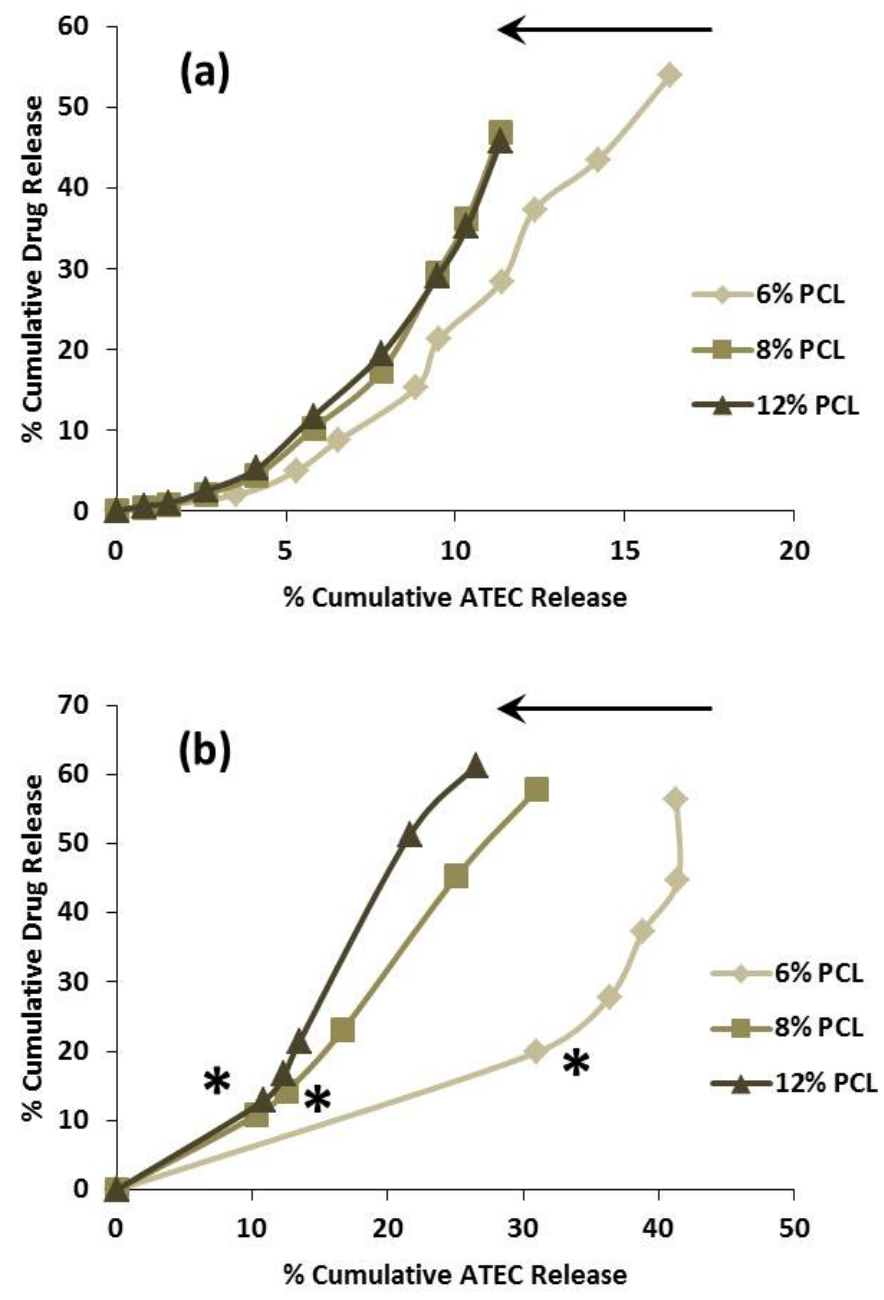

Figure 5-16. Evaluation of drug release mechanism at various PCL crystallite concentrations and two different dissolution media volumes of (a) $10 \mathrm{ml}$; and (b) $150 \mathrm{ml}$. 
Table 5-1. Calculated $\mathrm{R}^{2}$ values from various mathematical models.

\begin{tabular}{|c|c|c|c|c|c|c|}
\hline \multirow[t]{2}{*}{ Formulation } & \multicolumn{6}{|c|}{$\mathbf{R}^{2}$ value } \\
\hline & Zero order & First order & Higuchi & $\begin{array}{l}\text { Korsenmeyer } \\
\text { Peppas }\end{array}$ & $\begin{array}{l}\text { Peppas } \\
\text { Sahlin }\end{array}$ & Kopcha \\
\hline $\begin{array}{l}6 \% \text { crystallites Large } \\
\text { volume }\end{array}$ & 0.3991 & 0.7544 & 0.9305 & 0.9938 & 0.997 & 0.9963 \\
\hline $\begin{array}{l}6 \% \text { crystallites Small } \\
\text { volume }\end{array}$ & 0.7182 & 0.912 & 0.9817 & 0.9961 & 0.998 & 0.998 \\
\hline $\begin{array}{l}8 \% \text { crystallites Large } \\
\text { volume }\end{array}$ & 0.7864 & 0.972 & 0.9728 & 0.9738 & 0.9943 & 0.9728 \\
\hline $\begin{array}{l}8 \% \text { crystallites Small } \\
\text { volume }\end{array}$ & 0.8553 & 0.9685 & 0.9898 & 0.9904 & 0.9982 & 0.9798 \\
\hline $\begin{array}{l}12 \% \text { crystallites Large } \\
\text { volume }\end{array}$ & 0.7261 & 0.9586 & 0.955 & 0.955 & 0.9884 & 0.9569 \\
\hline $\begin{array}{l}12 \% \text { crystallites Small } \\
\text { volume }\end{array}$ & 0.8065 & 0.934 & 0.9872 & 0.9878 & 0.9978 & 0.9903 \\
\hline
\end{tabular}




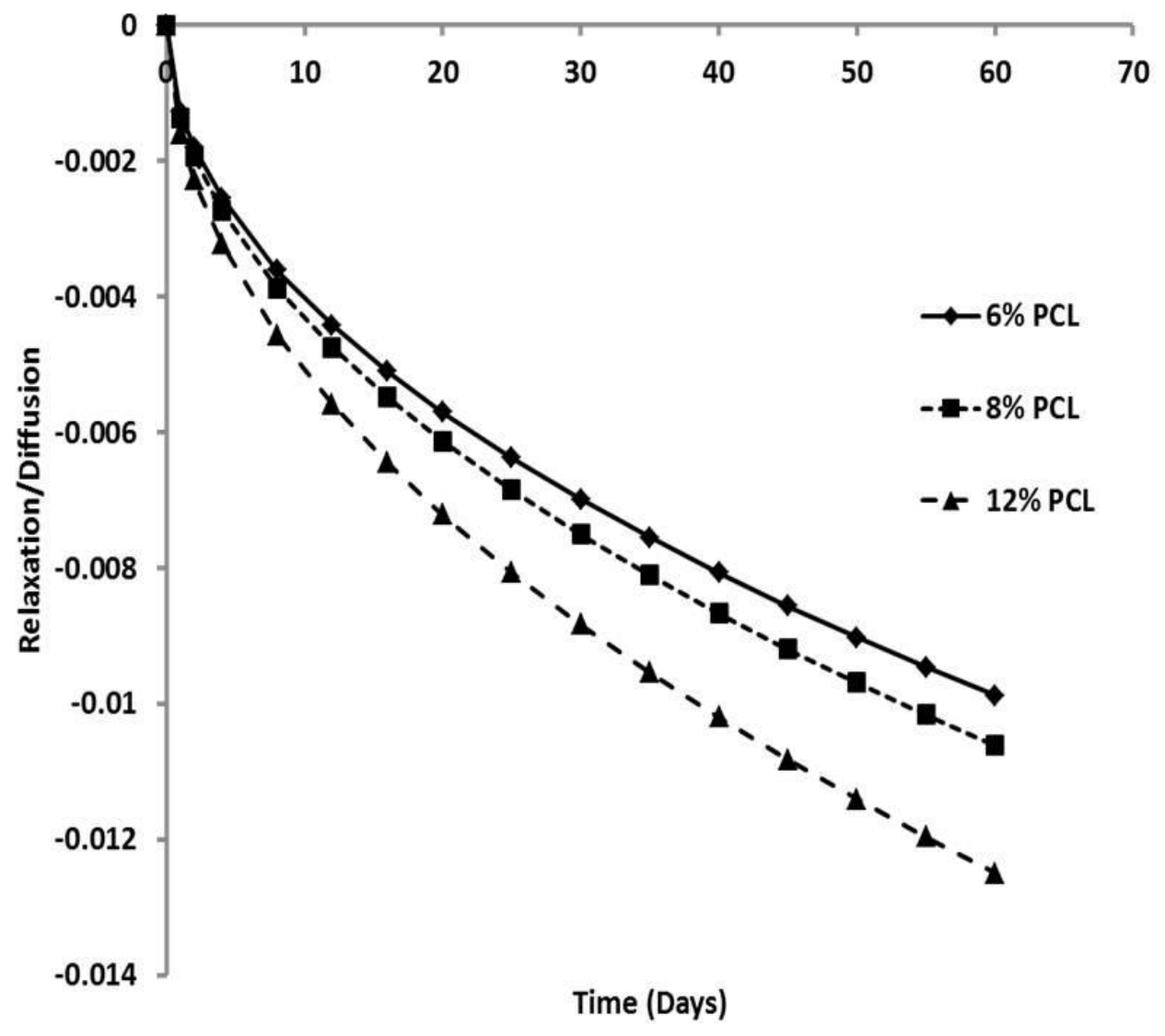

Figure 5-17. Relative relaxation and diffusion coefficients for drug release at various PCL crystallite concentrations. 
(a)
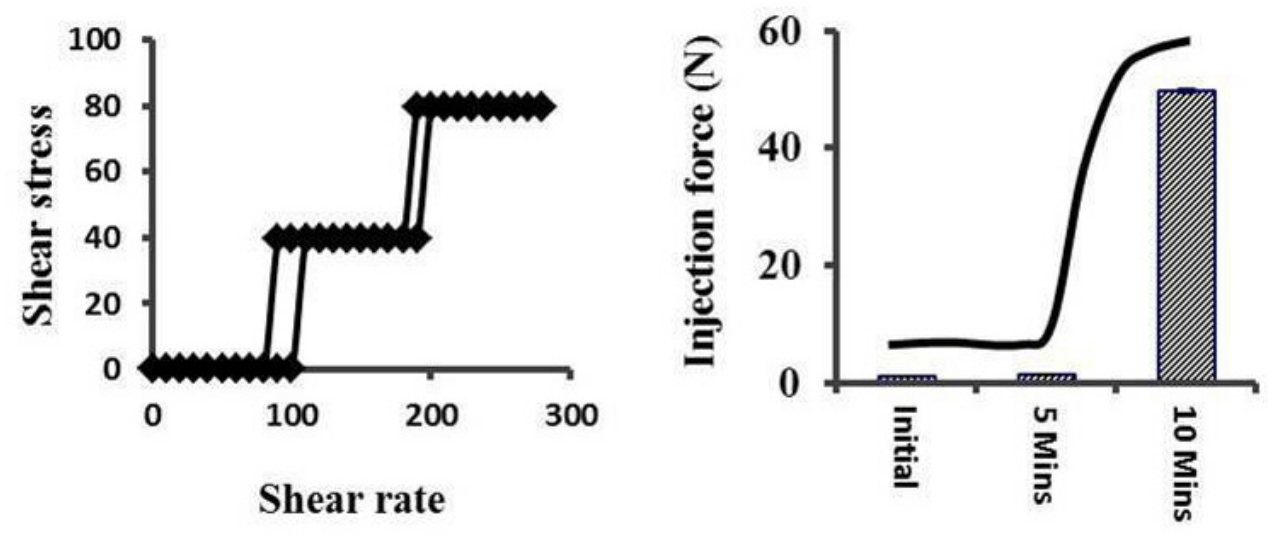

Viscosity $36.8 \mathrm{cps}$

(b)
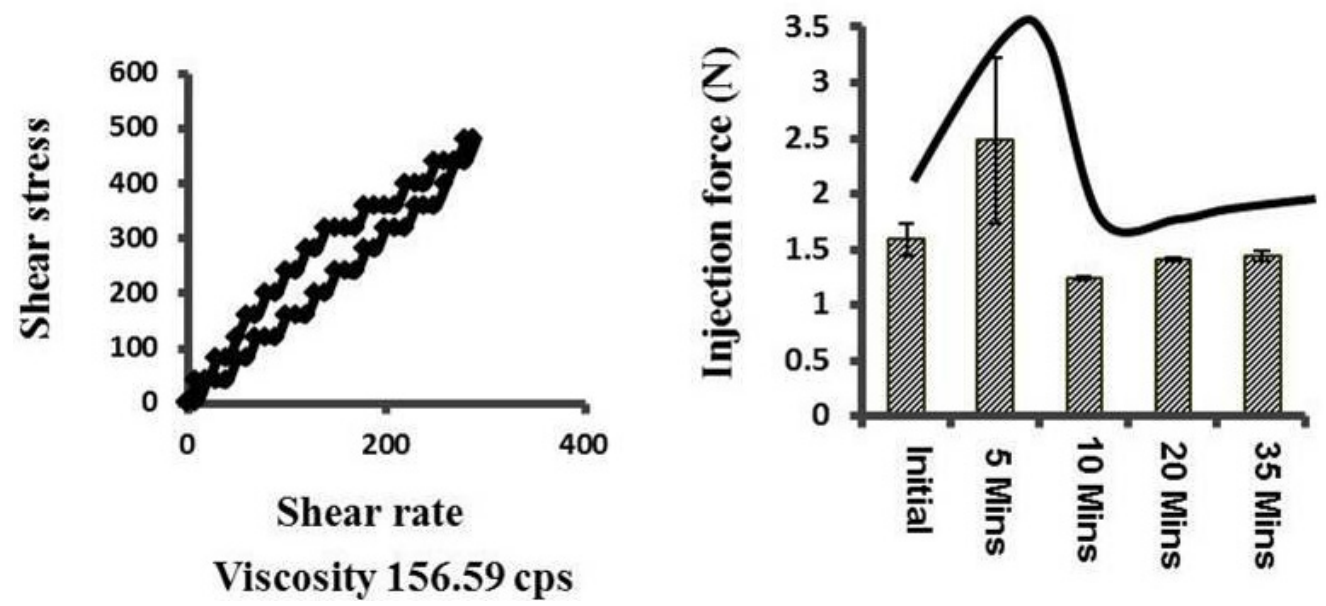

Figure 5-18. Viscosity and injection forces of injection vehicle in (a) absence; and (b) presence of PCL crystallites. 
containing formulation compared to the one with no crystallites. Injection forces of less than $3.5 \mathrm{~N}$ could be maintained for more than $30 \mathrm{~min}$ in presence of crystallites than in their absence where the injection forces increased up to $60 \mathrm{~N}$ within 10 min after mixing the contents of the formulation. This is attributed to the unique morphology of PCL crystallites or spherulites, which due to their thixotropic nature, were able to keep PLGA micro particles in s stable suspension system. The spherulites further demonstrate the much desired shear thinning behavior that provides an ease of injectability and administration. Furthermore, although there was an increase in overall viscosity of injection vehicle upon increasing the crystallite concentration, there was no significant increase in the injection force (Figure 5-19).

\subsection{Conclusion}

The study showed the unique capability of PCL crystallites or spherulites to cause a significant reduction in the initial drug burst and modify tri-phasic release pattern, make the initial drug release independent of PLGA polymer type and micro particle morphology and provide the desired shear thinning and thixotropic characteristics to the formulation. The drug release rate during initial phase was well controlled by the PCL concentration and that during later stages was well controlled and modulated by the PLGA polymer type. Dissolution media volume showed negligible effect on drug release from the novel PLGA micro particulate: PCL crystallite formulation. The PCL crystallites imparted a thixotropic nature to the injection vehicle, which then further provided excellent structural stability to the formulation for at least 30 minutes prior to administration with no significant change in drug release. The drug was released mainly by either a solvent/plasticizer controlled diffusion process or by a pore based diffusion process. 

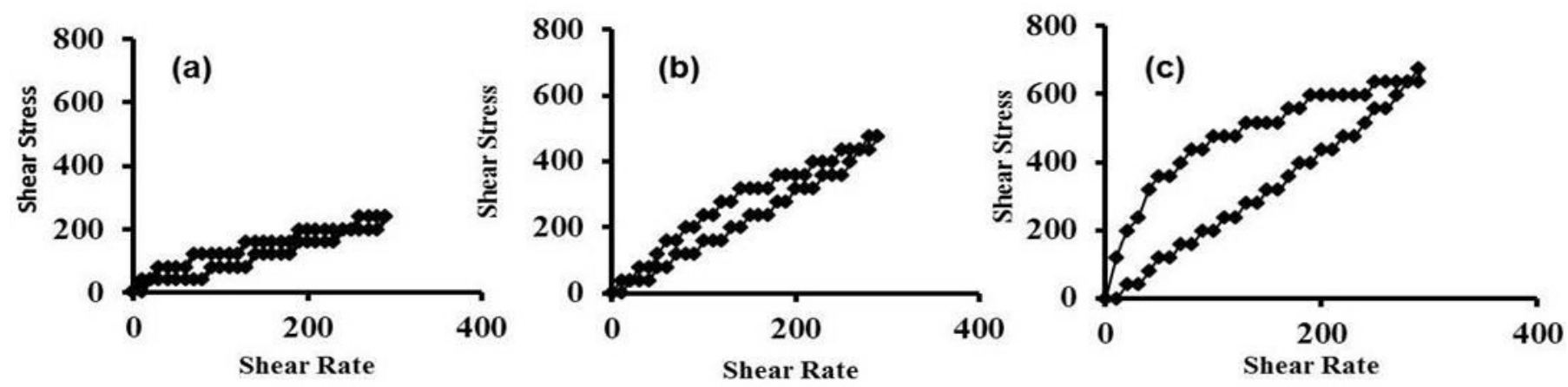

Viscosity $84.46 \mathrm{cp}$

Viscosity $156.59 \mathrm{cp}$

Viscosity $197.09 \mathrm{cp}$
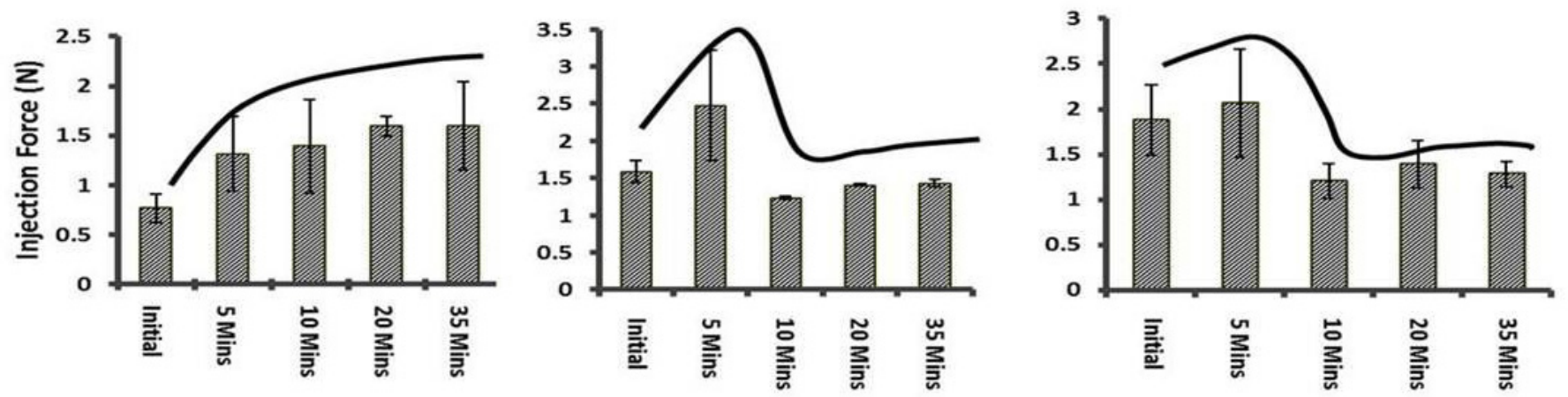

Figure 5-19. Viscosity and injection forces of injection vehicle at various PCL crystallite concentrations of (a) 6\% w/w; (b) $8 \% \mathrm{w} / \mathrm{w}$; and (c) $12 \% \mathrm{w} / \mathrm{w}$. 


\section{CHAPTER 6. EVALUATION OF BIODEGRADABILITY AND BIOCOMPATIBILITY OF PLGA MICRO PARTICLE: POLYCAPROLACTONE CRYSTALLITE BASED IN SITU IMPLANT}

\subsection{Introduction}

Tremendous progress has been made in recent years pertaining to the development of various implantable devices such as drug-eluting stents, ${ }^{152-158}$ artificial organs, ${ }^{158-160}$ biosensors, ${ }^{161,162}$ catheters, ${ }^{163}$ scaffolds for tissue engineering, ${ }^{164-166}$ heart valves ${ }^{167,168}$ etc. The devices have been employed for several different purposes including drug delivery. The biggest concern with any of such devices is their biocompatibility whereby different immune reactions are triggered in the body. Various polymeric systems have been used till date for drug delivery applications including chitosan polymer, ${ }^{169-171}$ alginate, ${ }^{172}$ collagen, ${ }^{173,174}$ hyaluronic acid, ${ }^{175}$ dextran, ${ }^{176,177}$ PLGA,${ }^{45,47,178-182}$ PEG, ${ }^{183,184}$ and PVA ${ }^{185-187}$ based systems etc.

Biocompatibility is generally defined as the ability of a biomaterial or medical device to perform with an appropriate host response in a specific application. Different biomaterials may trigger host responses of various extents, which further undergo resolution in various time dependent manners. The process of the implant placement into the tissue causes an injury that essentially initiates a cascade of the cellular responses leading to wound healing. ${ }^{188}$ The sequence of events involved in the immune response following the implantation procedure is as follows:

- Injury

- Blood-material interactions

- Provisional matrix formation

- Acute inflammation

- Chronic inflammation

- Granulation tissue

- Foreign body reaction

- Fibrosis/fibrous tissue development

\subsubsection{Blood material interactions}

Blood material interactions are involved in the early responses to injury where blood comes in contact with the biomaterial for first time and the process is intimately linked to the inflammatory response process. ${ }^{189-192}$ The process eventually leads to thrombus/clot formation thereby resulting in an activation of the extrinsic and intrinsic 
coagulation systems, the complement system, the fibrinolytic system, the kinin-generating system, and platelets.

\subsubsection{Provisional matrix formation}

Within minutes to hours of implantation procedure, matrix formation around the implanted biomaterial takes place. This provisional matrix consists of fibrin, produced by activation of the coagulative and thrombosis systems, and inflammatory products, released by the complement system, activated platelets, inflammatory cells, and endothelial cells. ${ }^{193-195}$ The provisional matrix essentially acts towards the wound healing process by providing a rich source of substances such as mitogens, chemo-attractants, cytokines, and growth factors. ${ }^{196-201}$ Provisional matrix consists of:

- Fibrin, produced by activation of the coagulation and thrombosis systems.

- Inflammatory products released by the complement system, activated platelets, inflammatory cells and endothelial cells.

\subsubsection{Acute inflammatory response}

In general, the biocompatibility of a material with tissue has been described in terms of the acute and chronic inflammatory responses and of the fibrous capsule formation that is seen over various time periods following implantation. ${ }^{202,203}$ The process involves emigration of neutrophils (polymorphonuclear leukocytes, PMNs) and other motile white cells from the blood vessels to the perivascular tissues and the injury site with the process lasting from minutes to days. ${ }^{204-206}$ Once the leukocytes get localized at the site of injury, the neutrophils and macrophages gets activated thereby releasing the enzymes. The major role of the neutrophil in acute inflammation is to phagocytose microorganisms and foreign materials. The major clinical signs for acute inflammation are:

- Redness (rubor)

- Swelling (tumor)

- Pain (dolor)

- Heat (calor)

Swelling during acute inflammation is mainly due to an increase in the pressure difference between capillary and external tissue bed and further due to an increase in the permeability of blood capillaries. The blood capillaries have low permeability under normal conditions with tight junctioned endothelium and the permeability increases under inflamed conditions. This results in permeability of larger molecules into the tissue at higher rates. Figure 6-1 shows the hypothetical representation of the endothelial 

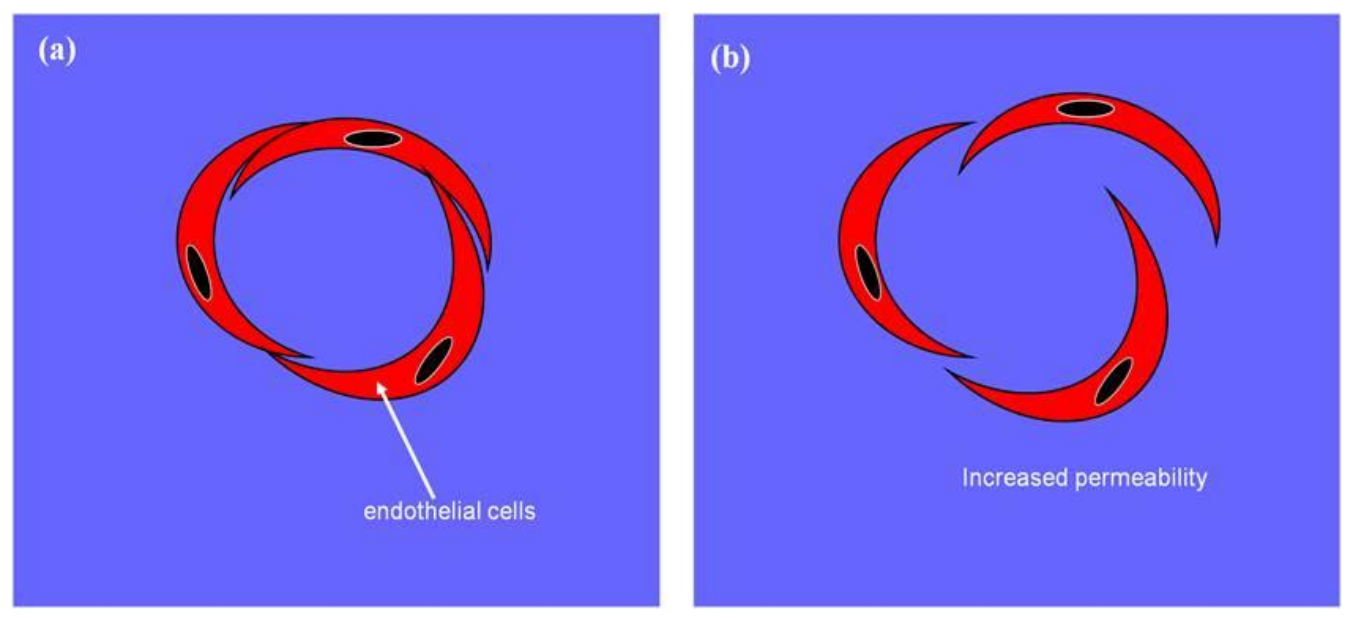

Figure 6-1. Permeability of blood capillaries during an inflammatory response.

Note: (a) represents blood capillaries before inflammation and (b) represents blood capillaries after inflammation. 
junctions of blood capillaries under normal and inflamed conditions.

Rubor is due to erythrocyte infiltration and pain is mainly the result of edema and kinins. Local edema may activate local deep pain receptors (throbbing pain, peaks with systolic pressure). Kinins act directly on nerve ends to produce pain sensation (pain of bee sting: the activation of a kinin in bee venom). The exact cause of heating during acute inflammation is not clear. However, there are numerous possible factors involved such as increased cellular metabolic activity, possible generation of pyrogens which are known to cause systemic fever and local disturbance of fluid flow.

\subsubsection{Chronic inflammatory response}

An inflammatory response that is persistent leads to chronic inflammation that is characterized by the presence of macrophages, monocytes and lymphocytes. It further involves proliferation of blood vessels and connective tissue. ${ }^{191,192,207,208}$ Chronic inflammation is usually less uniform histologically than acute inflammation and is dependent on chemical and physical properties of the biomaterial. The degree of chronic inflammation is also affected by motion in the implant site. Lymphocytes and plasma cells are involved principally in chronic immune reactions and are the key mediators of antibody production and delayed hypersensitivity responses. The macrophage produces great number of biologically active products such as neutral proteases, chemotactic factors, arachidonic acid metabolites, reactive oxygen metabolites, complement components, coagulation factors, growth-promoting factors, and cytokines. ${ }^{208}$ The growth factors in turn can further stimulate production of a wide variety of cells thereby initiating cell migration, differentiation and tissue remodeling and thus may be involved in various stages of wound healing.

\subsubsection{Granulation tissue}

Granulation tissue formation represents healing of inflammation and occurs within one day after implantation of biomaterial. The healing response is initiated by reaction of monocytes and macrophages and fibroblasts and vascular endothelial cells in the implant site proliferate and begin to form granulation tissue. There is a proliferation of new blood vessels (angiogenesis or neovascularization) and fibroblasts that can be seen as a pink, soft granular appearance of the granulation tissue on the surface of healing wounds.

\subsubsection{Foreign body giant cells (FBGCs)}

The foreign body reaction to biomaterials is composed of foreign-body giant cells (FBGCs) and components of granulation tissue (macrophages, fibroblasts, and capillaries). Fabric materials generally have a surface response composed of macrophages and foreign body giant cells, with varying degrees of granulation tissue 
subsequent to the surface response. The foreign-body reaction consists mainly of macrophages and/or foreign-body giant cells that may persist at the tissue-implant interface for the lifetime of the implant. Generally, fibrosis (i.e. fibrous encapsulation) surrounds the biomaterial with its foreign body reaction from the local tissue environment (Figure 6-2). Foreign body response depends on the form and geometry of the implant.

- Flat and smooth surfaces such as those found on breast implants; foreign body response is composed of a layer of macrophages one to two cells in thickness.

- Relatively rough surfaces such as those found on the outer surfaces of vascular prosthesis; foreign body response is composed of multiple layers of macrophages and foreign body giant cells at the surface.

- Rough surfaces such as fabric-type materials; composed of macrophages and foreign body giant cells with varying degrees of granulation tissue.

\subsubsection{Fibrosis/fibrous encapsulation}

Fibrosis/fibrous encapsulation is the final healing response to biomaterials and involves two distinct processes:

- Regeneration, which is replacement of injured tissue by parenchymal cells.

- Replacement by connective tissue.

These processes are controlled by either the proliferative capacity of the cells in the tissue or organ or persistence of the tissue framework of the implant sites. The regenerative capacity of cells can be classified into 3 groups i.e. labile, stable and permanent:

- Labile: cells continue to proliferate throughout life.

- Stable: cells retain capacity and do not replicate.

- Permanent: cells cannot reproduce themselves after birth.

Perfect repair with restitution of normal structure can theoretically occur only in tissues consisting of stable and labile cells, whereas all injured tissues composed of permanent cells may give rise to fibrosis and fibrous capsule formation with very little restitution of the normal tissue or organ structure. Figure 6-3 shows the temporal variation in various inflammatory responses. Histological examination of tissue adjacent to the formulation to determine intensity of inflammation, granulation, foreign body reaction and fibrosis, as a function of implant time, has been the most commonly used method to assess the biocompatibility of drug delivery systems or biomaterials. ${ }^{61}$ Individually both PLGA and polycaprolactone have shown appropriate biocompatibility. In rats, $\mathrm{d}, 1$ lactide/glycolide copolymer microspheres have been shown to be biocompatible for implantation with early mild inflammation, which resolved along with 


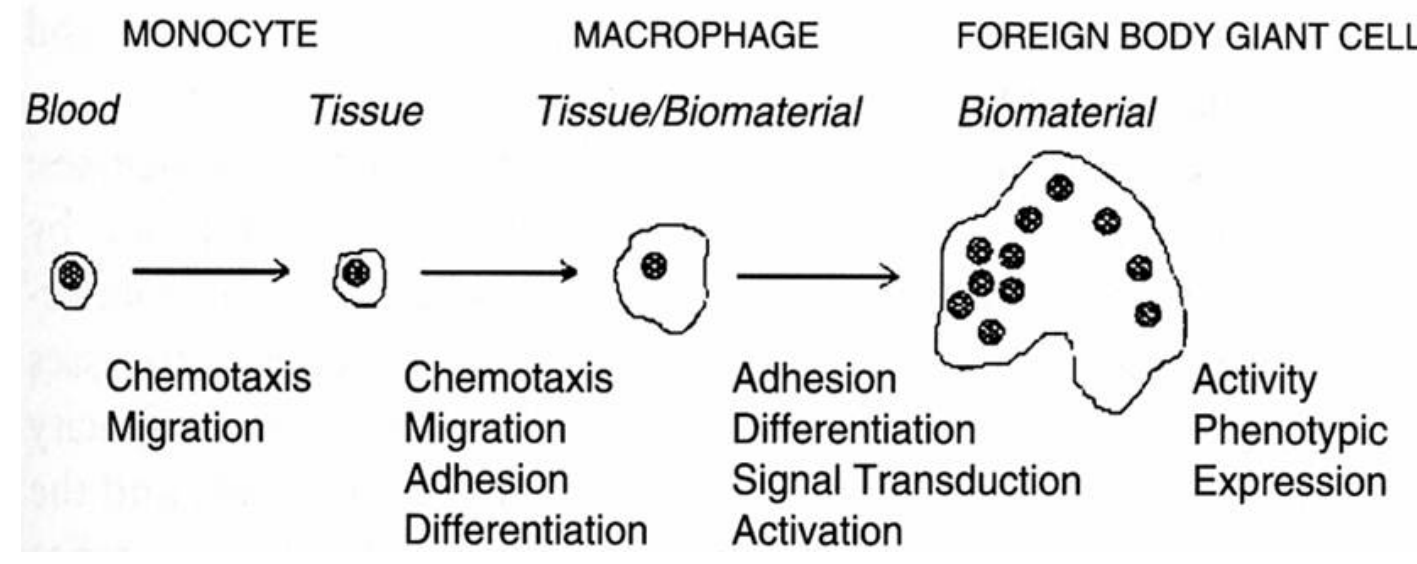

Figure 6-2. In vivo transition from blood-borne monocyte to biomaterial adherent monocyte/macrophage to foreign body giant cell at the tissue/biomaterial interface.

Note: Little is known regarding the indicated biological responses that are considered to play important roles in the transition to foreign body giant cell development. ${ }^{188}$

Source: Reprinted with permission from Elsevier. J. M. Biological responses to materials. Annu Rev Mater Res 31, 81-110 (2001). ${ }^{189}$ 


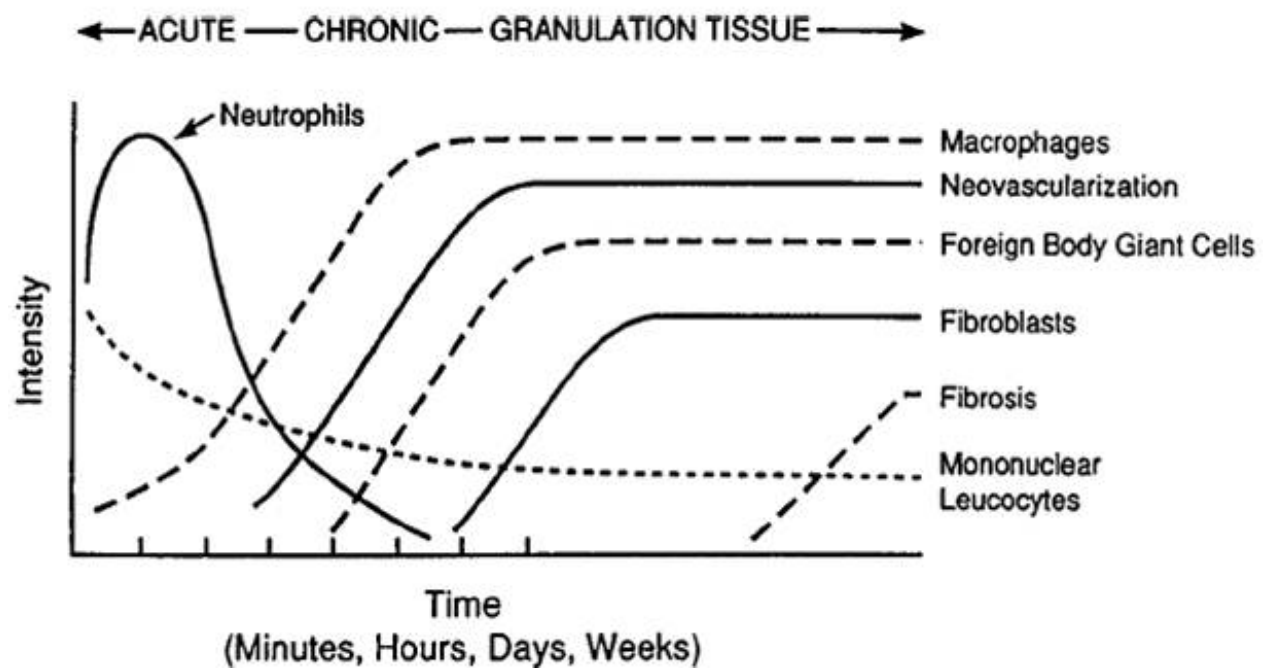

Figure 6-3. The temporal variation in the acute inflammatory response, chronic inflammatory response, granulation tissue development, and foreign body reaction to implanted biomaterials.

Note: The intensity and time variables are dependent upon the extent of injury created in the implantation and the size, shape, topography, and chemical and physical properties of the biomaterial. ${ }^{188}$

Source: Reprinted with permission from Elsevier. J. M. Biological responses to materials. Annu Rev Mater Res 31, 81-110 (2001). ${ }^{189}$ 
biodegradation, with no adverse tissue reaction, and the vast majority of microsphere degradation occurring by day $150 .{ }^{209}$ Biocompatibility of polycaprolactone films has been determined in mouse fibroblasts by Serrano et al. ${ }^{210}$ However, as mentioned earlier, the topography and surface characteristics are extremely important factors that determine the overall inflammatory response to biomaterial devices; it becomes necessary for us to evaluate theinflammatory response to the novel PLGA micro particulate polycaprolactone crystallite drug delivery system.

Since the in situ implant is fabricated as minute micron size crystallites or spherulites that are essentially a collection of sharp edged crystals, it presents greater likelihood of demonstrating an inflammatory response upon injection into the subcutaneous tissue. It was therefore the objective of this study to evaluate the inflammatory responses to the novel PLGA: PCL based implant system. Biodegradation is another parameter of in vivo response to the biomaterial devices. It is extremely important for the device material to be biodegradable on its own thereby eliminating the need for surgical removal of the devices or implants. The degradation kinetics of the biomaterial is studied during biodegradability determination. Numerous mechanisms could be involved in the biodegradation process depending on the type and characteristics of biomaterial and therefore all possible degradation mechanisms should be explored during the study. It is also the objective of this study to determine the biodegradability behavior of the novel PLGA: PCL based formulation.

\subsection{Materials and Methods}

\subsubsection{Materials}

PLGA (IV: $0.59 \mathrm{dL} / \mathrm{g}$ ) was purchased from Lactel absorbable polymers, Durect Corporation, Pelham, AL, USA. Glyceryl monooleate (Capmul GMO-50, EP/NF) was obtained as a generous gift from Abitec Corp., Janesville, WI, USA. PCL (polycaprolactone, MW 10,000) was obtained from The Dow Chemical Co., Midland, MI, USA. Leuprolide acetate (model drug) was purchased from Teva Pharmaceuticals, Israel. NMP (N-methyl pyrrolidone; Pharmasolve) was procured from ISP Pharma Technologies, Wayne, NJ, USA. ATEC (acetyl triethyl citrate) was obtained as a generous gift from Morflex Inc., Greensboro, NC, USA. All other reagents and chemicals were of pharmaceutical grade.

\subsubsection{Methods}

\subsubsection{Preparation of drug loaded PLGA micro particles}

Micro particles were prepared by a cryo film grinding method previously described in details in Chapter 2. Briefly, the PLGA polymer was dissolved in acetone 
followed by dispersing the required amount of drug into the polymer solution. The overall drug loading obtained was $16 \% \mathrm{w} / \mathrm{w}$ of polymer weight. The acetone was allowed to evaporate under controlled air flow conditions and the semi dried polymer mass thus obtained was transferred to a petri dish and kept in vacuum oven for 48 hours. The dried polymer film was then ground into micro particles by using a cryo mill.

\subsubsection{Determination of drug content in micro particles}

Drug content in the micro particles was determined by a liquid-liquid extraction procedure as described in Chapter 2. The drug content was analyzed by a RP-HPLC procedure.

\subsubsection{Preparation of injection vehicle containing PCL crystallites}

Injection vehicle was prepared as mentioned in Chapter 5. Briefly, 1 part by weight of NMP was mixed with 9 parts by weight of ATEC. $8 \% \mathrm{w} / \mathrm{w}$ of PCL was added to the above mixture and allowed to melt completely under controlled heating. GMO (glyceryl monooleate) was added to above solution in a weight ratio of 1:1 and the entire system was allowed to cool gradually under normal room temperature conditions. This resulted in formation of PCL crystallites with a uniform geometry.

\subsubsection{Injection of the in situ implant formulation}

A male Sprague-Dawley rat model was selected for the in vivo biodegradability and biocompatibility studies. The formulation components i.e. drug loaded PLGA micro particles and the PCL crystallite containing injection vehicle were mixed intimately with each other using a two syringe system with male and female leur locks and injected into the subcutaneous tissue in the left flank region of the rats. The injections were made using a 20 gauge needle and the injection site was held between fingers for a few seconds to prevent the liquid formulation from leaking out. The animals were caged in a group of 3 with solid bottom caging. A dose equivalent of $7.5 \mathrm{mg} / \mathrm{Kg}$ of animal weight of leuprolide acetate was injected into the animals. At 7, 14, 30 and 48 days, one rat was sacrificed by using an overdose euthasate. The injection site was incised and the diameter of the implant at injection site was measured. The tissue surrounding implant was removed and immediately transferred to a $10 \%$ buffered formalin solution for further histological evaluation.

\subsubsection{Preliminary histological preparation}

The collected biopsy tissue samples were fixed in $10 \%$ buffered formalin and then submitted for routine histological processing. This involved saturation of tissue with organic solvents (i.e. alcohol dilutions, then xylene). The biopsy specimens were then 
infiltrated with heated paraffin and set in paraffin blocks. Sections were cut from the blocks at $5 \mu \mathrm{m}$ in thickness, placed on a slide, stained with haematoxylin and eosin and covered by a glass cover-slip. 4 slides were prepared per tissue sample for microscopic analysis and scored according to the following system: $0=$ no infiltration up to $10=$ severe infiltration of leukocytes, lymphocytes, and macrophages.

\subsection{Results and Discussions}

\subsubsection{Evaluation of biodegradability of implant}

Figure 6-4 shows the images for implant remaining at the injection site at 7, 14, 30 and 42 days. The implant was identified as a semi-solid or solid gel in the subcutaneous tissue at day 7 post injection. A distinct layer of tissue can be seen around the implant indicating the encapsulation process. The in vivo degradation profile (Figure 6-5) shows a gradual reduction in the implant size over time. This is significantly different from the normal PLGA degradation process, whereby a very slow degradation phenomenon occurs for the first 20 to 30 days after which considerable polymer degradation happens within next few days attributed to the final bulk erosion process. A more gradual degradation process essentially indicates a more diffusion controlled drug release process with the novel PLGA micro particles: PCL crystallite formulation compared to the PLGA alone based formulations. PCL crystallite network allow a gradual release of the plasticizer from the formulation into the surrounding tissue thereby resulting in sufficient plasticization of the PLGA micro particles over time. The plasticized PLGA polymer then releases the drug in mainly a diffusion controlled manner than erosion dominated process.

\subsubsection{Biocompatibility of implant}

The primary concern in this study was the local tissue responses instead of systemic toxicity to the in situ implant formulations. Both the polymer and solvent used in drug delivery systems play an important role in the toxicity to the body. ${ }^{182}$ Therefore, we first evaluated the biocompatibility of the injection vehicle comprised of the PLGA polymer plasticizers i.e. NMP and ATEC, and the glycerol lipid (GMO). The skin tissue containing subcutis and muscle were excised at day 30 from the site of injection and subjected to histological examination. The tissue samples showed no evidence of an inflammatory cellular response. The subcutis revealed only a small lymph node in one of the tissue samples apparently associated with the mammary chain and there was no evidence of any lymphoid infiltrate (Figure 6-6).

The inflammatory response from the test formulation consisting of drug loaded PLGA micro particles dispersed uniformly in the injection vehicle constituted of polymer plasticizers (ATEC and NMP), GMO and PCL crystallite was evaluated after 7, 14, 30 and 48 days of subcutaneous injection. Figure 6-7 shows the tissue response after 7 days 

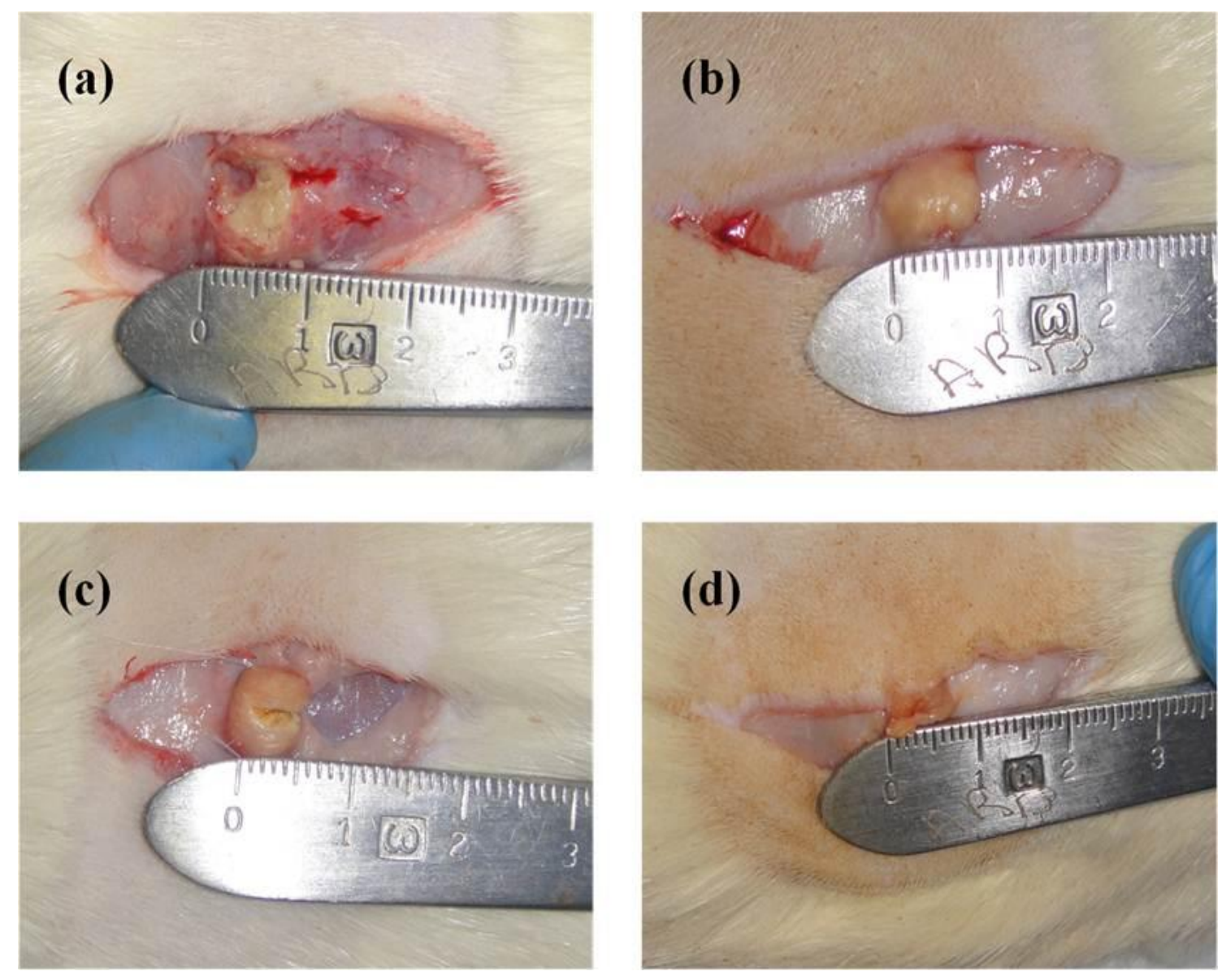

Figure 6-4. Implant remaining at the site of injection at (a) day 7; (b) day 14; (c) day 30; and (d) day 48 post injection. 


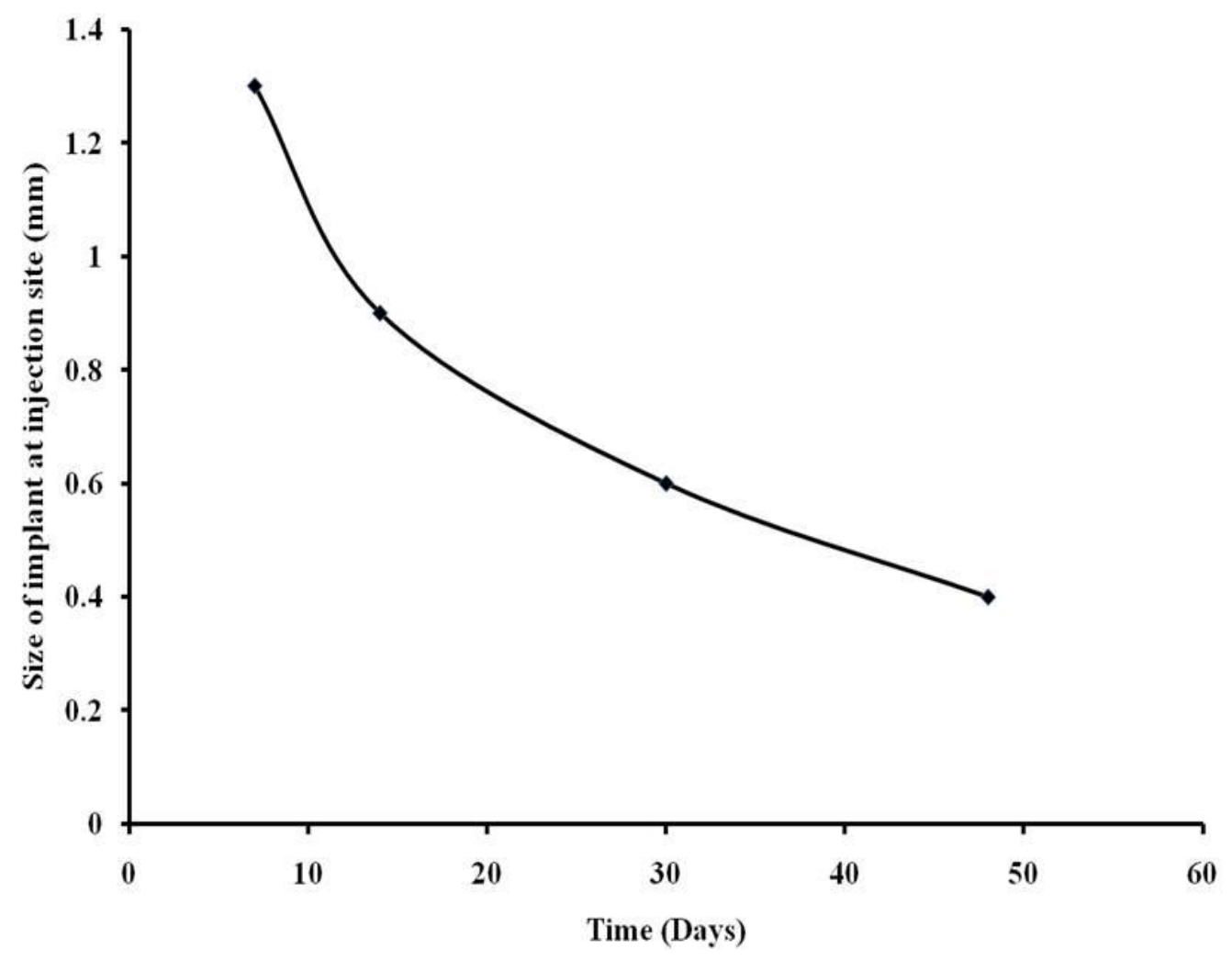

Figure 6-5. Biodegradation profile for the in situ PLGA micro particles: PCL crystallite implant.

Note: Each data point represents the measured implant diameter remaining at the site of injection. 

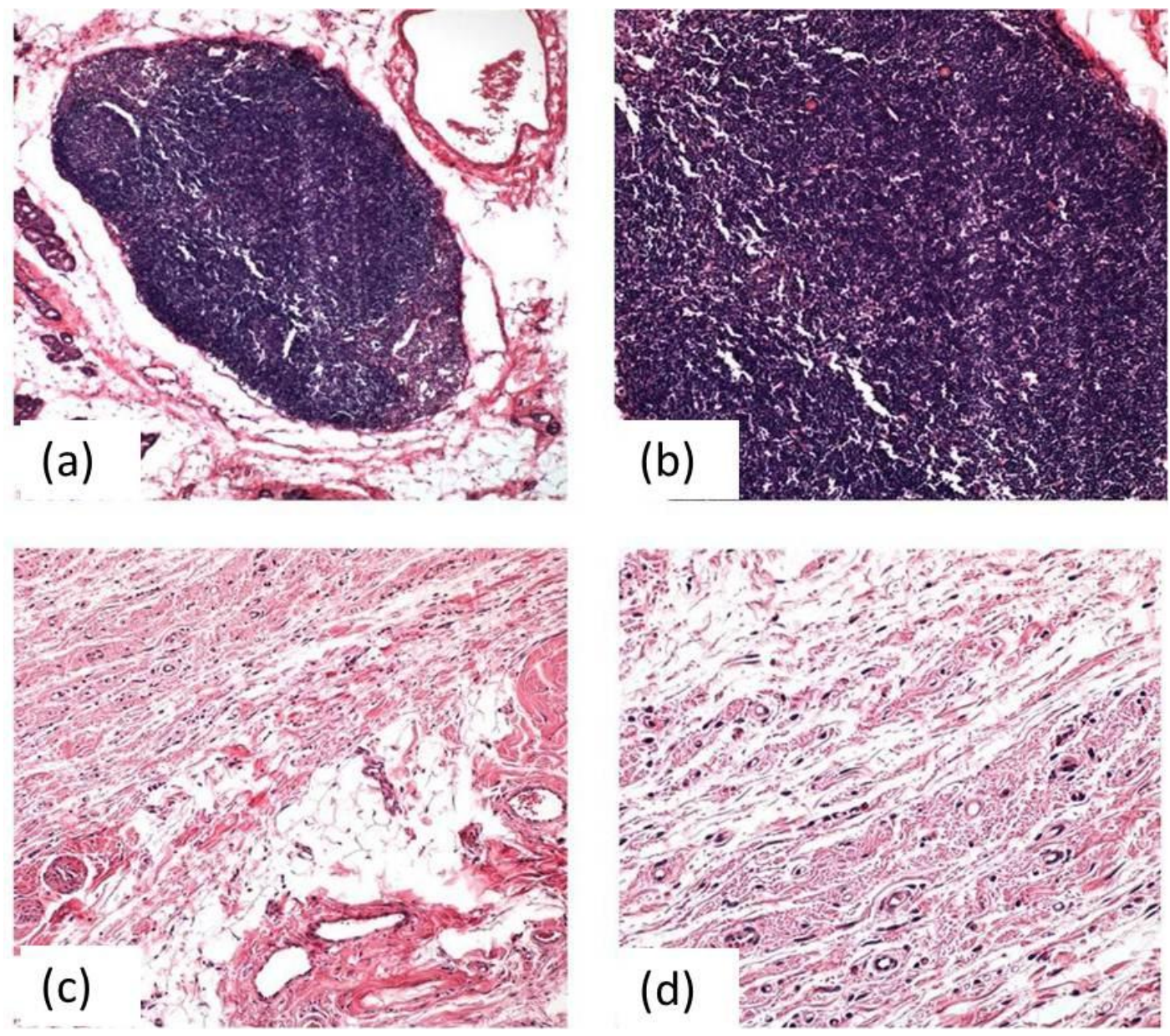

Figure 6-6. Light photomicrograph of subcutaneous tissue 30 days after subcutaneous injection of test formulation consisting of injection vehicle solvent at (a) 10x; (b) and (c) 20x; and (d) 40x magnification.

Notes: Photomicrographs (a) and (b) represent one animal and (c) and (d) represent the second animal sacrificed at day 30 post injection.

The subcutis revealed only a small lymph node in the first animal with no evidence of lymphoid infiltrate.

The subcutis had an essentially normal appearance with no evidence of lymphoid cells in second animal. 

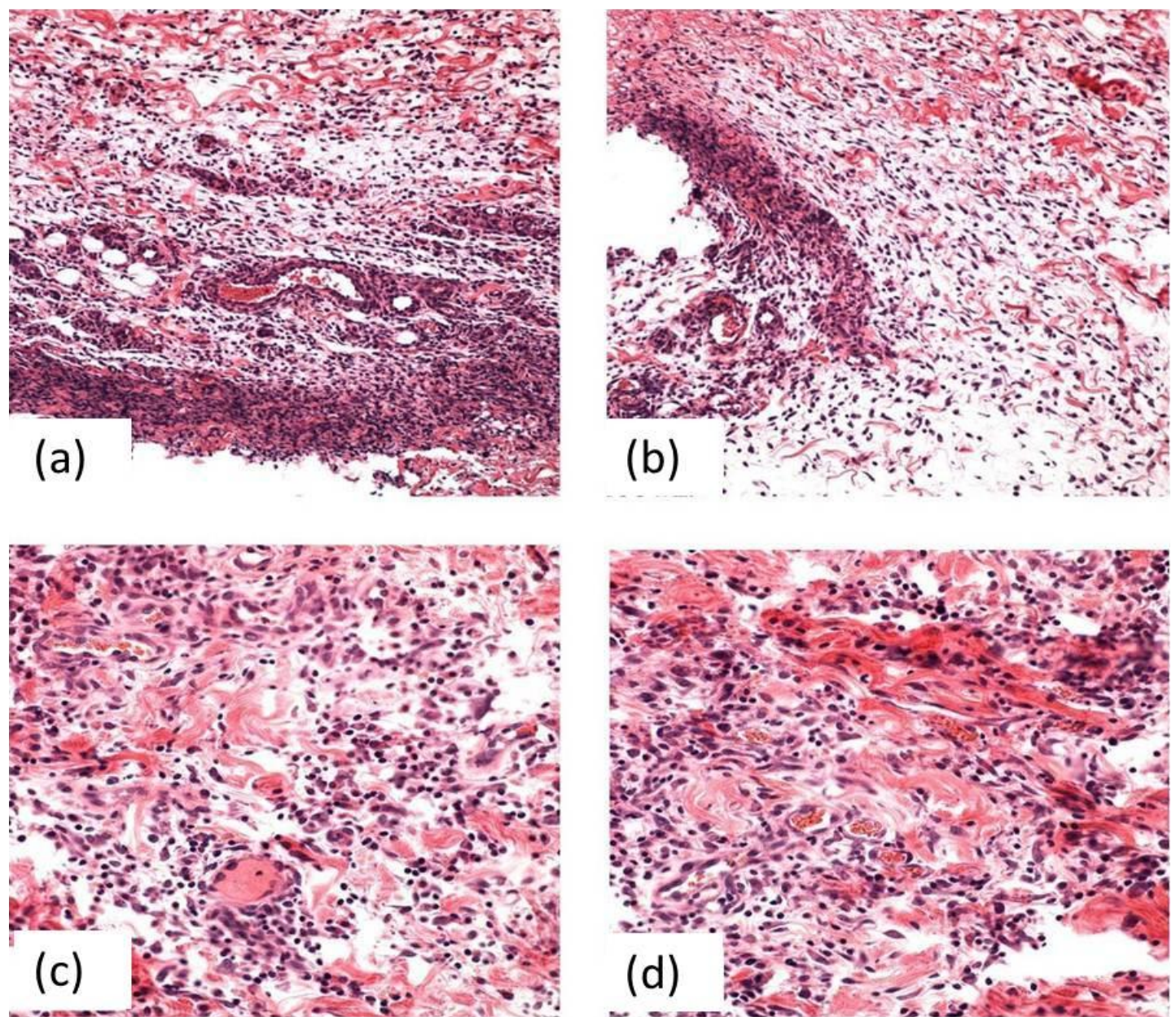

Figure 6-7. Light photomicrograph of subcutaneous tissue 7 days after subcutaneous injection of test formulation consisting of drug loaded PLGA micro particles dispersed in a PCL crystallite based injection vehicle at (a) and (b) 20x; and (c) and (d) 40x magnification.

Notes: Photomicrographs (a) and (b) represent one animal and (c) and (d) represent the second animal sacrificed at day 7 post injection.

A prominent influx of mostly lymphoplasmacytic cells was present between the subcutis for the first animal. Evidence of fibrovascular proliferation was mild.

A prominent influx of mixed chronic inflammatory cell infiltrate was present within the subcutis and consisted primarily of lymphocytic cells and a moderate fibrovascular (granulation) tissue deposition. 
of subcutaneous injection of the in situ forming implant formulation. A prominent influx of mostly lymphoplasmacytic cells was present within the subcutis along with the evidence of a mild fibrovascular proliferation. Another sample at day 7 showed a prominent influx of mixed chronic inflammatory cell infiltrate within the subcutis and consisted primarily of lymphocytic cells and a moderate fibrovascular (granulation) tissue diposition. Overall, the cellular response was mostly lymphoplasmacytic with mild to moderate evidence of fibrosis (fibrovascular response).

At day 14, the subcutis revealed a focus of essentially mature lymphoid cells that might have been associated with the adnexal structures. Further, there was only a minimal evidence of fibrosis (Figure 6-8). The subcutis of a replicate tissue sample showed essentially no evidence of a lymphocytic infiltrate (data not shown). The average tissue response compared to that observed after 7 days, was a much reduced inflammation.

Figure 6-9 shows the tissue response after 30 days of subcutaneous implantation of the formulation. The response ranged from several small foci of essentially mature lymphoid cells to an almost of no evidence of a lymphoid infiltrate. Further, there was no evidence of fibrosis in any of the tissue samples collected at day 30. After 48 days, the subcutis had an essentially normal appearance with no evidence of lymphoid cells (Figure 6-10).

Further, a grading scale of 0 to +10 was used to compare the inflammatory response from the test formulations and the positive control (Figure 6-11). The absence of an inflammatory infiltrate was given a grade of 0 , the smallest cellular infiltrates were given a grade of +1 and the most prominent lesions were given a score of +10 . The animals that received test formulation with injection vehicle solvents had no evidence of an inflammatory cellular response with an average score of 0 .

The animals receiving test formulation with PLGA polymer micro particles dispersed in injection vehicle and removed on day 7 from the study also had an average score of +10 . The cellular response was mostly lymphoplasmacytic with mild to moderate evidence of fibrosis. The animals removed on day 14 had only an average score of +2 with a range of 0 to +4 and those removed on day 30 had an average score of +1.25 with a range of 0 to +2 . Finally, the animals removed on day 48 had an average score of +0.5 with a range of 0 to +1 .

The objective of this study was essentially to evaluate and determine the inflammatory potential of polymeric crystallites or spherulites upon injection into the subcutaneous tissue. Studies have indicated that inflammatory response is directly associated with particle size, shape and irregularity. ${ }^{211,212}$ Sharp edge particles tend to induce greater inflammatory response. ${ }^{213}$ SEM studies for PCL crystallites (Chapter 5) have demonstrated a highly irregular surface but essentially no sharp edges. It was therefore essential for us to understand the possible tissue reaction of such crystallites. The biocompatibility studies, however, have indicated that the in situ implant formulation containing PCL crystallites is safe and biocompatible with only a normal tissue response. 

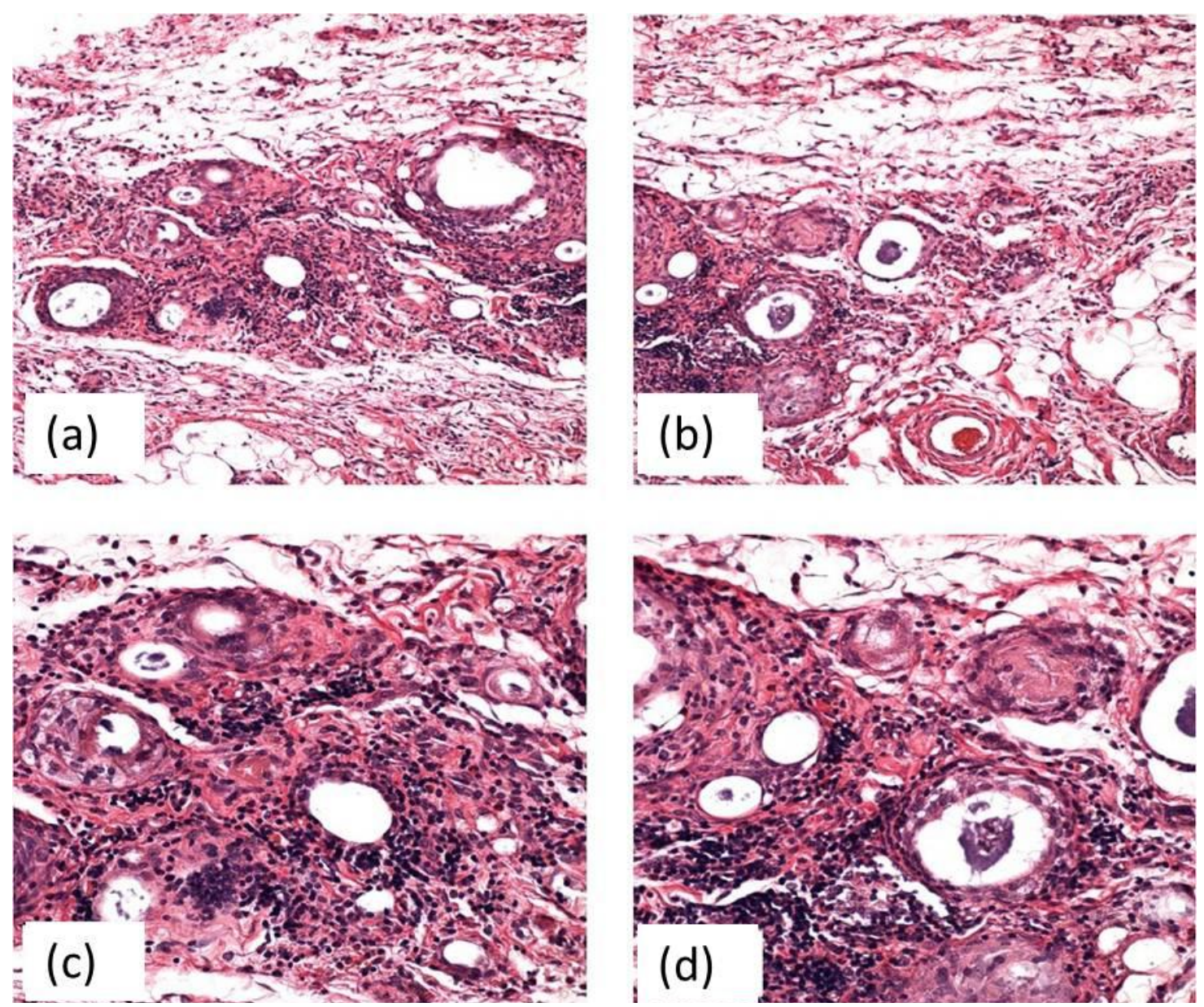

Figure 6-8. Light photomicrograph of subcutaneous tissue 14 days after subcutaneous injection of test formulation consisting of drug loaded PLGA micro particles dispersed in a PCL crystallite based injection vehicle at (a) and (b) 20x; and (c) and (d) 40x magnification.

Note: The subcutis revealed a focus of essentially mature lymphoid cells and evidence of fibrosis was minimal. 

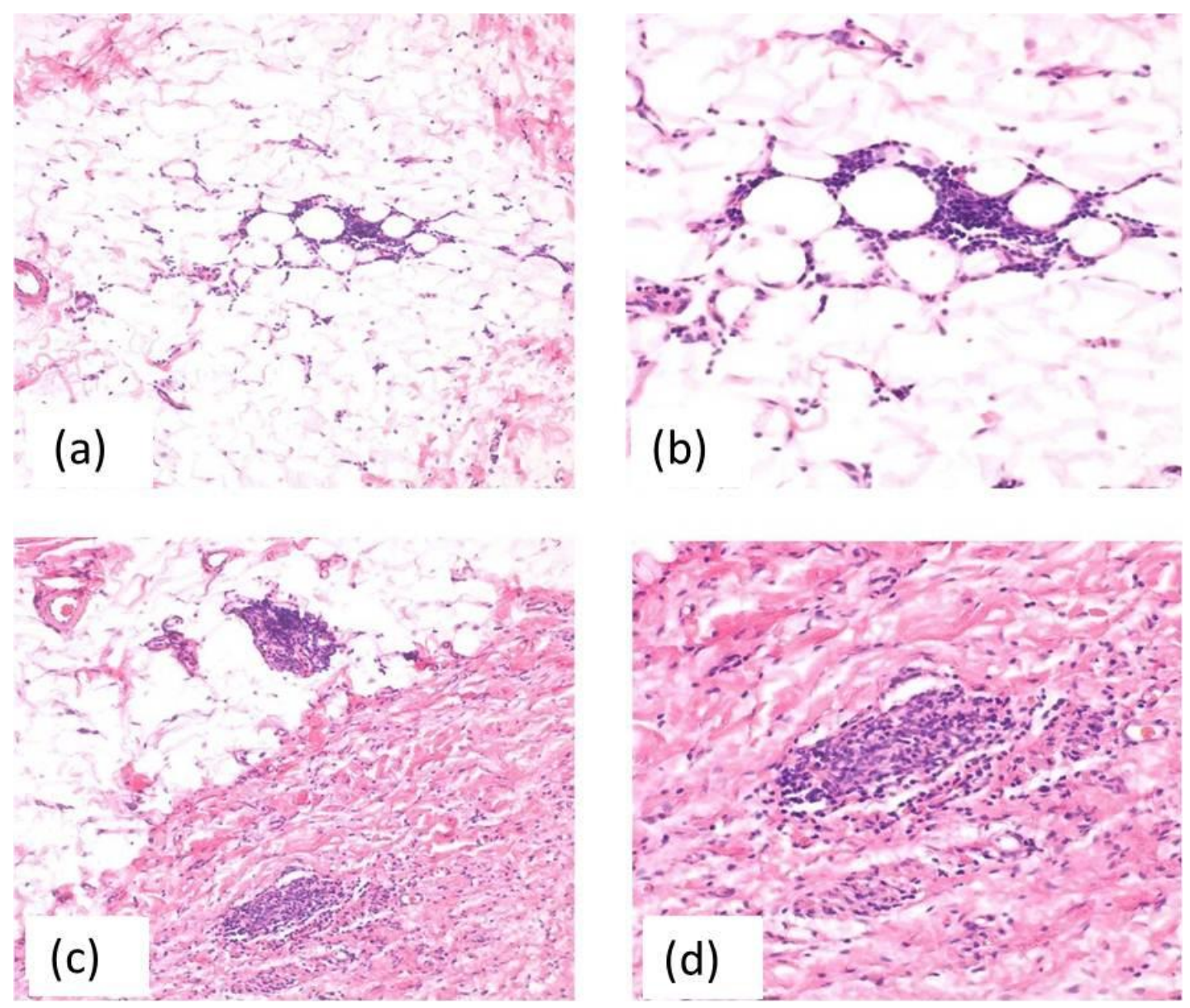

Figure 6-9. Light photomicrograph of subcutaneous tissue 30 days after subcutaneous injection of test formulation consisting of drug loaded PLGA micro particles dispersed in a PCL crystallite based injection vehicle at (a) and (c) 20x; and (b) and (d) 40x magnification.

Notes: Photomicrographs (a) and (b) represent one animal and (c) and (d) represent the second animal sacrificed at day 30 post injection.

The subcutis revealed one very small focal influx of mostly mature lymphocytes. There was no definitive evidence of fibrosis for the first animal.

The subcutis revealed several small foci of essentially mature lymphoid cells and there was no definitive evidence of fibrosis for the second animal. 

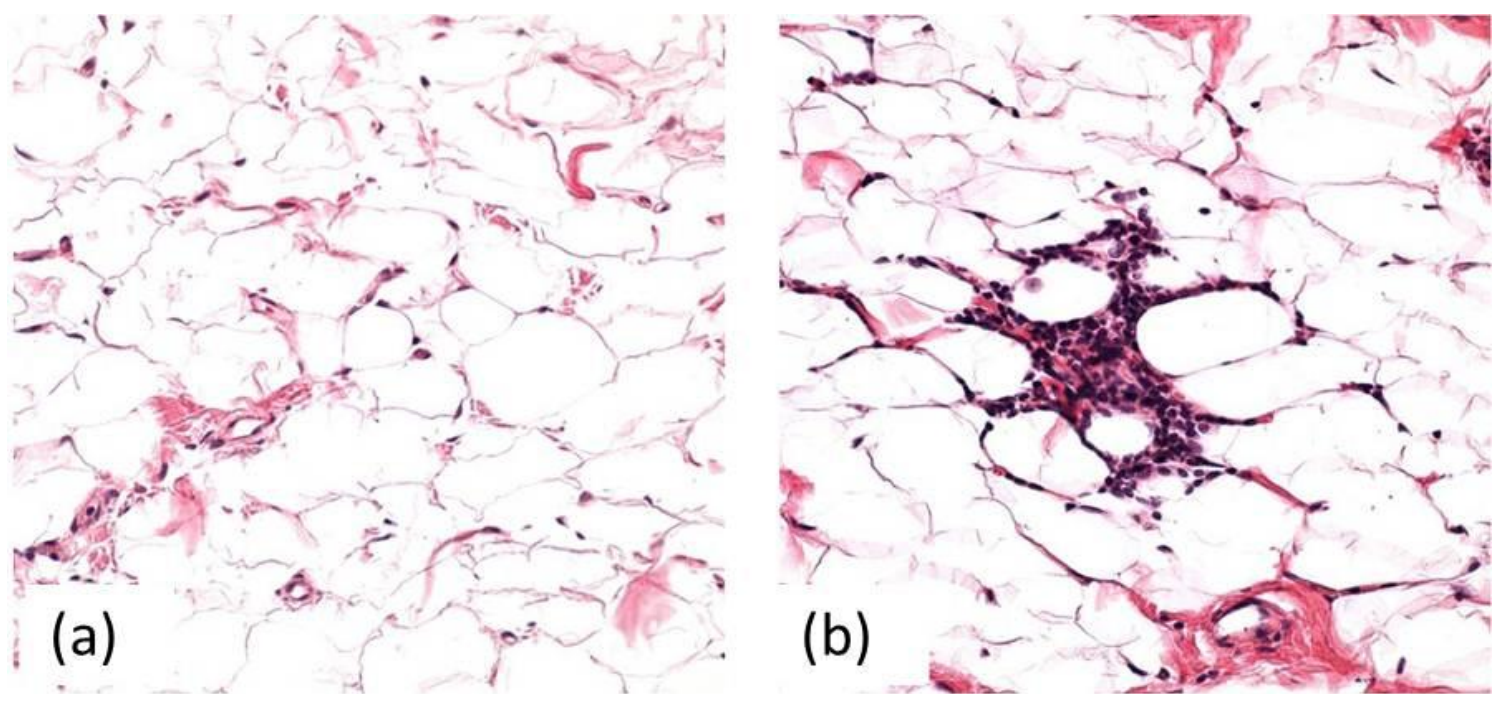

Figure 6-10. Light photomicrograph of subcutaneous tissue 48 days after subcutaneous injection of test formulation consisting of drug loaded PLGA micro particles dispersed in a PCL crystallite based injection vehicle at $40 \mathrm{x}$ magnification.

Notes: Photomicrograph (a) represents one animal and (b) represents the second animal sacrificed at day 48 post injection.

The subcutis had an essentially normal appearance with no evidence of lymphoid cells for the first animal.

The subcutis revealed only a very small focus of mostly mature lymphoid cells. 


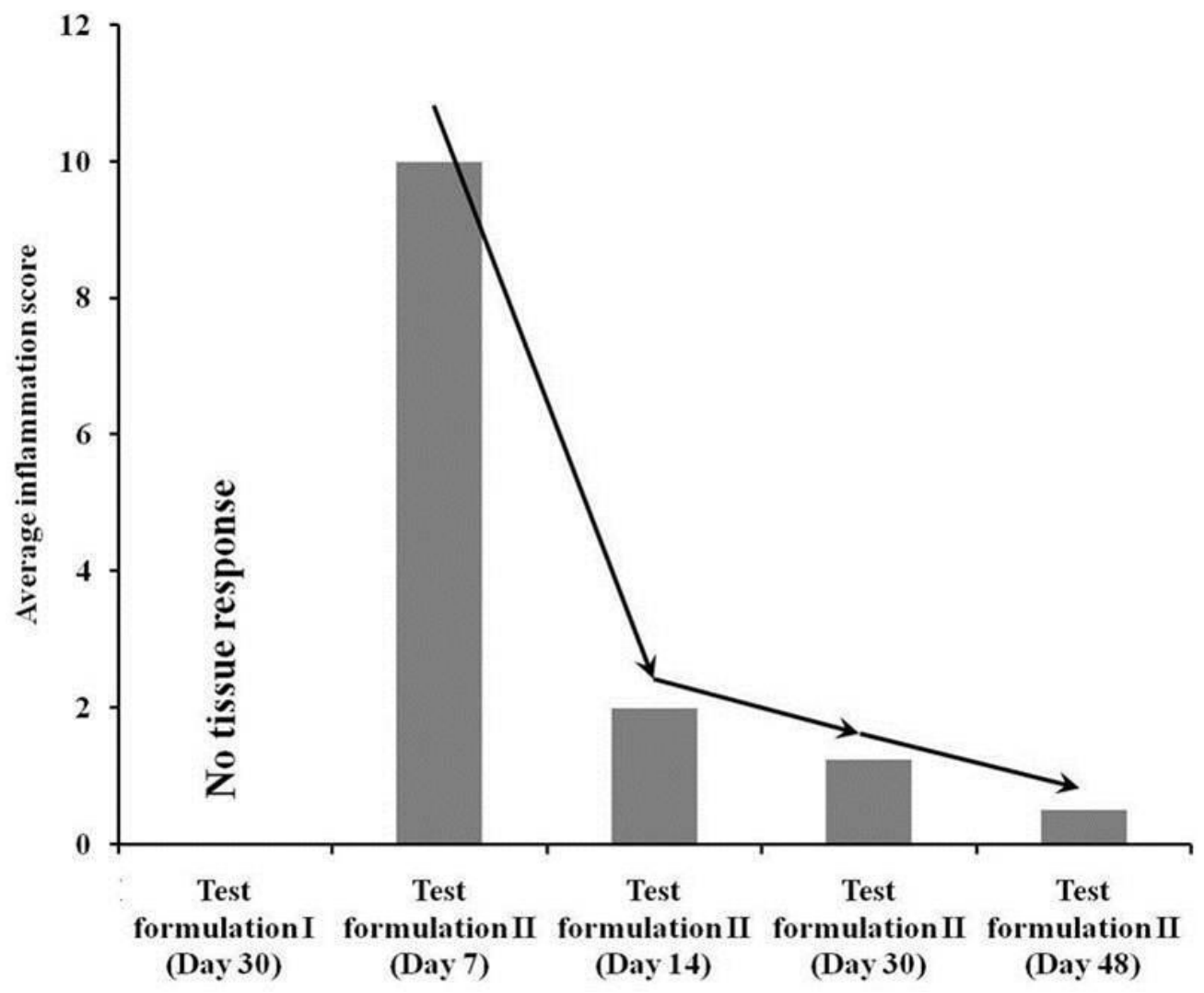

Figure 6-11. Average score for the inflammatory tissue response for control and test formulations.

Note: Test formulation I represents the injection vehicle solvent and test formulation II represents the novel PLGA micro particulate- PCL crystallite formulation. 


\subsection{Conclusion}

The in vivo biodegradability and biocompatibility of the novel PLGA micro particulate- PCL crystallite implant formulation was investigated in this study. The results showed that the in situ implant had good biodegradability with a significant reduction in implant size by the end of 48 days. Moreover, the degradation process was very gradual with a continuous reduction in implant size unlike most of the PLGA alone based in situ implant systems that degrade by a bulk erosion phenomenon. Histological examination of the tissue from the site of injection showed an initial moderate tissue response with a significant reduction in tissue response by the end of 14 days. The inflammatory response was almost completely subsided at the end of 48 days post injection. The animals administered with the test formulation containing injection solvent only showed no evidence of cellular infiltrate. Overall, the biocompatibility studies demonstrated a completely normal tissue response from the PCL crystallite based test formulation. 


\section{RESEARCH SUMMARY}

A novel in situ gelling polymer depot system has been designed for controlled delivery of drugs which is based on a drug loaded PLGA micro particulate suspension formulation. The formulation was given desired rheological properties such as shear thinning and thixotropic characteristics for the ease of administration. The necessary rheological properties were introduced by a novel carrier or vehicle intended for reconstitution of the drug loaded micro particles. The vehicle was constituted of three key components:

- Hydrophobic solvent(s)

- Polymer immiscible component

- Polymeric crystallites/spherulites

An optimized solvent combination of ATEC and NMP (9:1 w/w) was used as the hydrophobic solvent. The hydrophobic solvent due to its limited affinity for the external aqueous environment stayed in contact with the PLGA polymer matrix for a sufficient length of time to be able to provide a continuous drug release based on solvent diffusion. The micro particulate suspension formulation overall resulted in a significantly lower viscosity than a polymer solution made with the same hydrophobic solvent.

Since the system was formulated as a micro particulate suspension, it was important to introduce the desired rheological characteristics in the formulation to make it structurally more stable. Structural stability in polymer micro particulate suspensions means that the interaction between the polymer solvent in the vehicle and the micro particles is limited to a sufficient extent in order to have less inter individual variability in the properties of the reconstituted suspension for better injectability and ease of administration. The objective was successfully accomplished with two key components of the injection vehicle, the glycerol lipid and the polymeric crystallites/spherulites.

The polymer immiscible component was a glycerol lipid (glyceryl monooleate), which due to immiscibility with PLGA polymer was able to act as an anti-solvent and limit the overall interaction between the PLGA micro particles and the hydrophobic solvent. A ternary phase diagram was constructed with the three components being the polymer (PLGA), the glycerol lipid (GMO) and the optimized hydrophobic solvent (ATEC:NMP 9:1 w/w) in order to identify two distinct phases, the solution phase and the stable suspension phase. The vehicle composition selected from the suspension phase demonstrated greater structural stability of the micro particulate suspension formulation than the solution phase. Further, it was possible to modulate the drug release rate with the help of different glycerol lipid based vehicle compositions. Mechanistic studies were conducted by comparing percent drug released with percent solvent released during the course of study and results indicated overall drug release behavior changing from mainly polymer erosion controlled to primarily diffusion controlled with increasing percentage of the hydrophobic solvent. Further comparing micro environmental $\mathrm{pH}$ with the $\mathrm{pH}$ of 
bulk dissolution media indicated a greater diffusion of the PLGA acidic degradation products at higher levels of hydrophobic solvent in the formulation. This essentially confirmed the overall diffusion controlled drug release at higher solvent levels.

The polymeric crystallites were designed with the polycaprolactone polymer. Polycaprolactone exists as white waxy pellets and is a semi crystalline polymer but once co-heated with the other vehicle components i.e. the hydrophobic solvent and glycero lipid followed by gradual cooling at room temperature, forms crystallites. These crystallites existed in clusters, which were hypothesized to be responsible for the desired thixotropic characteristic of the suspension based formulation. DSC studies demonstrated a partial plasticization of polycaprolactone polymer in the crystallites and SEM imaging showed a rough surface with numerous three dimensional projections for the crystallites. The crystallites essentially aggregated upon contacting the dissolution media thereby limiting the initial burst release of the drug. The crystallite based formulations exhibited a hysteresis loop in the viscosity measurements indicating their thixotropic behavior. This thixotropic characteristic is extremely crucial for the injectable suspension formulations as it keeps the PLGA micro particles well separated in the syringe prior to administration thereby resulting in lower injection force and good injectability. Mechanistic studies indicated drug diffusion mainly controlled by solvent diffusion from the novel formulation.

In vivo biocompatibility studies were conducted whereby the optimized PLGA micro particulate:PCL crystallite based formulation was injected into the subcutaneous tissue of male Sprague Dawley rats. The subcutis tissue from the site of injection was examined histologically for tissue reaction. Results indicated an initial tissue response similar to chronic inflammatory response at the end of first 7 days following which there was a significant reduction observed in the inflammatory response. The average inflammatory score reduced from +10 (maximum) at day 7 to less than +2 at day 14 thereby demonstrating well tolerability and biocompatibility of the novel formulation. 


\section{LIST OF REFERENCES}

1. Collins-Gold, L., Lyons, R. \& Batholow, L. Parenteral emulsions for drug delivery. Adv Drug Del Rev 5, 189-208 (1990).

2. Davis, S. \& Illum, L. Colloidal drug delivery systems: opportunities and challenges. (Wiley, 1986).

3. Florence, A. T. \& Whitehill, D. The formulation and stability of multiple emulsions. Int J Pharm 11, 277-308 (1982).

4. Allen, T. M., Hansen, C. B. \& Guo, L. S. Subcutaneous administration of liposomes: a comparison with the intravenous and intraperitoneal routes of injection. Biochim Biophys Acta 1150, 9-16 (1993).

5. Anderson, P. M., Katsanis, S. E., Sencer, S. F., Hasz, D. \& Bostrom, B. Depot characteristics and biodistribution of interleukin 2 liposomes: importance of route of administration. J. Immunother. 12, 19-31 (1990).

6. Bonetti, A., Chatelut, E. \& Kim, S. An extended release formulation of methotrexate for subcutaneous administration. Cancer Chemother Pharmacol 33, 303-306 (1994).

7. Sharma, A. \& Sharma, U. S. Liposomes in drug delivery: progress and limitations. Int J Pharm 154, 123-140 (1997).

8. Shenderova, A., Burke, T. G. \& Schwendeman, S. P. Stabilization of 10-hydroxy camptothecin in poly (lactide-co-glycolide) microsphere delivery vehicles. Pharm Res 14, 1406-1414 (1997).

9. Chen, L., R.N. Apte, R. N. \& Cohen, S. Characterization of PLGA microspheres for the controlled delivery of IL I for tumor immunotherapy. J Control Release 43, 261-272 (1997).

10. Cleland, J. L. \& Jones, A. J. Stable formulations of recombinant human growth hormone and interferon gamma for microencapsulation in biodegradable microspheres. Pharm Res 13, 1464-1475 (1996).

11. Zhang, X., Jackson, J. K. \& Bert, H. M. Development of amphiphilic diblock copolymers as micellar carriers of taxol. Int J Pharm 132, 195-206 (1996).

12. Alkan-Onyuksel, H., Ramakrishnan, S., Chai, H. B. \& Pezzuto, J. M. A mixed micellar formulation suitable for the parenteral administration of taxol. Pharm Res 11, 206-212 (1994).

13. Yim, Z., Zupon, M. A. \& Chaudry, I. A. Stable oleaginous gel. U.S. patent 4851220 (1989).

14. Woudenberg, I. A. J. M., Kate, M. T., Storm, G. \& van Etten, E. W. M. Administration of liposomal agents and the phagocytic function of the mononuclear phagocyte system. Int J Pharm 162, 5-10 (1998).

15. Moghimi, S. M. Opsono recognition of liposomes by tissue macrophages. Int $J$ Pharm 11-18 (1998).

16. Allen, C., Eisenberg, A. \& Maysinger, D. Copolymer drug carriers: conjugates, micelles and microspheres. Stp Pharma Sci. 9, 139-151 (1999).

17. Florence, A. T. \& Atwood, D. Physicochemical principles of pharmacy. (Macmillan Press, 1998). 
18. Lambert, W. J. \& Peck, K. D. Development of an in situ forming biodegradable poly-lactide-co-glycolide system for the controlled release of proteins. J Control Release 33, 189-195 (1995).

19. Gombotz, W. R. \& Pettit, D. K. Biodegradable polymers for protein and peptide drug delivery. Bioconjug Chem 6, 332-351 (1995).

20. Wood, D. A. Biodegradable drug delivery systems. Int J Pharm 7, 1-18 (1980).

21. Bezwada, R. S. \& Rao, S. Liquid copolymers of epsilon-caprolactone and lactide. U.S. patent 5442033 (1995).

22. Bezwada, R. S. \& Arnold, S. C. Liquid absorbable copolymers for parenteral applications. U.S. patent 5653992 (1997).

23. Einmahl, S. et al. A viscous bioerodible poly (ortho ester) as a new biomaterial for intraocular application. J Biomed Mat Res 50, 566-573 (2000).

24. Jackson, J. K. et al. A polymer based drug delivery system for the antineoplastic agent bis (maltolato) oxovanadium in mice. Br J Cancer 75, 1014-1020 (1997).

25. Holland, S. J., Tighe, B. J. \& Gould, P. L. Polymers for biodegradable medical devices: the potential of polyesters as controlled macromolecular release systems. J Control Release 4, 155-180 (1986).

26. Deshpande, A. A., Heller, J. \& Gurny, R. Bioerodible polymers for ocular drug delivery. Crit Rev Ther Drug Carrier Syst 15, 381-420 (1998).

27. Dunn, R. L., English, J. P., Cowsar, D. R. \& Vanderbilt, D. P. Biodegradable in situ forming implants and methods of producing the same. U.S. patent 4938763 (1990).

28. Dunn, R. L., English, J. P., Cowsar, D. R. \& Vanderbelt, D. D. Biodegradable in situ forming implants and methods of producing the same. U.S. patent 5278202 (1994).

29. Peter, S. J. et al. In vitro degradation of a poly (propylene fumarate) /b-tricalcium phosphate composite orthopedic scaffold. Tissue Eng 3, 207-215 (1997).

30. Burkoth, A. K. \& Anseth, K. S. A review of photocrosslinked polyanhydrides: in situ forming degradable networks. Biomaterials 21, 2395-2404 (2000).

31. West, J., L. \& Hubbell, J. A. Localized intravascular protein delivery from photopolymerized hydrogels. Proc Int Symp Control Rel Bioact Mat 22, 17-18 (1995).

32. Cui, H. \& Messermith, P. B. Thermally triggered gelation of alginate for controlled release. (American Chemical Society, 1998).

33. Dunn, R. L., Tipton, A. J., Southard, G. L. \& Rogers, J. A. Biodegradable polymer composition. U.S. patent 5599552 (1997).

34. Thote, A. J., Chappell, J. T., Jr., Gupta, R. B. \& Kumar, R. Reduction in the initial burst release by surface crosslinking of PLGA microparticles containing hydrophilic or hydrophobic drugs. Drug Dev Ind Pharm 31, 43-57 (2005).

35. Stile, R. A., Burghardt, W. R. \& Healy, K. E. Synthesis and characterization of injectable poly (N-isopropylacrylamide) based hydrogels that support tissue formation in vitro. Macromolecules 32, 7370-7379 (1999).

36. Bae, Y. H., Okano, T., Hsu, R. \& Kim, S. W. Thermosensitive polymers as on/off switches for drug release. Makromol Chem Rapid Commun 8, 481-485 (1987). 
37. Hoffman, A. S. Applications of thermally reversible poly mers and hydrogels in therapeutics and diagnostics. J Control Release 6, 297-305 (1987).

38. Ericsson, B., Ericsson, P. O., Lofroth, J. E. \& Engstrom, S. Cubic phases as drug delivery systems for peptide drugs. ACS Symp Ser 469, 251-265 (1991).

39. Gao, Z., Crowley, W. R., Shukla, A. J., Johnson, J. T. \& Reger, J. F. Controlled release of contraceptive steroids from biodegradable and injectable gel formulations: in vivo evaluation. Pharm Res 12, 864-868 (1998).

40. Gao, Z., Shukla, A. J., Johnson, J. R. \& Crowley, W. R. Controlled release of contraceptive steroid from biodegradable and injectable gel formulations: in vitro evaluation. Pharm Res 12, 857-864 (1995).

41. Appel, L., Engle, K., Jensen, J., Rajewski, L. \& Zentner, G. An in vitro model to mimic in vivo subcutaneous monoolein degradation. Pharm Res 11, 217 (1994).

42. Hatefi, A. \& Amsden, B. Biodegradable injectable in situ forming drug delivery systems. J Control Release 80, 2-28 (2002).

43. Alzoum, M. A., Alorainy, I. A., Al Husain, M. \& Al Ruhaimi, K. Multiple pericallosal lipomas in two siblings with frontonasal dysplasia. AJNR Am J Neuroradiol 23, 730-731 (2002).

44. Gopferich. Polymer bulk erosion. Macromolecules 30, 2598-2604 (1997).

45. Shive, M. S. \& Anderson, J. M. Biodegradation and biocompatibility of PLA and PLGA microspheres. Adv Drug Del Rev 28, 5-24 (1997).

46. Jain, R. A. The manufacturing techniques of various drug loaded biodegradable poly (lactide-co-glycolide) (PLGA) devices. Biomaterials 21, 2475-2490 (2000).

47. Athanasiou, K. A., Niederauer, G. G. \& Agrawal, C. M. Sterilization, toxicity, biocompatibility and clinical applications of polylactic acid/polyglycolic acid copolymers. Biomaterials 17, 93-102 (1996).

48. Gopferich, A. Mechanisms of polymer degradation and erosion. Biomaterials 17, 103-114 (1996).

49. Park, T. G. Degradation of poly (DL-lactic acid) microspheres: effect of molecular weight. J Control Release 30, 161-173 (1994).

50. Jalil, R. \& Nixon, J. R. Microencapsulation using poly (DL-lactic acid). III: effect of polymer molecular weight on the release kinetics. J Microencapsul 7, 357-374 (1990).

51. Li, S. Hydrolytic degradation characteristics of aliphatic polyesters derived from lactic and glycolic acids. J Biomed Mat Res 48, 342-353 (1999).

52. Ogawa, Y., Yamamoto, M., Takada, S., Okada, H. \& Shimamoto, T. Controlled release of leuprolide acetate from polylactic acid or copoly (lactic/glycolic) acid microcapsules: influence of molecular weight and copolymer ratio of polymer. Chem Pharm Bull 36, 1502-1507 (1988).

53. Omelczuk, M. O. \& McGinity, J. W. The influence of polymer glass transition temperature and molecular weight on drug release from tablets containing poly (DL-lactic acid). Pharm Res 9, 26-32 (1992).

54. Bodmeier, R., Oh, K. H. \& Chen, H. The effect of the addition of low molecular weight poly (DL-lactide) on drug release from biodegradable poly (DL-lactide) drug release systems. Int J Pharm 51, 1-8 (1989). 
55. Cha, Y. \& Pitt, C. G. The acceleration of degradation controlled drug delivery from polyester microspheres. J Control Release 8, (1989).

56. Mauduit, J., Bukh, N. \& Vert, M. Gentamycin/poly (lactic acid) blends aimed at sustained release local antibiotic therapy administered preoperatively. I: the case of gentamycin base and gentamycin sulfate in poly (DL-lactic acid) oligomers. $J$ Control Release 23, 209-220 (1993).

57. Li, S., Girod-Holland, S. \& Vert, M. Hydrolytic degradation of poly ( DL-lactic acid) in the presence of caffeine base. J Control Release 40, 41-53 (1996).

58. Muthu, M. S. Nanoparticles based on PLGA and its co polymer: an overview Asian Journal of Pharmaceutics, 266-273 (2009).

59. Lewis, D. D. Controlled release of bioactive agents from lactide/gycolide polymers. (Marcel Dekker, 1990).

60. Visscher, G. E. et al. Effect of particle size on the in vitro and in vivo degradation rates of poly (DL-lactide-co-glycolide) microcapsules. J Biomed Mat Res 22, 733-746 (1988).

61. Anderson, J. M. In vivo biocompatibility of implantation delivery systems and biomaterials. Eur J Pharm Biopharm 40, 1-8 (1994).

62. Visscher, G. E. et al. Biodegradation of and tissue reaction to poly (DL-lactide) microcapsules. J Biomed Mat Res 20, 667-676 (1986).

63. Hausberger, A. G. \& DeLuca, P. P. Characterization of biodegradable poly (D,Llactide-co-glycolide) polymers and microspheres. J Pharm Biomed Anal 13, 747-760 (1995).

64. Li, S. \& McCarthy, S. P. Influence of crystallinity and stereochemistry on the enzymatic degradation of poly (lactide) Macromolecules 32, 4454-5556 (1999).

65. Jamshidi, K., Hyon, S. H. \& Ikada, Y. Thermal characterization of polylactides. Polymer 29, 2229-2234 (1988).

66. Al Ruhaimi, K. A. Closure of palatal defects without a surgical flap: an experimental study in rabbits. J Oral Maxillofac Surg 59, 1319-1325 (2001).

67. Rothen-Weinhold, A., Besseghir, K., De Zelicourt, Y. \& Gurny, R. Development and evaluation in vivo of a long term delivery system for vapreotide, a somatostatin analogue. J Control Release 52, 205-213 (1998).

68. Negrín, C. M., Delgado, A., Llabrés, M. \& Évora, C. Methadone implants for methadone maintenance treatment: in vitro and in vivo animal studies. J Control Release 95, 413-421 (2004).

69. Rothen-Weinhold, A. et al. Injection molding versus extrusion as manufacturing technique for the preparation of biodegradable implants. Eur J Pharm Biopharm 48, 113-121 (1999).

70. Yamakawa, I., Kawahara, M., Watanabe, S. \& Miyake, Y. Sustained release of insulin by double layered implant using poly (DL-lactic acid). J Pharm Sci 79, 505-509 (1990).

71. Birnbaum, D. T. \& Brannon-Peppas, L. Microparticle drug delivery systems. (Humana Press, 2004).

72. Sinha, V. R. \& Trehan, A. Formulation, characterization, and evaluation of ketorolac tromethamine loaded biodegradable microspheres. Drug Del 12, 133139 (2005). 
73. Smith, A. \& Hunneyball, I. M. Evaluation of poly (lactic acid) as a biodegradable drug delivery system for parenteral administration. Int J Pharm 30, (1986).

74. Chien, Y. W. The use of biocompatible polymers in rate controlled drug delivery systems. Pharm Tech 50-66 (1985).

75. Pandey, S., Singh, U. V. \& Udupa, N. Implantable flurbiprofen for treating inflammation associated with arthritis. Ind Drugs 31, 254-257 (1994).

76. Chu, C. C. Degradation phenonema of two linear aliphatic polyester fiber used in medicine and surgery. Polymerization 26, 591-594 (1985).

77. O'Donnell, P. B., Iwata, M. \& McGinity, J. W. Properties of multiphase microspheres of poly (DL-lactic-co-glycolic acid) prepared by a potentiometric dispersion technique. J Microencapsul 12, 155-163 (1995).

78. O’Donnell, P. B. \& McGinity, J. W. Preparation of microspheres by the solvent evaporation technique. Adv Drug Del Rev 28, 25-42 (1997).

79. Iwata, M. \& McGinity, J. W. Preparation of multi phase microspheres of poly (DL-lactic acid) and poly (DL-lactic-co-glycolic acid) containing a W/O emulsion by a multiple emulsion solvent evaporation technique. J Microencapsul 9, 201-214 (1992).

80. Tabata, Y., Gutta, S. \& Langer, R. Controlled delivery systems for proteins using polyanhydride microspheres. Pharm Res 10, 487-496 (1993).

81. Jalil, R. \& Nixon, J. R. Biodegradable poly (lactic acid) and poly (lactide-coglycolide) microcapsules: problems associated with preparative techniques and release properties. J Microencapsul 7, 297-325 (1990).

82. Nykamp, G., Carstensen, U. \& Muller, B. W. Jet milling-a new technique for microparticle preparation. Int J Pharm 242, 79-86 (2002).

83. Graham, P. D., Brodbeck, K. J. \& McHugh, A. J. Phase inversion dynamics of PLGA solutions related to drug delivery. J Control Release 58, 233-245 (1999).

84. Brodbeck, K. J., DesNoyer, J. R. \& McHugh, A. J. Phase inversion dynamics of PLGA solutions related to drug delivery. Part II: The role of solution thermodynamics and bath side mass transfer. J Control Release 62, 333-344 (1999).

85. Boring, C. C., Squires, T. S. \& Tong, T. Cancer statistics, 1993. CA Cancer J Clin 43, 7-26 (1993).

86. Fujino, M. et al. Some analogs of luteinizing hormone releasing hormone (LHRH) having intense ovulation inducing activity. Biochem Biophys Res Commun 57, 1248-1256 (1974).

87. Kochhar, C. \& Imanidis, G. In vitro transdermal iontophoretic delivery of leuprolide under constant current application. J Control Release 98, 25-35 (2004).

88. Adjei, A., Sundberg, D., Miller, J. \& Chun, A. Bioavailability of leuprolide acetate following nasal and inhalation delivery to rats and healthy humans. Pharm Res 9, 244-249 (1992).

89. Okada, H. et al. Vaginal absorption of a potent luteinizing hormone releasing hormone analog (leuprolide) in rats I: absorption by various routes and absorption enhancement. J Pharm Sci 71, 1367-1371 (1982). 
90. Sharifi, R. \& Soloway, M. Clinical study of leuprolide depot formulation in the treatment of advanced prostate cancer: the leuprolide study group. J Urol 143, 68-71 (1990).

91. Dunn, R. L. \& Tipton, A. J. Polymeric compositions useful as controlled release implants. U.S. patent 5702716 (1997).

92. Gray, V. A. Dissolution testing: an overview and summary of recent trends and regulatory activities. Tablets and Capsules 1, 20-24 (2003).

93. Sirisuth, N. \& Eddington, N. D. In vitro in vivo correlations, systemic methods for the development and validation of an IVIVC metoprolol and naproxen drug examples. Int J Generic Drugs 3, 250-258 (2002).

94. D'Souza, S. S. \& DeLuca, P. P. Development of a dialysis in vitro release method for biodegradable microspheres. AAPS Pharm SciTech 6, 323-328 (2005).

95. Schaefer, M. J. \& Singh, J. Effect of tricaprin on the physical characteristics and in vitro release of etoposide from PLGA microspheres. Biomaterials 23, 34653471 (2002).

96. Ruan, G., Feng, S. S. \& Li, Q. T. Effects of material hydrophobicity on physical properties of polymeric microspheres formed by double emulsion process. $J$ Control Release 84, 151-160 (2002).

97. Ruan, G. \& Feng, S. S. Preparation and characterization of poly (lactic acid)-poly (ethylene glycol)-poly (lactic acid) (PLA-PEG-PLA) microspheres for controlled release of paclitaxel. Biomaterials 24, 5037-5044 (2003).

98. Diwan, M. \& Park, T. G. Stabilization of recombinant interferon alpha by pegylation for encapsulation in PLGA microspheres. Int J Pharm 252, 111-122 (2003).

99. Liu, F. I., Kuo, J. H., Sung, K. C. \& Hu, O. Y. Biodegradable polymeric microspheres for nalbuphine prodrug controlled delivery: in vitro characterization and in vivo pharmacokinetic studies. Int J Pharm 257, 23-31 (2003).

100. Poulain, N. et al. Microspheres based on inulin for the controlled release of serine protease inhibitors: preparation, characterization and in vitro release. J Control Release 92, 27-38 (2003).

101. Iyera, S. S., Barrb, W. H. \& Karnes, H. T. Profiling in vitro drug release from subcutaneous implants: a review of current status and potential implications on drug product development. Biopharm Drug Dispos 27, 157-170 (2006).

102. Faisant, N., Siepmann, J., Richard, J. \& Benoit, J. P. Mathematical modeling of drug release from bioerodible microparticles: effect of gamma irradiation. Eur $J$ Pharm Biopharm 56, 271-279 (2003).

103. Gavini, E. et al. PLGA microspheres for the ocular delivery of a peptide drug, vancomycin using emulsification/spray drying as the preparation method: in vitro/in vivo studies. Eur J Pharm Biopharm 57, 207-212 (2004).

104. Martinez-Sancho, C., Herrero-Vanrell, R. \& Negro, S. Optimisation of aciclovir poly (DL-lactide-co-glycolide) microspheres for intravitreal administration using a factorial design study. Int J Pharm 273, 45-56 (2004).

105. Uhrich, E. K., Cannizzaro, M. S., Langer, S. R. \& Shakessheff, M. K. Polymeric systems for controlled drug release. J Am Chem Soc 99, 3181-3198 (1999). 
106. Jain, R., Shah, N. H., Malick, A. W. \& Rhodes, C. T. Controlled drug delivery by biodegradable poly (ester) devices: different preparative approaches. Drug Dev Ind Pharm 24, 703-727 (1998).

107. Kunou, A. et al. Long term sustained release of ganciclovir from biodegradable scleral implant for the treatment of cytomegalovirus retinitis. $J$ Control Release 68, 263-271 (2000).

108. Jayanthpanayam, Williams, D., Dash, A., Pelecky, D. L. \& Labhasetwar, V. Solid state solubility influences encapsulation and release of hydrophobic drugs from PLGA/PLA nanoparticles. J Pharm Sci 93, (2004).

109. da Silva Juniora, A. A. et al. Thermal behavior and stability of biodegradable spray dried microparticles containing triamcinolone. Int J Pharm 368, 45-55 (2009).

110. Siepmann, F., Le Brun, V. \& Siepmann, J. Drugs acting as plasticizers in polymeric systems: a quantitative treatment. J Control Release 115, 298-306 (2006).

111. Hamishehkara, H. et al. The effect of formulation variables on the characteristics of insulin loaded poly (lactic-co-glycolic acid) microspheres prepared by a single phase oil in oil solvent evaporation method. Colloids and Surfaces B:

Biointerfaces 74, 340-349 (2009).

112. Nemati, M. H. \& Astaneh, B. Optimal management of familial hypercholesterolemia: treatment and management strategies. Vasc Health Risk Manag 6, 1079-1088 (2010).

113. Al-Tahami, K. \& Singh, J. Smart polymer based delivery systems for peptides and proteins. Recent Pat Drug Deliv Formul 1, 65-71 (2007).

114. Ravivarapu, H. B., Moyer, K. L. \& Dunn, R. L. Sustained activity and release of leuprolide acetate from an in situ forming polymeric implant. AAPS Pharm Sci Tech 1, 1 (2000).

115. Sales-Junior, P. A. et al. Use of biodegradable PLGA microspheres as a slow release delivery system for the boophilus microplus synthetic vaccine SBm7462. Veterinary Immunology and Immunopathology 107, 281-290 (2005).

116. Luan, X. \& Bodmeier, R. Modification of the triphasic drug release pattern of leuprolide acetate loaded poly (lactide-co-glycolide) microparticles. Eur J Pharm Biopharm 63, 205-214 (2006).

117. Loo, S. C., Tan, Z. Y., Chow, Y. J. \& Lin, S. L. Drug release from irradiated PLGA and PLLA multi layered films. J Pharm Sci 99, 3060-3071 (2010).

118. Luan, X. \& Bodmeier, R. In situ forming microparticle system for controlled delivery of leuprolide acetate: influence of the formulation and processing parameters. Eur J Pharm Sci 27, 143-149 (2006).

119. Joachim Loo, S. C. et al. Hydrolytic degradation characteristics of irradiated multi layered PLGA films. Int J Pharm 360, 228-230 (2008).

120. Meng, B. et al. Effect of medium chain triglycerides on the release behavior of endostar encapsulated PLGA microspheres. Int J Pharm 397, 136-143 (2010).

121. Kwon, Y. M. \& Kim, S. W. Biodegradable triblock copolymer microspheres based on thermosensitive sol gel transition. Pharm Res 21, 339-343 (2004). 
122. Lara, M. G., Bentley, M. V. \& Collett, J. H. In vitro drug release mechanism and drug loading studies of cubic phase gels. Int J Pharm 293, 241-250 (2005).

123. Ahmed, A. R., Dashevsky, A. \& Bodmeier, R. Reduction in burst release of PLGA microparticles by incorporation into cubic phase forming systems. Eur $J$ Pharm Biopharm 70, 765-769 (2008).

124. Wang, X., Venkatraman, S. S., Boey, F. Y., Loo, J. S. \& Tan, L. P. Controlled release of sirolimus from a multilayered PLGA stent matrix. Biomaterials 27, 5588-5595 (2006).

125. Fogueri, L. R. \& Singh, S. Smart polymers for controlled delivery of proteins and peptides: a review of patents. Recent Pat Drug Del Form 3, 40-48 (2009).

126. Luan, X. \& Bodmeier, R. Influence of the poly (lactide-co-glycolide) type on the leuprolide release from in situ forming microparticle systems. J Control Release 110, 266-272 (2006).

127. Leo, E., Scatturin, A., Vighi, E. \& Dalpiaz, A. Polymeric nanoparticles as drug controlled release systems: a new formulation strategy for drugs with small or large molecular weight. J Nanosci Nanotechnol 6, 3070-3079 (2006).

128. Schenderlein, S., Luck, M. \& Muller, B. W. Partial solubility parameters of poly (DL-lactide-co-glycolide). Int J Pharm 286, 19-26 (2004).

129. Yaghmur, A., de Campo, L., Sagalowicz, L., Leser, M. E. \& Glatter, O. Emulsified microemulsions and oil containing liquid crystalline phases. Langmuir 21, 569-577 (2005).

130. Yaghmur, A. et al. Oil loaded monolinolein based particles with confined inverse discontinuous cubic structure (Fd3m). Langmuir 22, 517-521 (2006).

131. Vert, M., Li, S. \& Garreau, H. New insights on the degradation of bioresorbable polymeric devices based on lactic and glycolic acids. Clin Mater 10, 3-8 (1992).

132. Detrembleur, C. et al. $\gamma$-bromo- $\varepsilon$-caprolactone, a versatile lactone for the synthesis of new functional aliphatic polyesters Macromolecules 33, 7751 (2000).

133. Sah, H. \& Chien, Y. W. Degradability and antigen release characteristics of polyester microspheres prepared from polymer blends. J Appl Polym Sci 197 (1995).

134. Sah, H., Toddywala, R. \& Chien, Y. W. The influence of microcapsule formulations on the controlled release of a protein. $J$ Control Release 30, 201 (1994).

135. Sah, H., Toddywala, R. \& Chien, Y. W. Continuous release of proteins from biodegradable microcapsules and in vivo evaluation of their potential as a vaccine adjuvant. J Control Release 35, 137 (1995).

136. Pitt, C. G. Poly ( $\varepsilon$-caprolactone) and its co polymers. (Marcel Decker, 1990).

137. Perez, M. H. et al. The preparation and evaluation of poly ( $\varepsilon$-caprolactone) microsparticles containing both lipophillic and hydrophilic drug. $J$ Control Release 65, 429-438 (2000).

138. Dordunoo, S. K. et al. Taxol encapsulation in poly (epsilon-caprolactone) microspheres. Cancer Chemother Pharmacol 36, 279-282 (1995).

139. Sondhof, A. F. et al. Polylactic acid (biodegradable) microspheres for sustained antimicrobial drug release in the equine joint: preliminary in vivo results. Int $J$ Symp Control Rel Bioact Mat 25, (1998). 
140. Das, G. S., Rao, G. H., Wilson, R. F. \& Chandy, T. Colchicine encapsulation within poly (ethylene glycol) coated poly (lactic acid)/poly (epsiloncaprolactone) microspheres controlled release studies. Drug Del 7, 129-138 (2000).

141. Chang, R. K., Price, J. C. \& Whitworth, C. W. Control of drug release rate by use of mixtures of poly ( $\varepsilon$-caprolactone) and cellulose acetate butyrate polymers. Drug Dev Ind Pharm 13, 1119-1135 (1987).

142. Aberturas, M. R., Molpeceres, J., Guzman, M. \& Garcia, F. Development of a new cyclosporine formulation based on poly (caprolactone) microspheres. $J$ Microencapsul 19, 61-72 (2002).

143. Chandy, T., Wilson, R. F., Rao, G. H. \& Das, G. S. Changes in cisplatin delivery due to surface coated poly (lactic acid) poly (epsilon-caprolactone) microspheres. J Biomater Appl 16, 275-291 (2002).

144. Ming, C. \& Chan, L. L. Direct observation of the growth of lamellae and spherulites by AFM. Adv Polym Sci (2005).

145. Bassett, D. C. Principles of polymer morphology. (Cambridge University Press, 1981).

146. Woodward, A. E. Atlas of polymer morphology. (Hanser Publisher, 1989).

147. Beekmans, L. G. M. \& Vancso, G. J. Real time crystallization study of poly (ecaprolactone) by hot stage atomic force microscopy. Polymer 41, 8975 (2000).

148. Akhtar, S., Pouton, C. W. \& Notarianni, L. J. Crystallization behaviour and drug release from bacterial polyhydroxyalkanoates. Polymer 33, 117-126 (1992).

149. Yang, A., Wu, R. \& Zhu, P. Thermal analysis and miscibility of chitin/polycaprolactone blends. J Appl Polym Sci 81, 3117-3123 (2001).

150. Enevoldsen, L. H., Simonsen, L., Stallknecht, B., Galbo, H. \& Bulow, J. In vivo human lipolytic activity in preperitoneal and subdivisions of subcutaneous abdominal adipose tissue. Am J Physiol Endocrinol Metab 281, 1110-1114 (2001).

151. Boni, J. E., Brickl, R. S., Dressman, J. \& Pfefferle, M. L. Instant FaSSIF and FeSSIF-biorelevance meets practicality. Dissolution Technologies, 41-45 (2009).

152. Kipshidze, N. N., Tsapenko, M. V., Leon, M. B., Stone, G. W. \& Moses, J. W. Update on drug eluting coronary stents. Expert Rev Cardiovasc Ther 3, 953-968 (2005).

153. Moses, J. W., Kipshidze, N. \& Leon, M. B. Perspectives of drug eluting stents: the next revolution. Am J Cardiovasc Drugs 2, 163-172 (2002).

154. Klonoff, D. C. Technological advances in the treatment of diabetes mellitus: better bioengineering begets benefits in glucose measurement, the artificial pancreas, and insulin delivery. Pediatr Endocrinol Rev 1, 94-100 (2003).

155. Frazier, O. H. \& Jacob, L. P. Small pumps for ventricular assistance: progress in mechanical circulatory support. Cardiol Clin 25, 553-564 (2007).

156. Belverud, S., Mogilner, A. \& Schulder, M. Intrathecal pumps. Neurotherapeutics 5, 114-122 (2008).

157. Kizilel, S., Garfinkel, M. \& Opara, E. The bioartificial pancreas: progress and challenges. Diabetes Technol Ther 7, 968-985 (2005). 
158. O'Connor, R. C., Lyon, M. B., Guralnick, M. L. \& Bales, G. T. Long term follow up of single versus double cuff artificial urinary sphincter insertion for the treatment of severe postprostatectomy stress urinary incontinence. Urology 71, 90-93 (2008).

159. Fine, D. M. \& Tobias, A. H. Cardiovascular device infections in dogs: report of 8 cases and review of the literature. J Vet Intern Med 21, 1265-1271 (2007).

160. Prokop, A. Bioartificial organs in the twenty first century: nanobiological devices. Ann N Y Acad Sci 944, 472-490 (2001).

161. Wilson, G. S. et al. Progress toward the development of an implantable sensor for glucose. Clin Chem 38, 1613-1617 (1992).

162. Koschwanez, H. E. \& Reichert, W. M. In vitro, in vivo and post explantation testing of glucose detecting biosensors: current methods and recommendations.

Biomaterials 28, 3687-3703 (2007).

163. Callahan, T. D. t. \& Natale, A. Catheter ablation of atrial fibrillation. Med Clin North Am 92, 179-201 (2008).

164. Matsumoto, T. et al. Modification of apatite materials for bone tissue engineering and drug delivery carriers. Curr Med Chem 14, 2726-2733 (2007).

165. Salgado, A. J., Coutinho, O. P. \& Reis, R. L. Bone tissue engineering: state of the art and future trends. Macromol Biosci 4, 743-765 (2004).

166. Abukawa, H. et al. The engineering of craniofacial tissues in the laboratory: a review of biomaterials for scaffolds and implant coatings. Dent Clin North Am 50, 205-216 (2006).

167. Nozynski, J. K., Religa, Z., Wszolek, J., Zembala-Nozynska, E. \& Rozentryt, P. Biological heart valve-an alternative to mechanical valve. Med Sci Monit 7, 550562 (2001).

168. Black, M. M. \& Drury, P. J. Mechanical and other problems of artificial valves. Curr Top Pathol 86, 127-159 (1994).

169. Uchegbu, I. F. et al. Polymeric chitosan based vesicles for drug delivery. J Pharm Pharmacol 50, 453-458 (1998).

170. Khor, E. \& Lim, L. Y. Implantable applications of chitin and chitosan. Biomaterials 24, 2339-2349 (2003).

171. Borchard, G. \& Junginger, H. E. Modern drug delivery applications of chitosan. Adv Drug Del Rev 52, 103 (2001).

172. de Vos, P., Hoogmoed, C. G. \& Busscher, H. J. Chemistry and biocompatibility of alginate PLL capsules for immunoprotection of mammalian cells. J Biomed Mat Res 60, 252-259 (2002).

173. Sano, A., Hojo, T., Maeda, M. \& Fujioka, K. Protein release from collagen matrices. Adv Drug Del Rev 31, 247-266 (1998).

174. Geiger, M., Li, R. H. \& Friess, W. Collagen sponges for bone regeneration with rhBMP-2. Adv Drug Del Rev 55, 1613-1629 (2003).

175. Vercruysse, K. P. \& Prestwich, G. D. Hyaluronate derivatives in drug delivery. Crit Rev Ther Drug Carrier Syst 15, 513-555 (1998). 
176. Cadee, J. A., Brouwer, L. A., den Otter, W., Hennink, W. E. \& van Luyn, M. J. A comparative biocompatibility study of microspheres based on crosslinked dextran or poly (lactic-co-glycolic) acid after subcutaneous injection in rats. J Biomed Mat Res 56, 600-609 (2001).

177. Draye, J. P. et al. In vitro and in vivo biocompatibility of dextran dialdehyde cross linked gelatin hydrogel films. Biomaterials 19, 1677-1687 (1998).

178. Daugherty, A. L., Cleland, J. L., Duenas, E. M. \& Mrsny, R. J. Pharmacological modulation of the tissue response to implanted polylactic-co-glycolic acid microspheres. Eur J Pharm Biopharm 44, 89-102 (1997).

179. Lunsford, L., McKeever, U., Eckstein, V. \& Hedley, M. L. Tissue distribution and persistence in mice of plasmid DNA encapsulated in a PLGA based microsphere delivery vehicle. J Drug Target 8, 39-50 (2000).

180. Ronneberger, B., Kissel, T. \& Anderson, J. M. Biocompatibility of ABA triblock copolymer microparticles consisting of poly (L-lactic-co-glycolic-acid) A blocks attached to central poly (oxyethylene) B blocks in rats after intramuscular injection. Eur J Pharm Biopharm 43, 19-28 (1997).

181. Ronneberger, B., Kao, W. J., Anderson, J. M. \& Kissel, T. In vivo biocompatibility study of ABA triblock copolymers consisting of poly (L-lacticco-glycolic acid) A blocks attached to central poly (oxyethylene) B blocks. $J$ Biomed Mat Res 30, 31-40 (1996).

182. Royals, M. A. et al. Biocompatibility of a biodegradable in situ forming implant system in rhesus monkeys. J Biomed Mat Res 45, 231-239 (1999).

183. Dalsin, J. L., Hu, B. H., Lee, B. P. \& Messersmith, P. B. Mussel adhesive protein mimetic polymers for the preparation of nonfouling surfaces. J Am Chem Soc 125, 4253-4258 (2003).

184. Shen, M. \& Horbett, T. A. The effects of surface chemistry and adsorbed proteins on monocyte/macrophage adhesion to chemically modified polystyrene surfaces. J Biomed Mat Res 57, 336-345 (2001).

185. Paradossi, G., Cavalieri, F., Chiessi, E., Spagnoli, C. \& Cowman, M. K. Poly (vinyl alcohol) as versatile biomaterial for potential biomedical applications. $J$ Mat Sci Mat Med 14, 687-691 (2003).

186. Oka, M. et al. Development of artificial articular cartilage. Proc Inst Mech Eng $H$ 214, 59-68 (2000).

187. Maruoka, S. et al. Biocompatibility of polyvinylalcohol gel as a vitreous substitute. Curr Eye Res 31, 599-606 (2006).

188. Anderson, J. M. Biological responses to materials. Annu Rev Mater Res 31, 81110 (2001).

189. Anderson, J. M. Mechanisms of inflammation and infection with implanted devices. Cardiovasc Pathol 2, 33-41 (1993).

190. Anderson, J. M. Inflammatory response to implants. ASAIO Trans 34, 101-107 (1988).

191. Cotran, R. Z., Kumar, V. \& Robbins, S. L. Inflammation and repair. (WB Saunders, 1999).

192. Gallin, J. I. \& Synderman, R. Inflammation: basic principles and clinical correlates. (Raven, 1999). 
193. Clark, R. A. et al. Fibronectin and fibrin provide a provisional matrix for epidermal cell migration during wound reepithelialization. J Invest Dermatol 79, 264-269 (1982).

194. Tang, L. \& Eaton, J. W. Fibrinogen mediates acute inflammatory responses to biomaterials. $J$ Exp Med 178, 2147-2156 (1993).

195. Tang, L. Mechanisms of fibrinogen domains: biomaterial interactions. J Biomat Sci Polym Ed 9, 1257-1266 (1998).

196. Broadley, K. N. et al. Monospecific antibodies implicate basic fibroblast growth factor in normal wound repair. Lab Invest 61, 571-575 (1989).

197. Sporn, M. B. \& Roberts, A. B. Peptide growth factors are multifunctional. Nature 332, 217-219 (1988).

198. Muller, G., Behrens, J., Nussbaumer, U., Bohlen, P. \& Birchmeier, W. Inhibitory action of transforming growth factor beta on endothelial cells. Proc Natl Acad Sci $U S A$ 84, 5600-5604 (1987).

199. Madri, J. A., Pratt, B. M. \& Tucker, A. M. Phenotypic modulation of endothelial cells by transforming growth factor beta depends upon the composition and organization of the extracellular matrix. J Cell Biol 106, 1375-1384 (1988).

200. Wahl, S. M. et al. Transforming growth factor type beta induces monocyte chemotaxis and growth factor production. Proc Natl Acad Sci U S A 84, 57885792 (1987).

201. Ignotz, R. A., Endo, T. \& Massague, J. Regulation of fibronectin and type I collagen mRNA levels by transforming growth factor beta. J Biol Chem 262, 6443-6446 (1987).

202. Spector, M., Cease, C. \& Tong-Li, X. The local tissue response to biomaterial. Crit Rev Biocompatibility 5, 269-295 (1989).

203. Wokalek, H. \& Ruh, H. Time course of wound healing. J Biomat Appl 5, 337-362 (1991).

204. Ganz, T. Neutrophil receptors. Ann Intern Med 109, 127-142 (1988).

205. Henson, P. M. \& Johnston, R. B., Jr. Tissue injury in inflammation: oxidants, proteinases, and cationic proteins. J Clin Invest 79, 669-674 (1987).

206. Malech, H. L. \& Gallin, J. I. Neutrophils in human diseases. N Engl J Med 317, 687-694 (1987).

207. Johnston, R. B. Current concepts: immunology, monocytes and macrophages. $N$ Engl J Med 318, 747-752 (1988).

208. Williams, G. T. \& Williams, W. J. Granulomatous inflammation-a review. J Clin Pathol 36, 723-733 (1983).

209. Yamaguchi, K. \& Anderson, J. M. In vivo biocompatibility studies of medisorb 65/35 dl-lactide/glycolide copolymer microspheres. J Control Release 24, 81-93 (1993).

210. Serrano, M. C. et al. In vitro biocompatibility assessment of poly (epsiloncaprolactone) films using L929 mouse fibroblasts. Biomaterials 25, 5603-5611 (2004).

211. Al Ruhaimi, K. A. Bone graft substitutes: a comparative qualitative histologic review of current osteoconductive grafting materials. Int J Oral Maxillofac Implants 16, 105-114 (2001). 
212. Pollick, S., Shors, E. C., Holmes, R. E. \& Kraut, R. A. Bone formation and implant degradation of coralline porous ceramics placed in bone and ectopic sites. J Oral Maxillofac Surg 53, 915-922 (1995).

213. da Cruz, A. C. et al. Physicochemical characterization and biocompatibility evaluation of hydroxyapatites. J Oral Sci 48, 219-226 (2006). 


\section{VITA}

Ms. Sonia Bedi, daughter of Mrs. Ravinder Kaur and Mr. Rajinder Singh Bedi, was born in Delhi, India in 1980. She graduated with a Bachelor of Pharmacy degree from Delhi College of Pharmacy, India in June 2002. She completed her Masters in Pharmacy (M.Pharm.) from National Institute of Pharmaceutical Education and Research (NIPER) in December 2003 following which she joined Ranbaxy Research Labs where she worked as a Research Associate in Intellectual Property for 2 years. Later she joined the graduate school at The University of Tennessee Health Science Center in the Fall of 2006 where she was awarded a Graduate Teaching Assistantship. She has a GATE (Graduate Aptitude Test for Engineers) score of 99.93 percentile and an All India Rank 3 (2002). She is a member of the Rho Chi Pharmaceutical Honorary Society, American Association of Pharmaceutical Scientists (AAPS), Parenteral Drug Association (PDA) and Controlled Release Society (CRS). Ms. Bedi has presented her work on several projects at the international conferences and filed a provisional patent application with USPTO for one of her projects with the help of significant contribution from UTRF. 